\title{
Employing laboratory physical modeling to study the radio imaging method (RIM)
}

Jun Lu

West Virginia University

Follow this and additional works at: https://researchrepository.wvu.edu/etd

\section{Recommended Citation}

Lu, Jun, "Employing laboratory physical modeling to study the radio imaging method (RIM)" (2009). Graduate Theses, Dissertations, and Problem Reports. 2883.

https://researchrepository.wvu.edu/etd/2883

This Dissertation is protected by copyright and/or related rights. It has been brought to you by the The Research Repository @ WVU with permission from the rights-holder(s). You are free to use this Dissertation in any way that is permitted by the copyright and related rights legislation that applies to your use. For other uses you must obtain permission from the rights-holder(s) directly, unless additional rights are indicated by a Creative Commons license in the record and/ or on the work itself. This Dissertation has been accepted for inclusion in WVU Graduate Theses, Dissertations, and Problem Reports collection by an authorized administrator of The Research Repository @ WVU.

For more information, please contact researchrepository@mail.wvu.edu. 


\title{
EMPLOYING LABORATORY PHYSICAL MODELING TO STUDY THE RADIO IMAGING METHOD (RIM)
}

\author{
Jun $\mathrm{Lu}$ \\ Dissertation Submitted to the \\ College of Engineering and Mineral Resource \\ at West Virginia University \\ in partial fulfillment of the requirements \\ for the degree of \\ Doctor of Philosophy \\ In \\ Mining Engineering \\ Syd S. Peng, Ph.D. Chair \\ Keith A. Heasley, Ph.D. \\ Thomas H. Wilson, Ph.D. \\ Yi Luo, Ph.D. \\ Felicia Peng, Ph.D. \\ Department of Mining Engineering \\ Morgantown, West Virginia \\ 2009
}

Keywords: Radio Imaging Method (RIM), Geological Anomaly, Electromagnetic Wave, Attenuation Rate, Physical Model, Longwall Mining

Copyright 2009 Jun Lu 


\section{ABSTRACT \\ Employing Laboratory Physical Modeling to Study the Radio Imaging Method (RIM)}

\section{Jun Lu}

Geological anomalies such as faults, sandstone intrusions, sudden thinning and severe undulation of coal seam will greatly affect the production and safety of mining operations. Since most of these geological anomalies in coal mines can increase the attenuation of the electromagnetic signal, the radio imaging method (RIM) is capable of locating the zones of geologic anomalies in underground mines. By using a low frequency EM signal, the RIM technology is the most promising geophysical tool for exploring the geological anomalies ahead of the modern longwall faces which are normally wider than $1,000 \mathrm{ft}$. However, when a number of anomalies co-exist in an area (very common), it becomes difficult for the RIM technology to differentiate the contributions of each individual anomalous factor. This will affect the interpretation accuracy of RIM technology.

In order to increase the accuracy of RIM technology, physical modeling has been employed to investigate the capabilities and limitations of RIM technology. In the scale models, the coal seam, the floor and roof strata and the geological anomalies are simulated with properly chosen simulation materials. Based on the physical modeling simulation theory and laboratory test, different ratios of sand, gypsum, and cement are used to build the floor and roof strata in the scale models. A mixture of sand, coal powder, gypsum was used to simulate the coal seam. Due to the small size of the physical models, a highfrequency EM signal has to be used in the model testing to ensure the "similarity" between the actual longwall panels and the scale models. In a modified radar instrumentation setup, two $900 \mathrm{MHz}$ ground penetrating radar (GPR) antennas are used as the RIM transmitter and receiver, respectively. RIM surveys were then conducted on the scale models. The testing data were used to generate attenuation rate tomograms which in turn were compared with the embedded geological anomalies.

In this study, the theory of applying EM method on scale physical modeling is presented. The designs and constructions of the scale models to represent different geological anomalies are described. The tomogram software is developed and validated. The testing procedure and results are also shown. The generated tomograms are analyzed against the known geological anomalies. 


\section{ACKNOWLEDGMENT}

I would like to express my deepest appreciation to my committee chair, Dr. Syd S. Peng for his invaluable instructions, encouragement, and support during this research and throughout my graduate study. Without his guidance and persistent help this dissertation would not have been possible.

Special thanks are given to the committee members, Dr. Yi Luo, Dr. Keith A. Heasley, Dr. Thomas H. Wilson, and Dr. Felicia Peng for the time and the expertise contributed to the enhancement of this research.

Special thanks are also given to my friends William D. Monaghan from NIOSH and Xiaochao Tang for their great assistances during this research.

Finally, Special thanks are given to my beloved wife, Lihong Zhou, my son Larry and my daughter Nina. Sincere gratitude goes to my family members, my father and mother, my sisters. Without their endless love, constant support, patience, understanding and generous support, I would never have reach this point. 


\section{TABLE OF CONTENTS}

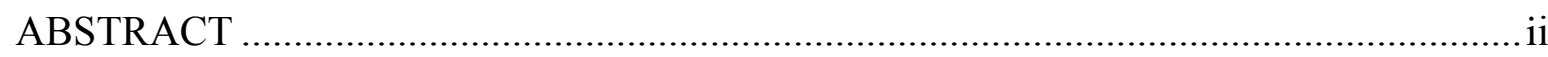

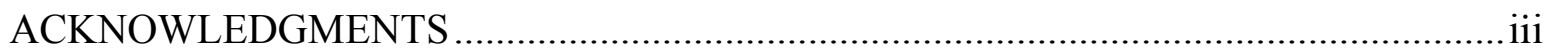

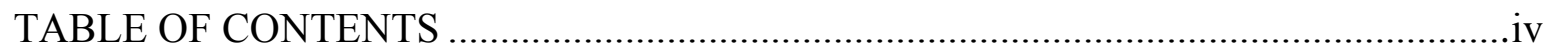

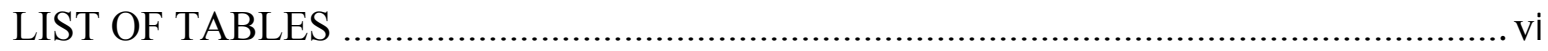

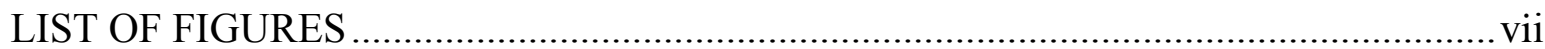

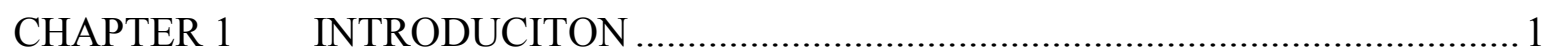

1.1 Geological Anomalies in Longwall Mining ............................................ 1

1.2 Radio Imaging Method (RIM) .......................................................... 2

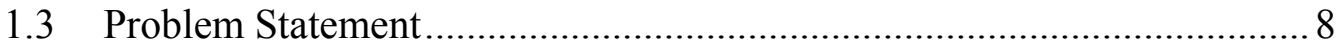

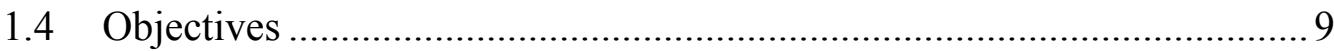

1.5 Scope

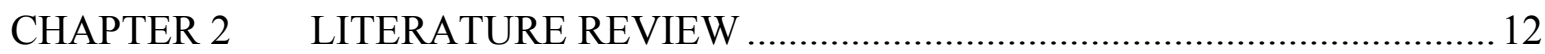

2.1 Electrical Properties of Rock/Coal ........................................................ 12

2.1.1 Dielectric Constant (DC) /Permittivity ........................................ 12

2.1.2 Electrical Conductivity (EC) /Electrical Resistivity (ER) ........... 17

2.1.3 Magnetic Permeability ………………………………………...20

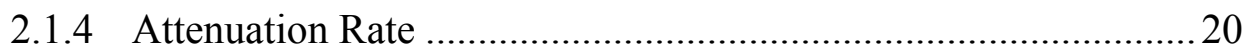

2.2 RIM and Electromagnetic (EM) Seam Wave ………………….............25

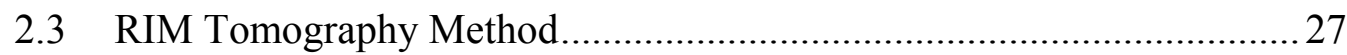

2.4 EM Scale Model Research Method ..........................................................35

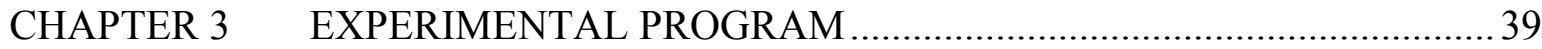

3.1 EM Properties Testing of Simulation Materials ........................................39

3.2 Laboratory Physical Model Testing Equipment ........................................ 46

3.3 Physical Model Testing Procedure ........................................................ 48

3.3.1 Calibration Testing Method ....................................................... 48

3.3.2 Reconnaissance Testing Method...................................................50

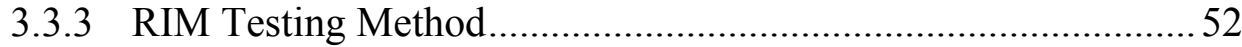

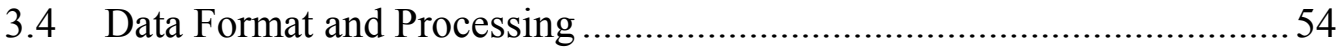

3.5 RIM Tomogram Software Development and Validation ..........................55 
3.5.1 Solving for Attenuation Rates of the Laboratory Physical Model... 55

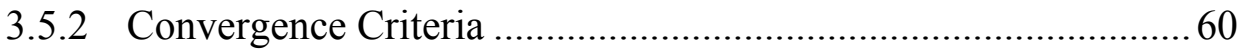

3.5.3 Forward Modeling ........................................................................ 62

CHAPTER 4 PHYSICAL MODELS TESTING AND DATA ANALYSIS .................76

4.1 First Scale Model Study....................................................................

$4.2 \quad$ Second Scale Model Study ……………………….................................. 79

4.2.1 Scale Factors Determination ....................................................... 79

4.2.2 Selection of Physical Model Materials ........................................ 82

4.2.3 Model Design and Construction .................................................. 84

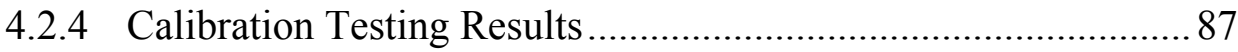

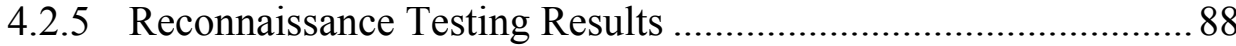

4.2.6 RIM Testing Results ............................................................... 91

CHAPTER 5 SUMMARY AND CONCLUSIONS ………………………………..... 105

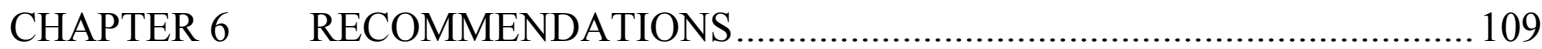

REFERENCES

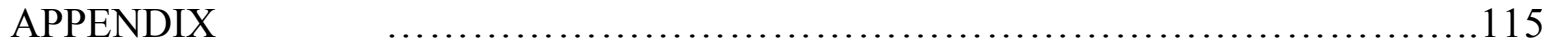




\section{LIST OF TABLES}

Table 2.1 Bulk dielectric constants (measured at $100 \mathrm{MHz}$ ) of common earth materials ... 15

Table 2.2 The electrical resistivity for some typical earth materials (Johnson, 2005) ........ 18

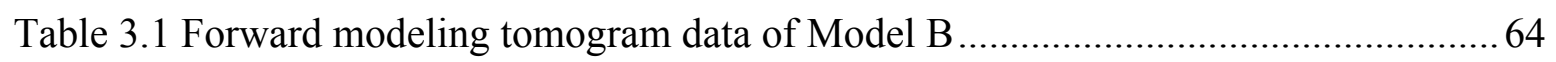

Table 3.2 Relative error of forward modeling tomogram data of Model B ....................... 64

Table 3.3 Forward modeling tomogram data of Model E .............................................6

Table 3.4 Relative error of forward modeling tomogram data of Model E ...................... 67

Table 3.5 Forward modeling tomogram data of Model C...............................................69

Table 3.6 Relative error of forward modeling tomogram data of Model C ......................69

Table 3.7 Forward modeling tomogram data of Model D............................................... 71

Table 3.8 Relative error of forward modeling tomogram data of Model D ..................... 72

Table 3.9 Forward modeling tomogram data of Model D............................................. 74

Table 3.10 Relative error of forward modeling tomogram data of Model D ......................74

Table 4.1 EM properties used in full-sized system and scale physical model ................... 82 


\section{LIST OF FIGURES}

Figure 1.1 Geological anomalies in front of Longwall panel (Stolarczyk and Fry, 1990)....2

Figure 1.2 Layout of RIM working condition and instrumentation (Stolar Horizon, Inc,

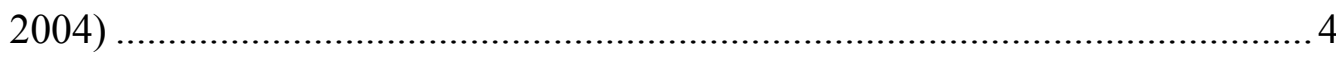

Figure 1.3 RIM testing procedure (Stolarczyk et al., 2006) .............................................. 5

Figure 1.4 Ray path plot and tomogram at a longwall panel with $290-\mathrm{kHz}$ RIM test (Stolarczyk et al., 2006) ................................................................................... 6

Figure 1.5 RIM-IV Hardware (Stolarczyk and Peng, 2003) .............................................. 7

Figure 1.6 Laboratory RIM study methodology ............................................................ 10

Figure 2.1 Dielectric constant measurement equipment (network analyzer and probe kit). 14

Figure 2.2 Four-dimensional representation of the TP-model input parameters (matrix mineralogy, porosity, and water saturation) and resulting bulk dielectric

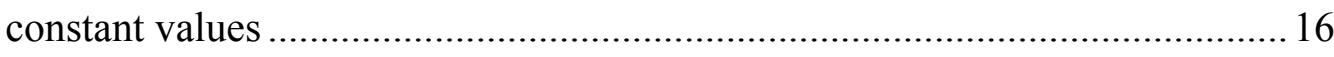

Figure 2.3 Comparisons of the dielectric constants at different moisture levels for a coal sample from the Sewickley Seam (Boykov, 2006) ........................................... 17

Figure 2.4 The effect of water, carbon and salt in soil on resistivity (Anggoro et al., 2006)

Figure 2.5 Comparisons of the electrical resistivity observed at different moisture levels for a coal sample from the Sewickley Seam (Boykov, 2006) ................................. 19

Figure 2.6 Attenuation rate (dB/ft) versus frequency exhibited by: a) shale/clay, b) coal with high moisture, c) range for US coals (Stolarczyk and Peng, 2003) .......... 22

Figure 2.7 Coal seam EM wave attenuation rate versus boundary rock conductivity (Stolarczyk and Peng, 2003) .......................................................................22

Figure 2.8 Sensitivity of radio waves to changes in coal layer thickness (Stolarczyk and Fry, 1990) 24

Figure 2.9 Energy flow along the ray path from the ransmitting (TX) to Receiving (RX) antenna (Stolarczyk and Fry, 1990) .............................................................. 24

Figure 2.10 Attenuation rate/phase shift vs coal conductivity $(H i l l, 1984)$.........................25 
Figure 2.11 Natural waveguide for electromagnetic wave transmission (Stolar Horizon, Inc, 2004) 26

Figure 2.12 Radio wave signal paths in a coal seam (Stolarczyk and Fry, 1990)..............28

Figure 2.13 Ray path and cell index of RIM test....................................................... 29

Figure 2.14 Geometry for the $\frac{i^{t h}}{\text { ray. }} \underline{\alpha_{j}}$ is the attenuation rate in the $j^{\text {th }}$ cell, and rij is the length of the ith ray through the jth cell................................................... 30

Figure 2.15 Method of projection (Zhao, 2005)..................................................... 34

Figure 2.16 Laboratory scale GPR system. (a) Real system and (b) configuration .............38

Figure 3.1 Part of the samples with different ratios of sand, gypsum, cement, salt, and water 40

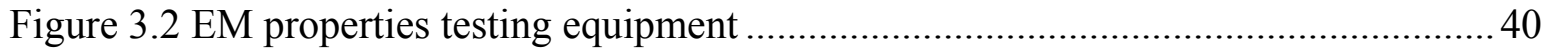

Figure 3.3 Water content effect on dielectric constant and electrical conductivity $(\mathrm{S}: \mathrm{G}: \mathrm{C}: \mathrm{SA}=7: 0.5: 0.5: 1)$. 42

Figure 3.4 Water content and salt content effect on dielectric constant and electrical conductivity.

Figure 3.5 Dielectric constant at different components mixture ratios and different water content to simulate the roof/floor.

Figure 3.6 Electrical conductivity at different components mixture ratios and different water content to simulate the roof/floor. 44

Figure 3.7 Dielectric constant at different components mixture ratios and different water content to simulate the coal seam

Figure 3.8 Electrical conductivity at different components mixture ratios and different water content to simulate the coal seam..................................................... 46

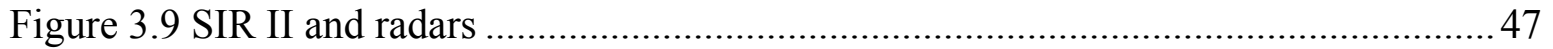

Figure 3.10 Scale model testing system layout ..................................................... 47

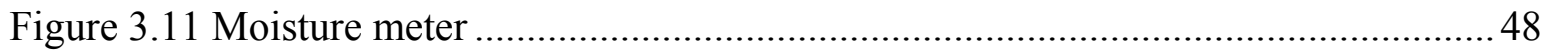

Figure 3.12 102.5-kHz calibration testing results at a longwall panel (Stolar Horizon, Inc.,

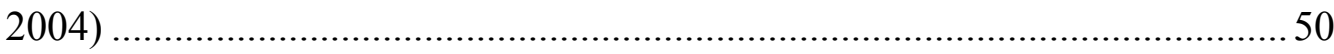

Figure 3.13 Layout of reconnaissance testing .......................................................... 51

Figure 3.14 Reconnaissance testing results at a narrow panel intersected by dikes

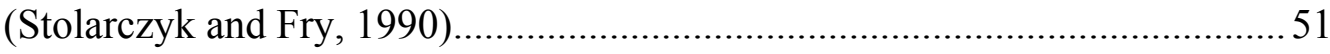


Figure 3.15 Reconnaissance testing results (Stolarczyk et al., 2006)...............................53

Figure 3.16 RIM testing results (Stolarczyk et al., 2006) .............................................53

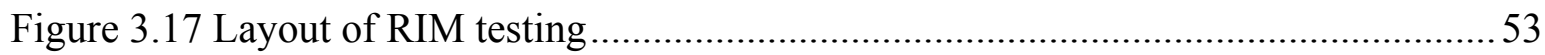

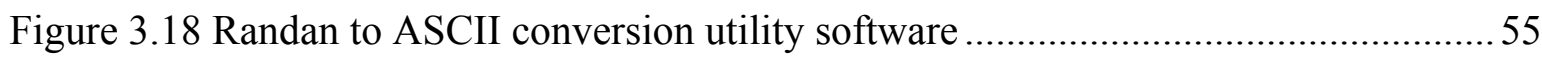

Figure 3.19 Typical Converted EM Waveform (Monaghan, 2007) ................................. 55

Figure 3.20 Interface of RIM tomogram software ..................................................... 59

Figure 3.21 An example of a SIRT algorithm converging to an asymptotic average error-

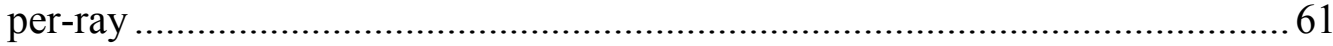

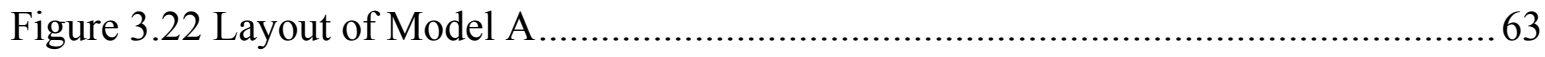

Figure 3.23 Forward modeling tomogram of Model A ................................................63

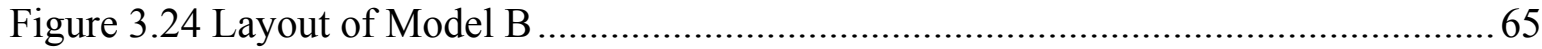

Figure 3.25 Forward modeling tomogram of Model B ..............................................66

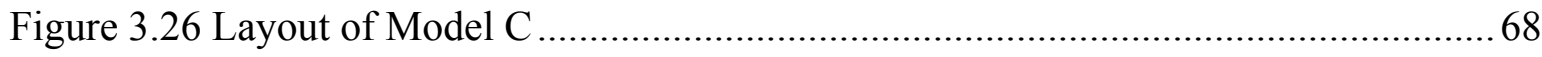

Figure 3.27 Forward modeling tomogram of Model C ............................................... 68

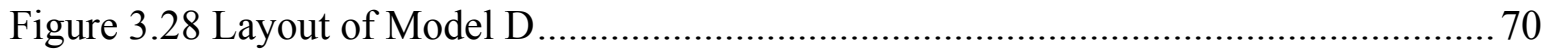

Figure 3.29 Forward modeling tomogram of Model D ............................................... 71

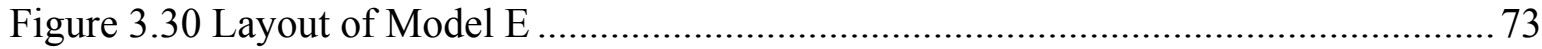

Figure 3.31 Forward modeling tomogram of Model D ............................................... 73

Figure 4.1 Wood box used to build the scale physical model ........................................ 76

Figure 4.2 Layout of the first scale model............................................................... 77

Figure 4.3 Reconnaissance test results for the coal seam and the floor .......................... 78

Figure 4.4 The layout of the physical model ............................................................. 81

Figure 4.5 Materials with mixture ratio of $\mathrm{S}: \mathrm{G}: \mathrm{C}: \mathrm{SA}=7: 0.5: 0.5: 1$ with water content at $6.5 \%$ meet the requirement to simulate roof/floor ..................................... 83

Figure 4.6 Materials with mixture ratio of $\mathrm{S}: \mathrm{Co}: \mathrm{C}=5: 5: 1$ with water content at $6 \%$ meet the requirement to simulate coal seam..................................................... 84

Figure 4.7 Layout of the geological anomalies in the coal seam .................................. 86

Figure 4.8 Layout of the second scale model ......................................................... 86

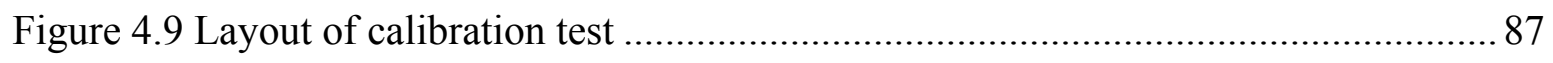

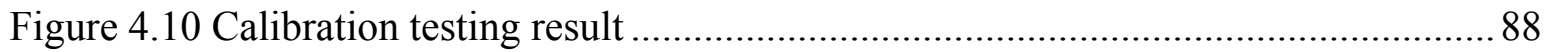

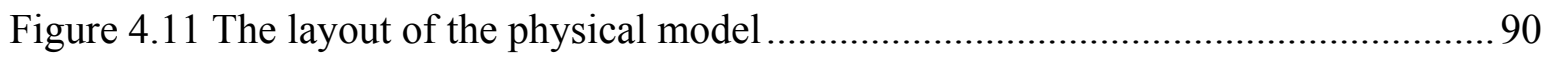


Figure 4.12 Signal strength at the three reconnaissance testing lines of coal seam, roof and floor 90

Figure 4.13 Attenuation rate at the three reconnaissance testing lines of coal seam, roof and floor 91

Figure 4.14 Contour of the attenuation rate along the coal seam testing line ................... 91

Figure 4.15 RIM testing along the coal seam.......................................................... 92

Figure 4.16 Physical model RIM testing scheme and ray paths..................................... 93

Figure 4.17 Signal strength at different receiver stations (transmitter station No.8) ..........95

Figure 4.18 Divided cells and the index of cells for the physical model ......................... 97

Figure 4.19 Layout of forward model which has the same size and anomaly structures as

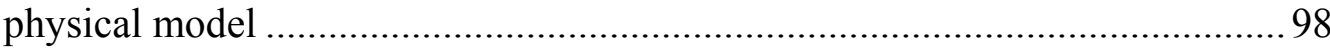

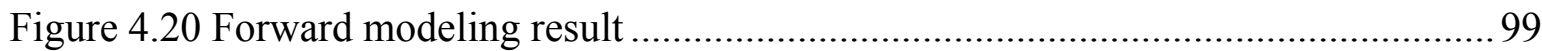

Figure 4.21 RIM testing attenuation rate tomogram .............................................. 101

Figure 4.22 Comparison of the embedded geological anomalies and the reconstructed

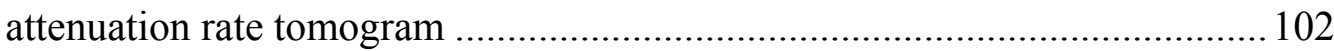

Figure 4.23 Reconstructed attenuation rate distribution in the physical model ............... 103

Figure 4.24 Relative error of the reconstructed attenuation rate in the physical model..... 104 


\section{CHAPTER 1 INTRODUCITON}

\subsection{Geological Anomalies in Longwall Mining}

The Longwall mining method has been widely used in coal mines. The production of longwall mines accounts for about $52 \%$ of underground coal production in US. The cutting speed of the shearing drum is up to $40 \mathrm{fpm}(12.2 \mathrm{~m} / \mathrm{min})$, and the daily production is up to 57,000 raw (U.S.) tons (Peng, 2006).

Of all of the engineering disciplines, mining engineers have the most challenging construction materials with which to deal. Mining engineers must deal with rock materials as they exist in their natural states and design mine structures without well-known and defined properties, as opposed to other engineering disciplines in which the construction materials are man-made and their properties are well-known in advance. Many roof control techniques developed by researchers are primarily applicable to the geological conditions that were assumed in the development of those techniques, and variation of rocks or rock properties may require modification or significant redesign of the support technology. In other words, a change in the geologic properties often results in significant variation of the rock behavior so that the original design criteria are no longer suitable. When this occurs, those techniques may not work, and in the worst case, failure of the support system may result (Peng, 2008). For example, sandstone channels that dip into the coal seam are normally very hard and reduce the minable seam height. The regular drum cutter bits are designed for coal and cannot cut hard sandstone effectively. If the sandstone intrusion penetrates too deep into the coal, the face equipment will not be able to advance, and has to be relocated. So, the production will slow down and even stop. 
Unexpected geological anomalies in the coal seam not only affect the productivity, but also affect the safety in the mines. Therefore, it is important to know as much as possible about the depositional environments that exist on the mine property or sections of the mine property.

In addition to sandstone channels, there are many other kinds of geological anomalies in front of the longwall panel, such as dikes, faults, and rock partings etc. (Figure 1.1), all the geological anomalies will affect the cutting speed, productivity, and safety of mining operations if they were not detected in advance.

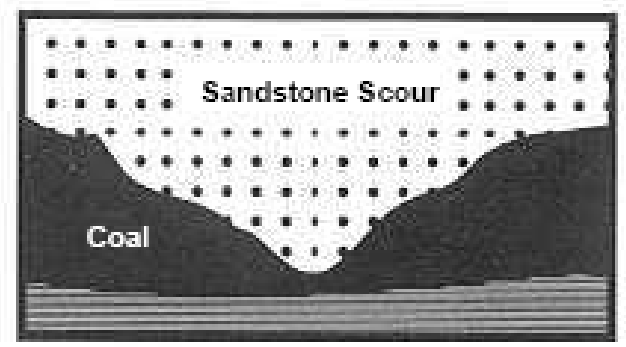

Washout

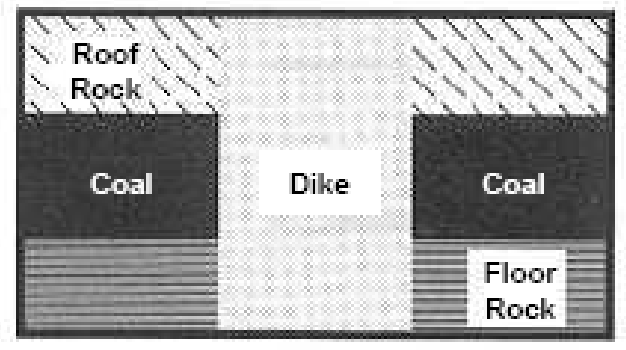

Dike

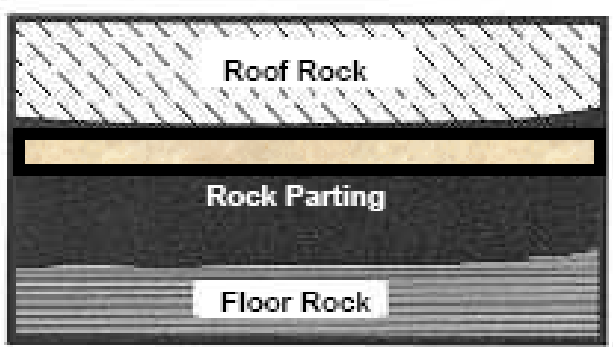

Interbedding

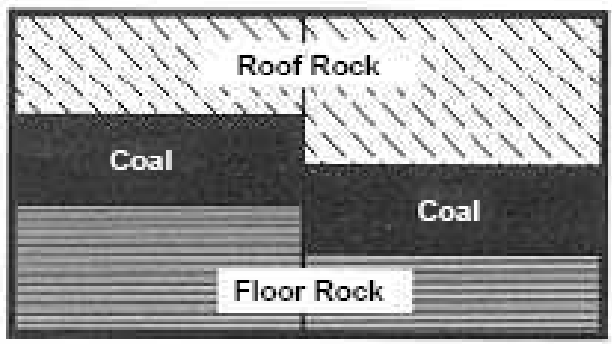

Fault

Figure 1.1 Geological anomalies in front of Longwall panel (Stolarczyk and Fry, 1990)

\subsection{Radio Imaging Method (RIM)}

In order to detect those geological anomalies in front of longwall face, the Radio Imaging Method (RIM) has been developed and considered to be the most promising geophysical method for detecting the geological anomalies in a longwall panel because of large 
penetration range and the ability to construct a 3-D model of the geological anomalies from the survey data (Stolarczyk and Fry, 1990).

RIM was developed in the early 1980s to detect/image hazards and obstructions in coal panels prior to longwall mining. Fry and Doe undertook the first RIM tomogram surveys in 1985 and 1986 (Stolarczyk, et al., 1985, 1986, 1990). Since then, RIM has been used extensively in the United States, Australia, New Zealand, South Africa, and the United Kingdom to evaluate coal seam geology (Stolarczyk and Peng, 2003).

The principle of RIM is the measurement of the attenuation of a medium-frequency $(30-1000 \mathrm{kHz})$ continuous electromagnetic (EM) wave radio signal that has been transmitted through a panel of rock or coal. A transmitting antenna (TX) which generates the EM signal is placed in the headgate, and a receiving antenna (RX) which receives the EM signal is put in the tailgate (Figure 1.2). In a homogeneous coal seam, the electromagnetic wave (radio signal) is attenuated by the materials with distance traveled at a fixed rate which is termed the attenuation rate. Since different rock types or geological structures have different EM properties (dielectric constant and electrical conductivity, etc.), the attenuation rates are different from the coal seam. If a geological anomaly exits along the EM propagation ray path, the receiving antenna will measure lower signal strength (increased attenuation rate). Changes in the attenuation rate from the background level indicate conductivity anomalies which can then be related to geology.

As an example: if a $100-\mathrm{kHz}$ radio wave travels $100 \mathrm{ft}$ in a coal seam with a normal attenuation rate of $18 \mathrm{~dB} / 100 \mathrm{ft}$, it decays by $18 \mathrm{~dB}$. If the radio wave must travel through a 20 - $\mathrm{ft}$-wide water-filled entry, it will decay at a rate of $45 \mathrm{~dB} / 100 \mathrm{ft}$ over those $20 \mathrm{ft}$ instead of the coal-based rate. The difference between the coal rate and the water rate is $27 \mathrm{~dB} / 100$ 
$\mathrm{ft}$ and its effect on the attenuation is an additional $5.4 \mathrm{~dB}$ (excess loss $=20 \mathrm{ft} \times(0.45-0.18)$ $\mathrm{dB} / \mathrm{ft}=5.4 \mathrm{~dB}$ ). Therefore, the total signal loss for the radio wave is $23.4 \mathrm{~dB}$ instead of 18 $\mathrm{dB}$ due to the presence of the water-filled entry (a 30\% increase for a $100 \mathrm{ft}$ signal path) (Stolarczyk and Peng, 2003).

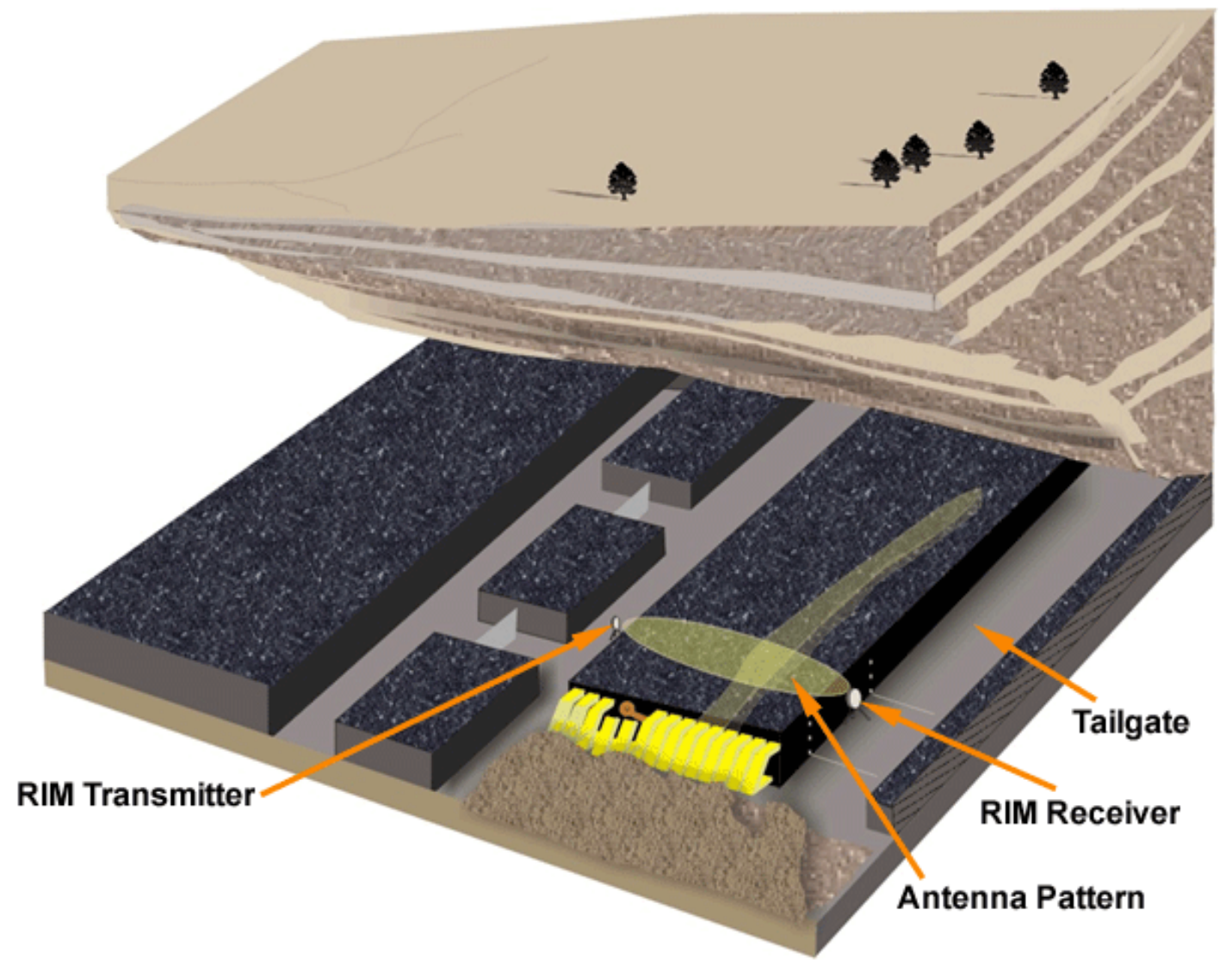

Figure 1.2 Layout of RIM working condition and instrumentation (Stolar Horizon, Inc., 2004)

For safety reason, in the RIM testing procedure, the transmitter will be fixed in the headgate, and the receiver will move along the tailgate and the signals are monitored at different locations. Equal numbers of equally-spaced TX and RX survey points are laid across the survey area. After that, the transmitter will be moved to the adjacent point and conduct the same testing procedure till all the testing points are done (Figure 1.3). The RX unit measures each TX broadcast, creating ray paths of radio-wave signal through the 
longwall block. Each ray path provided a measurement of receives signal strength unique to the origin and termination of the ray path and the condition of the coal seam through which the signal propagated. After the RIM test is finished, the longwall panel geological tomogram can be reconstructed employing some popular reconstruction algorithms methods including: the algebraic reconstruction technique (ART) (Gordon, 1974), the simultaneous iterative reconstruction technique (SIRT) (Gilbert, 1972), and the back projection technique (BPT) (Kuhl, 1963). Figure 1.4 shows the survey ray path density and the attenuation rates tomographic image (tomogram) using the SIRT algorithm for a longwall panel at the 290-kHz RIM test (Stolarczyk et al., 2006). The coolest color (blue) represents the lowest attenuation rate sampled while the hottest color (red) represents the highest attenuation rate.

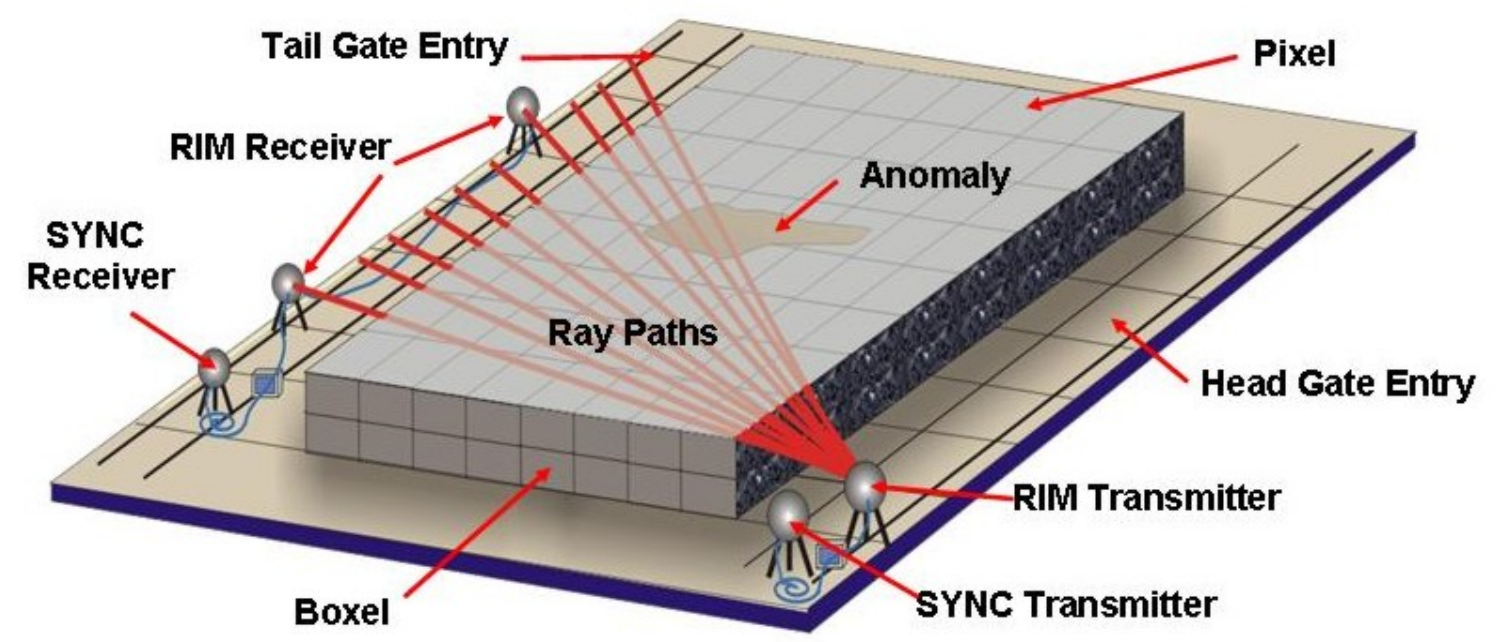

Figure 1.3 RIM testing procedure (Stolarczyk et al., 2006)

Stolarczyk $(1984,1986)$ developed instrumentation and survey methods to determine seam continuity and image anomalous geologic structures ahead of the working face. The first survey was carried out in a Utah Power and Light Company mine in central Utah 
$(1985,1986)$. In the last couple decades, a lot of RIM surveys have been done, and reports and papers have been published (Stolarczyk et al., 1999, 2003, 2004, 2006; Stolar Horizon, Inc., 2004; Thomson, 1993; Shope, 1987; Wu, 1988 and Zhao, 2005).

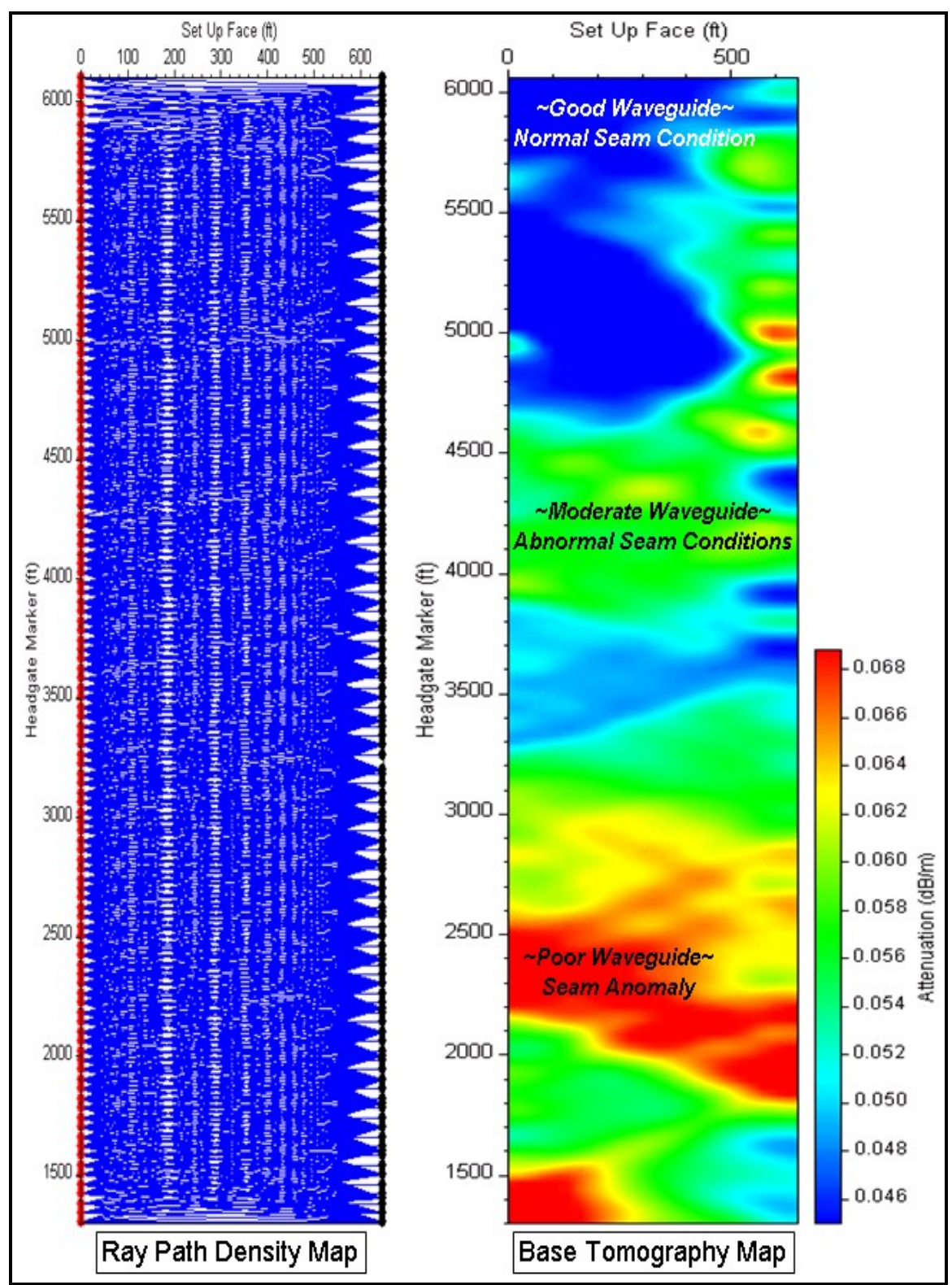

Figure 1.4 Ray path plot and tomogram at a longwall panel with $290-\mathrm{kHz}$ RIM test (Stolarczyk et al., 2006)

The latest technology is RIM-IV which was developed by Stolar Research Corporation. The RIM-IV instrumentation consists of a transmitter (TX) unit and a 
companion receiver $(\mathrm{RX})$ unit as shown in Figure 1.5. The in-mine instrumentation is small, light-weight, and portable. The National Mining Association (NMA) and the U.S. Department of Energy (DOE's) Industry of the Future Mining program provided research and development funds to extend the RIM instrumentation operating range to greater than 3,000 feet (Stolarczyk et al., 2006).

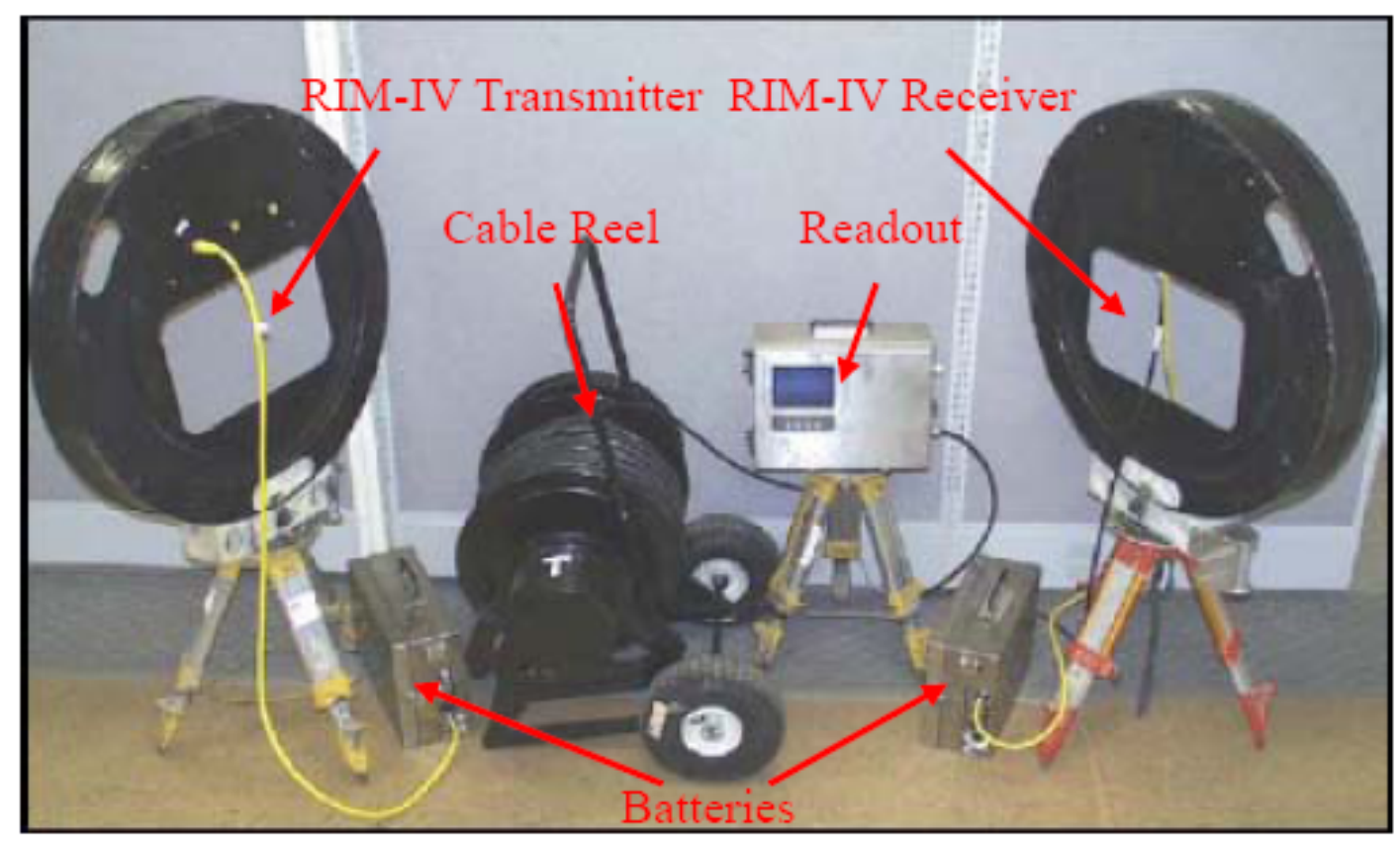

Figure 1.5 RIM-IV Hardware (Stolarczyk and Peng, 2003)

In general, the process for surveying a longwall panel includes five steps:

(1) Plan the survey;

(2) Collect the field data (which includes equipment calibration to the specific seam, a reconnaissance scan, and tomographic scan as needed);

(3) Analysis of the data, which includes construction of 2-D or 3-D images;

(4) Confirmation as required (in some cases drilling into anomalies to confirm targets); and

(5) Integration of geologic intelligence into mine planning. 


\subsection{Problem Statement}

By using low frequency EM signals, the RIM technology is the most promising geophysical tool for exploring the geological anomalies ahead of the modern longwall faces which are normally wider than 1,000 ft. However, when a number of anomalies co-exist in an area (very common), it becomes difficult for the RIM technology to differentiate the contributions of each individual anomalous factor to the total attenuation. This will affect the interpretation accuracy of the RIM technology.

In order to increase the accuracy of RIM technology, physical modeling provides a useful method to investigate the capabilities and limitations of RIM technology. However, there is no past research regarding how to employing the laboratory method to study RIM. In order to develop a laboratory method to study RIM, some important questions must be answered:

1. What are the scale factors to be used in the scale models?

2. What are the materials to be used to construct the scale model, and what are their electromagnetic properties (dielectric constant, electrical conductivity, permeability, etc.)?

3. What is the equipment to be used to test the scale model in the laboratory, and how to test it?

4. What is the software to be used to process the testing data and generate the tomogram?

5. Will the reconstructed tomogram match the characteristics (attenuation rate, location, and shape etc.) of the embedded geological anomalies? 


\subsection{Objectives}

The main objective of this study is to develop a useful laboratory physical modeling method to study the resolution limits of RIM technology. The detail of the objective includes:

(1) Scale factors analysis and scale model design. Based on the physical modeling simulation theory, the scale factors, including the dimension scale factor, dielectric constant scale factor, and electrical constant scale factor, etc., will be analyzed. Based on the results of the analysis, the dimension of the scale model will be designed too.

(2) EM properties (dielectric constant and electrical conductivity) testing. In the scale models, different mixture ratios of sand, gypsum, cement, and salt will be used to build the floor and roof strata, and a mixture of coal powder and gypsum will be used to simulate the coal seam. The EM properties will be tested at different mixture ratios and water content. The proper mixture materials will be selected to build the scale model.

(3) Testing equipment and testing procedure design. High frequency ground penetrating radar which contains separated transmitter and receiver will be used in the laboratory test. Calibration test, reconnaissance test, and RIM testing procedures will be properly designed.

(4) RIM tomogram software development. There is no RIM tomogram software available for this study. Based on the EM wave propagation theory in the coal seam, RIM tomogram software will be developed in Matlab to reconstruct the attenuation rate tomogram. The basic algorithms employed to develop the software are Algebraic Reconstruction Technique (ART) and Simultaneous Iterative Reconstruction Technique (SIRT). 
(5) RIM surveys on the scale model and tomogram reconstruction. After the calibration test, reconnaissance test, and RIM testing have been performed, the testing data will be used to generate the tomograms which in turn will be compared with the embedded geological anomalies.

The detailed flow chart of the laboratory physical model RIM study methodology is shown in Figure 1.6.

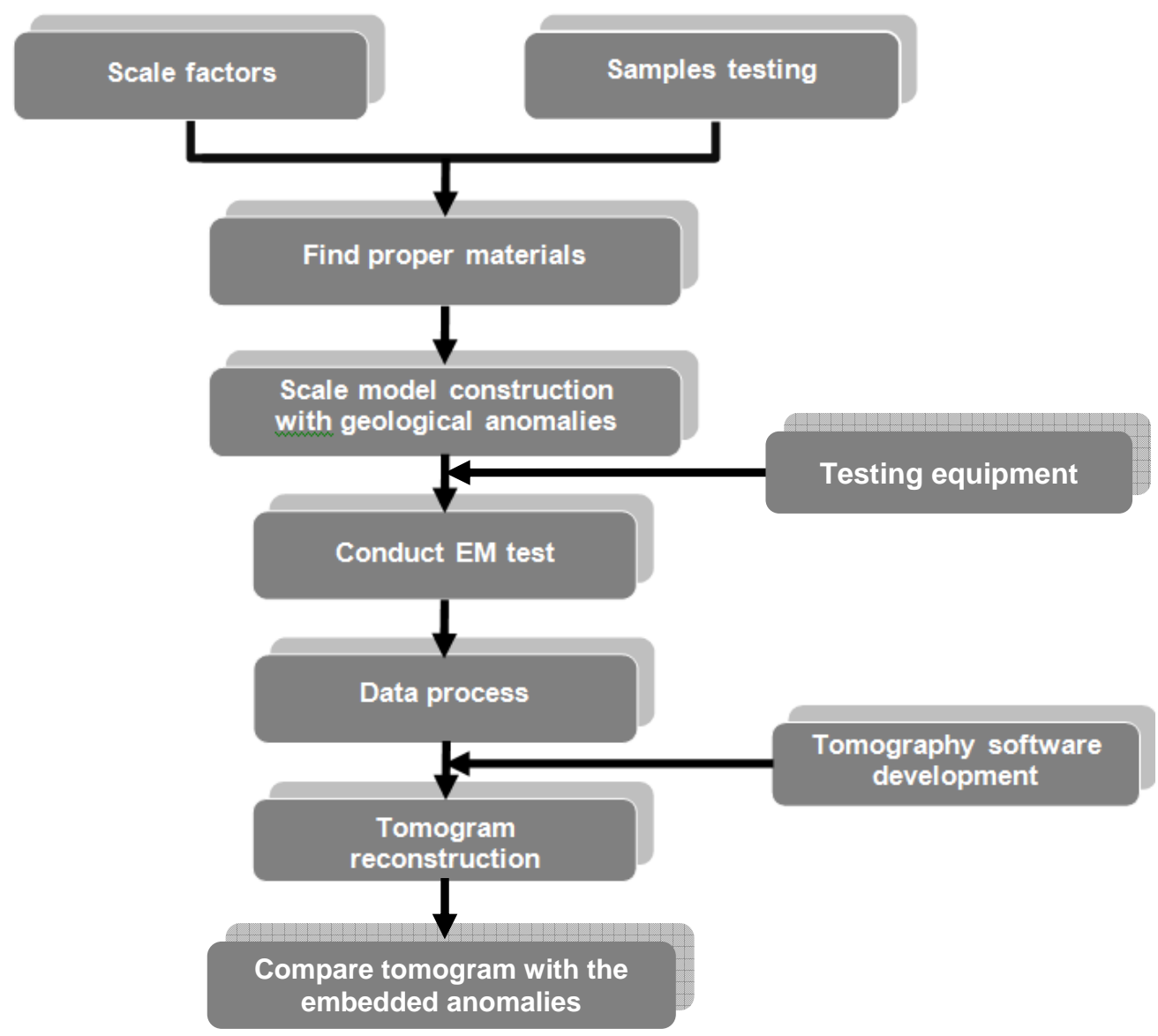

Figure 1.6 Laboratory RIM study methodology

\subsection{Scope}

This dissertation is divided into six chapters. Chapter 1 is an introduction. Chapter 2 is a literature review including the electrical properties (dielectric constant and electrical 
conductivity) of rock and coal, RIM and Electromagnetic (EM) Seam Wave, RIM tomography method, and EM scale model theory. Chapter 3 presents EM properties testing results with different mixture samples. The testing method used in the laboratory and data process is introduced. MiniRIM attenuation rate tomogram software development and validation is presented too. Chapter 4 presents the results of two physical models as well as data analysis. Conclusions and recommendation are presented in chapter 5 and 6 , respectively. 


\section{CHAPTER 2 LITERATURE REVIEW}

\subsection{Electrical Properties of Rock/Coal}

RIM technology uses changes of attenuation rate of electromagnetic (EM) wave to detect geological anomalies in front of the longwall face. The attenuation rate of propagating EM wave through different geological material is not only frequency dependent, but also depends on the electrical properties (dielectric constant, electrical conductivity, magnetic permeability, and attenuation rate) of the rocks/coal under investigation. For this study, it is very important to understand the electrical properties of rock and coal.

\subsubsection{Dielectric Constant (DC) /Permittivity}

A material is classified as "dielectric" if it has the ability to store energy when an external electric field is applied. The dielectric constant of a material is equivalent to relative permittivity $\left(\varepsilon_{r}\right)$ or the absolute permittivity $\left(\varepsilon^{*}\right)$ relative to the permittivity of free space $\left(\varepsilon_{o}\right)$ (Eq. 2-1) (Agilent).

$$
\varepsilon=\varepsilon_{r}=\frac{\varepsilon^{*}}{\varepsilon_{o}}=\varepsilon_{r}^{\prime}-j \varepsilon_{r}^{\prime \prime}
$$

where $\varepsilon_{r}^{\prime}$ is real permittivity $(F / m), \varepsilon_{r}^{\prime \prime}$ is imaginary permittivity $(F / m), \varepsilon_{r}$ is complex relative permittivity $(F / m), \varepsilon_{o}=10^{-9} / 36 \pi(F / m)$, and $j=\sqrt{-1}$.

The real part of permittivity $\left(\varepsilon_{r}^{\prime}\right)$ is a measure of how much energy from an external electric field is stored in a material. The imaginary part of permittivity $\left(\varepsilon_{r}^{\prime \prime}\right)$ is called the loss factor and is a measure of how dissipative or lossy a material is to an external electric field. The imaginary part of permittivity $\left(\varepsilon_{r}^{\prime \prime}\right)$ is always greater than zero and is usually 
much smaller than $\left(\varepsilon_{r}^{\prime}\right)$. The loss factor includes the effects of both dielectric loss and conductivity.

The dielectric constant is a critical EM wave parameter because it controls the propagation velocity of electromagnetic waves through a material and the reflection coefficients at interfaces, as well as affecting the vertical and horizontal imaging resolution. Therefore, knowing the dielectric constant values of materials helps in planning EM surveys and in better understanding and interpreting EM images.

There are different methods to measure dielectric constant: the coaxial probe method, the transmission line method, and the parallel plate method, etc. For solid/powder dielectric materials, the coaxial probe method is most popular.

A typical dielectric constant measurement system using a coaxial probe method consists of a network or impedance analyzer, a coaxial probe and software. Both the software and the probe are included in the $85070 \mathrm{E}$ dielectric probe kit. An external computer is needed in many cases to control the network analyzer through the GPIB. The 82357A USB to GPIB interface provides a convenient and flexible way to realize this connection. The combination of a network analyzer and the probe kit is shown in Figure 2.1.

The analyzer measures the reflection coefficient between the probe kit and the material under test by transmitting the electromagnetic energy through the electrode of the probe kit. Then the algorithm software calculates the dielectric constant of the test material and allows direct readings for the real part $\left(\varepsilon_{r}^{\prime}\right)$ and the imaginary part of $\left(\varepsilon_{r}^{\prime \prime}\right)$ the constant. The typical accuracy of the measured dielectric constant according to the manufacturer is $\pm 5 \%$ for the permittivity as well as for the loss factor (Agilent, Technical Overview). In addition, the software calculates the loss tangent (The loss tangent or $\tan \delta$ is defined as the 
ratio of the imaginary part of the dielectric constant to the real part, $\tan \delta=\varepsilon_{r}^{\prime \prime} / \varepsilon_{r}^{\prime}$ ) for the test material. Calibration of the instrument before the beginning of the measurements is required. Air, short, and water compensations are recommended before the measurement. Air compensation is performed by leaving the dielectric probe opens to air. A short compensation is performed by connecting a shorting block to the probe, and finally water compensation is done by inserting the electrode in water. It should be noted that the water for the water compensation should be deionized and at a temperature of $25^{\circ} \mathrm{C}$ (about $77^{\circ} \mathrm{F}$ ). After the calibration has been performed, the measurement can start. For this purpose, the frequency range that is of particular interest should be pre-set in the software as mentioned previously. The number of frequency points that will be tested should be estimated so they could also be pre-set in the tested frequency range.

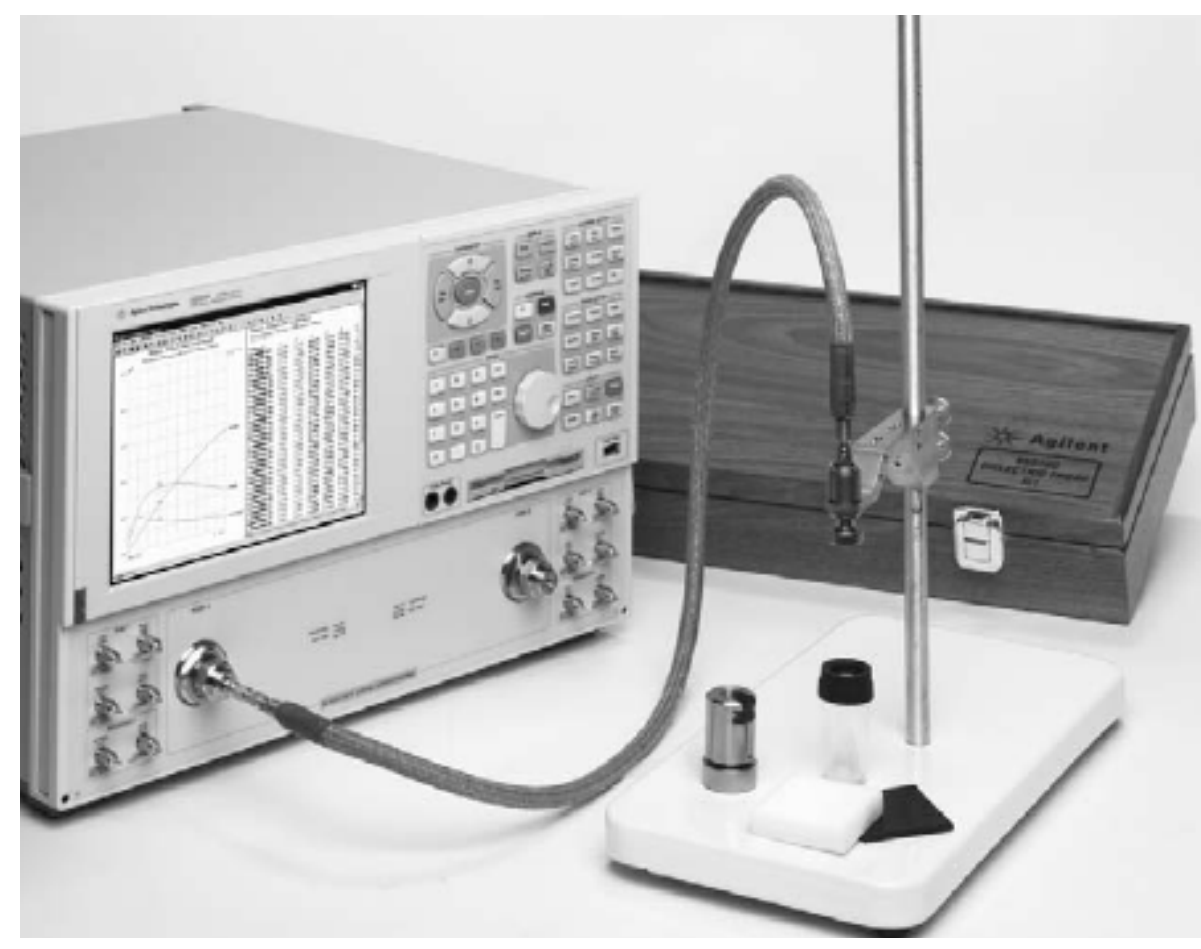

Figure 2.1 Dielectric constant measurement equipment (network analyzer and probe kit) (Agilent) 
Measured dielectric-constant values for various rocks and minerals may be found in the literatures (e.g., Parkhomenko, 1967, Church, et al., 1985, Marland and Rowson, 2001, Marquina and Martinez, 2003, Davis and Annan, 1989; Daniels, 1996; Olhoeft, 1989; Schon, 1996; Ulaby et al., 1990). Some reported bulk dielectric constant values of common earth materials are presented in Table 2.1. Actually, bulk dielectric constants of rocks and sediments reflect complex mixtures of materials and architectures that vary from one rock lithology to the next. In rocks and sediments, dielectric properties are primarily a function of mineralogy, porosity, water saturation, frequency, component geometries, and electrochemical interactions (Knight and Endres, 1990; Knoll, 1996).

Table 2.1 Bulk dielectric constants (measured at $100 \mathrm{MHz}$ ) of common earth materials

\begin{tabular}{|c|c|c|}
\hline Material & From Davis and Annan, & From Daniels, 1996 \\
\hline Air & $\underline{\mathbf{1 9 8 9}}$ & 1 \\
\hline Fresh water & 80 & 81 \\
\hline Sandstone, dry & & $2-3$ \\
\hline Sandstone, wet & & $5-10$ \\
\hline Limestone & $4-8$ & 7 \\
\hline Limestone, dry & & 8 \\
\hline Limestone wet & & $6-9$ \\
\hline Shales & $5-15$ & \\
\hline Shale, wet & & $2-6$ \\
\hline Silts & $5-30$ & $15-40$ \\
\hline Clays & $5-40$ & 3.5 \\
\hline Clay, dry & & 8 \\
\hline Clay, wet & & $4-6$ \\
\hline Coal, dry & & $15-30$ \\
\hline Coal, wet & & $4-6$ \\
\hline Soil, sandy dry & & $10-15$ \\
\hline Soil, sandy wet & & 5 \\
\hline Soil, clayey dry & & 7 \\
\hline Soil, clayey wet & & $4-7$ \\
\hline Granite & & \\
\hline Granite, dry & & \\
\hline Granite, wet & & \\
\hline Salt, dry & & \\
\hline
\end{tabular}


A Time-Propagation (TP) dielectric mixing model can be used to describe how dielectric constant is affected by different minerals, porosity, and water saturation (Martinez and Byrnes, 2001). A four-dimensional representation of the TP-model at a given frequency is shown in Figure 2.2. The matrix mineralogy axis $(\mathrm{Xm})$ is the dielectricconstant value of the matrix components at zero porosity and water saturation. The locations of quartz, mica, and calcite are indicated on the Xm axis. For most earth materials, porosity ranges from $0 \%$ to $50 \%$, and water saturation ranges from $0 \%$ to $100 \%$. Calculated bulk dielectric-constant values range from greater than 4 to less than 28 .

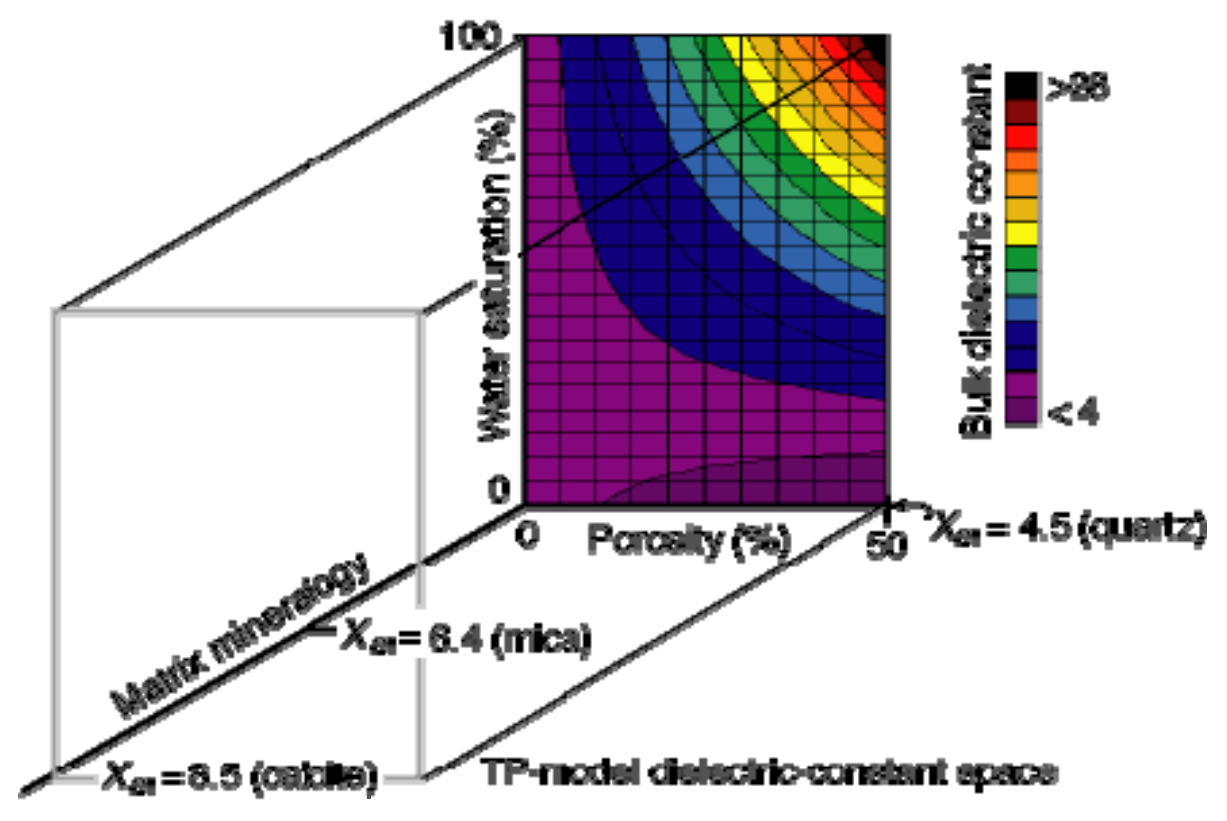

Figure 2.2 Four-dimensional representation of the TP-model input parameters (matrix mineralogy, porosity, and water saturation) and resulting bulk dielectric constant values (Martinez and Byrnes, 2001)

Figure 2.3 shows the dielectric constant testing results at different moisture content and frequency for the coal samples of the Sewickley Seam (Boykov, 2006). The testing results show that the dielectric constant decreases with increasing frequency (from $10 \mathrm{KHz}$ to $10 \mathrm{MHz}$ ), and increases with increasing moisture content. 


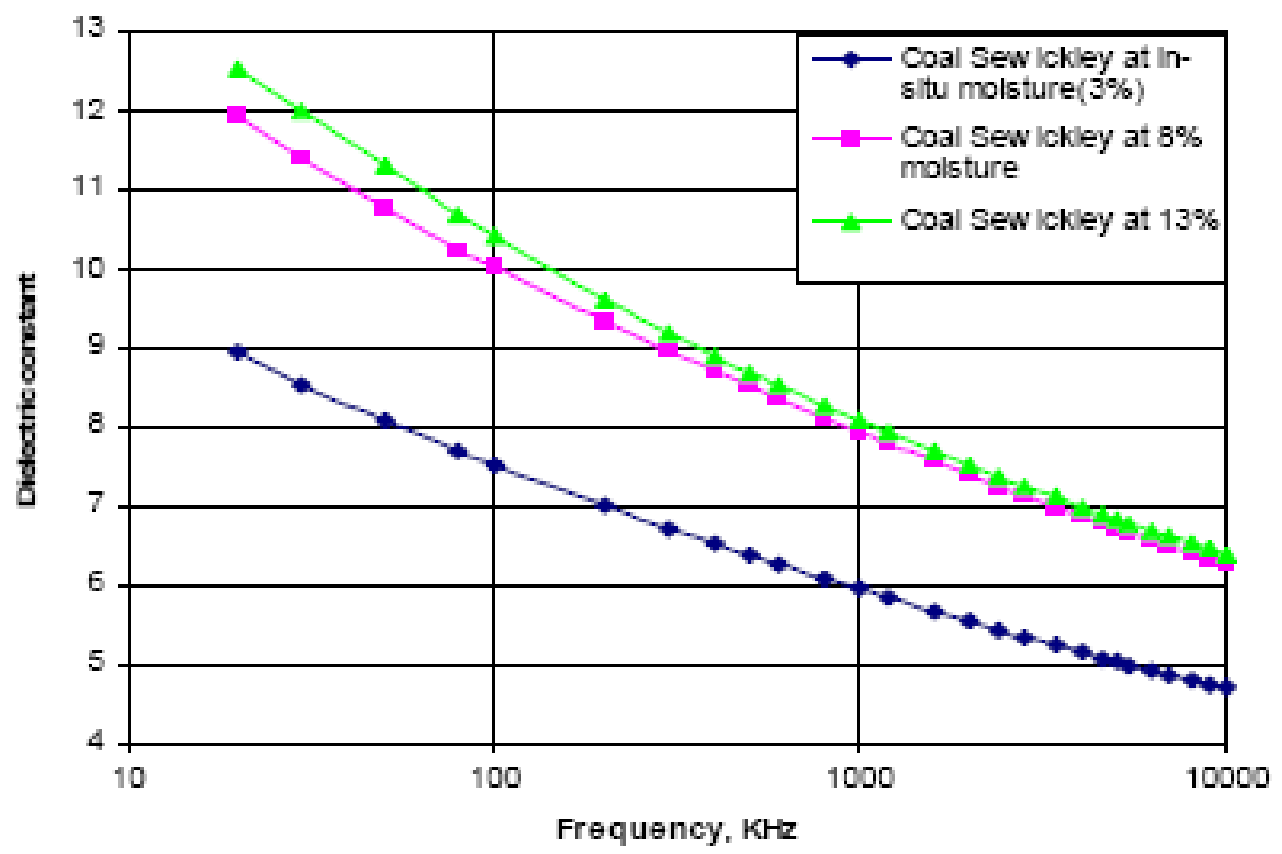

Figure 2.3 Comparisons of the dielectric constants at different moisture levels for a coal sample from the Sewickley Seam (Boykov, 2006)

\subsubsection{Electrical Conductivity (EC)/Electrical Resistivity (ER)}

Electrical conductivity is a measure of a material's ability to conduct an electric current. Electrical conductivity is the reciprocal (inverse) of electrical resistivity, and has the units of Siemens per meter $(\mathrm{S} / \mathrm{m})$. It is commonly represented by the Greek letter $\sigma$. The resistivity for some typical earth materials is shown in Table 2.2.

In general, electrical conductivity is not directly measured. It can be calculated by inverse of electrical resistivity. The electrical resistivity is calculated by (Boykov, 2006):

$$
R=\frac{1}{\omega \times \varepsilon_{r}^{\prime} \times \tan \delta \times 8.854 \times 10^{-12}}, \quad \text { ohm }-m
$$

where: $\omega=2 \pi f$ is the angular frequency of the EM wave, in radian per second, and $f=$ frequency in Hertz, $\varepsilon_{r}^{\prime}=$ the real part of the permittivity, $F / m$, and 
$\tan \delta=\varepsilon_{r}^{\prime \prime} / \varepsilon_{r}^{\prime}$ is the loss tangent.

So, the electrical conductivity,

$$
\sigma=\frac{1}{R}, \quad S / m
$$

The EC/ER of a dielectric material is largely affected by the composition of the materials, the water content, the frequency, and the temperature etc. (Anggoro et al., 2006). An example showing the effect of water content, salt, and carbon on resistivity at the frequency of $50 \mathrm{~Hz}$ is shown in Figure 2.4. The testing results show that the resistivity of soil decreases with increasing percentages of both of water and salt, and that changing the salt content has more effect on soil's resistivity.

Table 2.2 The electrical resistivity for some typical earth materials (Johnson, 2003)

\begin{tabular}{|c|c|c|c|c|c|c|c|c|c|c|c|c|c|}
\hline & $1 \Omega-m$ & & & $\begin{array}{c}10 \Omega- \\
\mathrm{m}\end{array}$ & & & $\begin{array}{c}100 \\
\Omega-\mathrm{m}\end{array}$ & & & $\begin{array}{l}1000 \\
\Omega-\mathrm{m}\end{array}$ & & & $\begin{array}{c}10000 \\
\Omega-\mathrm{m}\end{array}$ \\
\hline Clay and marl & $x$ & $x$ & $x$ & $x$ & $x$ & $x$ & & & & & & & \\
\hline & & & & & & & & & & & & & \\
\hline Loam & & & $x$ & $x$ & $x$ & & & & & & & & \\
\hline & & & & & & & & & & & & & \\
\hline Top soil & & & & & & $x$ & $x$ & & & & & & \\
\hline & & & & & & & & & & & & & \\
\hline Clayey soils & & & & & & & $x$ & $x$ & & & & & \\
\hline & & & & & & & & & & & & & \\
\hline Sandy soils & & & & & & & & & $x$ & $x$ & $x$ & & \\
\hline & & & & & & & & & & & & & \\
\hline Typical mine water & $x$ & $x$ & $x$ & $x$ & & & & & & & & & \\
\hline & & & & & & & & & & & & & \\
\hline Typical surface water & & & $x$ & $x$ & $x$ & & & & & & & & \\
\hline & & & & & & & & & & & & & \\
\hline Shale & & & & $x$ & $x$ & $x$ & & & & & & & \\
\hline & & & & & & & & & & & & & \\
\hline Limestones & & & & & & $x$ & $x$ & $x$ & $x$ & $x$ & & & \\
\hline & & & & & & & & & & & & & \\
\hline Sandstones & & & & & $x$ & $x$ & $x$ & $x$ & $x$ & $x$ & $x$ & $x$ & \\
\hline & & & & & & & & & & & & & \\
\hline Coal & & & & & & & & $x$ & $x$ & $x$ & $x$ & & \\
\hline & & & & & & & & & & & & & \\
\hline
\end{tabular}




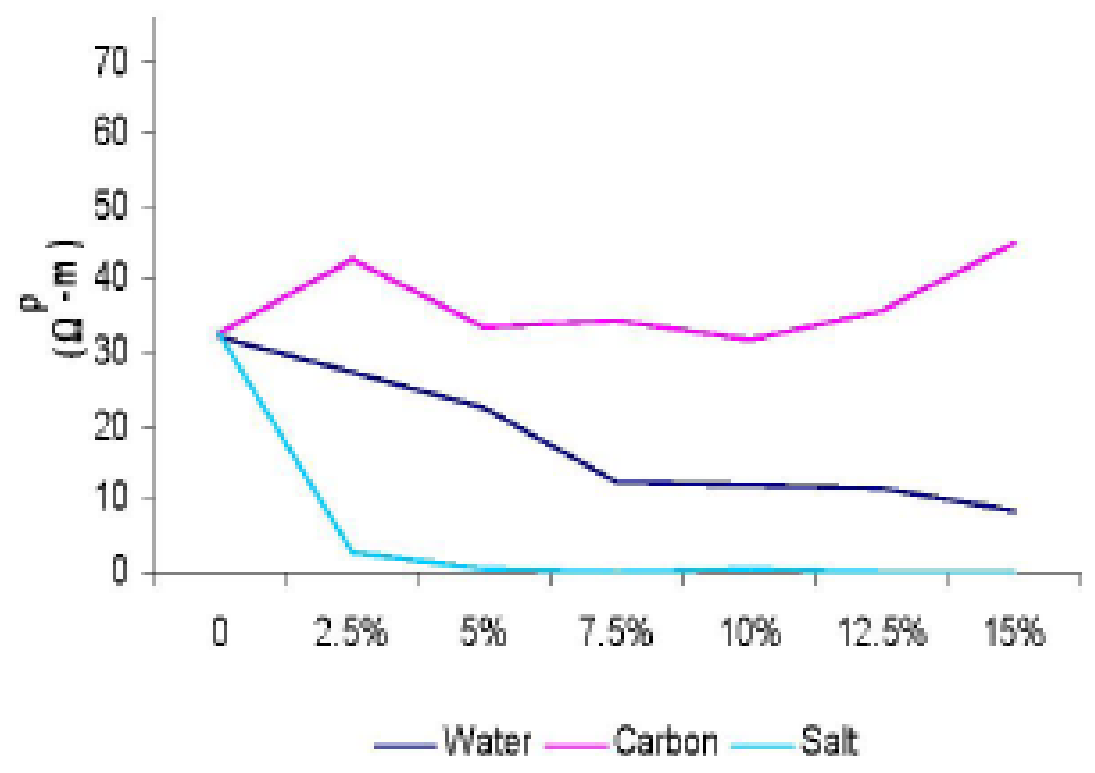

Figure 2.4 The effect of water, carbon and salt in soil on resistivity (Anggoro et al., 2006)

Boykov (2006) also tested the EM properties of rock and coal at different frequencies and moisture contents. Figure 2.5 shows the testing results of the resistivity of coal samples from the Sewickley Seam at different frequencies which shows that the resistivity decreases with the increasing frequency.

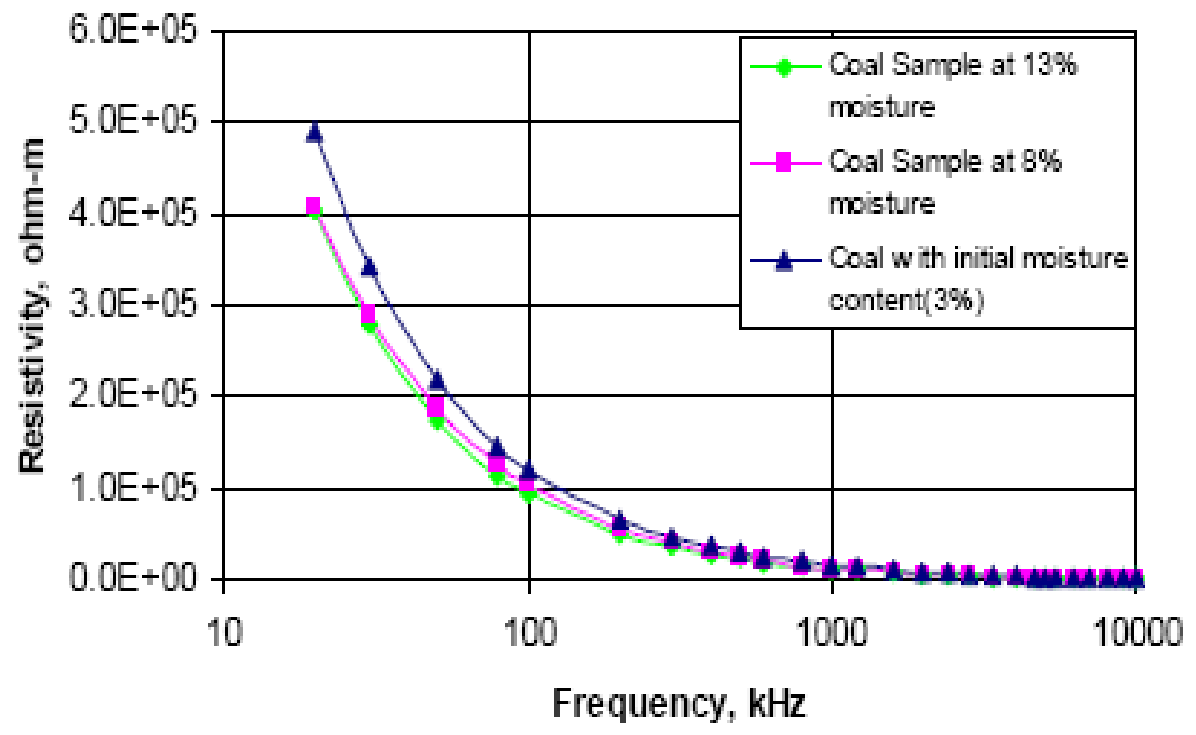

Figure 2.5 Comparisons of the electrical resistivity observed at different moisture levels for a coal sample from the Sewickley Seam (Boykov, 2006) 
In general, the electrical conductivity in rock is greater than the electrical conductivity in the coal seam. EM wave energy propagation occurs in the seam because the higher conductivity sedimentary rock surrounding the seam forces the radio wave energy to flow in the coal seam between the sedimentary rocks (Stolarczyk and Fry, 1990). The electrical conductivity effect on the attenuation rate will be discussed in the following section.

\subsubsection{Magnetic Permeability}

Magnetic permeability $(\mu)$ describes the interaction of a material with a magnetic field. For free space, the magnetic permeability is denoted by $\mu_{o}$ and is equal to $4 \pi \times 10^{-7} \mathrm{H} / \mathrm{m}$. The relative permeability of any material is the ratio of the permeability of the material to that of free space. It is, therefore, a dimensionless quantity and is denoted by $\mu_{r}$. Some materials such as iron (ferrites), cobalt, nickel, and their alloys have appreciable magnetic properties; however, many materials are nonmagnetic, making the permeability very close to the permeability of free space $\left(\mu_{r}=1\right)$.

\subsubsection{Attenuation Rate and Phase Shift}

Attenuation rate $(\alpha)$ is the rate of diminution of average power with respect to distance along a transmission path. The effect of attenuation in the coal seam waveguide is to reduce the magnitude of the EM wave along the ray path. Heaviside (Nahin, 1988) gave a formula for determining the attenuation rate as functions of frequency and the electrical parameters of the natural media:

$$
\alpha=\omega\left[\frac{\mu \varepsilon}{2}\left(\sqrt{1+\frac{\sigma^{2}}{\varepsilon^{2} \omega^{2}}}-1\right)\right]^{0.5} \quad \text { Nepers/meter }
$$


where: $\omega=2 \pi f$ is the angular frequency of the EM wave, in radian per second, and $f=$ frequency in Hertz, $\sigma=$ the electrical conductivity in Simens/meter $(\mathrm{S} / \mathrm{m})$, $\varepsilon=\varepsilon_{r} \varepsilon_{o}$ is the permittivity. The permittivity of free space $\varepsilon_{o}=\frac{1}{36 \pi} \times 10^{-9}$, Farads/meter, and $\varepsilon_{r}$ is the relative dielectric constant, and $\mu=\mu_{r} \mu_{o}$ is the magnetic permeability. The permeability of free space $\mu_{o}=4 \pi \times 10^{-7}$, and $\mu_{r}$ is the relative permeability.

From Eq. (2-3), the angular frequency, magnetic permeability, dielectric constant, and electrical conductivity have an effect on the attenuation rate.

Figure 2.6 illustrates the attenuation rate as a function of frequency of a non-magnetic medium with a relative dielectric constant of 6 . From this figure, the attenuation rate will apparently increase with a increase in frequency. Since shale or clay has a higher electrical conductivity than coal, the attenuation rate is also higher. In the higher frequency range $(10-1000 \mathrm{MHz})$, the difference of attenuation rate is even larger than at the low frequency range (0.1-10 MHz). So, a higher electrical conductivity has a higher attenuation rate. That is why the geological anomalies (sandstone intrusions, rock partings, faults, etc.) will apparently affect the attenuation rate in the coal seam and that is also the basis of the RIM technology.

The effect of the sedimentary rock at the boundary of the coal seam on attenuation rate is shown in Figure 2.7 (Stolarczyk and Peng, 2003). Under sandstone sedimentary rock or fractured shale, the attenuation rate increases because more of the RIM signal travels vertically into the boundary rock, i.e., leaks from the waveguide. If water is injected into the coal from an overlying paleochannel, then clay in the coal causes the electrical 
conductivity and attenuation rate/phase shift to increase. If the electrical conductivity is very large (shale), the larger electrical conductivity difference between the coal seam and the boundary rock make less energy leak into the boundary rock and therefore less attenuation in the coal seam.

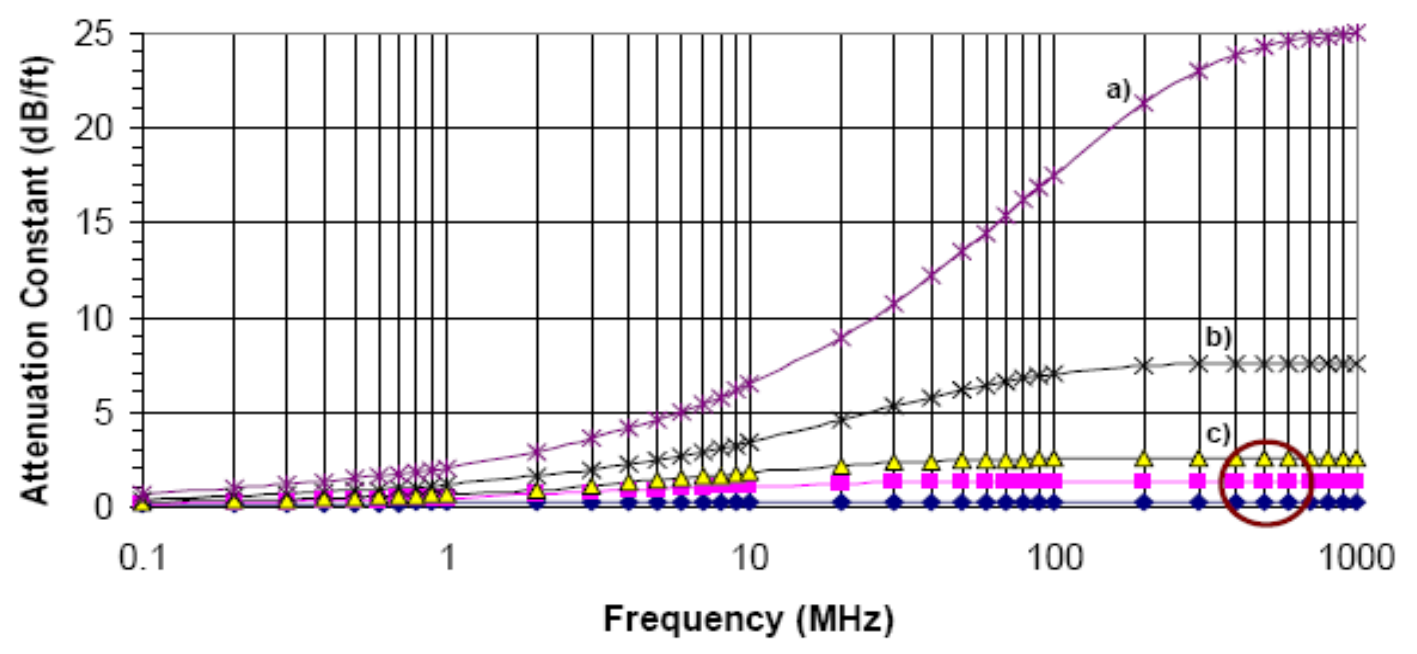

Figure 2.6 Attenuation rate (dB/ft) versus frequency exhibited by: a) shale/clay, b) coal with high moisture, c) range for US coals (Stolarczyk and Peng, 2003)

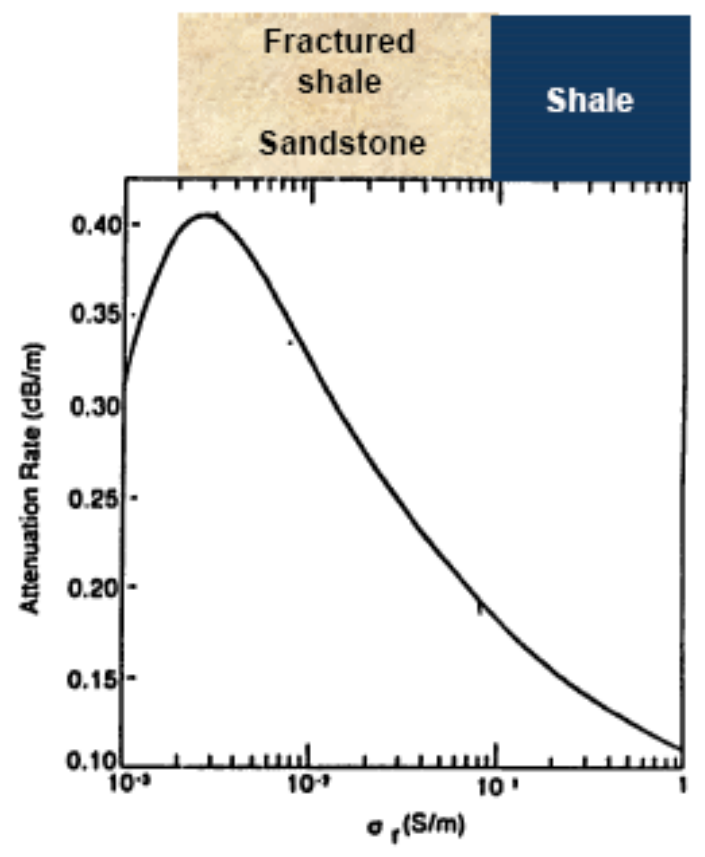

Figure 2.7 Coal seam EM wave attenuation rate versus boundary rock conductivity (Stolarczyk and Peng, 2003) 
Phase shift is a change in phase of a wave form between two points, expressed as degrees of lead or lag. For example, if a receiving antenna is moved a distance (d) from its original location to a new location, the reproduced waveform would be mathematically represented by (Stolarczyk and Peng, 2003):

where

$$
e m f=M \cos [\omega t+\theta]
$$

$e m f=$ electromotive force voltage waveform,

$M=$ the magnitude of the sine wave signal,

$t=$ the continuing time, and

$\theta=d\left(\frac{2 \pi}{\lambda}\right)$ is the phase shift (or rotation angle) in radians and one radian is

57 electrical degrees.

The attenuation rate/phase shift rapidly increases with decreasing seam height (Figure 2.8). These curves suggest that the propagation constants are inversely related to seam height. The thinning of coal seam can be easily detected with RIM. A higher attenuation rate zone suggests that the coal seam boundary rock is changing; the seam is rapidly thinning, and/or water has been injected into the coal seam. The increase in attenuation rate also can be explained from an EM wave energy flow point of view (Figure 2.9). When the seam height decreases, the energy density in the coal increases. Heating increases the dissipation of the radio wave per unit of travel distance (Stolarczyk and Peng, 2003).

The attenuation rate and phase shift increase with electrical conductivity of the coal seam as illustrated in Figure 2.10. The attenuation rate and phase shift are highly dependent on coal seam electrical conductivity. As discussed before, the electrical conductivity is affected by moisture content and composition of the materials etc. On the other hand, seam anomalies cause local regions of the coal seam conductivity to increase and become highly 
responsive targets. For example, faults and shear zones allow water to be injected into the coal seam. Rock partings can form a moisture barrier in the coal seam (Stolarczyk and Fry, 1990).

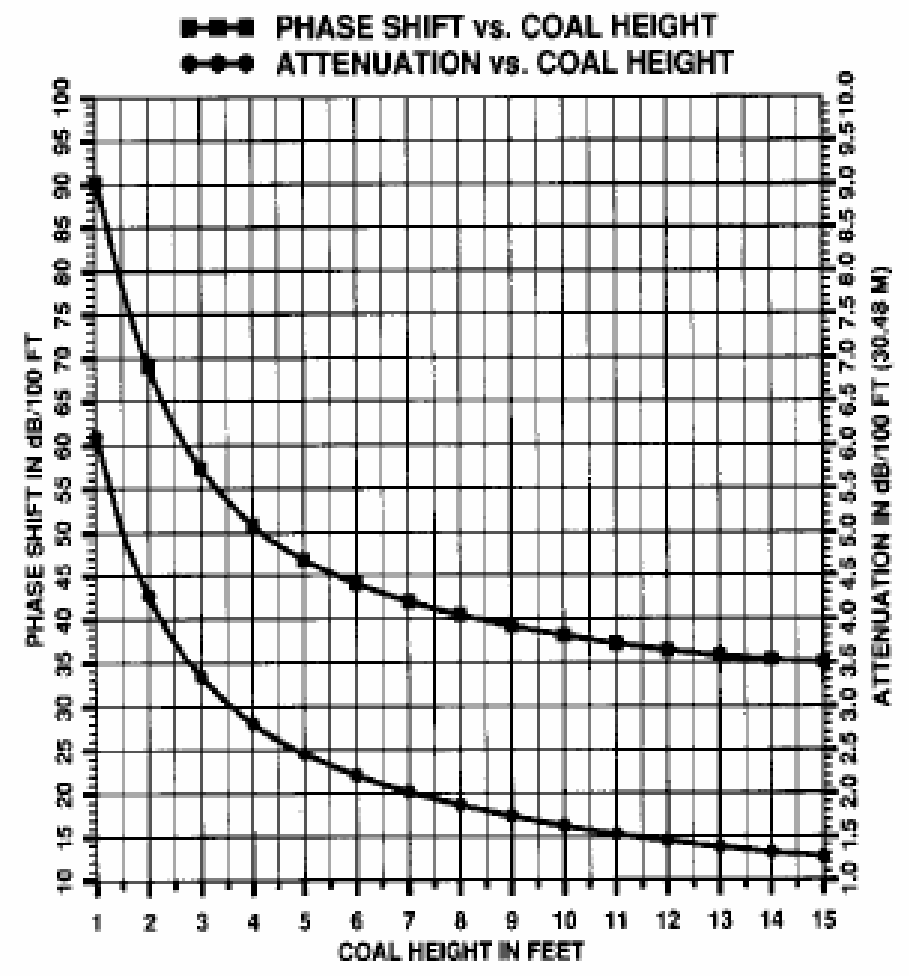

Figure 2.8 Sensitivity of radio waves to changes in coal layer thickness (Stolarczyk and Fry, 1990)

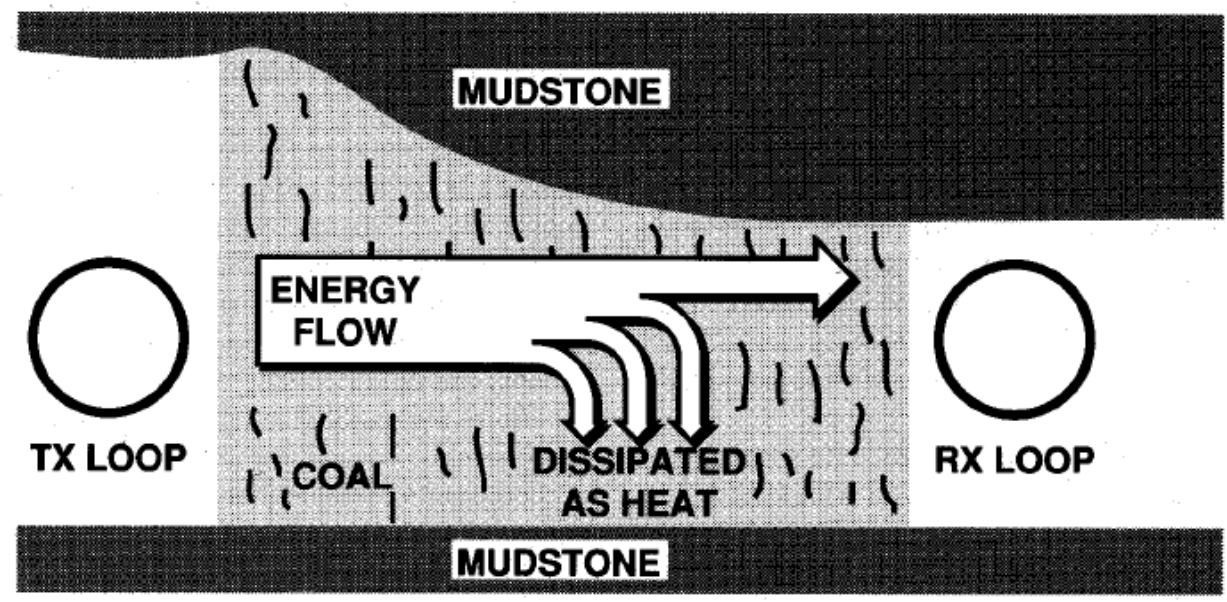

Figure 2.9 Energy flow along the ray path from the ransmitting (TX) to Receiving (RX) antenna (Stolarczyk and Fry, 1990) 


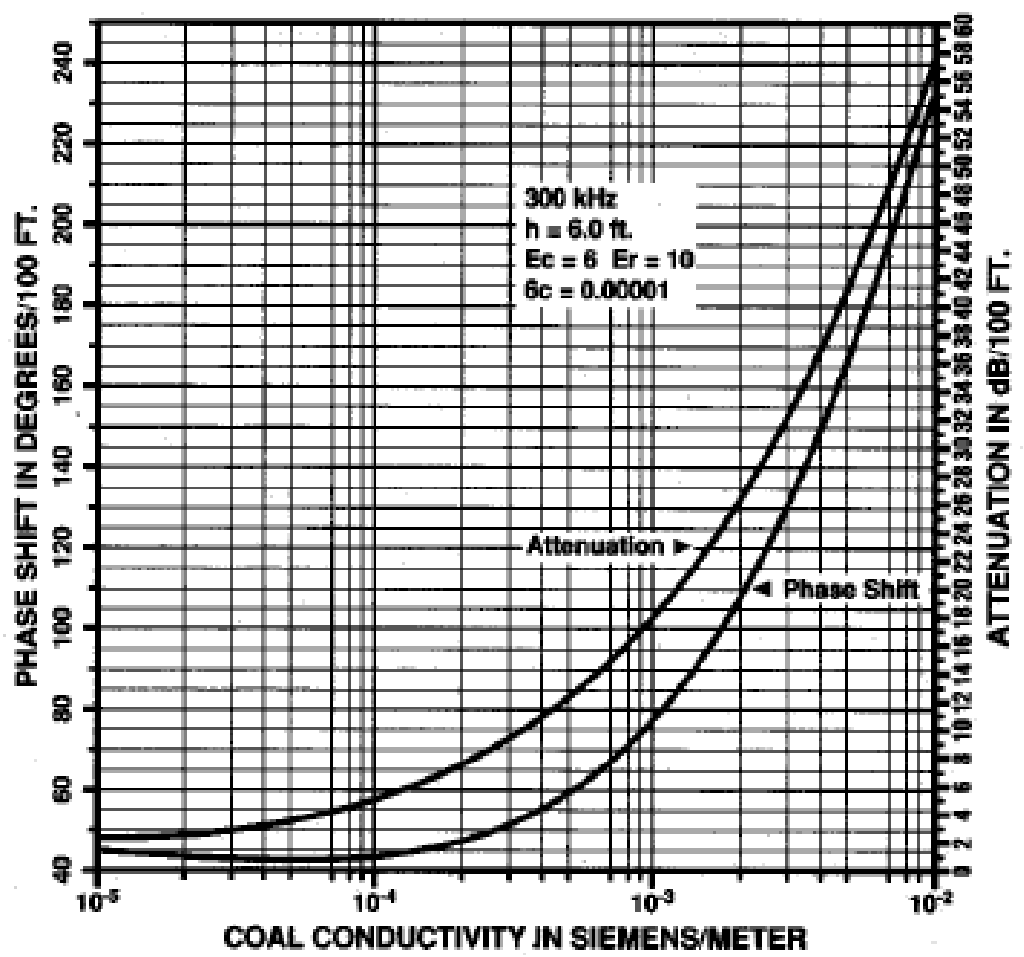

Figure 2.10 Attenuation rate/phase shift vs coal conductivity (Hill, 1984)

\subsection{RIM and Electromagnetic (EM) Seam Wave}

In the context of underground seam wave communications problems, Wait (1976) formulated the seam wave problems and determined the values of the propagation constants in a uniform seam. He was the first to recognize that natural waveguides exist in the earth's crust. From Wait's results, the seam wave would be highly responsive to changing seam conditions. Wait defined the coal seam wave propagation theory as illustrated in Figure 2.11 .

Radio wave energy propagation occurs in the seam because the higher conductivity sedimentary rock surrounding the seam force the radio wave energy to flow in the coal seam between the sediment layers. The electrical conductivity of shale, mudstone, and fire clay ranges from 0.01 to 0.1 Siemens per meter ( $\mathrm{S} / \mathrm{m})$ (100 and $10 \mathrm{ohm}$-meters). The conductivity of coal is near $0.0005 \mathrm{~S} / \mathrm{m}(2,000 \mathrm{ohm}$-meters). The 10-to-1 contrast in 
conductivity causes a waveguide to form and waves to travel within the coal seam. A smaller amount of energy escapes through imperfectly conducting sediment layers. Energy is also dissipated as heat in the seam layer. Inside the coal seam, $H_{y}$ and $E_{z}$ are the dominant field components, and they are nearly constant in z. The electric (E) field component of the EM wave is polarized between the mudstone and claystone sediment layers. The magnetic (Hy) field component is directed into the page. The horizontal electric field is zero at the center of the seam and is fairly small throughout the seam. Because the seam height is small as compared to the wavelengths, only cylindrically spreading zero order mode Transverse Electromagnetic (TEM) wave propagate in the wave guide. Higher order modes quickly vanish with distance from the radiating antenna. This means that the magnitude of the seam wave is constant across the height of the waveguide. If higher order modes could exist in the waveguide, the magnitude of the fields would vary with seam height (Stolarczyk and Fry, 1990).

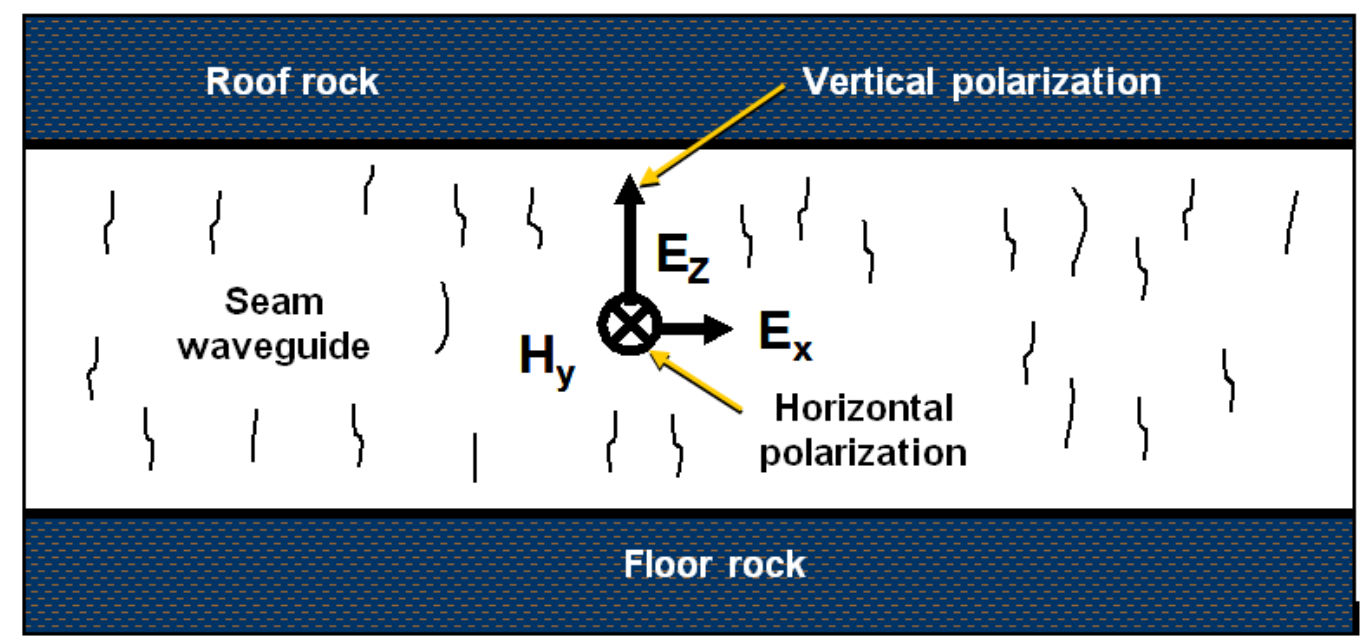

Figure 2.11 Natural waveguide for electromagnetic wave transmission (Stolar Horizon, Inc, 2004) 
Due to this waveguide behavior, the magnitude of the coal seam radio wave decreases with distance of travel because of two different factors, the attenuation rate and cylindrical spreading of wave energy in the coal seam. The cylindrically spreading factor is mathematically given by $1 / \sqrt{r}$, where $r$ is the distance from the transmitting to the receiving antenna. Comparing with the non-waveguide far-field spherically spreading factor of $1 / r$, the magnitude of the EM wave within the coal seam decreases by a factor of only 10 in the waveguide and by a factor of 100 in an unbounded media at 100 meters. An advantage of the seam waveguide is greater travel distance. Another advantage is that the traveling EM wave predominantly remains within the coal seam waveguide (Stolarczyk and Peng, 2003).

DeLonge (1982) determined the seam wave attenuation rate for a few seams and boundary layer conditions. Hill (1984) formulated the seam wave problems and investigated tomographic inversion of the measured coal seam data. Hill provided families of curves that related the seam wave propagation constants to the geologic electrical parameters of the seam (seam height and the electrical conductivity of the coal and the rock). The strong dependence of propagation constant on the thickness and conductivity of the sediment layers resolved the sensitivity issue. Hill (1986) and Shop et al., (1986) consider the theoretical problem of wave propagation in a uniform seam.

\subsection{RIM Tomography Method}

Tomography is a mathematical method of reconstructing the interior structure of a region from a finite number of projections through that region. The purpose of RIM tomography is reconstructing an attenuation rate tomogram to represents the geological characteristics in 
the panel of investigation. Shop (1987), Hill (1984), and Zhao (2005) analyzed the RIM tomography method. The following is a review of their research.

RIM transmission surveys are conducted between gateroads of a longwall panel as illustrated in Figure 2.12. A logical measurement scheme is used to step the transmitting and receiving loop antennas along the entries as shown in Figure 2.13. From the study of the EM seam wave (Section 2.2), this study assumes that no refraction takes place within the coal seam waveguide, and the propagation of a seam wave from a source station to a receiving station will follow a straight ray path. Usually, the number of transmitting and receiving stations is equal, and the stations are identically spaced. A uniform station layout provides the best ray path coverage of the panel interior. For example, if there are 10 transmitting and 10 receiving positions, then there are $10^{2}$ transmission measurements ray paths (Figure 2.13). The ray path from transmitting station one to receiving station one is labeled as ray number one, from transmitting station one to receiving station two is ray number to, etc. The coal panel is divided into a grid of $10 \times 10$, and the cells within the panel are labeled with a single index shown in Figure 2.13.

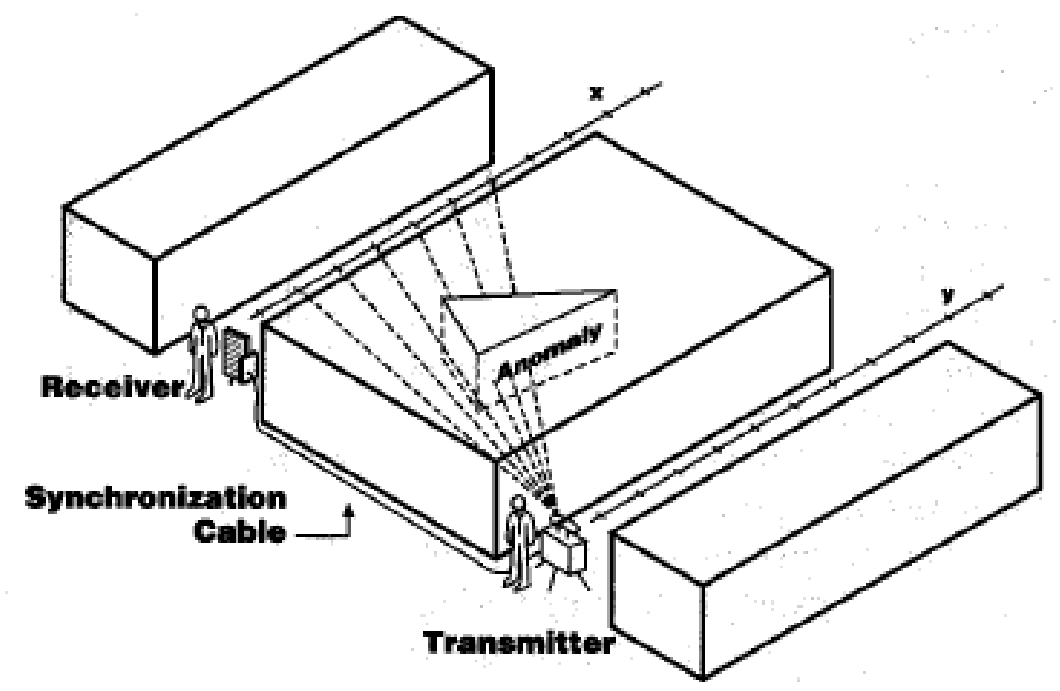

Figure 2.12 Radio wave signal paths in a coal seam (Stolarczyk and Fry, 1990) 


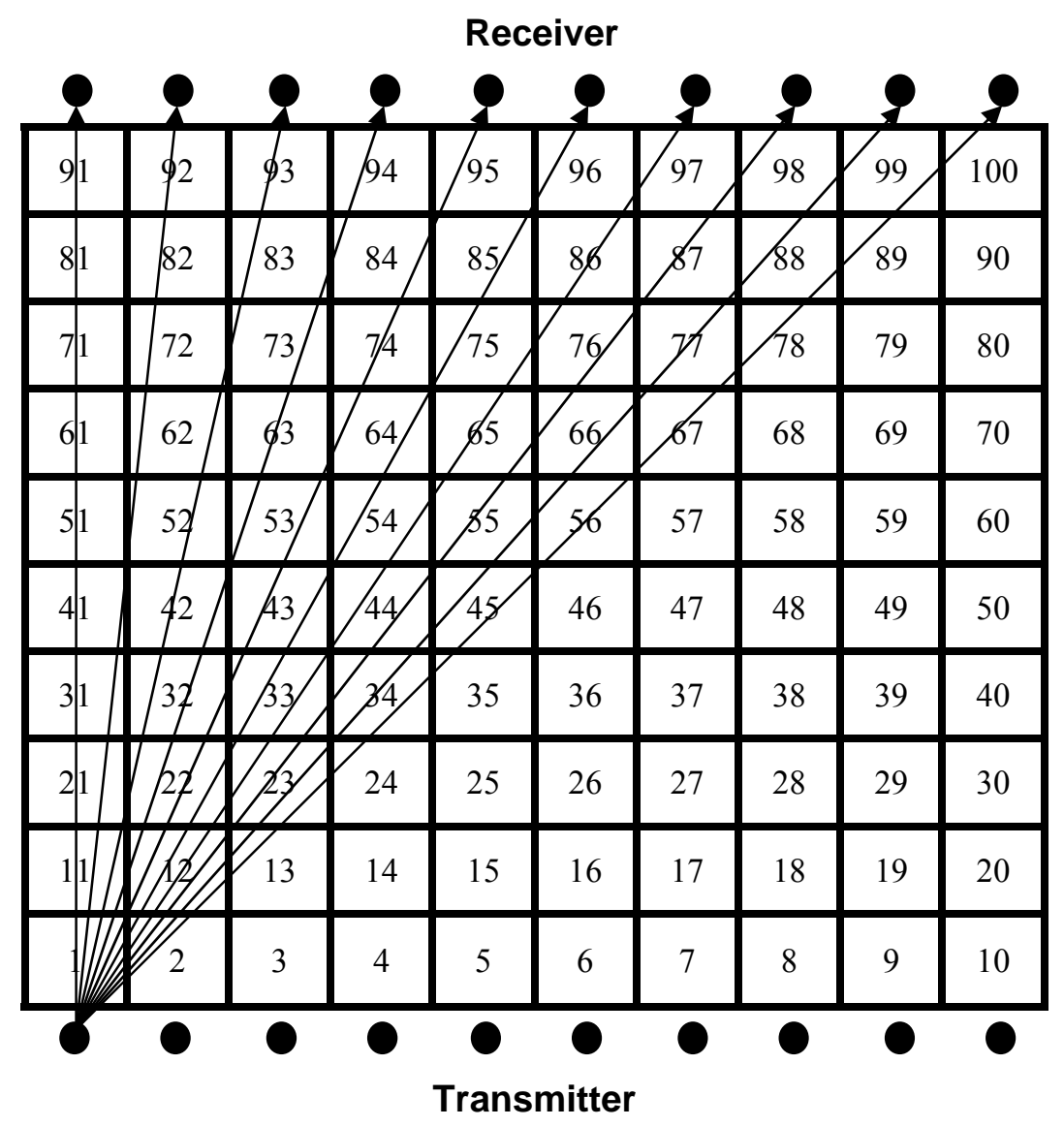

Figure 2.13 Ray path and cell index of RIM test

Based on the ray path and gridding scheme, the total attenuation of the signal, excluding the geometrical spreading loss, can be expressed as a straight-line integral along the ray path:

$$
\text { Total attenuation }=\int_{0}^{r} \alpha(r) d r
$$

where $r$ is the ray path distance between the transmitter and the receiver. $\alpha$ is the attenuation rate.

This one ray path total attenuation can be expressed as the summation of the ray path in each cell, 


$$
r_{11} \alpha_{1}+r_{12} \alpha_{2}+\ldots+r_{1 N} \alpha_{N}=Y_{1}
$$

where $r_{11}$ is the distance of the first ray path in cell $1, r_{12}$ is the distance of the first ray path in cell 2 , etc. $\alpha_{1}$ is the attenuation rate in cell $1, \alpha_{2}$ is the attenuation rate in cell 2 , etc. $Y_{1}$ is the total attenuation of the first ray path.

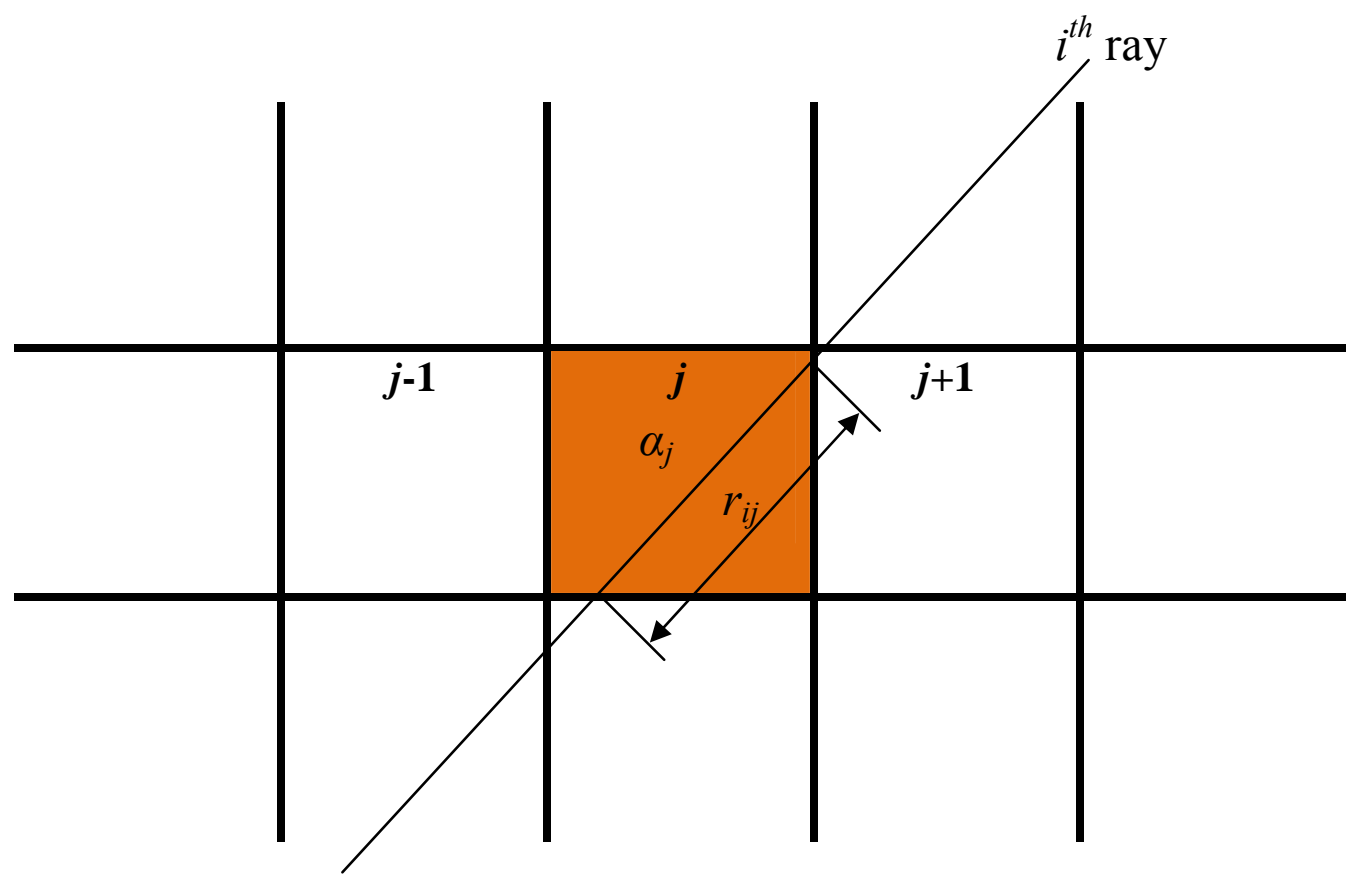

Figure 2.14 Geometry for the $i^{\text {th }}$ ray. $\alpha_{j}$ is the attenuation rate in the $j^{\text {th }}$ cell, and $r_{i j}$ is the length of the $i^{\text {th }}$ ray through the $j^{\text {th }}$ cell.

If there are $M$ rays path and $N$ cells, the total attenuation can be expressed as following $M$ equations,

$$
\begin{aligned}
& r_{11} \alpha_{1}+r_{12} \alpha_{2}+\ldots+r_{1 N} \alpha_{N}=Y_{1} \\
& r_{21} \alpha_{1}+r_{22} \alpha_{2}+\ldots+r_{2 N} \alpha_{N}=Y_{2} \\
& \ldots \\
& r_{M 1} \alpha_{1}+r_{M 2} \alpha_{2}+\ldots r_{M N} \alpha_{N}=Y_{M}
\end{aligned}
$$

or 


$$
\sum_{j}^{N} r_{i j} \alpha_{j}=Y_{i} \quad i=1,2,3, \ldots, M
$$

In matrix notation, this set of equations can be expressed as:

$$
\left[D_{i j}\right]\left[X_{j}\right]=\left[Y_{i}\right]
$$

where $D$ is the ray segment or geometry matrix, $X$ is the cell attenuation rate vector, and $Y$ is the measured net attenuation vector.

In theory, $\left[X_{j}\right]$ could be determined by matrix inversion or pseudo-inversion:

$$
\left[X_{j}\right]=\left[D_{i j}\right]^{-1}\left[Y_{i}\right]
$$

In practice, the inversion of the linear equation in Eq. (2-8) has several difficulties. The system of equations in Eq. (2-6) may be very large. For example, if the antenna spacing is $5 \mathrm{~m}$ over a length of $250 \mathrm{~m}$, then the number of equation is $50 \times 50=2500$. So if both of $M$ and $N$ are large, it is inaccurate to use conventional matrix theory methods to invert the system of Eq. (2-9). In spite of these difficulties, there are numerous techniques which have been successfully applied to geoscale tomogram problems (Lager et al., 1977; Dines et al., 1979; Radcliff et al., 1979). Some of the popular methods and their acronyms are: Algebraic Reconstruction Technique (ART) (Gordon, 1974), Simultaneous Iterative Reconstruction Technique (SIRT) (Gilbert, 1972), and Sparse Equations and Least Squares (LSQR) (Paige, 1982). These methods are based on the "method of projections" as first proposed by Kaczmarz (1937), and later elucidated further by Tanabe (1971).

To explain the computational steps involved in these methods, First, Eq. (2-8) is expanded as, 


$$
\begin{aligned}
& D_{11} X_{1}+D_{12} X_{2}+\ldots+D_{1 N} X_{N}=Y_{1} \\
& D_{21} X_{1}+D_{22} X_{2}+\ldots+D_{2 N} X_{N}=Y_{2} \\
& \ldots \\
& D_{M 1} X_{1}+D_{M 2} X_{2}+\ldots+D_{M N} X_{N}=Y_{M}
\end{aligned}
$$

A grid representation with $N$ cells gives image $N$ degrees of freedom. Therefore, an image, represented by $\left(X_{1}, X_{2}, X_{3}, \ldots, X_{N}\right)$, may be considered to be a single point in an $M$-dimensional space. In this space, each of the above equation represents a hyperplane. When a unique solution to these equations exists, the intersection of all these byperplanes is a single point giving that solution. This concept is further illustrated in Figure 2.15 where, for the purpose of display, only two unknowns $X_{1}$ and $X_{2}$ satisfying the equations (2-11) and (2-12) are shown,

$$
\begin{aligned}
& D_{11} X_{1}+D_{12} X_{2}=Y_{1} \\
& D_{21} X_{1}+D_{22} X_{2}=Y_{2}
\end{aligned}
$$

The calculation procedure begins with the initial guesses of values of $X_{1}^{0}$ and $X_{2}^{0}$, then these two values are projected on the first line represented by Eq. (2-11) to obtain $X_{1}^{1}$ and $X_{2}^{1}$. Then $X_{1}^{1}$ and $X_{2}^{1}$.are reprojected on the second line represented by Eq. (2-12) to obtain $X_{1}^{2}$ and $X_{2}^{2}$. The calculation of $X_{1}^{i}$ and $X_{2}^{i}$ are expressed by equations (2-13) and (2-14). This process is called iteration one.

$$
\begin{aligned}
& X_{1}^{i}=X_{1}^{i-1}+\frac{Y_{i}-y_{i}}{D_{11}^{2}+D_{12}^{2}} D_{i 1} \\
& X_{2}^{i}=X_{2}^{i-1}+\frac{Y_{i}-y_{i}}{D_{11}^{2}+D_{12}^{2}} D_{i 2} \quad(\mathrm{i}=1,2)
\end{aligned}
$$

where 


$$
y_{i}=X_{1}^{i-1} D_{i 1}+X_{2}^{i-1} D_{i 2}
$$

In the next iteration, $X_{1}^{2}$ and $X_{2}^{2}$.are projected back on the first line and then on the second line and so on, until the final solution $X_{1}^{\text {final }}$ and $X_{2}^{\text {final }}$ are achieved based on the pre-set number of iterations or pre-set tolerance. This iterative process $\left(X_{1}^{0}, X_{2}^{0}\right) \rightarrow\left(X_{1}^{1}, X_{2}^{1}\right) \rightarrow\left(X_{1}^{2}, X_{2}^{2}\right) \rightarrow \ldots \rightarrow\left(X_{1}^{\text {final }}, X_{2}^{\text {final }}\right)$ is called "Method of Projections" which is illustrated in Figure 2.15.

For solving all the unknowns $(\mathrm{N})$ in the equations (2-10), the same method as described the above is used. First, the initial guesses $\left(X_{1}^{0}, X_{2}^{0}, X_{3}^{0}, \ldots, X_{N}^{0}\right)$ are projected on the first equation in (2-10), then to the second equation in (2-10), and so on. When the $i$ th equation in (2-10) is taken up, the values for the $j$ th cell is calculated as

$$
X_{j}^{i}=X_{j}^{i-1}+\frac{Y_{i}-y_{i}}{\sum_{j=1}^{N} D_{i j}^{2}} D_{i j}
$$

The change value for the $j$ th cell due to the $i$ th equation in $(2-10)$ is

$$
\Delta X_{j}^{i}=X_{j}^{i}-X_{j}^{i-1}=\frac{Y_{i}-y_{i}}{\sum_{j=1}^{N} D_{i j}^{2}} D_{i j}
$$

where

$$
y_{i}=\sum_{j=1}^{N} D_{j}^{i-1} D_{i j}
$$

Once the change value for each cell has been achieved, it can be put in the cell to obtain the new value for the cell. Finally, the unknown values in equations (2-7) can be solved (Zhao, 2005). 


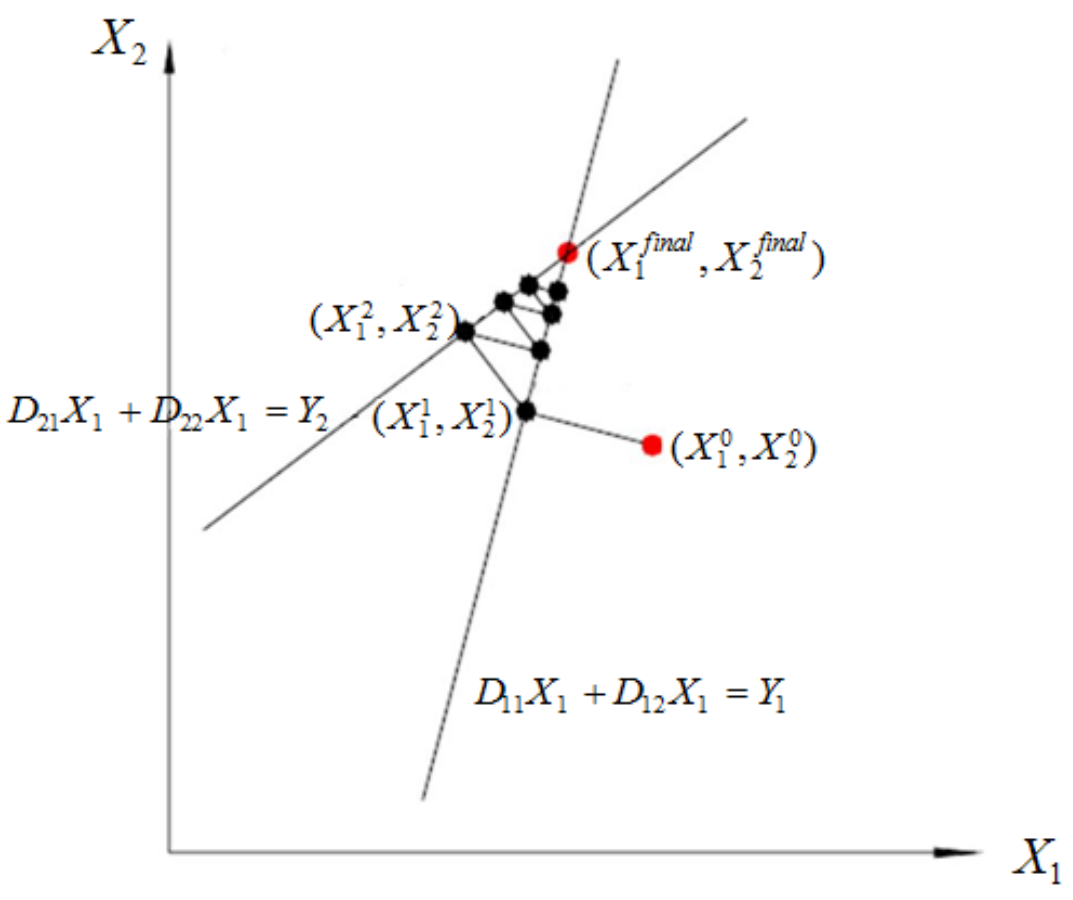

Figure 2.15 Method of projection (Zhao, 2005).

\section{Algebraic Reconstruction Technique (ART)}

For this algorithm, Eq. (2-16) is used to calculate the change value for the $j$ th cell due to the $i$ th equation in (2-10), at the same time the change value is put in the $j$ th cell. Since the value of each cell is changed when the new equation is being solved, the solution convergence for this approach is fast, but the reconstructed image may not be good (Kak and Slaney, 1999).

\section{Simultaneous Iterative Reconstruction Technique (SIRT)}

For this algorithm, Eq. (2-16) is still used to calculate the change value for the $j$ th cell due to the $i$ th equation in (2-10), but the value for the $j$ th cell is not changed at this time. Before making any change, all the equations in (2-10) are processed. After all the equations in $(2-10)$ are taken up, the change value for the $j$ th cell is calculated by averaging all the calculated changes for that cell, and then put this value to the $j$ th cell. This process only needs one iteration and then goes back to the first Eq. (2-10) to begin the next iteration. The 
process is repeated. Compared to ART technique, the solution convergence is slower but the final image is smoother. In all, since each the equation in (2-10) represents one ray path, ART technology changes the cell values found in processing each ray path data. Conversely, SIRT technology changes the cell values after processing all the ray paths (Kak and Slaney, 1999).

\subsection{EM Scale Model Research Method}

Scale modeling is widely used in the field of science to simulate or visualize natural phenomena which is difficult to represent analytically and/or numerically. Laboratory scale models provide different opportunities from computer simulations and analytical studies, and they can be relatively inexpensive and easy to implement (Peden et al., 1995). For example, modeled targets may be made three-dimensional as easily as two-dimensional and their cross-sections can assume a variety of configurations. The scaled longwall panel may be partially filled with dielectrics, i.e. water, or with conductors to make some artificial geological structure anomalies.

Sinclair (1948), King and Smith (1981), and King (1982) described the basic theory of scale models used in electromagnetic research. For a scale model to provide accurate information about a full-sized system, the physical parameters for the model and full-sized system must satisfy certain relationships. The following is a review of the theory.

Electromagnetic scale modeling is a powerful experimental tool for determining the electrical performance of a system from measurements on a model with physical lengths that differ from those of the full-sized system by a factor $k_{l}$. This factor may be less than or greater than one and is usually chosen to make the size of the model manageable in the laboratory. For the scale model to be an accurate simulation of the full-sized system, the 
parameters that describe it must satisfy definite relations determined from the electromagnetic boundary-value problem used here, or alternatively, from dimensional analysis.

For the "geometrical" model, the real physical dimensions $\left(r_{f}\right)$ are scaled by a factor $k_{l}$ to scale the dimension $r_{m}$ as shown in Eq. (2-18). Due to the fact that the radio wave length should be reduced by $k_{l}$ in dimension, the frequency $\left(\omega_{f}\right)$ is scaled by the factor $k_{\omega}=1 / k_{l}$ to a scaled model frequency of $\omega_{m}$.

$$
\begin{aligned}
& r_{m}=k_{l} r_{f} \\
& \omega_{m}=k_{\omega} \omega_{f}=\left(1 / k_{l}\right) \omega_{f}
\end{aligned}
$$

where the subscripts $f$ and $m$ refer to the full-sized system and the scaled model, respectively. The electrical constitutive parameters for every material in the full-sized system are also scaled. The scale factors are:

For the permittivity $k_{\varepsilon}=1$,

$$
\varepsilon_{m}\left(\omega_{m}\right)=k_{\varepsilon} \varepsilon_{f}\left(\omega_{f}\right)=\varepsilon_{f}\left(\omega_{f}\right)
$$

For the electrical permeability $k_{\mu}=1$,

$$
\mu_{m}\left(\omega_{m}\right)=k_{\mu} \mu_{f}\left(\omega_{f}\right)=\mu_{f}\left(\omega_{f}\right)
$$

For the electrical conductivity $k_{\sigma}=1 / k_{l}$,

$$
\sigma_{m}\left(\omega_{m}\right)=k_{\sigma} \sigma_{f}\left(\omega_{f}\right)=\left(1 / k_{l}\right) \sigma_{f}\left(\omega_{f}\right)
$$

From Eq. (2-20) the permittivities of the materials in the full-sized system and the model must be the same. The condition defined in Eq. (2-21) for the permeabilities is easily met when all materials in the full-sized system and model are nonmagnetic $\mu=\mu_{0}$. The 
conductivities specified by Eq. (2-22) for the full-sized system must be scaled by the factor $1 / k_{l}$ for the model.

Hyun et al. (2007) constructed a laboratory scaled-down model and measured the underground pipe leakage by Ground Penetrating Radar (GPR). The laboratory system consisted of a dry sand tank, a pipe, and a simulated zone of leakage adjacent to the pipe. The size and burial depth of the pipe were scaled down to about $1 / 6$ of the real world condition. An equivalent leakage model was employed using an acrylic plastic box filled with methanol. A support for the model was provided by acrylic plastic plates and tubes with dry sand filling. The electrical properties of the equivalent leakage and support were verified by measuring their complex permittivities. B-scan radar images were displayed by background removal and neighbouring difference of raw data. For three cases of leaky pipes, the images showed the superimposition of nearly symmetric and inverted hyperbolas produced by non-leaky pipes and the blurring effects caused by the leakage.

Some other electromagnetic scale model experiments for ground penetrating radar also have been performed (Smith and Scott, 1989, 1990; Peden and Brew, 1995; Bernabini et al., 1995), but none study the application of RIM technology to coal mines. 


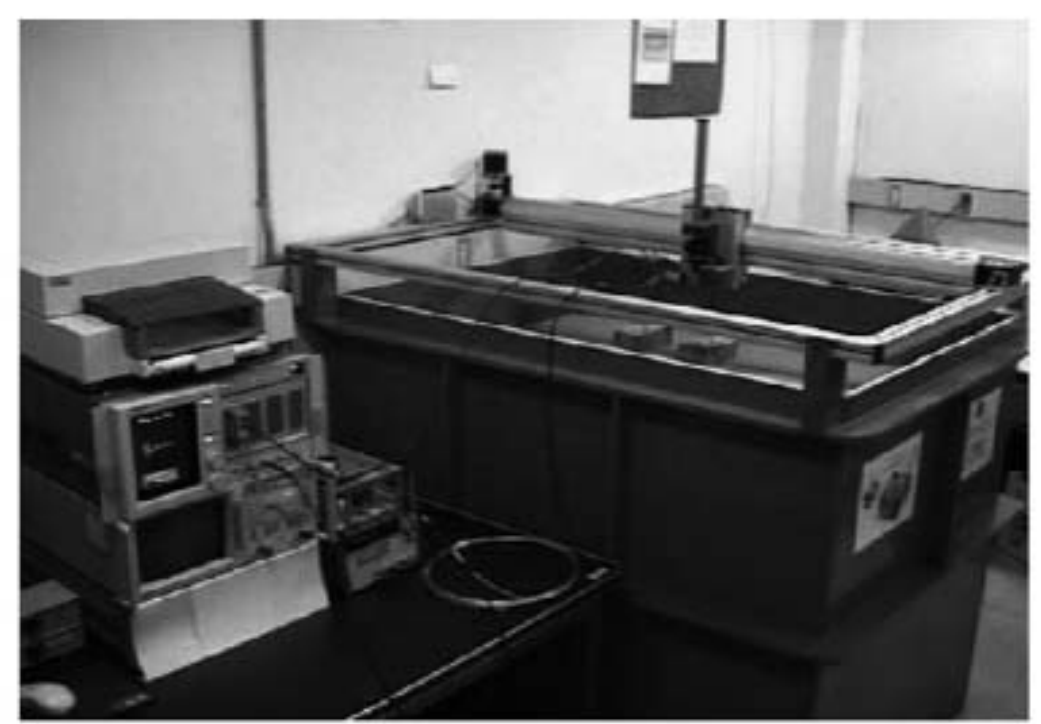

(a)

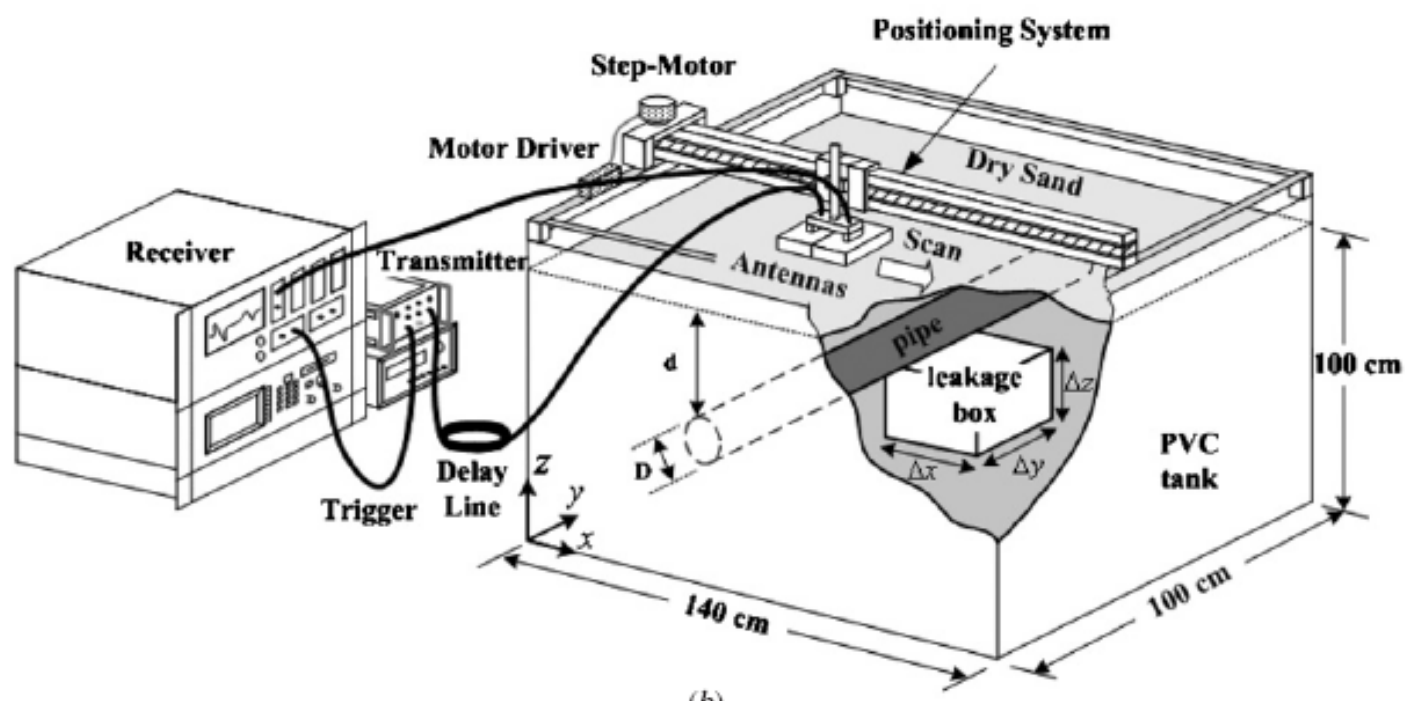

(b)

Figure 2.16 Laboratory scale GPR system. (a) Real system and (b) configuration 


\section{CHAPTER 3 EXPERIMENTAL PROGRAM}

\subsection{EM Properties Testing of Simulation Materials}

Sand (S), gypsum (G), cement (C), coal powder (Co), salt (SA), and water were selected to construct the scale model. Except salt, all the materials have been widely used in coal mine ground control to study the overburden movement and stress distribution (Peng et al., 1987; Qian, 1996, and Lin, 1984). Salt will be used to increase the electrical conductivity in the roof/floor, and the electrical conductivity difference between the roof/floor and the coal seam. Another advantage to using those materials is that they are abundant and inexpensive. In the scale model, sand, gypsum, cement, salt, and water will be used to build the roof and floor. Sand, coal powder, gypsum, and water will be used to build the coal seam.

In order to obtain the materials with proper EM properties (dielectric constant and electrical conductivity) to build the scale physical model, samples with different ratios of sand, gypsum, cement, salt, and water were made. These samples were compacted and the surface was made flat for testing. Some of these samples are shown in Figure 3.1.

The coaxial probe method for measuring EM properties was used to test the samples. The measurement system consists of a network or impedance analyzer, a coaxial probe, software, and a computer. In this study, a HP 8714ES network analyzer and a Agilengt $85070 \mathrm{E}$ dielectric probe kit were selected. Both the software (Agilent IO libraries software suite) and the probe are included in the $85070 \mathrm{E}$ dielectric probe kit. An external computer is needed to control the network analyzer through an Agilengt 82357A USB/GBIP interface which provides a convenient and flexible way to realize this connection (Figure 3.2). 


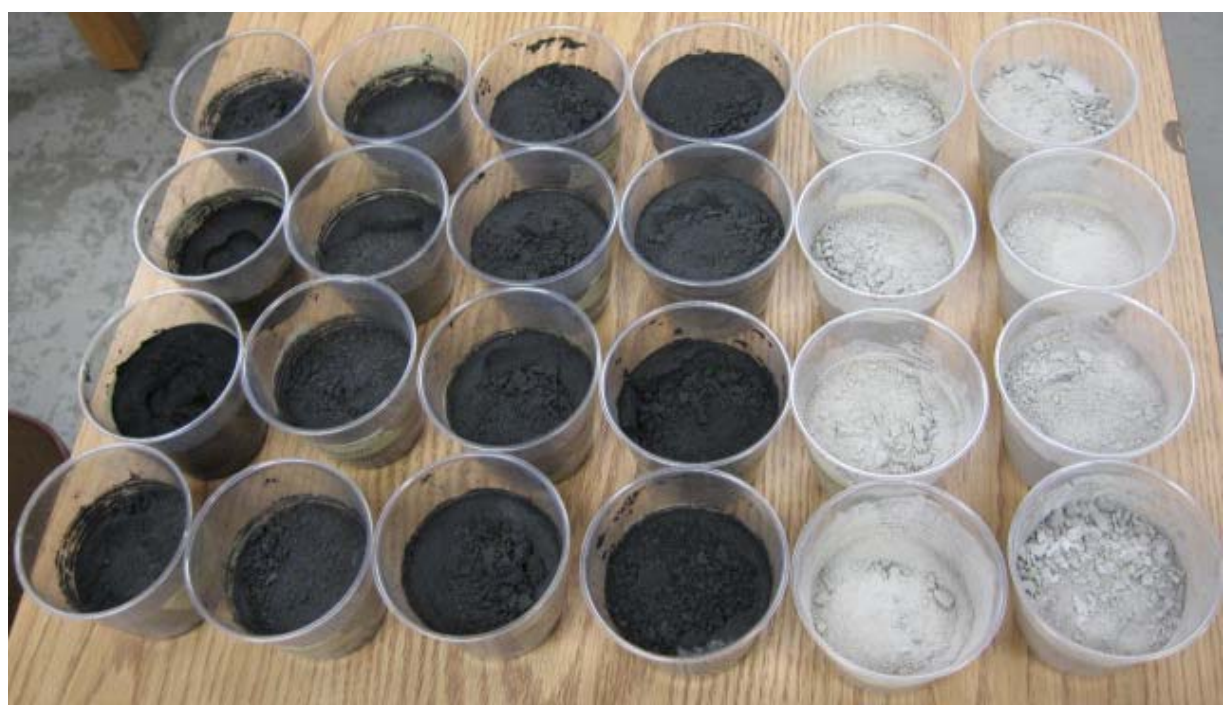

Figure 3.1 Part of the samples with different ratios of sand, gypsum, cement, salt, and water

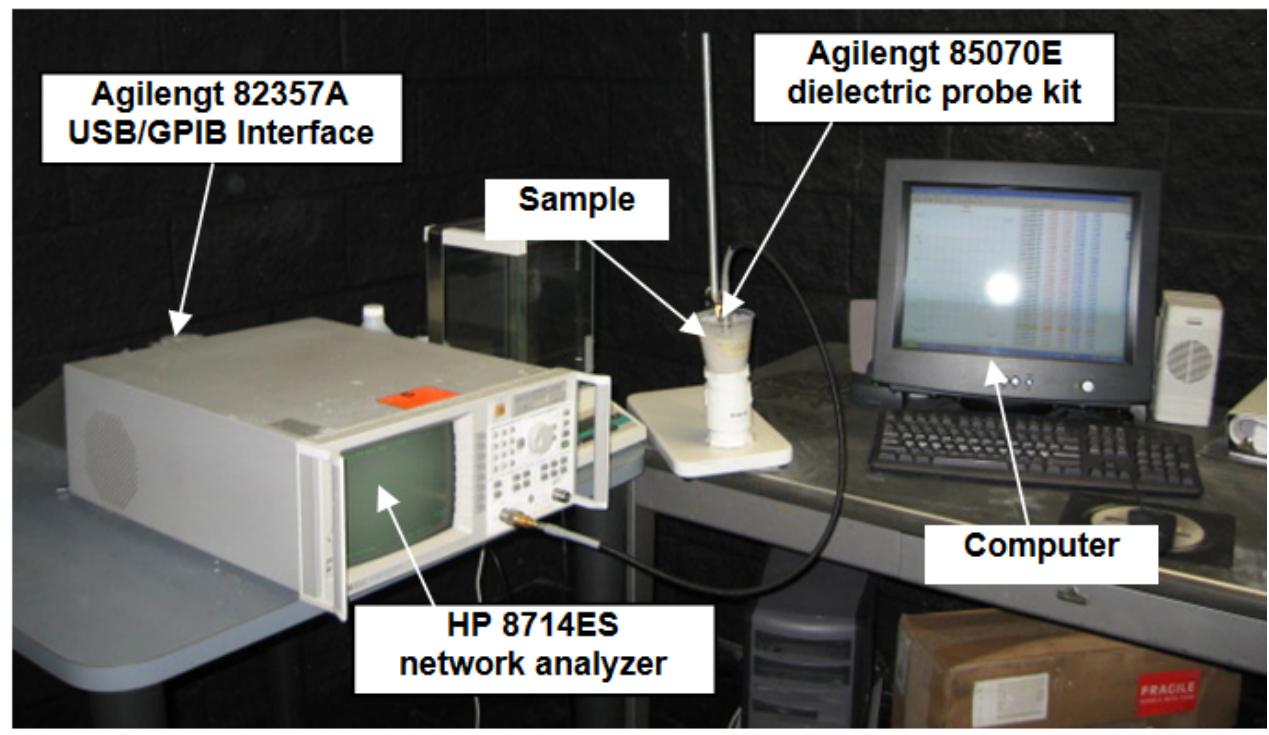

Figure 3.2 EM properties testing equipment

In the testing process, specimens of different ratios of component materials and water content were made and tested. Since high frequency antenna (900 MHz in this study) will be used in the laboratory test, it is necessary to measure the electrical properties at high frequency. The testing frequency range was set from $300 \mathrm{MHz}$ to $2 \mathrm{GHz}$. After all the samples have been tested, the dielectric constant at $900 \mathrm{MHz}$ was selected from the testing 
results and the electrical resistivity/conductivity was calculated consequently according to Eq. (2-2). All the data obtained from the network analyzer were normalized by exponential regression. Part of the samples with the mixture of $\mathrm{S}: \mathrm{Co}: \mathrm{C}=5: 5: 1, \mathrm{~S}: \mathrm{Co}: \mathrm{C}=6: 6: 1$, $\mathrm{S}: \mathrm{Co}: C=7: 7: 1, \mathrm{~S}: \mathrm{Co}: C=8: 8: 1, \mathrm{~S}: \mathrm{G}: C=5: 0.5: 0.5, \mathrm{~S}: \mathrm{G}: C=7: 0.5: 0.5, \mathrm{~S}: \mathrm{G}: \mathrm{C}: \mathrm{SA}=6: 0.5: 0.5: 0.05$, S:G:C:SA=6:0.5:0.5:0.1, S:G:C:SA=6:0.5:0.5:1, S:G:C:SA=7:0.5:0.5:05, and $\mathrm{S}: \mathrm{G}: \mathrm{C}: \mathrm{SA}=7: 0.5: 0.5: 1$ were shown and analyzed in this study.

Figures 3.3 and 3.4 show the testing results for roof/floor specimens with a mixture of S:G:C:SA=7:0.5:0.5:1 and S:G:C:=7:0.5:0.5. From the testing results, water content and salt have a large effect on the dielectric constant and electric conductivity. The dielectric constant increases with increasing water content. The electric conductivity will increase with increasing water content and salt content. The water will affect both dielectric constant and electrical conductivity, and the salt mainly affects the electric conductivity (Figure 3.4). From this figure, the dielectric constant of the specimens ( $\mathrm{S}: \mathrm{G}: \mathrm{C}: \mathrm{SA}=7: 0.5: 0.5: 1$ and S:G:C: $=7: 0.5: 0.5)$ increases with the increase of water content, and their values are very close to each other. In the same figure, the electric conductivity of specimen $\mathrm{S}: \mathrm{G}: \mathrm{C}=7: 0.5: 0.5$ does not change much with different salt contents. However, the electric conductivity of specimen $\mathrm{S}: \mathrm{G}: \mathrm{C}: \mathrm{SA}=7: 0.5: 0.5: 1$ changes considerably with different salt contents. Since in real condition, the dielectric constant and electric conductivity are larger than those of the coal seam, this characteristic is very useful in building the physical scale model. 


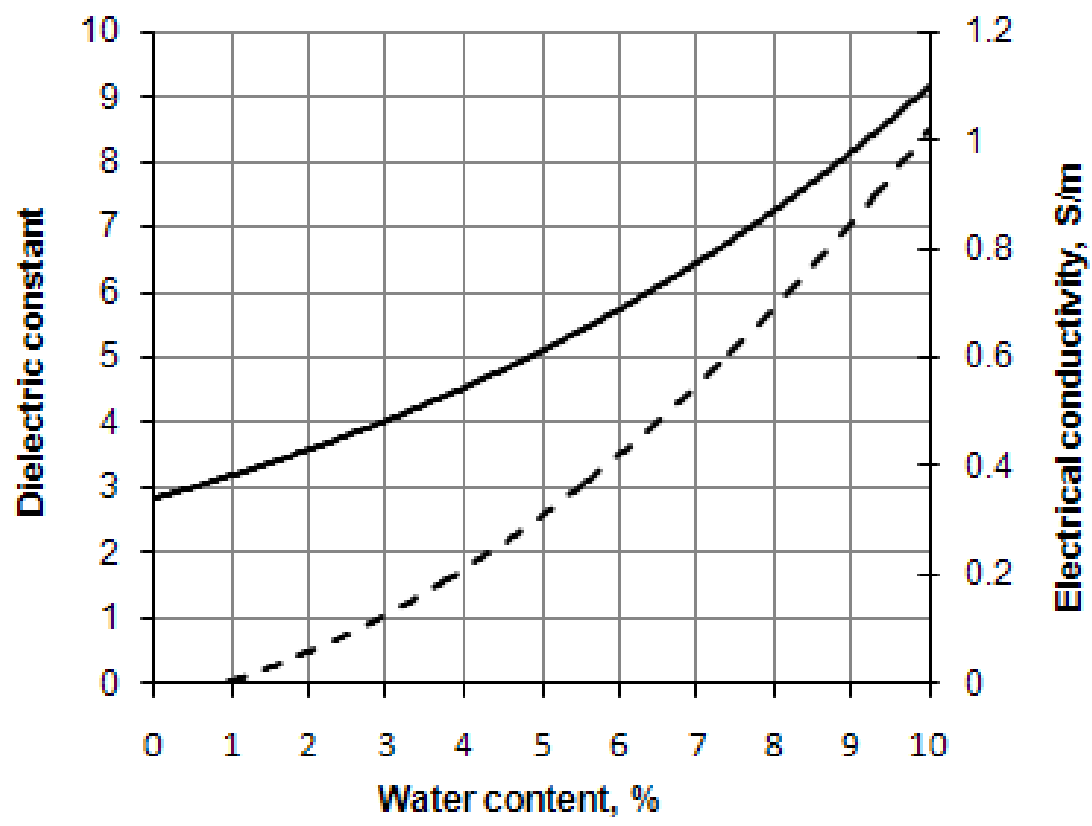

_ Dielectric constant -.--- Electrical conductivity

Figure 3.3 Water content effect on dielectric constant and electrical conductivity (S:G:C:SA=7:0.5:0.5:1)

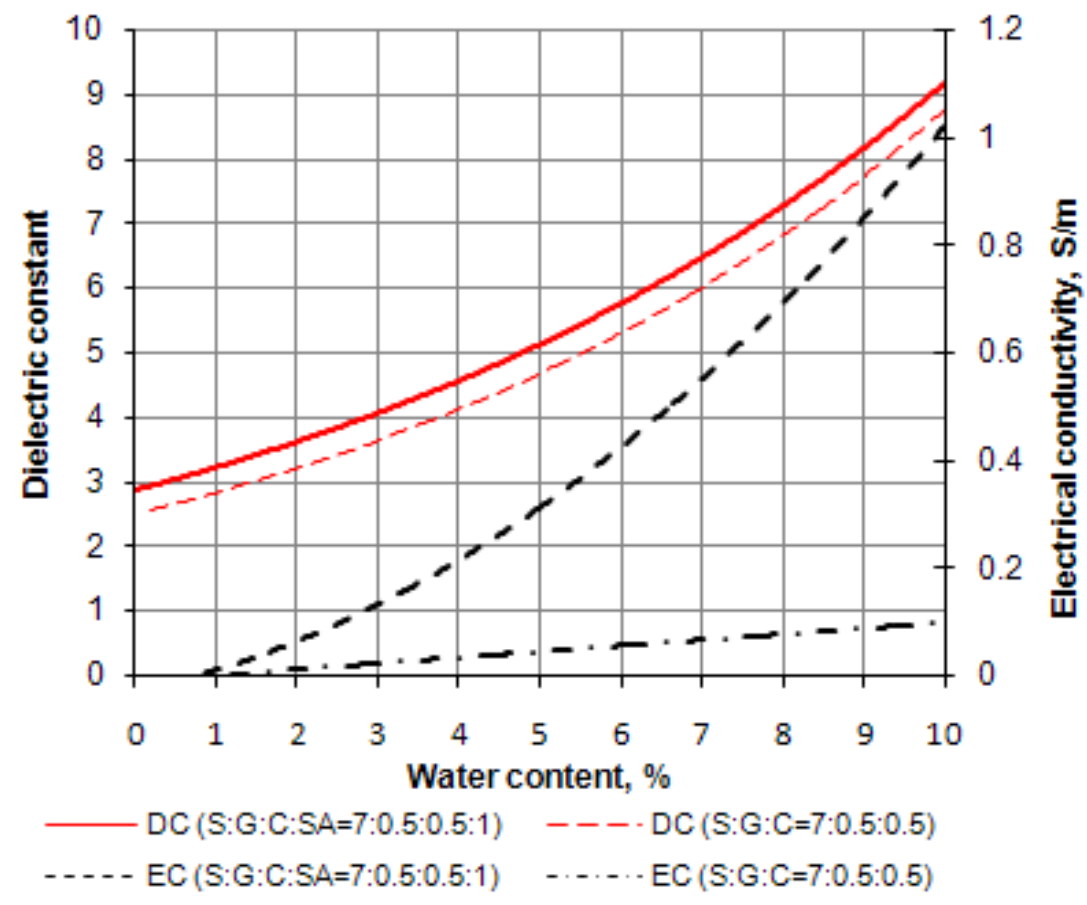

Figure 3.4 Water content and salt content effect on dielectric constant and electrical conductivity 
Figure 3.5 shows the dielectric constant at different components mixture ratios and different water contents to simulate the roof/floor. Except sand, cement, and gypsum, salt has been added to the samples to increase the electrical conductivity for the roof/floor. For certain water content, the dielectric constant values are really close to each other. The maximum values at $10 \%$ of water content range from 8 to 9.5 and the minimum values at $0 \%$ of water content range from 1.5 to 3 .

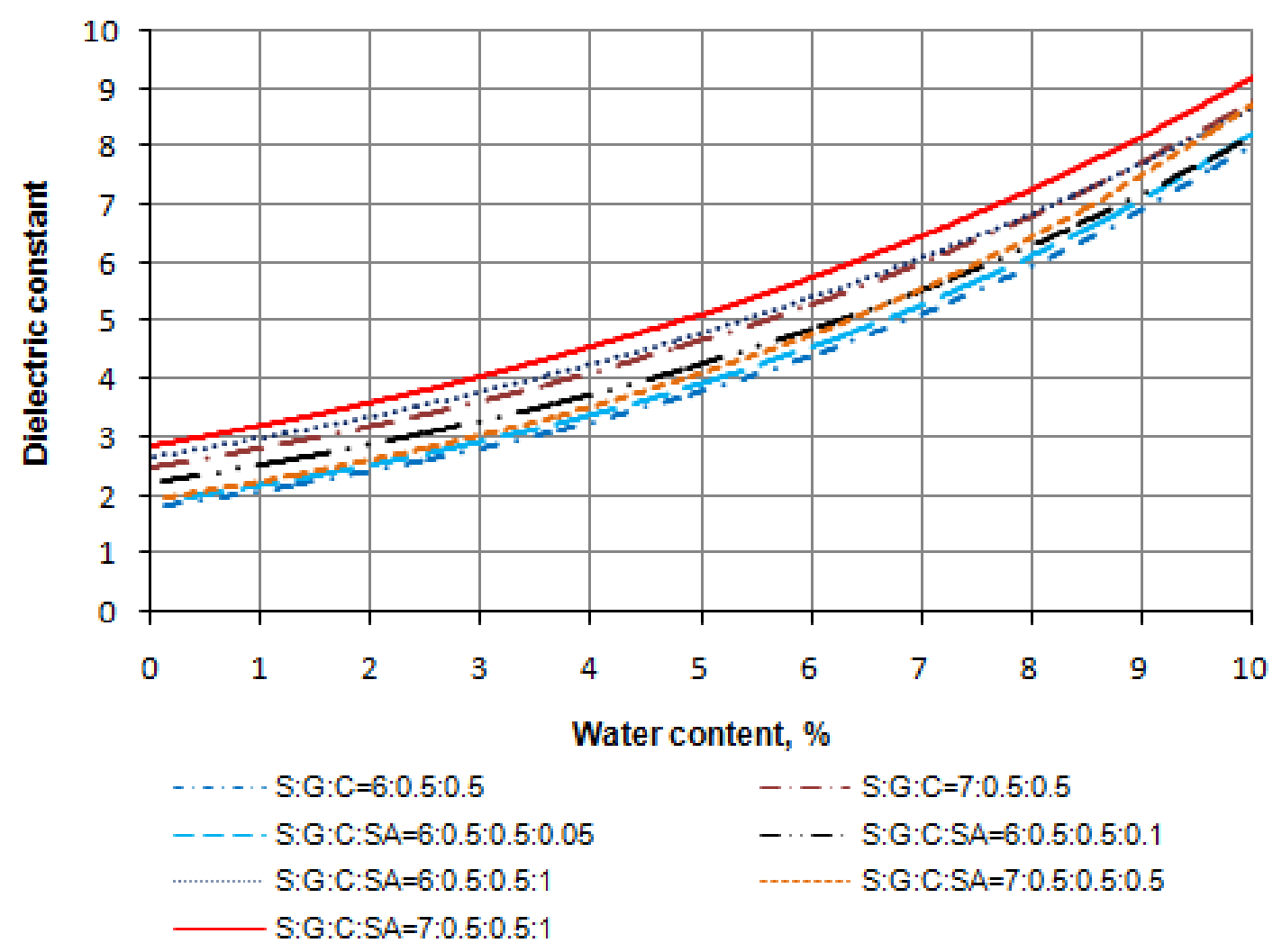

Figure 3.5 Dielectric constant at different components mixture ratios and different water content to simulate the roof/floor

Figure 3.6 shows the electrical conductivity at different components mixture ratios and different water contents to simulate the roof/floor. For certain water content, the electrical conductivity values vary considerably. The maximum values at $10 \%$ of water 
content range from $0.1 \mathrm{~S} / \mathrm{m}$ to $1.5 \mathrm{~S} / \mathrm{m}$ and the minimum values at $0 \%$ of water content are really close to each other. These characteristics also verify the effect of water content and salt content on the electrical conductivity.
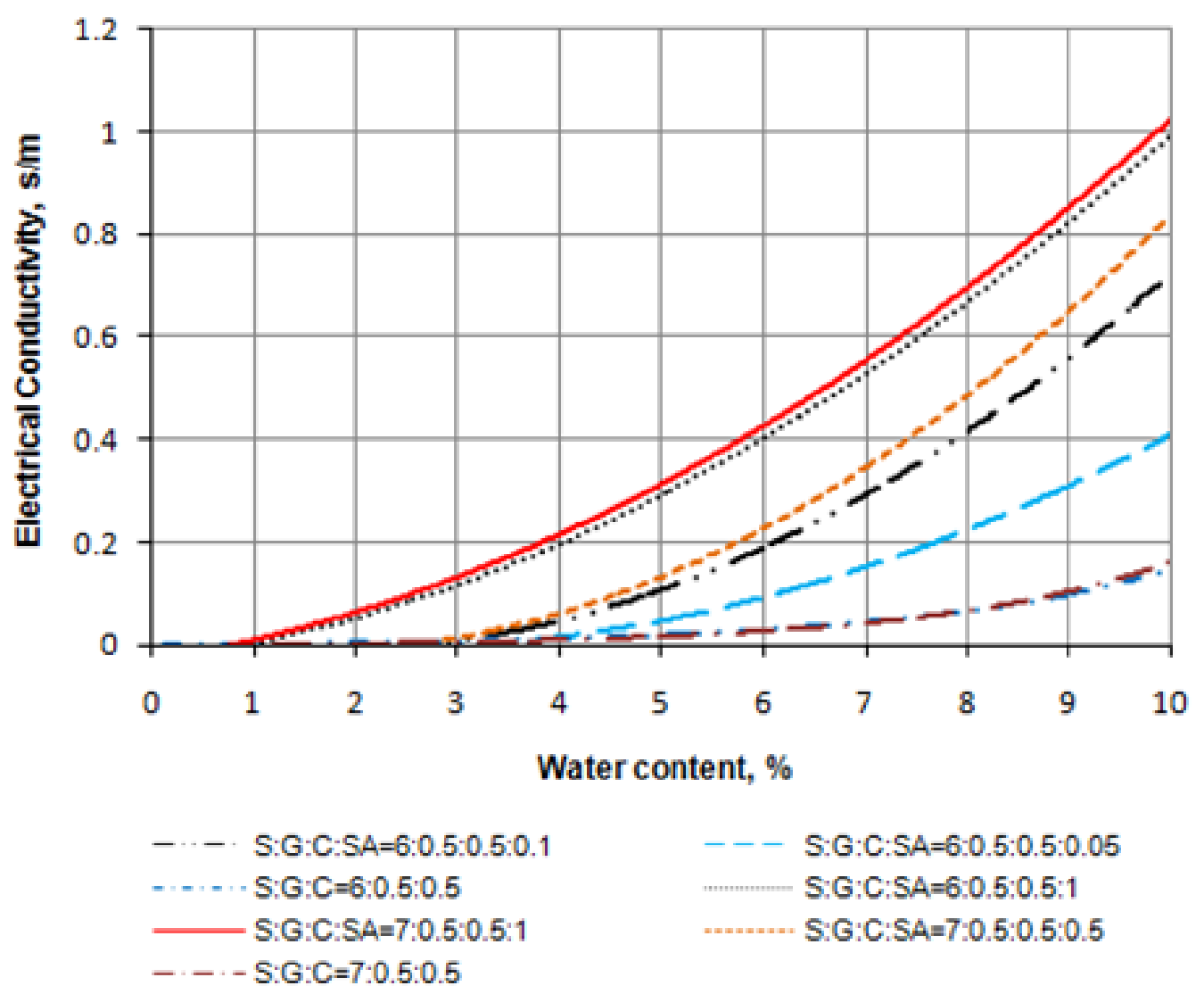

Figure 3.6 Electrical conductivity at different components mixture ratios and different water content to simulate the roof/floor

Figure 3.7 shows the dielectric constant at different components mixture ratios and different water contents to simulate the coal seam. In these samples, no salt was added to keep the electrical conductivity low. For certain water content, the dielectric constant values are also close to each other. The maximum values at $10 \%$ water content range from 4 to 5 and the minimum values at $0 \%$ of water content range from 2 to 3 . 


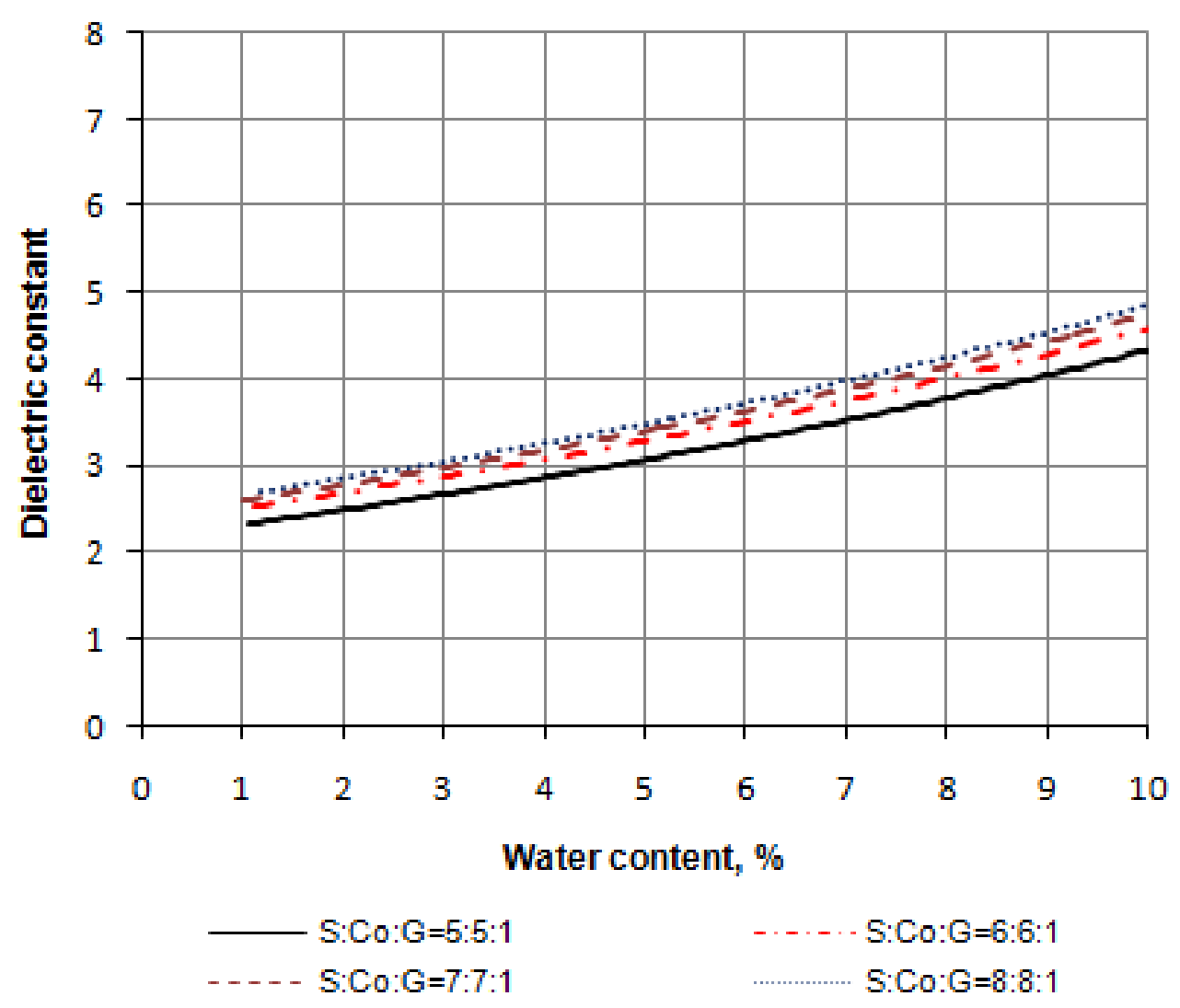

Figure 3.7 Dielectric constant at different components mixture ratios and different water content to simulate the coal seam

Figure 3.8 shows the electrical conductivity at different components mixture ratios and different water contents to simulate the coal seam. Since there is no salt in the samples, for certain water content, the electrical conductivity values exhibit only minor difference. The maximum values at $10 \%$ of water content range from $0.01 \mathrm{~S} / \mathrm{m}$ to $0.015 \mathrm{~S} / \mathrm{m}$ and the minimum values at $1 \%$ of water content range from $0 \mathrm{~S} / \mathrm{m}$ to $0.005 \mathrm{~S} / \mathrm{m}$. 


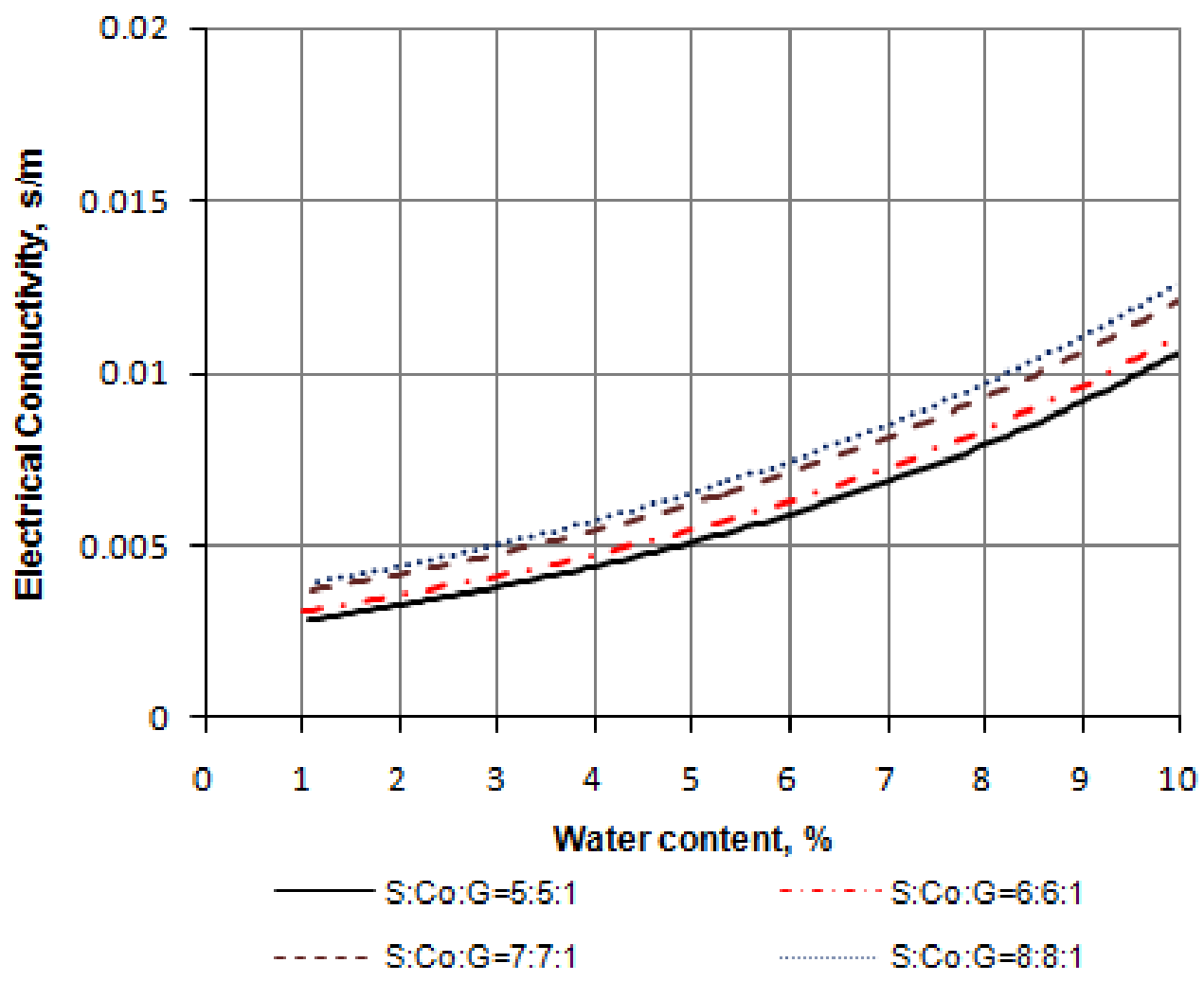

Figure 3.8 Electrical conductivity at different components mixture ratios and different water content to simulate the coal seam

\subsection{Laboratory Physical Model Testing Equipment}

Geophysical Survey System, Inc. (GSSI) Subsurface Interface RADAR (SIR)® II control unit with two $900 \mathrm{MHz}$ antennas (Figure 3.9) was selected to test the model. There are both transmitter and receiver in one antenna, but it is not necessary in this study. In order to meet the requirement for this study, the antenna with the receiver part disabled will be used as the transmitter; and the antenna with the transmitter part disabled will be used as the receiver. Since the transmitting antenna and receiving antenna will be separated and put in opposite sides of the physical model, a break-out box has been selected to separate the transmitting antenna and receiving antenna. 


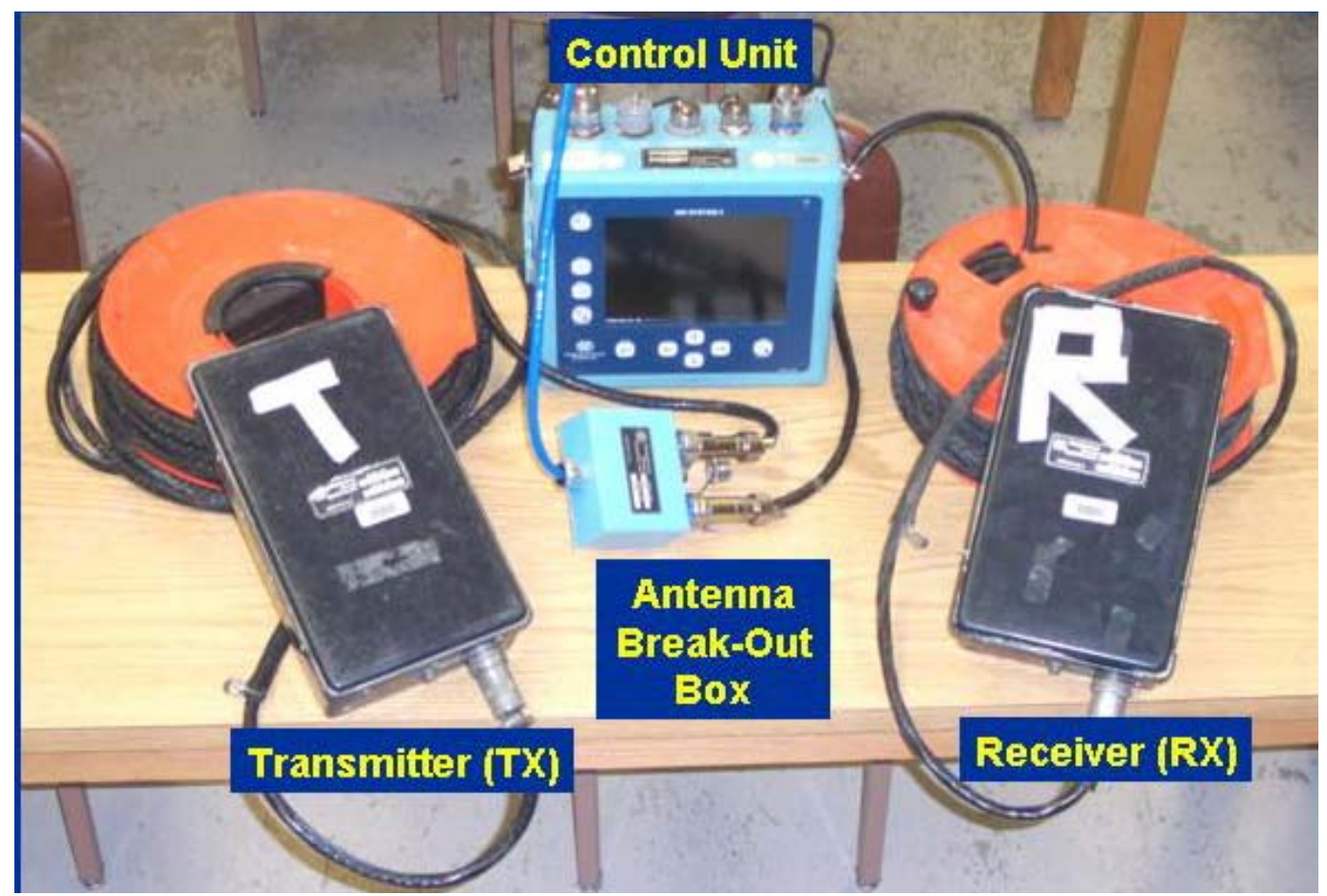

Figure 3.9 SIR II and radars

The layout of the scale model testing system is shown in Figure 3.10.

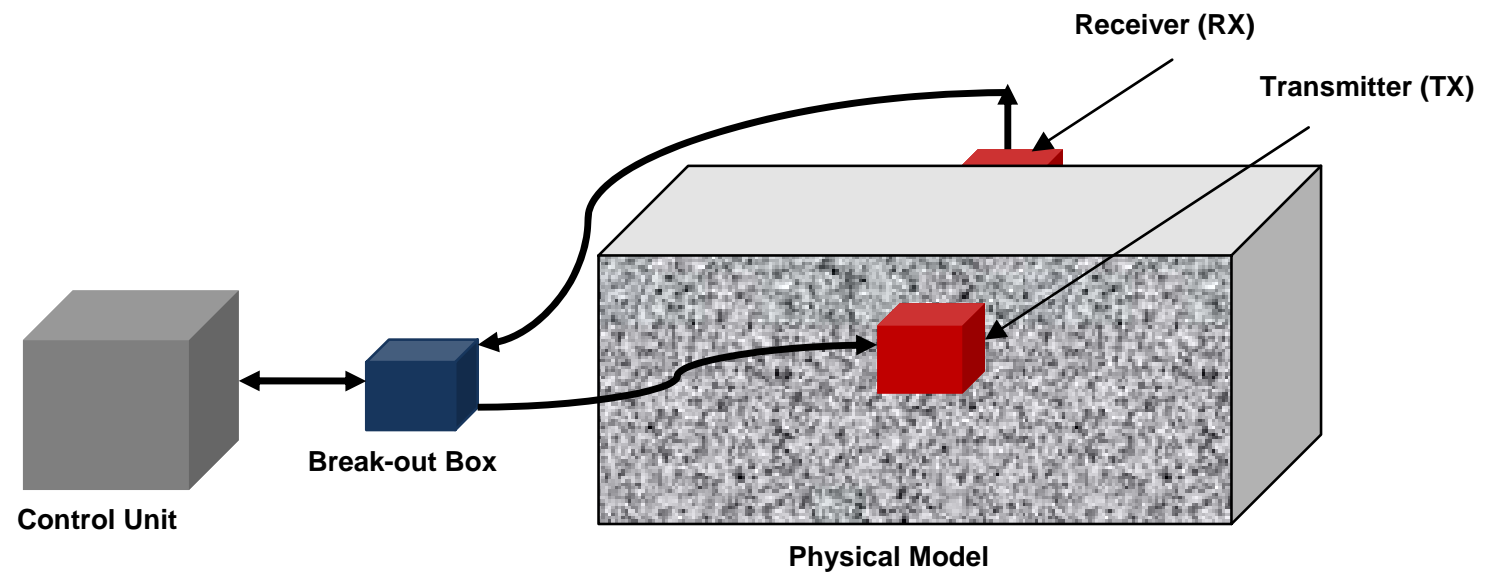

Figure 3.10 Scale model testing system layout

Since water content has a large effect on the EM properties of the materials and the RIM testing result, it is important to monitor the water content in the physical model during 
construction and in the testing process. A moisture meter has been selected to test the water content (Figure 3. 11).

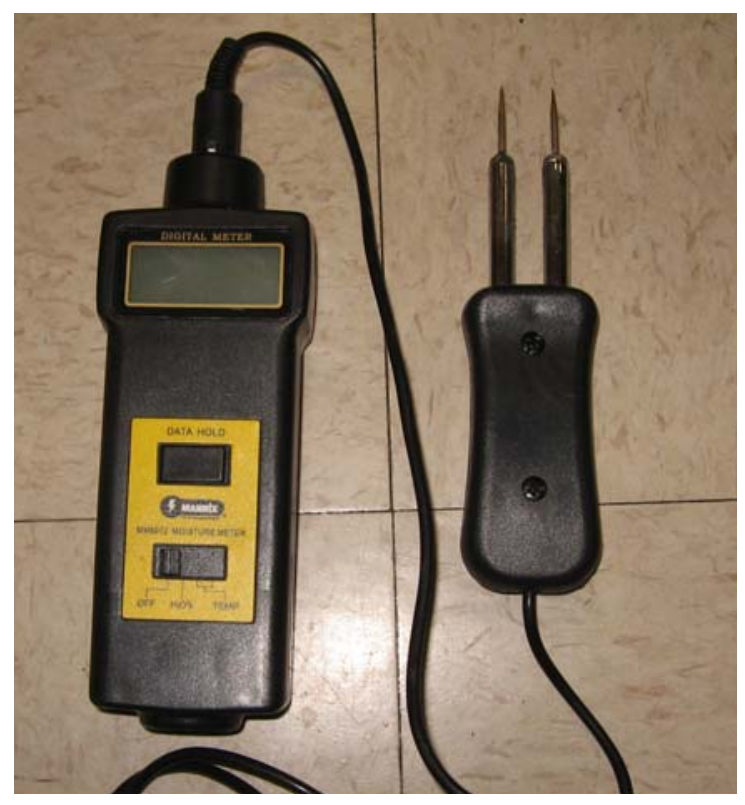

Figure 3.11 Moisture meter

\subsection{Physical Model Testing Procedure}

The process of collecting tomogram data sets involves a series of measurement including calibration testing, reconnaissance testing and RIM testing.

\subsubsection{Calibration Testing Method}

A RIM calibration test should be conducted prior to the tomogram survey operation. The calibration test provides characteristic site parameters vital in the RIM tomogram data analysis, including the Standard Attenuation Rate (SAR) and Transmitter Coupling Factor (C-Factor). These parameters are unique to the electrical and stratigraphic characteristics of the specific coal seam waveguide and must be measured directly in the seam being surveyed (Stolar Horizon, Inc., 2004). 
During the calibration testing, the TX is positioned stationary at one side of the physical model (headgate entry of the longwall panel) and the RX units are carried down at the other side (tailgate entry of the longwall panel), pausing at each RX station to measure the strength of the received radio signal. These intervals generally cover ray-path lengths in the model where there is no geological anomaly.

The calibration parameters are estimated by plotting signal strength as a function of ray-path length (separation of transmitter and receiver). The SAR and C-Factor are determined by evaluating the measured signal strength versus propagation distance, after correcting for cylindrical/spherical spreading and antenna pattern. The slope of the best-fit line for signal versus distance is the SAR. The SAR is calculated to establish how the RIM signal strength is attenuated as it travels through portions of coal as a function of ray-path length. This rate is normalized by 100 feet to yield the conventional rate in $\mathrm{dB}$ per 100 -foot. The C-Factor approximates the signal strength at 0 feet from the TX, i.e., the TX strength at the origin of any ray path (Stolar Horizon, Inc., 2004).

Figure 3.12 is an example of the calibration result in a longwall panel (Stolar Horizon, Inc., 2004). The panel calibration testing produced a SAR of $5.7 \mathrm{~dB} / 100 \mathrm{ft}$. The calibration data yielded a C-Factor of $150 \mathrm{~dB}$. During the calibration testing, $102.5 \mathrm{kHz}$ was the highest frequency capable of penetrating the panel, while maintaining high signal levels.

In the laboratory physical model testing, a homogeneous simulated coal seam will be made in the physical model, and calibration testing also should be done before the RIM test and the Standard Attenuation Rate (SAR) and Transmitter Coupling Factor (C-Factor) also should be determined. These parameters are unique to the specific laboratory physical model and will be used in the RIM testing data analysis. 


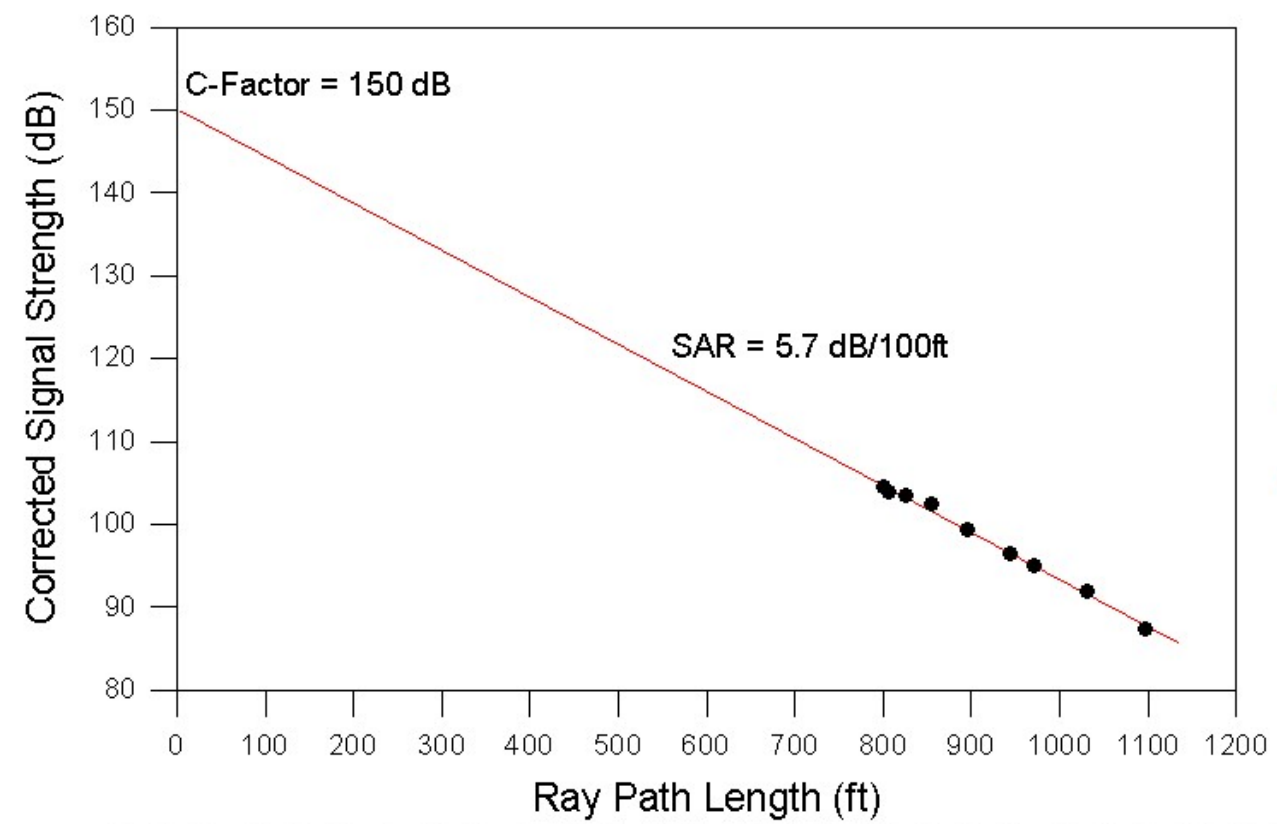

Figure 3.12 102.5-kHz calibration testing results at a longwall panel (Stolar Horizon, Inc., 2004)

\subsubsection{Reconnaissance Testing Method}

Reconnaissance testing is an initial method which can be used to quickly and primarily detect the geological anomalies in the coal seam. A reconnaissance testing layout is shown in Figure 3.13. The transmitter antenna and receiving antenna were put on separate sides of the model during testing. After the data has been recorded, the antennas were moved to the next point for testing. Since the reconnaissance just needs to test a few direct ray paths, it is easy to conduct and quick to detect the preliminary location of the geological anomalies.

Figure 3.14 is an example of reconnaissance testing results from a longwall panel (Stolarczyk and Fry, 1990). From this figure, the direct ray reconnaissance testing results show that the measured signal level drops by approximately $30 \mathrm{~dB}$ when the ray paths intersect a dike. 


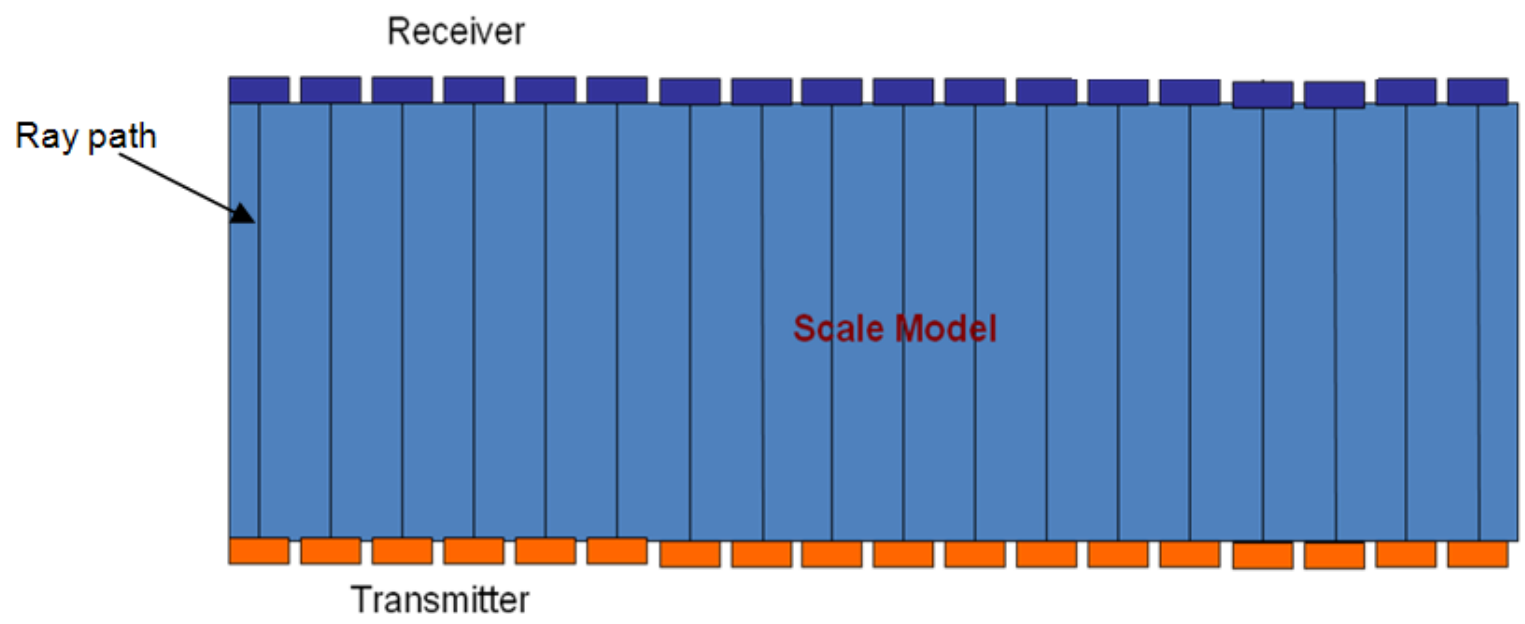

Figure 3.13 Layout of reconnaissance testing

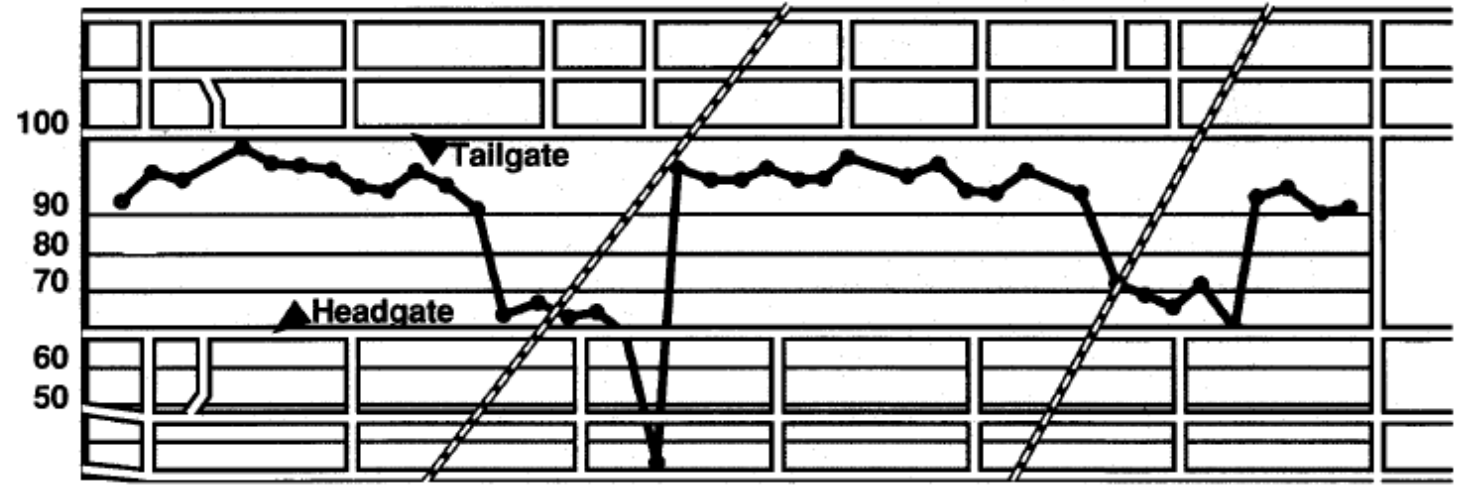

Figure 3.14 Reconnaissance testing results at a narrow panel intersected by dikes (Stolarczyk and Fry, 1990)

In the laboratory physical model test, the reconnaissance test will be done along the coal seam. The test results will be used to compare with the embedded geological anomalies. The characteristics of the reconnaissance test results (locations, signal strength/attenuation rate) should match the characteristics of the known geological anomalies. 


\subsubsection{RIM Testing Method}

The reconnaissance testing results can only roughly detect the location based on the signal strength/attenuation rate along the coal seam (cross-section view). It can not reflect the location, shapes, and signal strength/attenuation rate in the coal seam (plan view). The characteristics of the geological anomalies in plane view are more useful to understand the geological anomalies and more practical for mining engineer to design the mining plan in actual longwall mining. For example, Figure 3.15 shows reconnaissance test results along a longwall panel; the testing results indicated the location of the geological anomalies along the longwall panel. However, it is not clear what is the shape of the geological anomalies in the longwall panel and what is the attenuation rate distribution of the geological anomalies in the longwall panel. Therefore, the RIM test results and the attenuation rate tomogram (Figure 3.16) shows the characteristics of the geological anomalies which are not shown in reconnaissance. Based on the RIM testing attenuation rate tomogram and some other accessorial geology method (e.g. drilling), the mining plan of this longwall panel can be designed. So it is very important that RIM testing is conducted and the RIM attenuation rate tomogram obtained to detect the geological anomalies.

In the laboratory physical model testing, the RIM testing layout is shown in Figure 3.17 , the transmitter antenna is fixed at one position, and the receiver antenna is put on the other side of the model along the coal seam. According to radar calibration (Monaghan, 2007), the receiver antenna will receive signals that shift $90^{\circ}$ from the transmitter antenna. The number of ray paths will depend on the size of the physical model and the geological anomalies. 


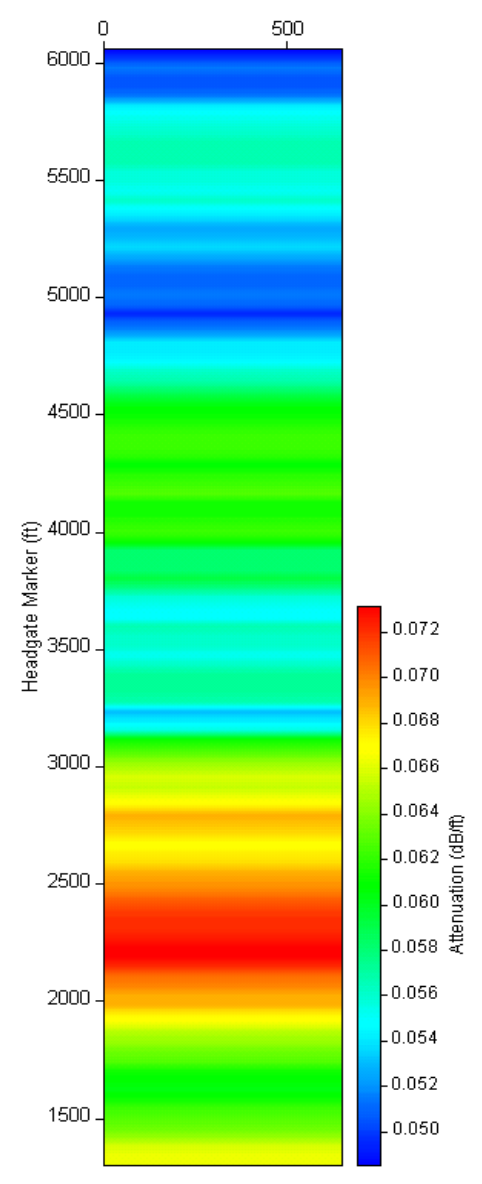

Figure 3.15 Reconnaissance testing results (Stolarczyk et al., 2006)

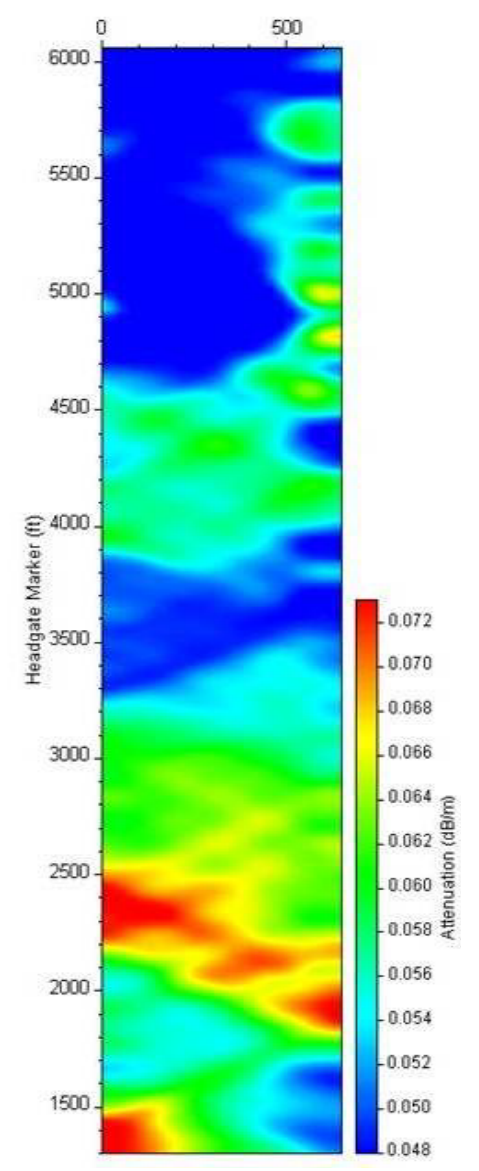

Figure 3.16 RIM testing results (Stolarczyk et al., 2006)

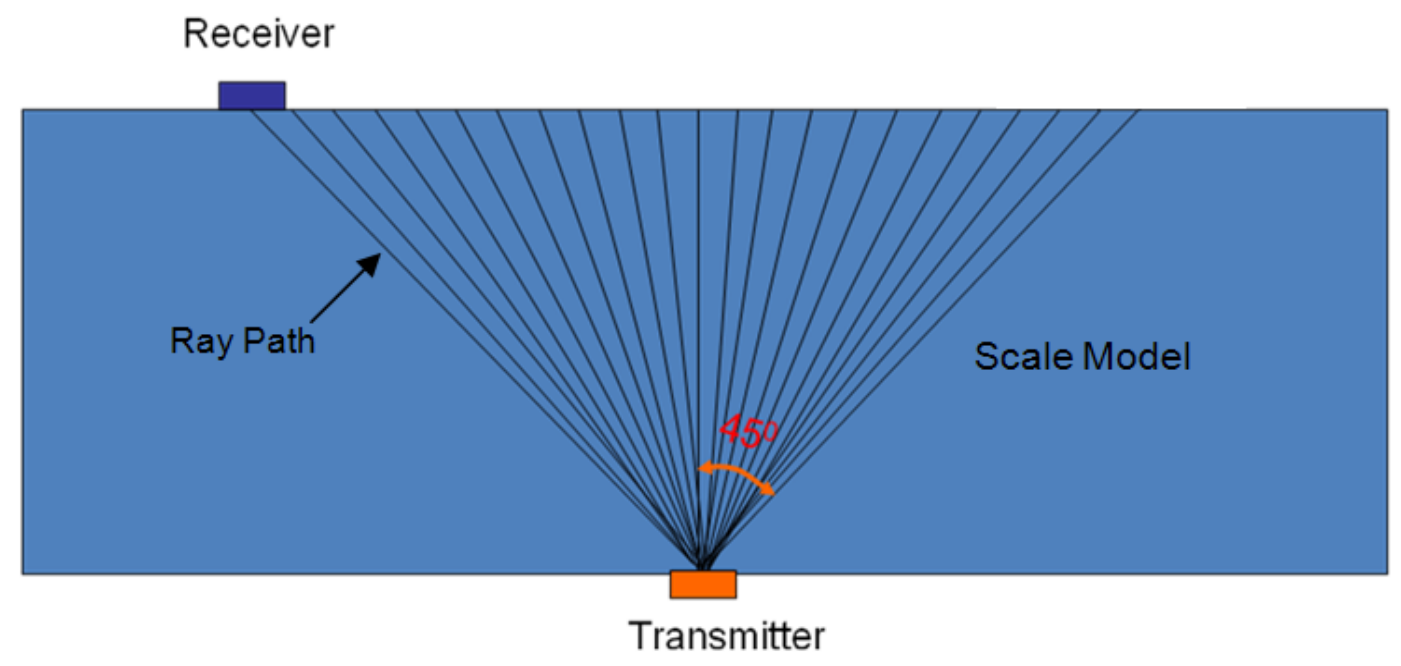

Figure 3.17 Layout of RIM testing 


\subsection{Data Format and Processing}

The raw data generated in the SIR II instrumentation is in a proprietary format file (*.DZT). In order to further post process the raw data, the DZT files were converted to ASCII (American Standard Code for Information) using a Radan to ASCII conversion utility program RTOAW.EXE (Figure 3.18). The ASCII data was then imported into a Microsoft Excel spreadsheet to obtain the average peak-to-peak values at a specific signal trace. A typical radar signal trace converted into ASCII and then plotted in Excel is shown in Figure 3.19. The Y-axis represents the signal strength maximum and minimum in counts and the $\mathrm{X}$-axis represent samples. The average amplitude value of the maximum peak and the minimum peak is acquired. This value represents the signal strength (in counts) at one testing ray path.

In order to make the data more understandable, the signal strength (in counts) is converted into volts or $\mathrm{dB}$. The values were converted to voltage as the unit based on the relationship between the amplitude (in counts) and voltage (Monaghan, 2007). Those values with voltage unit will be substituted into Eq. (2-6) used to generate and obtain the column vector $Y_{i}$ in the matrix which will be the input data in the RIM software to generate the tomogram. The detailed application of the software will be discussed in next section. The signal strength in volts can also be converted to $\mathrm{dB}$ by the following relationship:

$$
d B=20 \log \left(\frac{V_{2}}{V_{1}}\right)
$$

where $V_{1}$ and $V_{2}$ are the reference and actual voltage, respectively. 


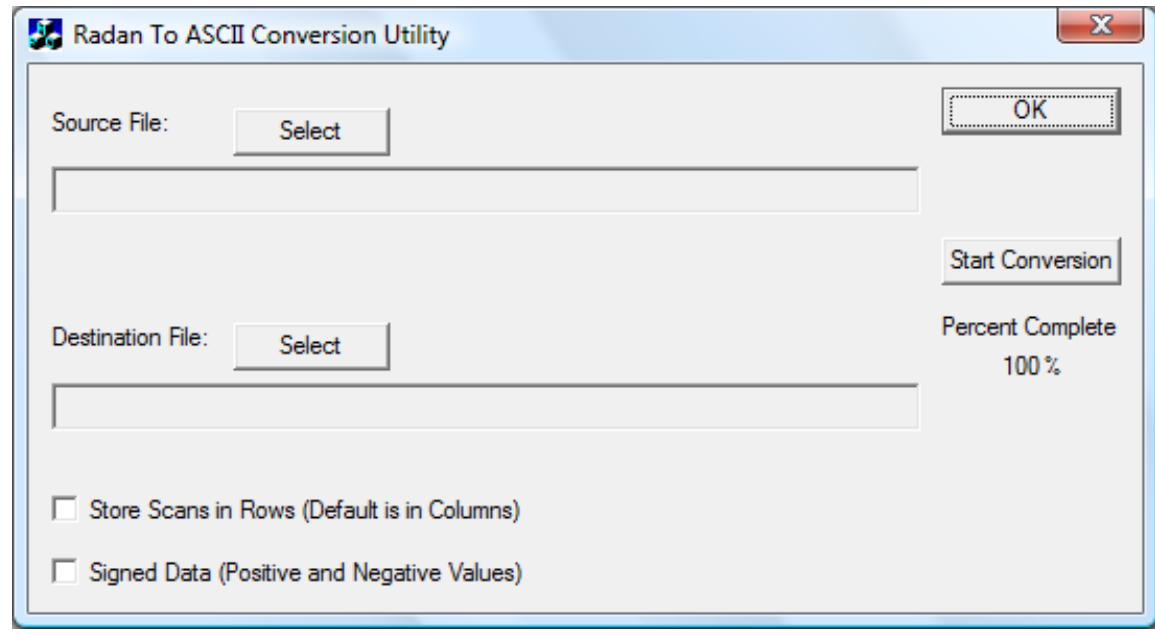

Figure 3.18 Randan to ASCII conversion utility software

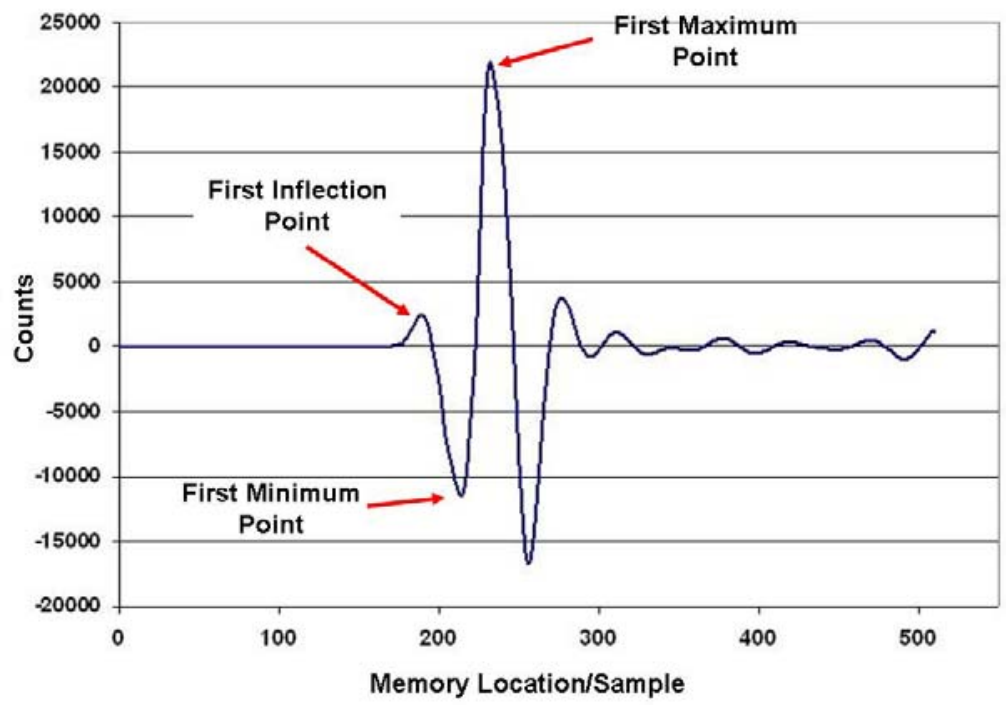

Figure 3.19 Typical converted EM waveform (Monaghan, 2007)

\subsection{RIM Tomogram Software Development and Validation}

\subsubsection{Solving for Attenuation Rates of the Laboratory Physical Model}

There are many possible methods for interpreting the transmission data. Since the coal seam supports only a single mode, the attenuation rate of the quasi-TEM (Transverse Electromagnetic) mode is the most logical quantity with which to work (Hill, 1984). 
Assuming that the coal seam parameters vary slowly, reflection and reflection are approximately neglected. The straight ray propagation in the seam can be approximately written as (Shop, 1987):

$$
H_{i}=\frac{H_{0}}{\sqrt{r_{i}}} e^{-\alpha r_{i}} \quad \text { for cylinder spreading }
$$

or

$$
H_{i}=\frac{H_{0}}{r_{i}} e^{-\alpha r_{i}} \quad \text { for spherical spreading }
$$

where $H_{0}$ is the source excitation factor depending on the transmitting antenna and the coal seam parameters, $\alpha$ is the attenuation rate in Nepers $/ m$ and depends on $x$ and $y, r_{i}$ is the length of the $i^{\text {th }}$ ray path.

The magnitude of the received signal strength $V_{i}$ for the $i^{\text {th }}$ ray path also can be written as:

$$
V_{i}=V_{0} \frac{e^{-\alpha r_{i}}}{\sqrt{r_{i}}} \quad \text { for cylinder spreading }
$$

or

$$
V_{i}=V_{0} \frac{e^{-\alpha r_{i}}}{r_{i}} \quad \text { for spherical spreading }
$$

where $V_{0}$ is a constant which depends on both antennas and seam parameters.

Since the unit of the attenuation rate $\alpha$ in equations (3-3) and (3-4) is Nepers $/ m$, it will be converted to $\mathrm{dB} / \mathrm{ft}$. Recall that the decibel is a relative unit and is defined for a measured value $X$ and a reference value $X_{\text {ref, }}$, 


$$
X(d B)=20 \log \left(\frac{X}{X_{r e f}}\right)
$$

Taking 20 logarithm of both sides of Eq. (3-3) and (3-4),

$$
20 \log \left(e^{-\alpha r_{i}}\right)=20 \log \left(\frac{V_{i}}{V_{0}} \sqrt{r_{i}}\right) \quad \text { for cylinder spreading }
$$

or

$$
20 \log \left(e^{-\alpha r_{i}}\right)=20 \log \left(\frac{V_{i}}{V_{0}} r_{i}\right) \quad \text { for spherical spreading }
$$

The attenuation rate that appears in the exponent of $e$ has units of Nepers $/ \mathrm{m}$. The following identity converts the attenuation rate to units of $\mathrm{dB} / \mathrm{ft}$ (Shop, 1987),

$$
20 \log \left(e^{-\alpha r_{i}}\right)=-\alpha(d B / f t) r_{i}
$$

By rearranging Equations (3-6) and (3-7), it becomes,

$$
\alpha(d B / f t) r_{i}=V_{0}(d B)-V_{i}(d B)-10 \log \left(r_{i}\right) \quad \text { for cylinder spreading }
$$

or

$$
\alpha(d B / f t) r_{i}=V_{0}(d B)-V_{i}(d B)-20 \log \left(r_{i}\right) \quad \text { for spherical spreading }
$$

In geophysical tomogram (Bates et al., 1942, 1981 and Durst, 1975), the usual approach is to divide the intervening region into some large number of cells over which $\alpha$ is assumed to be constant. In that case the path integral in Eq. (3-8) and (3-9) can be written as

$$
\sum_{j}^{N} D_{i j} X_{j}=Y_{i} \quad i=1,2,3, \ldots, M
$$

where $Y_{i}=V_{0}-V_{i}-10 \log \left(r_{i}\right)$ or $Y_{i}=V_{0}-V_{i}-20 \log \left(r_{i}\right)$ depends on which kind of signal spreading is used, $X_{j}$ equals the unknown attenuation rate $\alpha$ for the $j^{\text {th }}$ cell, $D_{i j}$ 
equals the length of the $i^{\text {th }}$ ray through the $j^{\text {th }}$ cell shown in Figure 2.14, and $V_{i}$ is the magnitude of the measured voltage for the $i^{\text {th }}$ ray path. Since Eq. (3-5) applies to all rays, the total system of equations can be written in the following matrix forms:

$$
\left[D_{i j}\right]\left[X_{j}\right]=\left[Y_{i}\right]
$$

where $\left[Y_{i}\right]$ is a column vector with $N^{2}$ elements corresponding to the $M$ transmission measurements in Figure 2.14. The number of elements $N^{2}$ in the unknown column matrix $\left[X_{j}\right]$ equals the number of cells, and normally $N^{2}$ is chosen equal to or somewhat less than $M$.

In a longwall panel RIM testing, the testing ray paths are huge. Therefore, there are tons of data need to be processed and analyzed to generate the RIM attenuation tomogram. The basic mathematical method of RIM tomogram method has been discussed in Section 2.3. In order to generate the RIM attenuation rate tomogram, the most important step is to solve the matrix Eq. (3-11) which needs some reconstruction algorithm to process.

Most of the tomogram software is developed based on some common reconstruction algorithms such as ART, SIRT and LSQR. Newman (1995) has developed a tomogram reconstruction algorithm called Full Wave Inversion Code (FWIC) which can be used in RIM tests. Stolarczyk and Peng (2003) compared the ART and FWIC algorithm image which shows a significant improvement in resolution. However, this software is not available for use in this research.

In order to process the RIM testing data from the laboratory physical model, RIM attenuation rate tomogram software (MiniRIM) has been developed in Matlab. The development of this software is based on the RIM tomogram method which was discussed in Section 2.3. 
Figure 3.20 shows the interface of the software. The input data include "Input Panel Parameters" (length and width of scale model), "Input Cell Parameters" (length and width defined in the RIM software), and "Load RIM Data" (column vector $Y_{i}$ in Eq. (3-6)). The most important input data is the column vector $Y_{i}$ which is calculated by $Y=V_{0}-V_{i}-20 \log \left(r_{i}\right)$. After input all the required data, two calculation methods (ART and SIRT) can be selected to calculate and generate the attenuation rate tomogram. The attenuation rate data also can be exported into EXCEL which contains the coordination and attenuation information of each individual cell for further analysis. The reconstructed tomogram can also be further adjusted through the main toolbar such as "Edit", "View", "Insert", and "Tools" etc. on the interface.

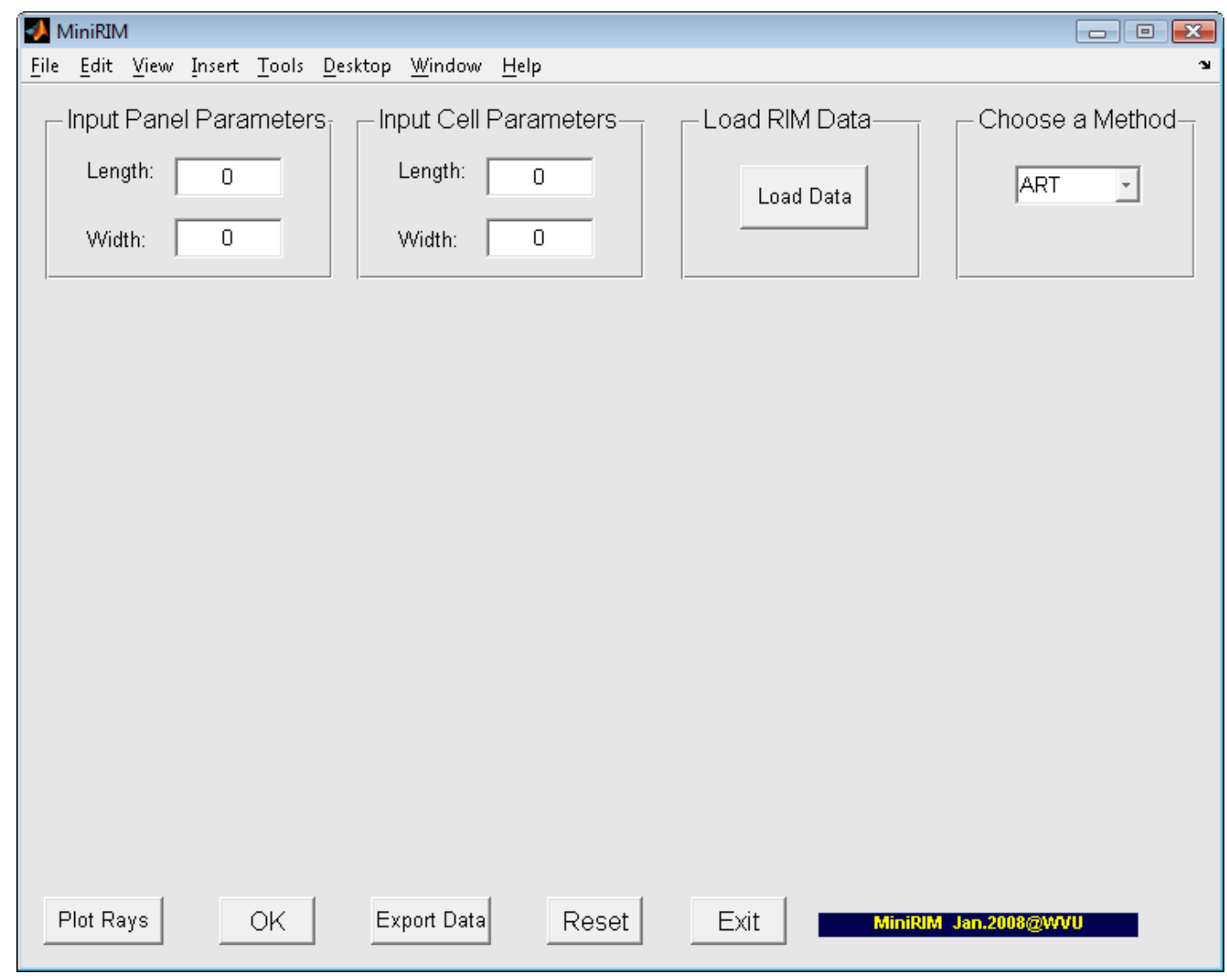

Figure 3.20 Interface of RIM tomogram software 


\subsubsection{Convergence Criteria}

The iterative reconstruction algorithm must be monitored as the iterations progress. This allows the iterations to be stopped when an acceptable solution is found. There were three such convergence criteria proposed by Gordon et al. (1970).

The first convergence criterion is Euclidean distance which is shown in Eq.(3-12).

$$
\delta=\frac{1}{N \times M} \sum_{i}\left[\Delta Y_{i}\right]^{2}
$$

where $N$ is the number of cells, $M$ is the number of ray paths, and $\Delta Y_{i}$ is the difference of estimate data vector $\left(Y^{\prime}\right)$ and the actual measured data vector $(Y)$.

The second convergence criterion is Entropy statistics, which measure the degree of disorder of attenuation rate image, or,

$$
S=\frac{1}{\ln (N \times M)} \sum_{i}\left(\alpha_{i} / \bar{\alpha}\right) \ln \left(\alpha_{i} / \bar{\alpha}\right)
$$

where $\bar{\alpha}$ is the average attenuation rate which is given by,

$$
\bar{\alpha}=\frac{1}{N \times M} \sum_{i} \alpha_{i}
$$

The third convergence criterion is Variance, which monitors the degree of variance between the ith ray path attenuation rate and the average attenuation rate, or,

$$
V=\sum_{i}\left(\alpha_{i}-\bar{\alpha}\right)^{2}
$$

From the study of Shop (1987), both the Entropy statistics criterion and the Variance criterion are not appropriate to coal seam tomography because of their limitation. The Euclidean distance criterion directly measures the agreement between the reconstructed image and the measured data. It is more appropriate for coal seam tomography. However, this criterion is not completely satisfactory because, the magnitude of this statistic is a 
function of the actual attenuation values and the size of the image. A convergence indicator, independent of the image size and data, is needed. So the new convergence criterion was developed by modifying the Euclidean distance formula to provide an average error-per-ray statistic in equation 3.16

$$
\bar{\delta}=\frac{100 \%}{N \times M}\left[\sum_{i}\left(\frac{\Delta Y_{i}}{Y_{i}}\right)^{2}\right]^{0.5}
$$

This statistic is independent of the number of rays and the magnitude of the attenuation rates and can be used to compare the convergence of two different data sets. A typical curve of the average error-per-ray versus iteration index for a SIRT algorithm is given in Figure 3. 21. This curve often has a slight divergence around the first few iterations because the initial guess data has large differences with the measured data. Then the curve rapidly converges to the best solution. Further iterations have small divergence values and little improvement for the image reconstruction.

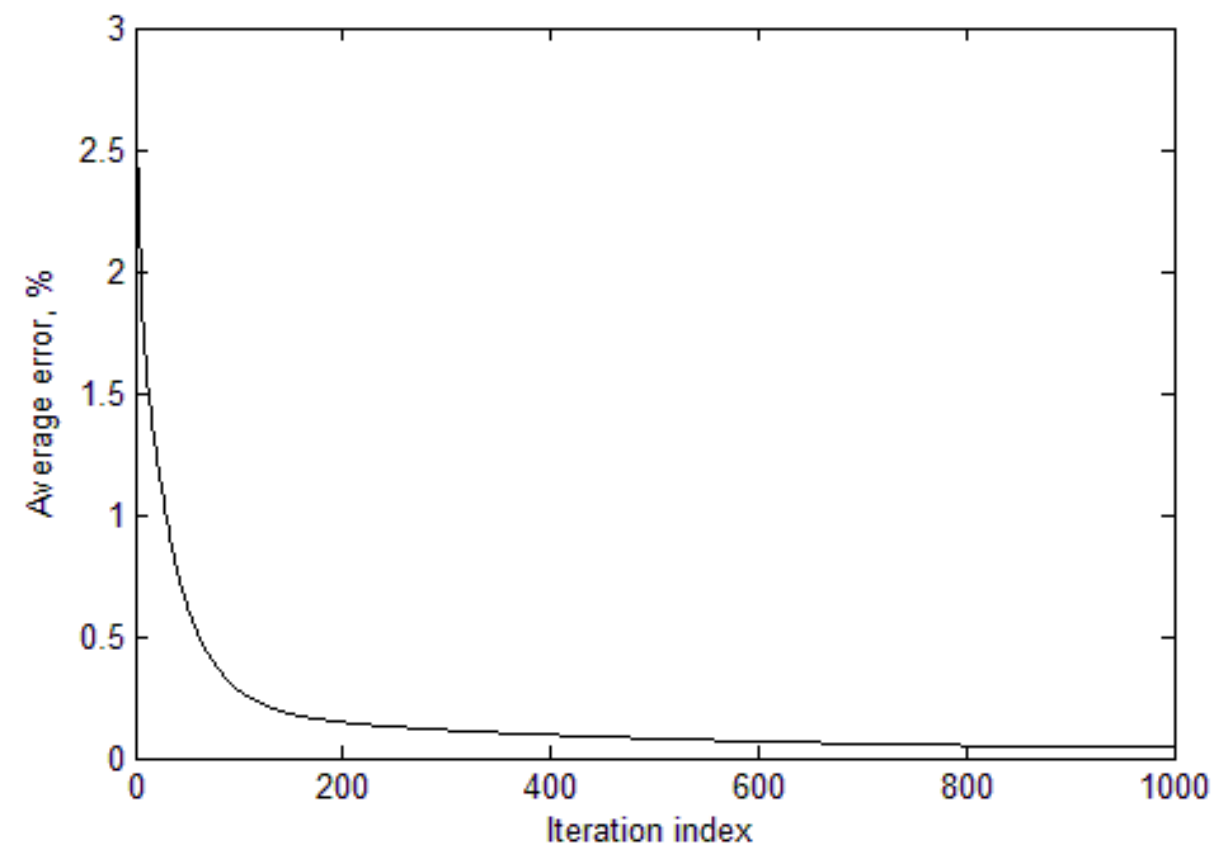

Figure 3.21 An example of a SIRT algorithm converging to an asymptotic average error-perray 


\subsubsection{Forward Modeling}

In order to validate the RIM software, five types of artificial models with different shapes of geological anomalies have been analyzed by forward modeling, and only the tomogram obtained from SIRT is discussed. In the forward modeling, the artificial coal seam and geological anomalies are generated by assigning different attenuation rates to each element. In each of the models, ten source stations (transmitter) and ten receiving stations (receiver) are spaced evenly along the opposite sides of the hypothetical panel. This results in a panel grid of 100 cells. For all the models, the attenuation rates in the anomalies are $20 \mathrm{~dB} / 100 \mathrm{ft}$ compared to a background attenuation rate of $5.0 \mathrm{~dB} / 100 \mathrm{ft}$ in the coal seam, but the shapes of the anomalies are different.

\section{Model A}

Model A is shown in Figure 3.22. The geological anomaly lies vertically at the center of the model and penetrates through the panel. The attenuation rate of the anomaly and the coal seam are $20 \mathrm{~dB} / 100 \mathrm{ft}$, and $5 \mathrm{~dB} / 100 \mathrm{ft}$, respectively. Figure 3.23 shows the tomogram from the forward modeling. It clearly shows the location and shape of the geological anomaly in the model. The data derived from the software is shown in Table 3.1, and the relative error for each cell is shown in Table 3.2. The total average relative error for the entire cell is just $1.23 \%$. It shows that the anomaly is well reconstructed after iteration with the SIRT method. 


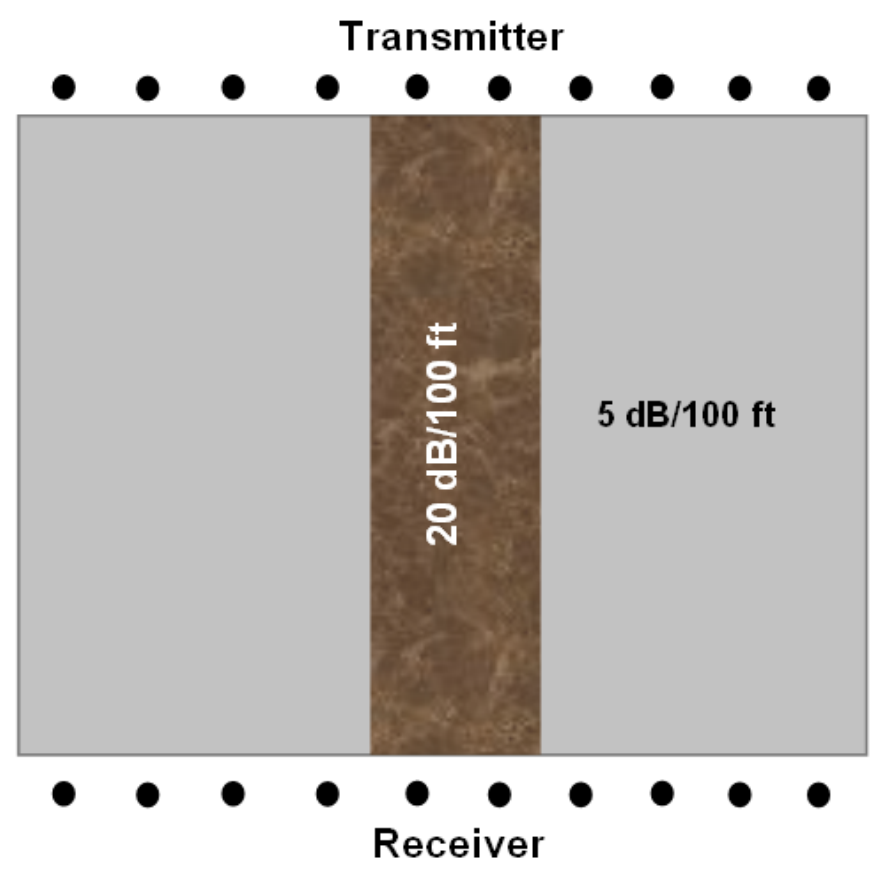

Figure 3.22 Layout of Model A

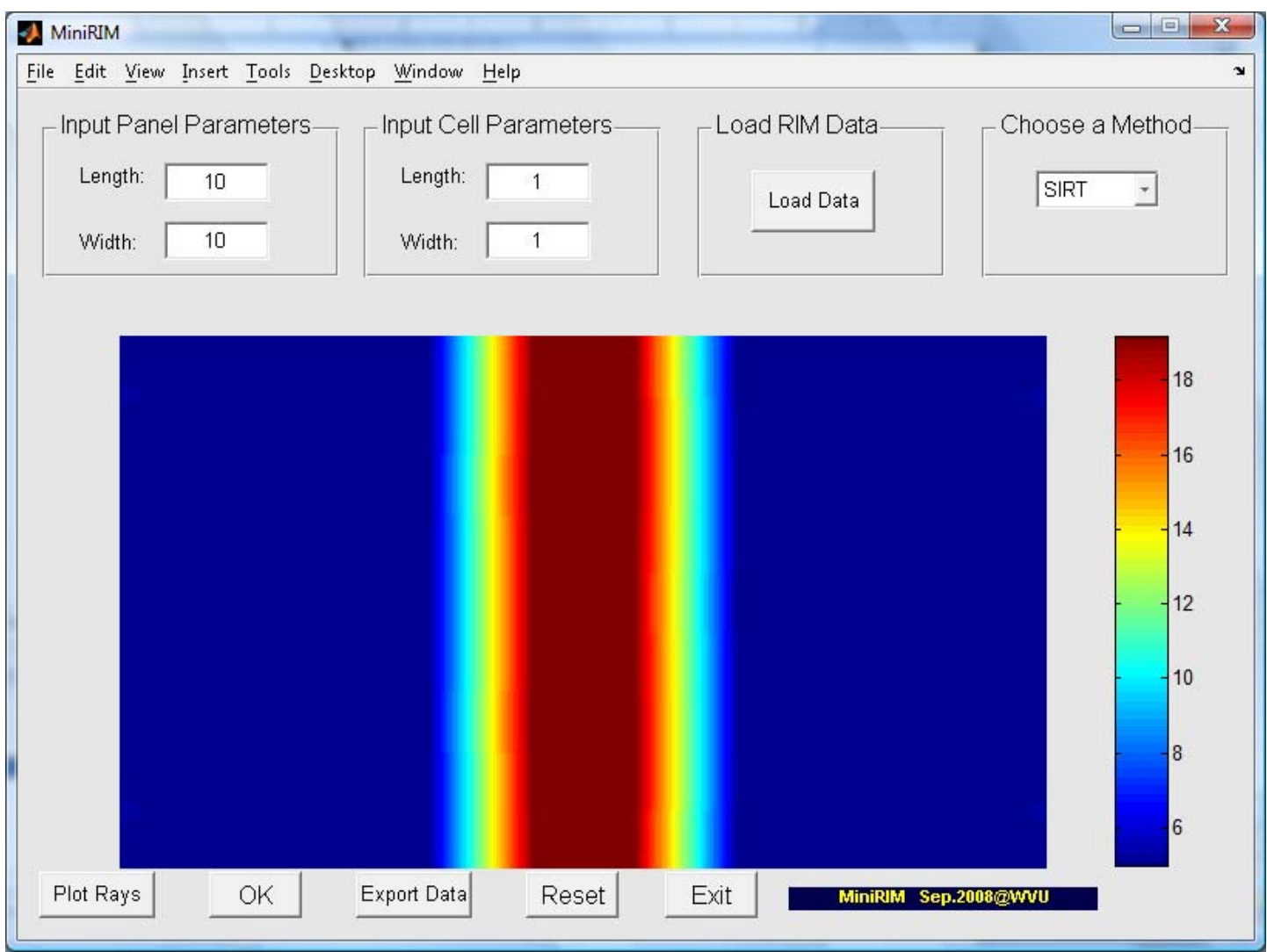

Figure 3.23 Forward modeling tomogram of Model A 


\section{Table 3.1 Forward modeling tomogram data of Model B}

\begin{tabular}{|c|c|c|c|c|c|c|c|c|}
\hline 5.0 & 5.1 & 5.0 & 5.0 & \begin{tabular}{l|l} 
& 19.9
\end{tabular} & 5.0 & 5.0 & 5.1 & 5.0 \\
\hline 5.3 & 5.0 & 4.9 & 4.9 & 20.020 .0 & 4.9 & 4.9 & 5.0 & 5.3 \\
\hline 5.0 & 4.9 & 5.0 & 5.0 & \begin{tabular}{|l|l|}
20.1 & 20.1
\end{tabular} & 5.0 & 5.0 & 4.9 & 5.0 \\
\hline 4.9 & 5.0 & 5.0 & 5.1 & \begin{tabular}{|l|l}
20.1 & 20.1
\end{tabular} & 5.1 & 5.0 & 5.0 & 4.9 \\
\hline 4.9 & 5.0 & 5.1 & 5.0 & 20.020 .0 & 5.0 & 5.1 & 5.0 & 4.9 \\
\hline 4.9 & 5.0 & 5.1 & 5.0 & $\begin{array}{ll}20.0 & 20.0\end{array}$ & 5.0 & 5.1 & 5.0 & 4.9 \\
\hline 4.9 & 5.0 & 5.0 & 5.1 & \begin{tabular}{|l|l|l}
20.1 & 20.1
\end{tabular} & 5.1 & 5.0 & 5.0 & 4.9 \\
\hline 5.0 & 4.9 & 5.0 & 5.0 & 20.120 .1 & 5.0 & 5.0 & 4.9 & 5.0 \\
\hline 5.3 & 5.0 & 4.9 & 4.9 & \begin{tabular}{|l|l|}
20.0 & 20.0
\end{tabular} & 4.9 & 4.9 & 5.0 & 5.3 \\
\hline 5.0 & 5.1 & 5.0 & 5.0 & \begin{tabular}{|l|l}
19.9 & 19.9
\end{tabular} & 5.0 & 5.0 & 5.1 & 5.0 \\
\hline
\end{tabular}

Table 3.2 Relative error of forward modeling tomogram data of Model B

\begin{tabular}{|l|l|l|l|l|l|l|l|l|l|}
\hline $0.3 \%$ & $1.9 \%$ & $0.9 \%$ & $0.8 \%$ & $0.5 \%$ & $0.5 \%$ & $0.8 \%$ & $0.9 \%$ & $1.9 \%$ & $0.3 \%$ \\
\hline $5.5 \%$ & $0.4 \%$ & $2.8 \%$ & $2.0 \%$ & $0.2 \%$ & $0.2 \%$ & $2.0 \%$ & $2.8 \%$ & $0.4 \%$ & $5.5 \%$ \\
\hline $0.3 \%$ & $2.4 \%$ & $0.8 \%$ & $0.7 \%$ & $0.6 \%$ & $0.6 \%$ & $0.7 \%$ & $0.8 \%$ & $2.4 \%$ & $0.3 \%$ \\
\hline $2.5 \%$ & $0.5 \%$ & $0.1 \%$ & $1.5 \%$ & $0.3 \%$ & $0.3 \%$ & $1.5 \%$ & $0.1 \%$ & $0.5 \%$ & $2.5 \%$ \\
\hline $2.5 \%$ & $0.9 \%$ & $2.1 \%$ & $0.0 \%$ & $0.2 \%$ & $0.2 \%$ & $0.0 \%$ & $2.1 \%$ & $0.9 \%$ & $2.5 \%$ \\
\hline $2.5 \%$ & $0.9 \%$ & $2.1 \%$ & $0.0 \%$ & $0.2 \%$ & $0.2 \%$ & $0.0 \%$ & $2.1 \%$ & $0.9 \%$ & $2.5 \%$ \\
\hline $2.5 \%$ & $0.5 \%$ & $0.1 \%$ & $1.5 \%$ & $0.3 \%$ & $0.3 \%$ & $1.5 \%$ & $0.1 \%$ & $0.5 \%$ & $2.5 \%$ \\
\hline $0.3 \%$ & $2.4 \%$ & $0.8 \%$ & $0.7 \%$ & $0.6 \%$ & $0.6 \%$ & $0.7 \%$ & $0.8 \%$ & $2.4 \%$ & $0.3 \%$ \\
\hline $5.5 \%$ & $0.4 \%$ & $2.8 \%$ & $2.0 \%$ & $0.2 \%$ & $0.2 \%$ & $2.0 \%$ & $2.8 \%$ & $0.4 \%$ & $5.5 \%$ \\
\hline $0.3 \%$ & $1.9 \%$ & $0.9 \%$ & $0.8 \%$ & $0.5 \%$ & $0.5 \%$ & $0.8 \%$ & $0.9 \%$ & $1.9 \%$ & $0.3 \%$ \\
\hline
\end{tabular}




\section{Model B}

Model B is shown in Figure 3.24. The geological anomaly lies transversely at the center of the model with an attenuation rate of $20 \mathrm{~dB} / 100 \mathrm{ft}$, and the attenuation of the coal seam is 5 $\mathrm{dB} / 100 \mathrm{ft}$. Figure 3.25 shows the tomogram of the forward modeling result. The reconstruction tomogram is poor and the anomaly is barely discernable in the reconstruction tomogram. The data derived from the software (Table. 3.3) and the relative error (Table 3.4) also show that the anomaly is not well reconstructed after iteration with SIRT method. The relative error is very high and the total average relative error is up to $89.5 \%$. This result is expected, because as each of the 100 ray paths passes the anomaly from Figure 3.24, the path length through the anomaly by the most diagonal ray is not much greater than the path length through the anomaly by a direct ray. So for this kind of anomaly, it will be difficult to reconstruct the anomaly tomogram.

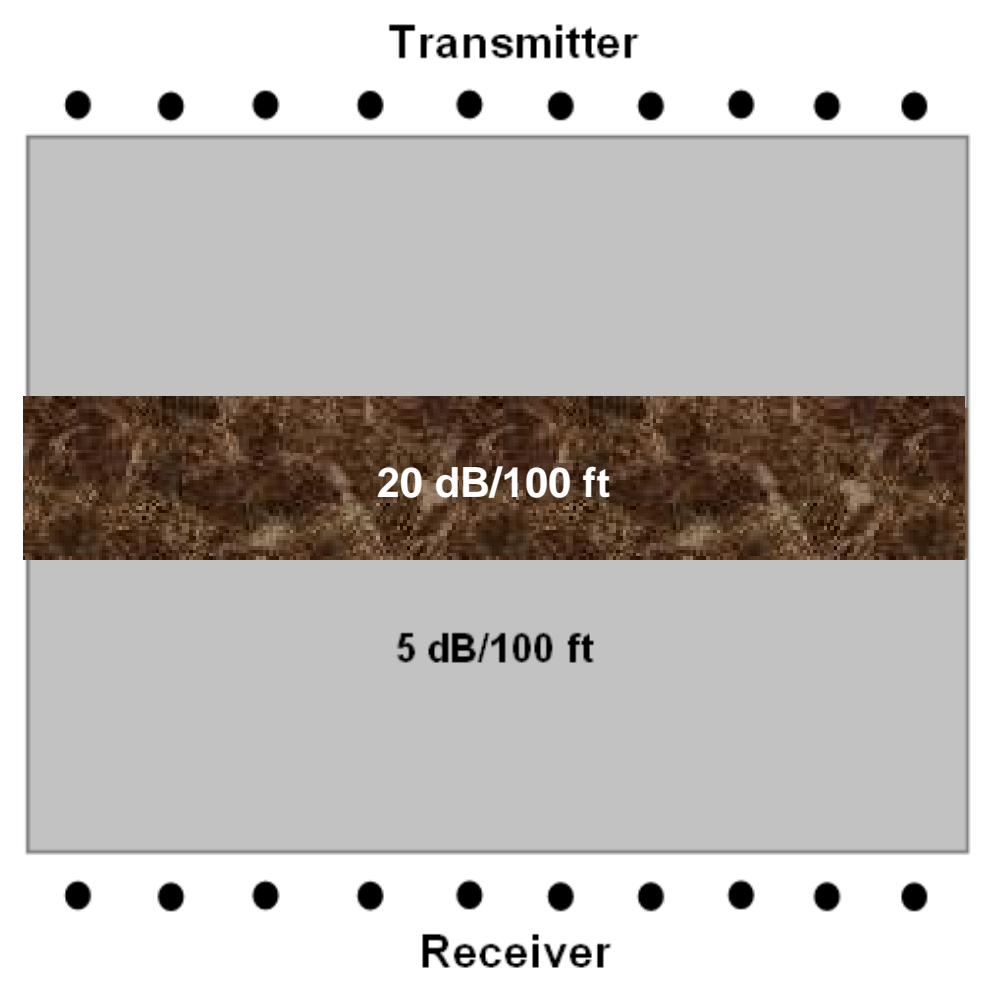

Figure 3.24 Layout of Model B 


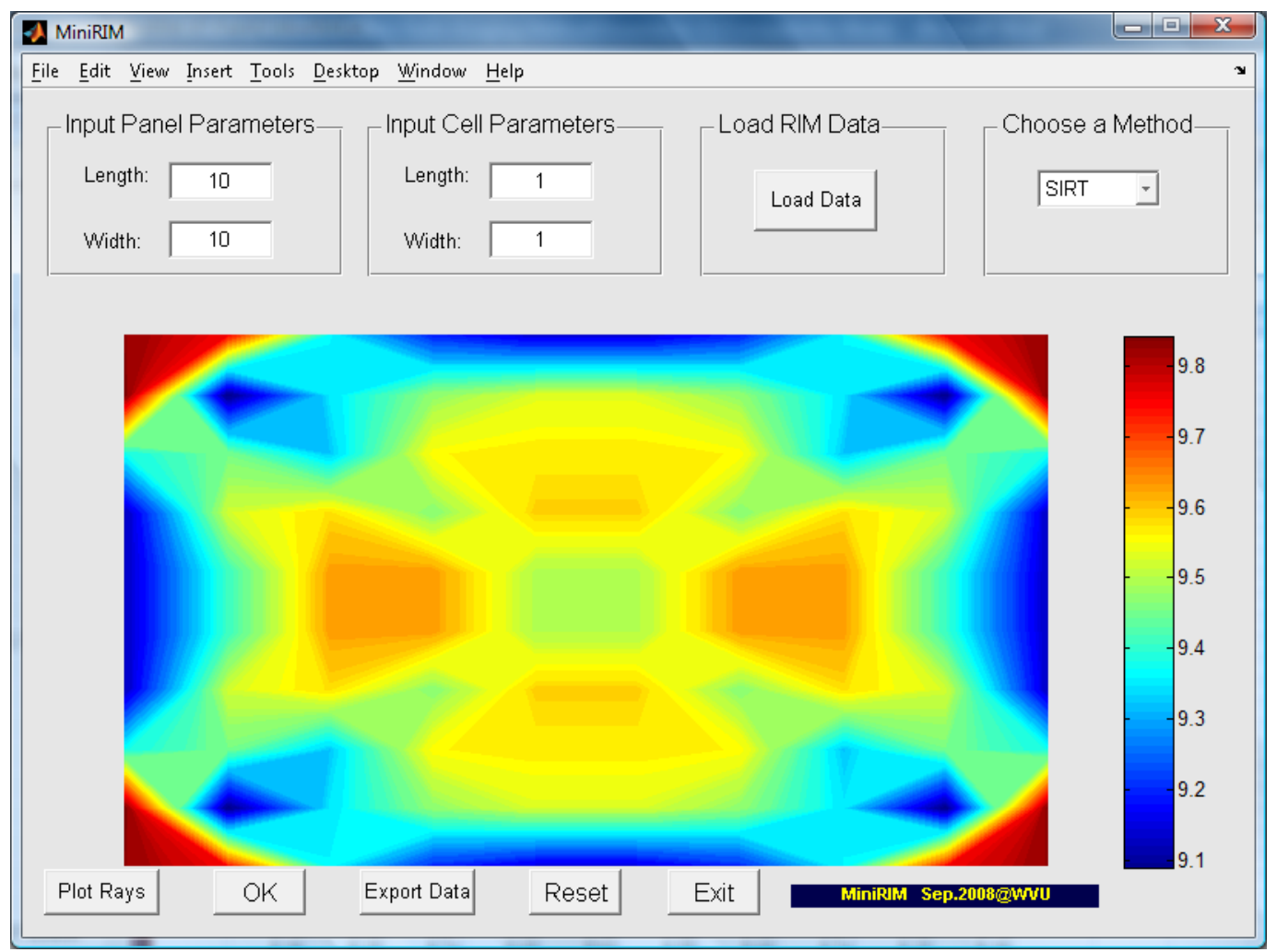

Figure 3.25 Forward modeling tomogram of Model B

Table 3.3 Forward modeling tomogram data of Model E

$\begin{array}{llllllllll}9.87 & 9.79 & 9.38 & 9.21 & 9.15 & 9.15 & 9.21 & 9.38 & 9.79 & 9.87 \\ 9.89 & 9.08 & 9.36 & 9.50 & 9.55 & 9.55 & 9.50 & 9.36 & 9.08 & 9.89 \\ 9.39 & 9.48 & 9.34 & 9.58 & 9.59 & 9.59 & 9.58 & 9.34 & 9.48 & 9.39 \\ 9.13 & 9.55 & 9.61 & 9.49 & 9.62 & 9.62 & 9.49 & 9.61 & 9.55 & 9.13 \\ 9.13 & 9.42 & 9.66 & 9.66 & 9.52 & 9.52 & 9.66 & 9.66 & 9.42 & 9.13 \\ 9.13 & 9.42 & 9.66 & 9.66 & 9.52 & 9.52 & 9.66 & 9.66 & 9.42 & 9.13 \\ 9.13 & 9.55 & 9.61 & 9.49 & 9.62 & 9.62 & 9.49 & 9.61 & 9.55 & 9.13 \\ 9.39 & 9.48 & 9.34 & 9.58 & 9.59 & 9.59 & 9.58 & 9.34 & 9.48 & 9.39 \\ 9.89 & 9.08 & 9.36 & 9.50 & 9.55 & 9.55 & 9.50 & 9.36 & 9.08 & 9.89 \\ 9.87 & 9.79 & 9.38 & 9.21 & 9.15 & 9.15 & 9.21 & 9.38 & 9.79 & 9.87\end{array}$




\section{Table 3.4 Relative error of forward modeling tomogram data of Model E}

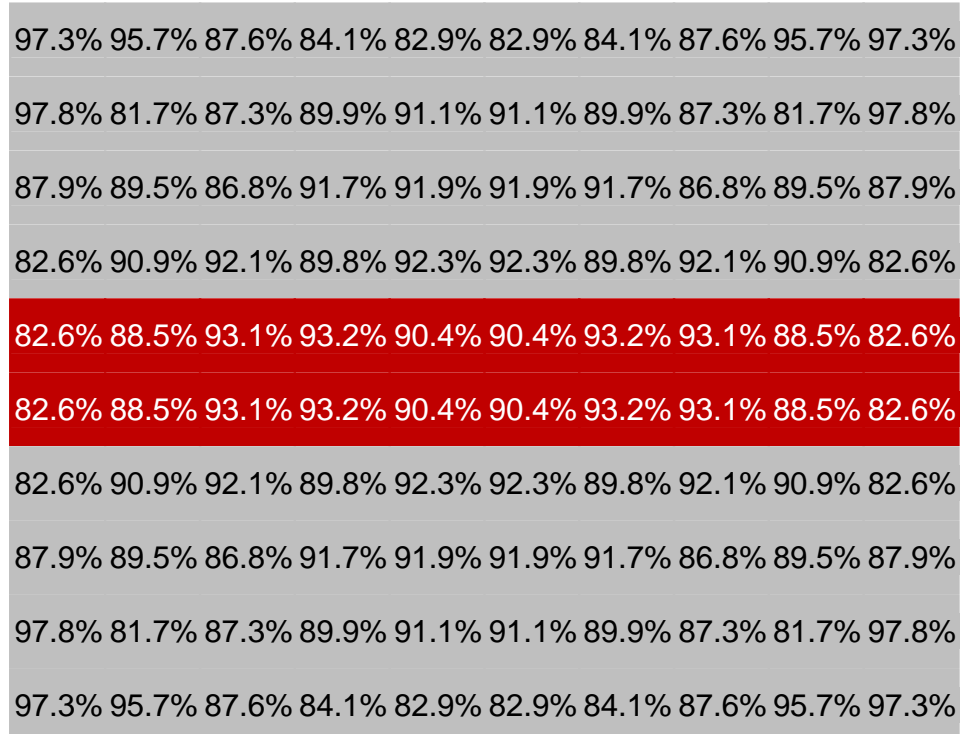

Model C

Model $\mathrm{C}$ is shown in Figure 3.26. The geological anomaly lies at the center of the model with an attenuation rate of $20 \mathrm{~dB} / 100 \mathrm{ft}$, and the attenuation of the coal seam is $5 \mathrm{~dB} / 100 \mathrm{ft}$. Figure 3.27 shows the tomogram of the forward modeling result. The tomogram also clearly shows the location and shape of the geological anomaly in the model. The data derived from the software is shown in Table 3.5, and the relative error for each cell is shown in Table 3.6. The total average relative error for the entire cell is up to $29.2 \%$, while the average relative error for the anomaly is just $4.0 \%$. It still shows that the anomaly is well reconstructed after iteration with the SIRT method. 


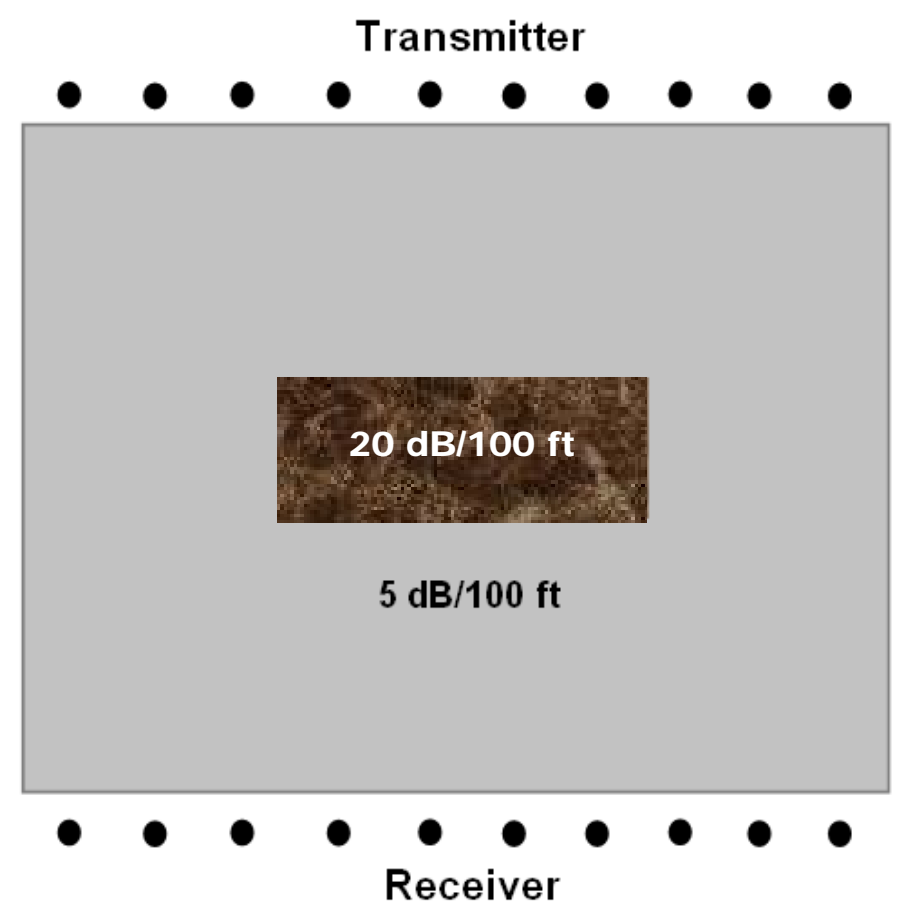

Figure 3.26 Layout of Model C

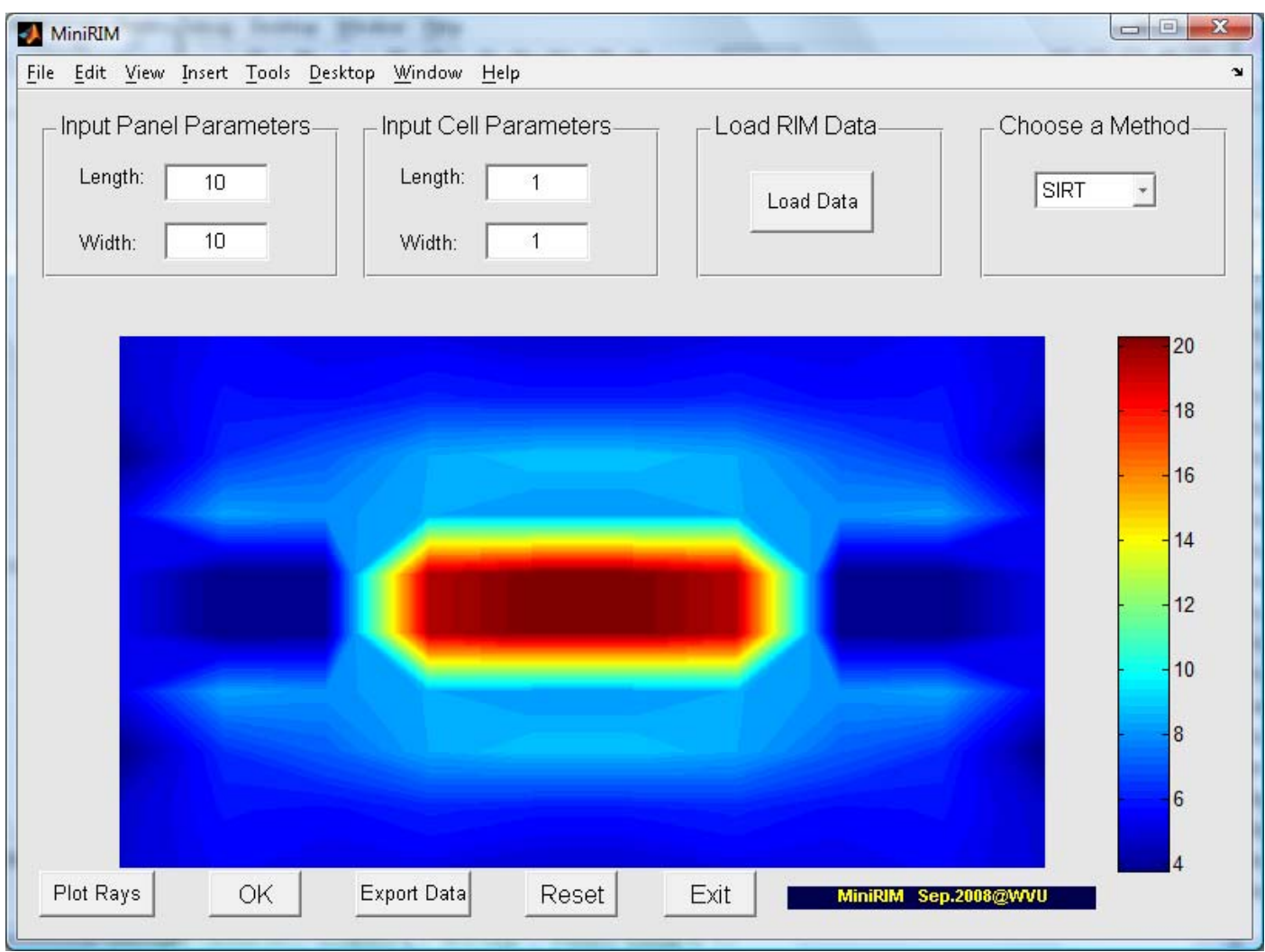

Figure 3.27 Forward modeling tomogram of Model C 
Table 3.5 Forward modeling tomogram data of Model C

$\begin{array}{llllllllll}5.0 & 5.7 & 5.4 & 5.4 & 5.1 & 5.1 & 5.4 & 5.4 & 5.7 & 5.0 \\ 4.9 & 6.0 & 5.8 & 6.3 & 6.0 & 6.0 & 6.3 & 5.8 & 6.0 & 4.9 \\ 3.7 & 6.5 & 7.4 & 8.7 & 9.1 & 9.1 & 8.7 & 7.4 & 6.5 & 3.7 \\ 5.5 & 8.6 & 8.3 & 8.7 & 8.4 & 8.4 & 8.7 & 8.3 & 8.6 & 5.5 \\ 5.5 & 3.7 & 3.9 & 20.2 & 21.3 & 21.3 & 20.2 & 3.9 & 3.7 & 5.5 \\ 5.5 & 3.7 & 3.9 & 20.2 & 21.3 & 21.3 & 20.2 & 3.9 & 3.7 & 5.5 \\ 5.5 & 8.6 & 8.3 & 8.7 & 8.4 & 8.4 & 8.7 & 8.3 & 8.6 & 5.5 \\ 3.7 & 6.5 & 7.4 & 8.7 & 9.1 & 9.1 & 8.7 & 7.4 & 6.5 & 3.7 \\ 4.9 & 6.0 & 5.8 & 6.3 & 6.0 & 6.0 & 6.3 & 5.8 & 6.0 & 4.9 \\ 5.0 & 5.7 & 5.4 & 5.4 & 5.1 & 5.1 & 5.4 & 5.4 & 5.7 & 5.0\end{array}$

Table 3.6 Relative error of forward modeling tomogram data of Model C

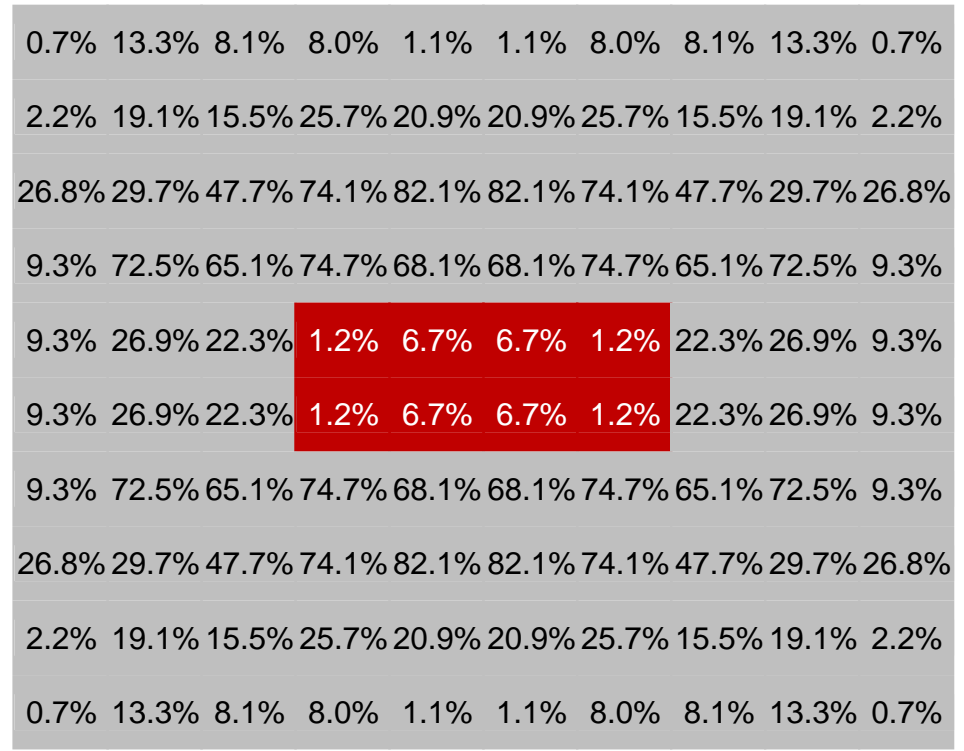




\section{Model D}

One more complicated model, D, is shown in Figure 3.28. The geological anomaly lies vertically at the center of the model with an attenuation rate of $20 \mathrm{~dB} / 100 \mathrm{ft}$, and the attenuation rate of the coal seam is $5 \mathrm{~dB} / 100 \mathrm{ft}$. Figure 3.29 shows the tomogram of the forward modeling result. The tomogram also clearly shows the location and shape of the geological anomaly in the model. The data derived from the software is shown in Table 3.7, and the relative error for each cell is shown in Table 3.8. The total average relative error for the entire cell is about $15.6 \%$. Except for the middle part of the anomaly, the relative error of other parts of the anomaly is still small. So this figure still clearly shows that the anomaly is well reconstructed after iteration with the SIRT method.

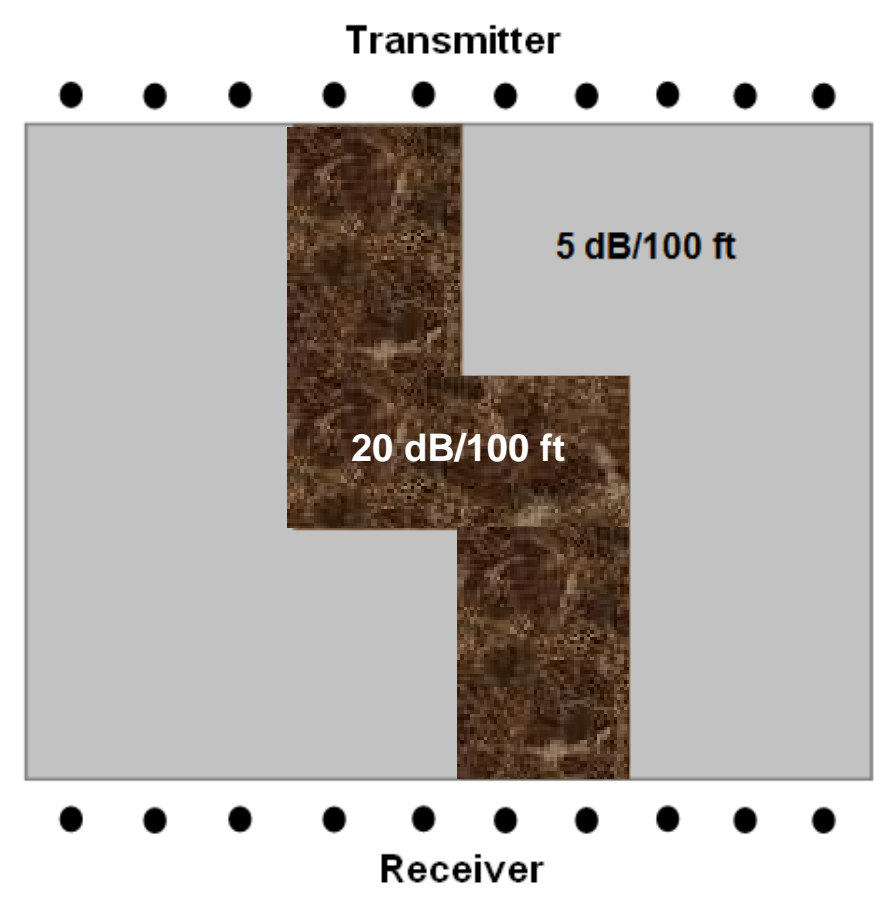

Figure 3.28 Layout of Model D 


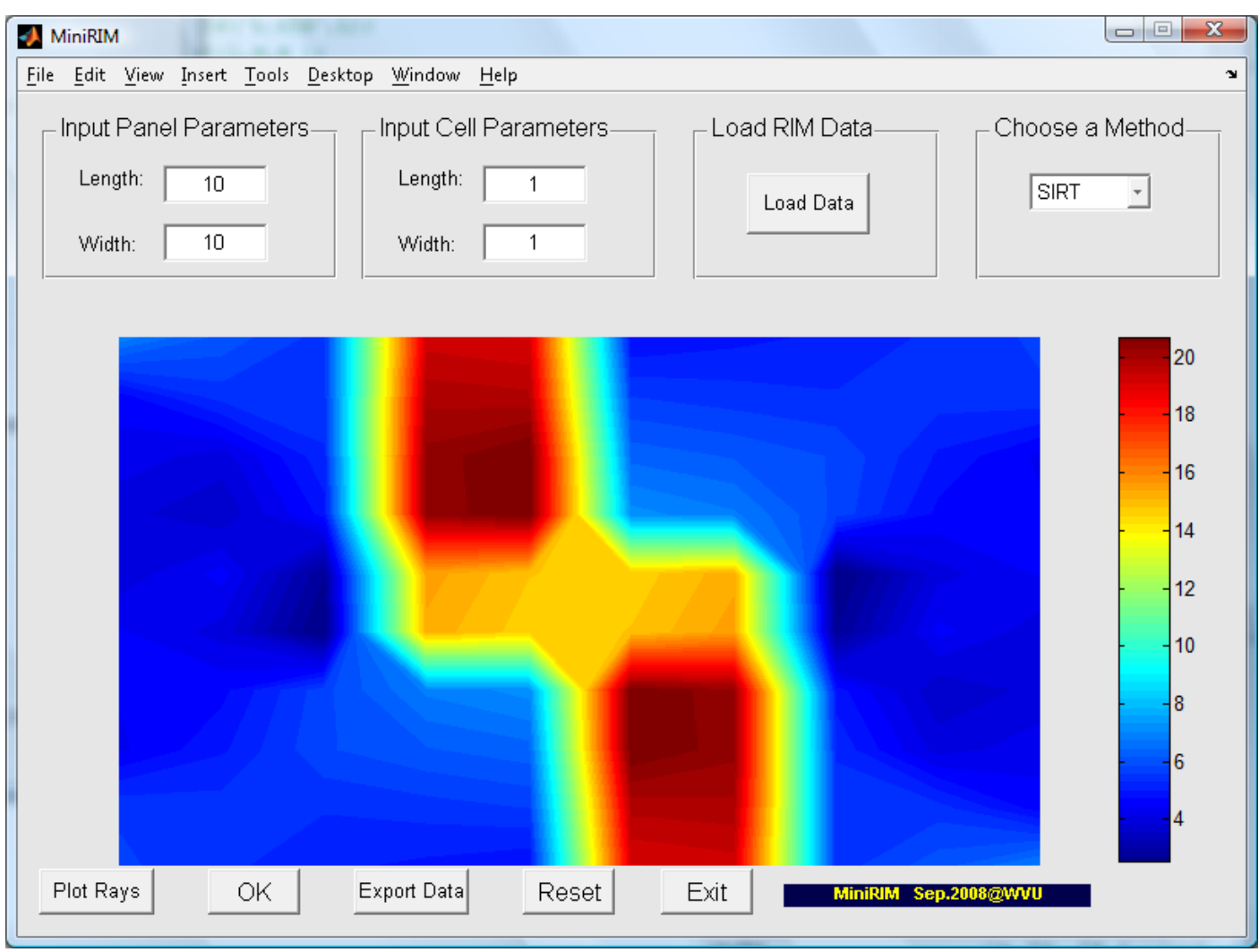

Figure 3.29 Forward modeling tomogram of Model D

Table 3.7 Forward modeling tomogram data of Model D

\begin{tabular}{|c|c|c|c|c|c|c|c|c|c|}
\hline 7.2 & 6.3 & 5.7 & 20.2 & 20.0 & 4.9 & 5.1 & 5.3 & 5.6 & 5.9 \\
\hline 4.7 & 5.6 & 5.7 & 20.8 & 20.7 & 5.8 & 5.6 & 5.5 & 5.7 & 5.8 \\
\hline 4.5 & 4.2 & 5.4 & 21.3 & 21.9 & 6.6 & 6.4 & 6.1 & 5.1 & 4.5 \\
\hline 4.1 & 3.8 & 5.0 & 21.3 & 21.9 & 7.4 & 7.1 & 6.0 & 4.8 & 4.6 \\
\hline 4.1 & 4.7 & 2.8 & 16.3 & 15.5 & 15.3 & 16.0 & 2.4 & 4.2 & 4.6 \\
\hline 4.6 & 4.2 & 2.4 & 16.0 & 15.3 & 15.5 & 16.3 & 2.8 & 4.7 & 4.1 \\
\hline 4.6 & 4.8 & 6.0 & 7.1 & 7.4 & 21.9 & 21.3 & 5.0 & 3.8 & 4.1 \\
\hline 4.5 & 5.1 & 6.1 & 6.4 & 6.6 & 21.9 & 21.3 & 5.4 & 4.2 & 4.5 \\
\hline 5.8 & 5.7 & 5.5 & 5.6 & 5.8 & 20.7 & 20.8 & 5.7 & 5.6 & 4.7 \\
\hline 5.9 & 5.6 & 5.3 & 5.1 & 4.9 & 20.0 & 20.2 & 5.7 & 6.3 & 7.2 \\
\hline
\end{tabular}


Table 3.8 Relative error of forward modeling tomogram data of Model D

\begin{tabular}{|c|c|c|c|c|c|c|c|c|c|}
\hline $43.2 \%$ & $25.8 \%$ & $13.2 \%$ & $0.8 \%$ & $0.2 \%$ & $1.9 \%$ & $2.2 \%$ & $5.4 \%$ & $11.6 \%$ & $17.3 \%$ \\
\hline $5.0 \%$ & $12.9 \%$ & $13.7 \%$ & $3.9 \%$ & $3.5 \%$ & $16.2 \%$ & $12.9 \%$ & $10.6 \%$ & $13.2 \%$ & $15.0 \%$ \\
\hline $10.8 \%$ & $16.6 \%$ & $9.0 \%$ & $6.6 \%$ & $9.3 \%$ & $31.7 \%$ & $27.4 \%$ & $21.2 \%$ & $3.0 \%$ & $9.2 \%$ \\
\hline $18.0 \%$ & $25.0 \%$ & $0.6 \%$ & $6.3 \%$ & $9.3 \%$ & $48.6 \%$ & $41.2 \%$ & $20.3 \%$ & $3.8 \%$ & $7.3 \%$ \\
\hline $18.0 \%$ & $5.7 \%$ & $43.6 \%$ & $18.7 \%$ & $22.4 \%$ & $23.4 \%$ & $20.0 \%$ & $51.6 \%$ & $16.4 \%$ & $7.3 \%$ \\
\hline $7.3 \%$ & $16.4 \%$ & $51.6 \%$ & $20.0 \%$ & $23.4 \%$ & $22.4 \%$ & $18.7 \%$ & $43.6 \%$ & $5.7 \%$ & $18.0 \%$ \\
\hline $7.3 \%$ & $3.8 \%$ & $20.3 \%$ & $41.2 \%$ & $48.6 \%$ & $9.3 \%$ & $6.3 \%$ & $0.6 \%$ & $25.0 \%$ & $18.0 \%$ \\
\hline $9.2 \%$ & $3.0 \%$ & $21.2 \%$ & $27.4 \%$ & $31.7 \%$ & $9.3 \%$ & $6.6 \%$ & $9.0 \%$ & $16.6 \%$ & $10.8 \%$ \\
\hline $15.0 \%$ & $13.2 \%$ & $10.6 \%$ & $12.9 \%$ & $16.2 \%$ & $3.5 \%$ & $3.9 \%$ & $13.7 \%$ & $12.9 \%$ & $5.0 \%$ \\
\hline $17.3 \%$ & $11.6 \%$ & $5.4 \%$ & $2.2 \%$ & $1.9 \%$ & $0.2 \%$ & $0.8 \%$ & $13.2 \%$ & $25.8 \%$ & $43.2 \%$ \\
\hline
\end{tabular}

\section{Model E}

Another complicated model E is shown in Figure 3.30. There are two geological anomalies lying at the center of the model with the attenuation rate of $50 \mathrm{~dB} / 100 \mathrm{ft}$ and $20 \mathrm{~dB} / 100 \mathrm{ft}$, respectively. The attenuation rate of the coal seam is $5 \mathrm{~dB} / 100 \mathrm{ft}$. Figure 3.31 shows the reconstruction tomogram of the forward modeling result. The tomogram also clearly shows the location and shape of the geological anomalies in the model. The data derived from the software is shown in Table 3.9, and the relative error for each cell is shown in Table 3.10. The total average relative error for the entire cell is about $19.9 \%$. Both of the anomalies tomogram with $50 \mathrm{~dB} / 100 \mathrm{ft}$ and $20 \mathrm{~dB} / 100 \mathrm{ft}$, respectively, are well reconstructed. 


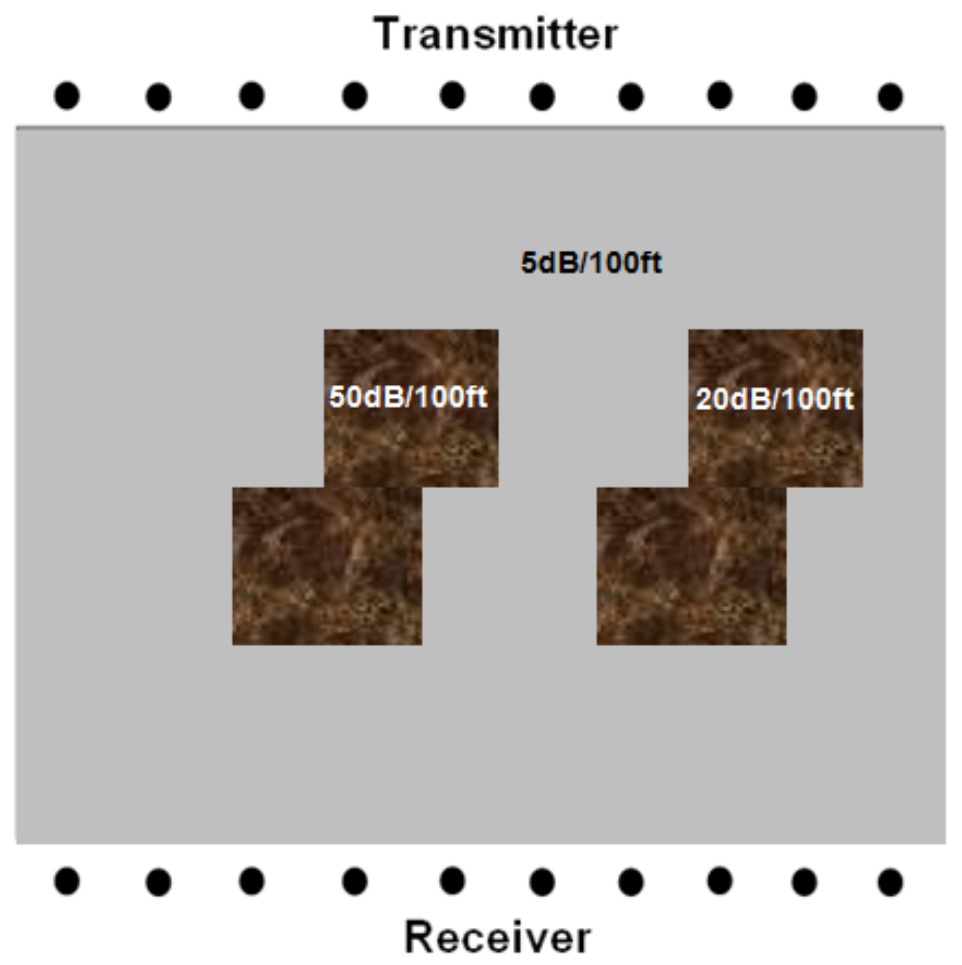

Figure 3.30 Layout of Model E

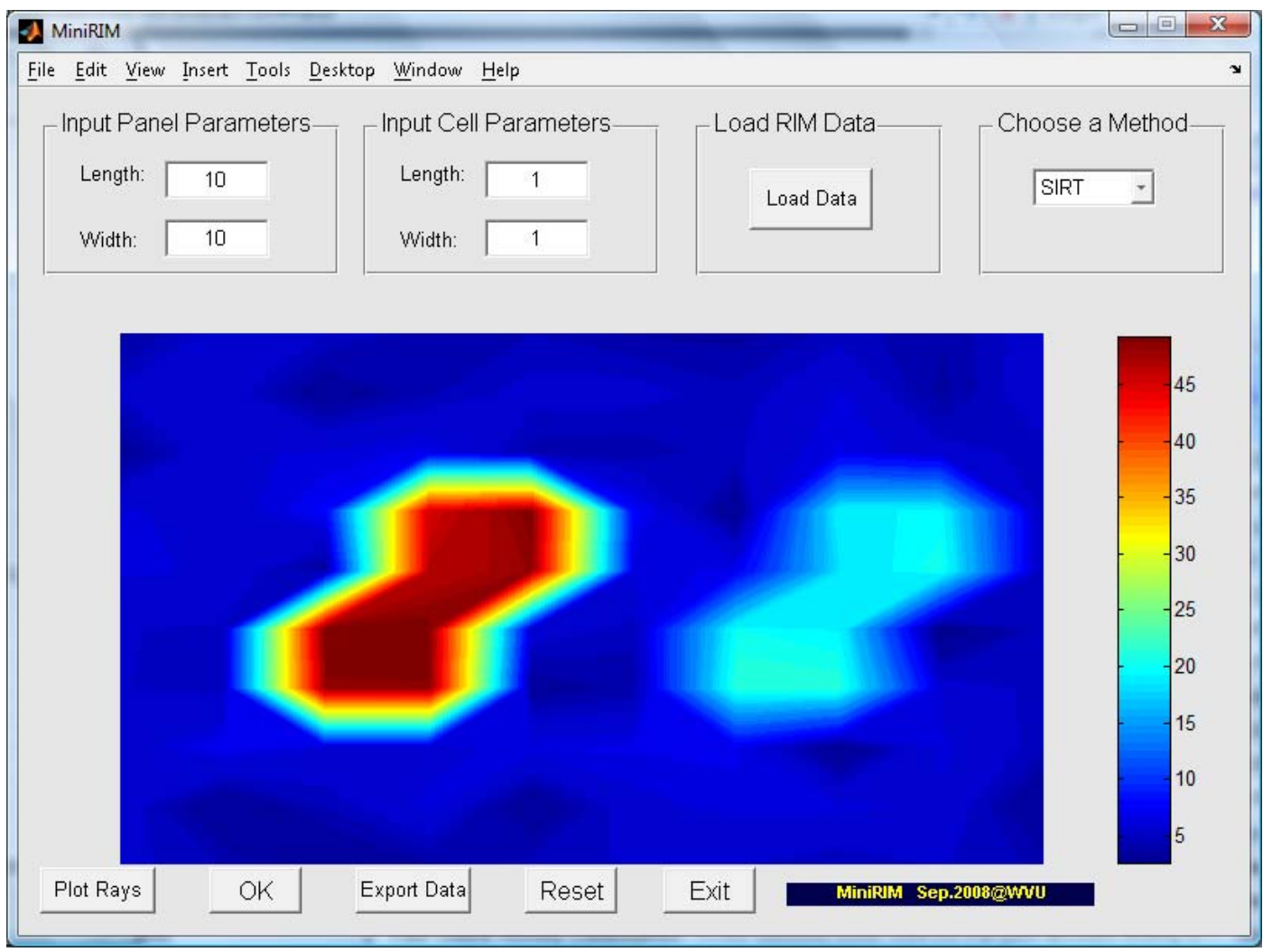

Figure 3.31 Forward modeling tomogram of Model D 


\section{Table 3.9 Forward modeling tomogram data of Model D}

\begin{tabular}{|c|c|c|c|c|c|c|c|c|c|}
\hline 4.0 & 5.9 & 5.4 & 4.4 & 4.8 & 4.2 & 4.0 & 5.2 & 4.2 & 6.1 \\
\hline 5.7 & 5.9 & 3.4 & 4.7 & 5.8 & 5.8 & 5.0 & 6.7 & 4.0 & 6.4 \\
\hline 3.5 & 6.5 & 6.7 & 7.4 & 4.5 & 4.3 & 5.3 & 7.3 & 5.9 & 5.1 \\
\hline 6.8 & 5.9 & 8.1 & 47.3 & 51.5 & 8.2 & 2.9 & 20.3 & 20.5 & 4.8 \\
\hline 6.8 & 6.4 & 3.7 & 48.8 & 50.5 & 6.8 & 7.5 & 19.1 & 21.6 & 4.8 \\
\hline 5.4 & 5.3 & 52.2 & 51.7 & 6.2 & 5.1 & 21.0 & 20.3 & 2.2 & 5.1 \\
\hline 5.4 & 5.1 & 50.7 & 50.9 & 3.5 & 4.3 & 22.3 & 22.1 & 6.1 & 5.1 \\
\hline 5.9 & 7.8 & 6.4 & 6.1 & 7.1 & 7.3 & 7.4 & 2.8 & 6.8 & 4.9 \\
\hline 6.0 & 3.0 & 4.9 & 6.9 & 3.0 & 6.2 & 6.9 & 6.8 & 6.4 & 2.8 \\
\hline 5.7 & 4.8 & 3.0 & 4.0 & 5.1 & 5.5 & 5.5 & 4.8 & 5.5 & 4.6 \\
\hline
\end{tabular}

Table 3.10 Relative error of forward modeling tomogram data of Model D

\begin{tabular}{|c|c|c|c|c|c|c|c|c|c|}
\hline $20.9 \%$ & $18.6 \%$ & $7.7 \%$ & $11.2 \%$ & $4.1 \%$ & $16.9 \%$ & $20.9 \%$ & $3.3 \%$ & $15.5 \%$ & $21.4 \%$ \\
\hline $13.4 \%$ & $18.9 \%$ & $31.4 \%$ & $6.1 \%$ & $16.5 \%$ & $16.2 \%$ & $0.5 \%$ & $34.9 \%$ & $20.2 \%$ & $28.3 \%$ \\
\hline $29.5 \%$ & $29.4 \%$ & $34.2 \%$ & $48.4 \%$ & $10.1 \%$ & $13.7 \%$ & $5.6 \%$ & $46.0 \%$ & $17.2 \%$ & $2.5 \%$ \\
\hline $36.2 \%$ & $18.3 \%$ & $61.9 \%$ & $5.4 \%$ & $3.0 \%$ & $63.9 \%$ & $42.6 \%$ & $1.6 \%$ & $2.3 \%$ & $4.5 \%$ \\
\hline $36.2 \%$ & $28.6 \%$ & $26.6 \%$ & $2.3 \%$ & $1.0 \%$ & $35.1 \%$ & $50.3 \%$ & $4.3 \%$ & $8.0 \%$ & $4.5 \%$ \\
\hline $8.6 \%$ & $6.0 \%$ & $4.3 \%$ & $3.4 \%$ & $23.6 \%$ & $2.2 \%$ & $5.2 \%$ & $1.5 \%$ & $55.9 \%$ & $2.2 \%$ \\
\hline $8.6 \%$ & $2.3 \%$ & $1.3 \%$ & $1.8 \%$ & $29.4 \%$ & $13.1 \%$ & $11.7 \%$ & $10.5 \%$ & $22.9 \%$ & $2.2 \%$ \\
\hline $18.8 \%$ & $55.2 \%$ & $28.7 \%$ & $22.2 \%$ & $42.8 \%$ & $46.4 \%$ & $48.3 \%$ & $43.7 \%$ & $35.4 \%$ & $1.1 \%$ \\
\hline $21.0 \%$ & $40.1 \%$ & $2.9 \%$ & $38.4 \%$ & $40.8 \%$ & $24.9 \%$ & $37.9 \%$ & $36.4 \%$ & $29.0 \%$ & $43.9 \%$ \\
\hline $14.2 \%$ & $4.7 \%$ & $39.3 \%$ & $19.2 \%$ & $1.8 \%$ & $9.9 \%$ & $10.4 \%$ & $3.8 \%$ & $9.7 \%$ & $7.4 \%$ \\
\hline
\end{tabular}

In conclusion, these five forward modeling results indicate that most of the anomalies can be adequately reconstructed. The geological anomaly lying vertically at the center of 
the model can produce the best reconstruction tomogram. However, for the geological anomaly lying transversely at the center of the model (Mode B), because panel anomalies cause only small attenuation differences between rays, the tomogram is poorly reconstructed. In reality, such transverse anomalies are rare in longwall panels because the panels are laid-out to perpendicularly encounter major geological trends in a mine, when possible. 


\section{CHAPTER 4 PHYSICAL MODELS TESTING AND DATA ANALYSIS}

Since the electromagnetic properties of the materials, which are used to build the physical models, have been tested, and the attenuation rate tomography software has been developed, the next step is to design and construct the physical model.

In order to eliminate the EM wave disturbance, a wood box has been constructed (Figure 4.1) to build the physical scale model. The testing and data analysis of two physical models have been constructed and analyzed.

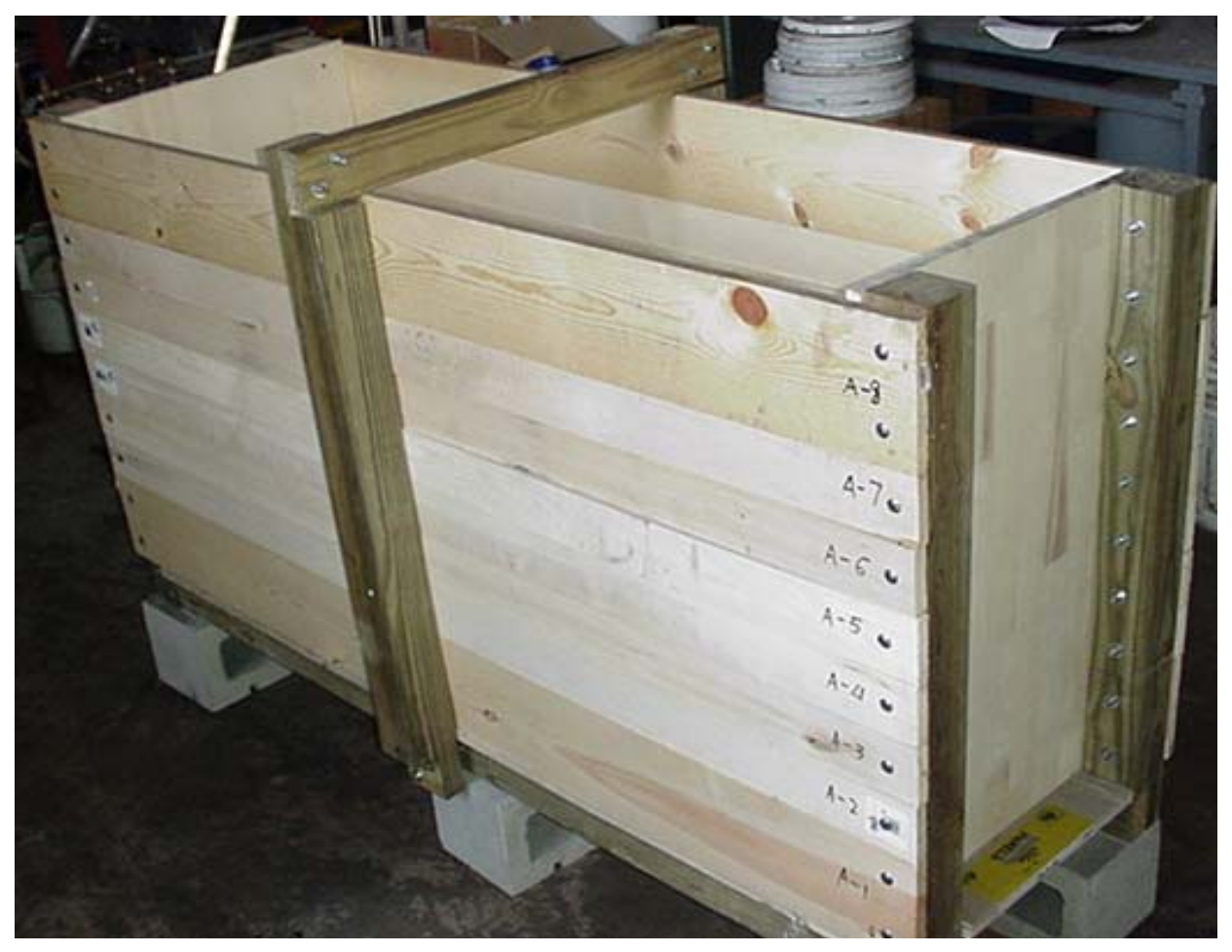

Figure 4.1 Wood box used to build the scale physical model

\subsection{First Scale Model Study}

The first model was constructed without considering the details of the EM properties of the materials of the physical model, and reconnaissance testing and RIM testing were 
performed. This preliminary study proved that it is important to select properly scaled materials to simulate the roof/floor, coal seam, and geological anomalies.

The layout of the model is shown in Figure 4.2. The roof/floor was built with a mixture of sand:gypsum:cement=8:1:1, no salt was added. The coal seam was built with a mixture of coal powder:gypsum=8:2. 10\% water was added in the mixture materials when the model was built. The reconnaissance and RIM testings were conducted 20 days after the model was built.
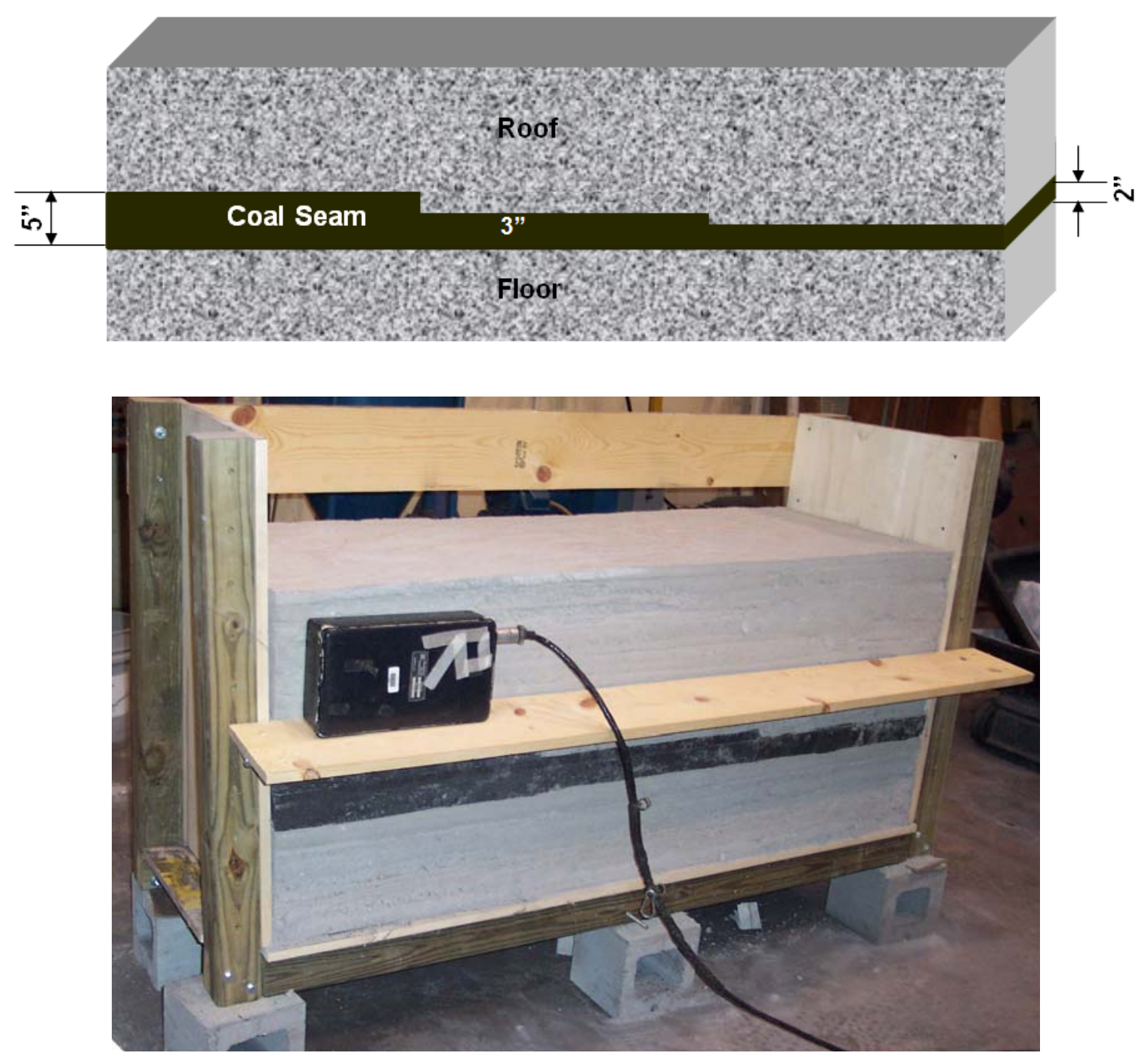

Figure 4.2 Layout of the first scale model 
Figure 4.3 shows the reconnaissance testing results for the coal seam and the floor. The signal strength has a sharp drop where the coal seam thickness changes. The average signal strength in the floor is smaller than that in the coal seam. This is what we expected because the attenuation rate in the roof/floor is bigger than that in the coal seam. However, the results from the RIM testing were not good, and it was difficult to reconstruct the tomogram that represents the location and shape of the seam thickness changes.

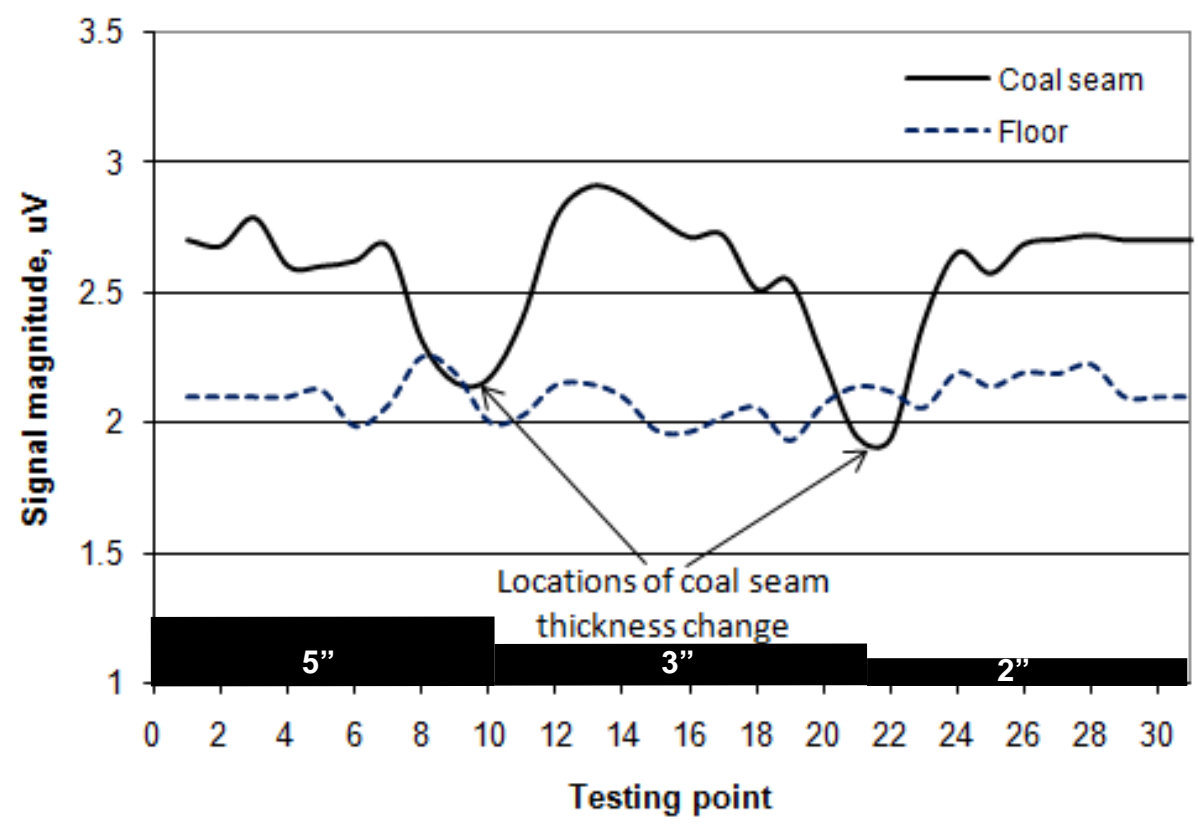

Figure 4.3 Reconnaissance test results for the coal seam and the floor

From the testing results of the first model, some factors that critically affect the test results had been analyzed. The first factor was that no salt or other materials were added in the roof/floor to increase the electrical conductivity. The difference in electrical conductivity between the roof/floor and the coal seam was insufficient. Consequently, more energy leaked into the roof/floor, and the attenuation in the thick coal seam (5 in.) and the thin coal seam (2 in.) has not much difference. The second factor was that the moisture content control. The model was tested 20 days after the model was constructed. The model 
dried slowly. The sample tests (Figure 3.3-3.8) showed that when the water content was very small, the difference in electrical conductivity and dielectric constant was very small even with different mixture ratios. In other words, the EM properties between the roof/floor and the coal seam did not differ much. Therefore it was difficult to detect changes in signal strength in the different coal seam thickness. The third factor was that the coal seam thickness in the scale model might be too small. The dimension of the $900 \mathrm{MHz}$ antennas used in the testing was about 12 in. $\times 3$ in. $\times 7$ in., so some of the energy might have leaked into the roof/floor, and the signal strength of the waveguide in the coal seam was weak.

Although the first scale model was not successful, much useful information had been obtained for further study. The EM properties of the roof/floor and the coal seam, the water content in the scale model, and the dimension of the scale model are very important factors that will affect the test results. Those parameters must be carefully considered and controlled in the next model design and construction.

\subsection{Second Scale Model Study}

\subsubsection{Scale Factors Determination}

It was learned from the first scale model study that it is very important to control the EM properties (DC and EC), water content, and scale model dimensions when building the scale models. It is necessary to test the EM properties of the materials used to construct the scale model and the water content before the scale model is constructed.

From the scale model theory and other laboratory EM scale models (Smith and Scott, 1989, King and Smith, 1981, Hyun, et al., 2007), most of the scale models selected 1:3 to 1:6 as the dimension scale factor. The scale factors in those scaled models were very close to the full-sized system, because the frequency in the full-sized system was very high (e.g. 
$500 \mathrm{MHz}$ ). It is easy to select even higher frequency radars (e.g. $1.5 \mathrm{GHz}$ ) to scale the model in the laboratory. Both the dimension of the scaled model and the very high frequency radar (smaller size) are easy to handle in the laboratory.

However, in this study, since the frequency used in the full-sized system is very low (as low as $90-290 \mathrm{KHz}$ ), the high frequency radar which is convenient for use in the laboratory is $900 \mathrm{MHz}$ or even higher. So the frequency scale factor will be up to 1:10000 (90:900000) which makes it impossible to meet the other scale factors (e.g., dimension scale factor, electrical conductivity scale factor etc.). King (1982) pointed out that the frequency and size need not be scaled inversely by the same factor. The main effects of the frequency are to change the attenuation rate and the penetration depth of the EM signals. Besides, according to Eq. 3.2, the attenuation rate obtained in the high frequency testing can be converted to that in low frequency testing. So obtain test results with high frequency but not to scale with the size scale factor will not affect the study results.

Actually, the most important factors which affect the EM wave propagation in the coal seam are the dielectric constant and electrical conductivity (see Section 3.2); these two scale factors must be satisfied in the scaled model.

In order to build a convenient scale model for tests with small size high frequency radars and at the same time meet the dielectric constant and electrical conductivity scale factors, a scale factor of 1:5 was selected to simulate parts of the longwall panel in this study. Geophysical Survey System, Inc. (GSSI) Subsurface Interface Radar (SIR)® II control unit with two $900 \mathrm{MHz}$ antennas was selected to test the scaled model.

Based on Stolar Company's testing experience, 3 wave lengths are best for the RIM tests. Considering the high frequency $(900 \mathrm{MHz})$ and the dielectric constant of coal at 3.2 
used in the laboratory scale test, the width of the coal seam in the physical model is determined as:

3 wave length $3 \lambda=3 \times \frac{v}{f}=3 \times \frac{C}{f \sqrt{\varepsilon}}=3 \times \frac{3 \times 10^{8}}{9 \times 10^{8} \times \sqrt{3.2}}=22$ in.

There is no specific requirement for the length of the physical model. 64 in. was selected for the length of the physical model.

A $3.3 \mathrm{ft}$ height of coal seam was selected in the full-sized system. The height of the coal seam in the physical model should be:

\section{$3.3 \times 12 / 5=8$ in.}

The same material was selected to simulate the roof and floor, which have a same thickness of 8 in., in the physical model.

So the total dimensions of the physical model were 64 in. $\times 22$ in. $\times 24$ in. (Figure 4.4 ). These dimensions cannot simulate the whole longwall panel, but just parts of it. Since the testing results will be represented as a tomogram of attenuation rate, even the physical model is just a part of the longwall panel, it will not affect the study results.

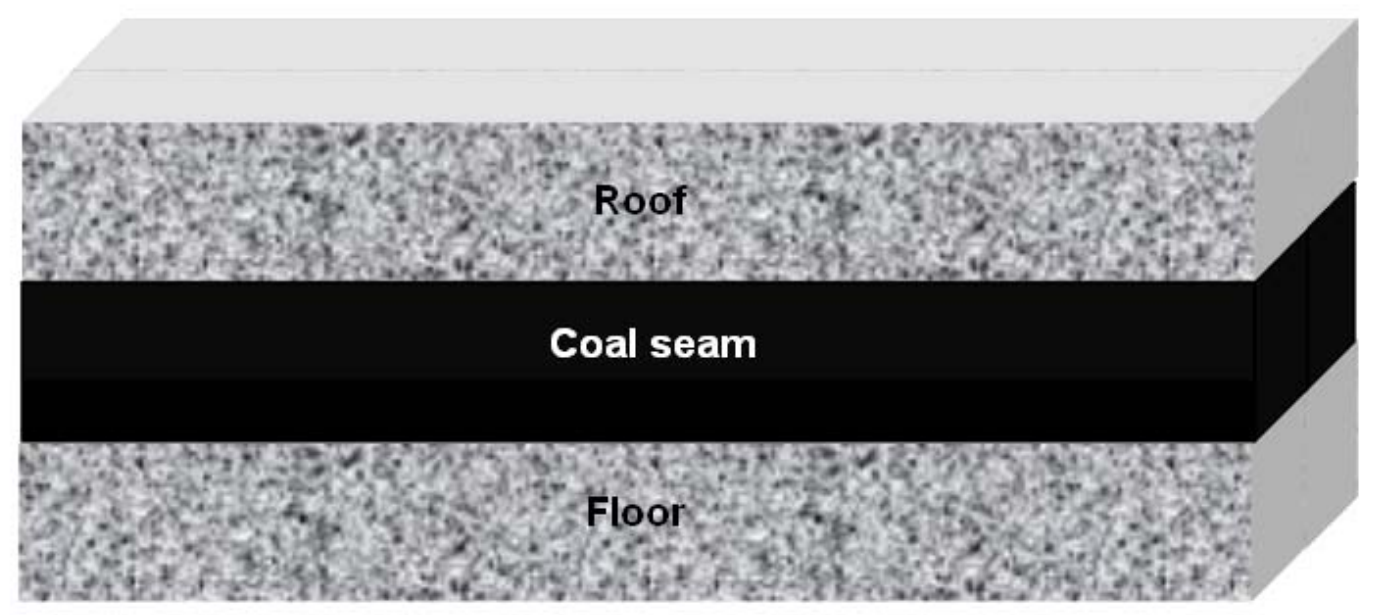

Figure 4.4 The layout of the physical model 
Assuming that the dielectric constant in the coal seam and roof/floor are 3.2 and 6 , respectively, and that the electrical conductivity in the coal seam and roof/floor are 0.0012 $\mathrm{S} / \mathrm{m}$ and $0.1 \mathrm{~S} / \mathrm{m}$, respectively, after the dimension scale factor has been decided, the electrical conductivity scale factor can be determined by $k_{\sigma}=1 / k_{l}$,

For coal: $\sigma_{m}=k_{\sigma} \sigma_{f}=\left(1 / k_{l}\right) \sigma_{f}=5 \times 0.0012=0.006 \mathrm{~S} / \mathrm{m}$

For roof/floor: $\sigma_{m}=k_{\sigma} \sigma_{f}=\left(1 / k_{l}\right) \sigma_{f}=5 \times 0.1=0.5 \mathrm{~S} / \mathrm{m}$

The electrical permeabilities are easily met when all materials in the full-sized system and model are non-magnetics,

$$
\mu_{m}=\mu_{f}
$$

\subsubsection{Selection of Physical Model Materials}

In Section 3.1, the EM properties of the materials with different mixture ratios were tested. From the scale factors analysis in Section 4.2.1, the EM properties of the materials used in the full-sized system and scale physical model is shown in Table 4.1.

Table 4.1 EM properties used in full-sized system and scale physical model

\begin{tabular}{|c|c|c|c|c|}
\hline \multirow{2}{*}{} & \multicolumn{2}{|c|}{ Roof/floor } & \multicolumn{2}{c|}{ Coal seam } \\
\cline { 2 - 5 } & $\begin{array}{c}\text { Full-sized } \\
\text { system }\end{array}$ & $\begin{array}{c}\text { Physical } \\
\text { model }\end{array}$ & $\begin{array}{c}\text { Full-sized } \\
\text { system }\end{array}$ & $\begin{array}{c}\text { Physical } \\
\text { model }\end{array}$ \\
\hline Dielectric Constant & 6 & 6 & 3.2 & 3.2 \\
\hline $\begin{array}{c}\text { Electrical Conductivity } \\
\text { (S/m) }\end{array}$ & 0.1 & 0.5 & 0.0012 & 0.006 \\
\hline
\end{tabular}

From the EM properties testing results and analysis, in order to get the materials with high electrical conductivity to build the roof/floor, salt was added in the mixture. The materials with the mixture ratio of $\mathrm{S}: \mathrm{G}: \mathrm{C}: \mathrm{SA}=7: 0.5: 0.5: 1$ meet both the requirements for the dielectric constant of 6 and the electrical conductivity of 0.5 at a water content of $6.5 \%$ 
(Figure 4.5). For the coal seam, the materials with the ratio of $\mathrm{S}: \mathrm{Co}: \mathrm{C}=5: 5: 1$ meet both of the requirements for the dielectric constant of 3.2 and the electrical conductivity of 0.006 at the water content of $6 \%$ (Figure 4.6). Since the materials with the mixture ratio of $\mathrm{S}: \mathrm{G}: \mathrm{C}: \mathrm{SA}=7: 0.5: 0.5: 1$ also has high electrical conductivity and attenuation rate, it was also used to simulated the geological anomalies in the coal seam.

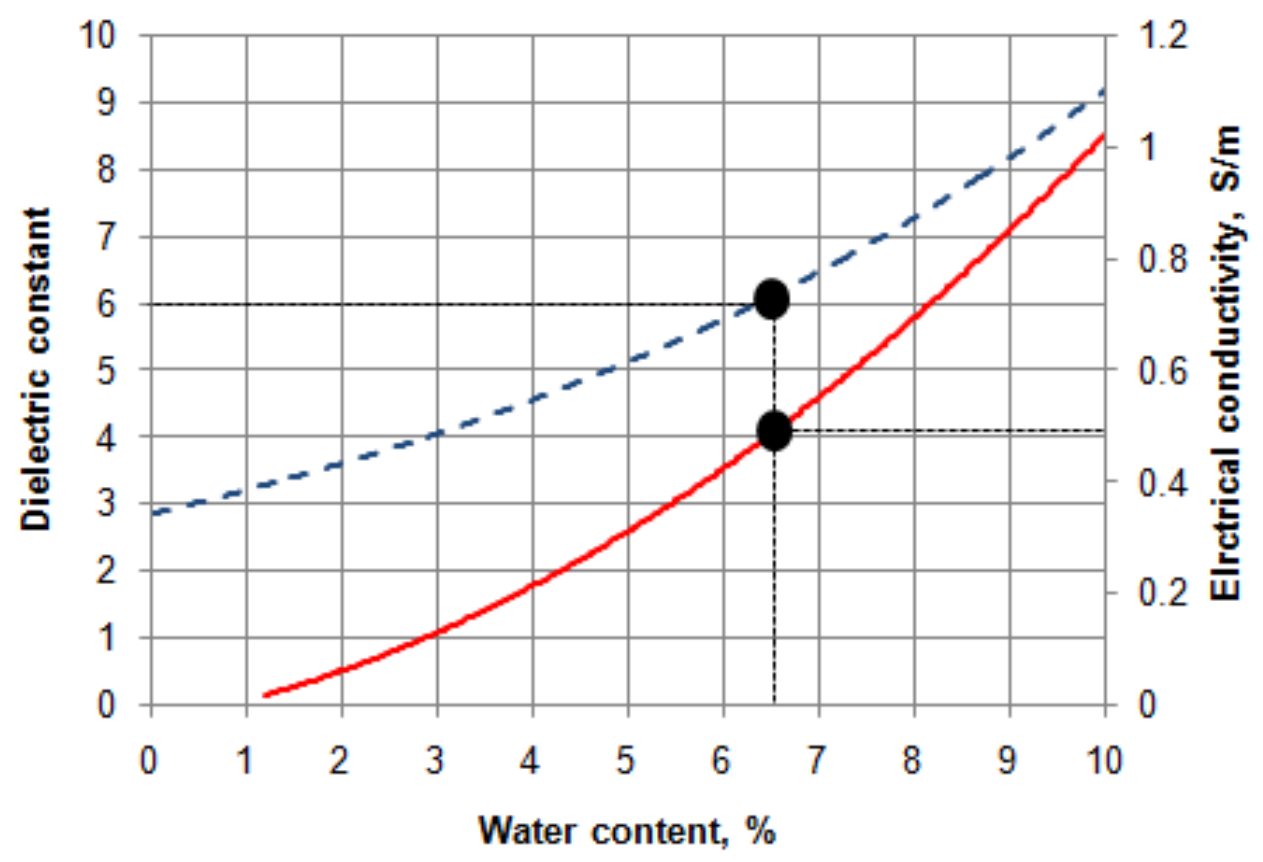

- - - Dielectric constant - Electrical conductivity

Figure 4.5 Materials with mixture ratio of $S: G: C: S A=7: 0.5: 0.5: 1$ with water content at 6.5\% meet the requirement to simulate roof/floor 


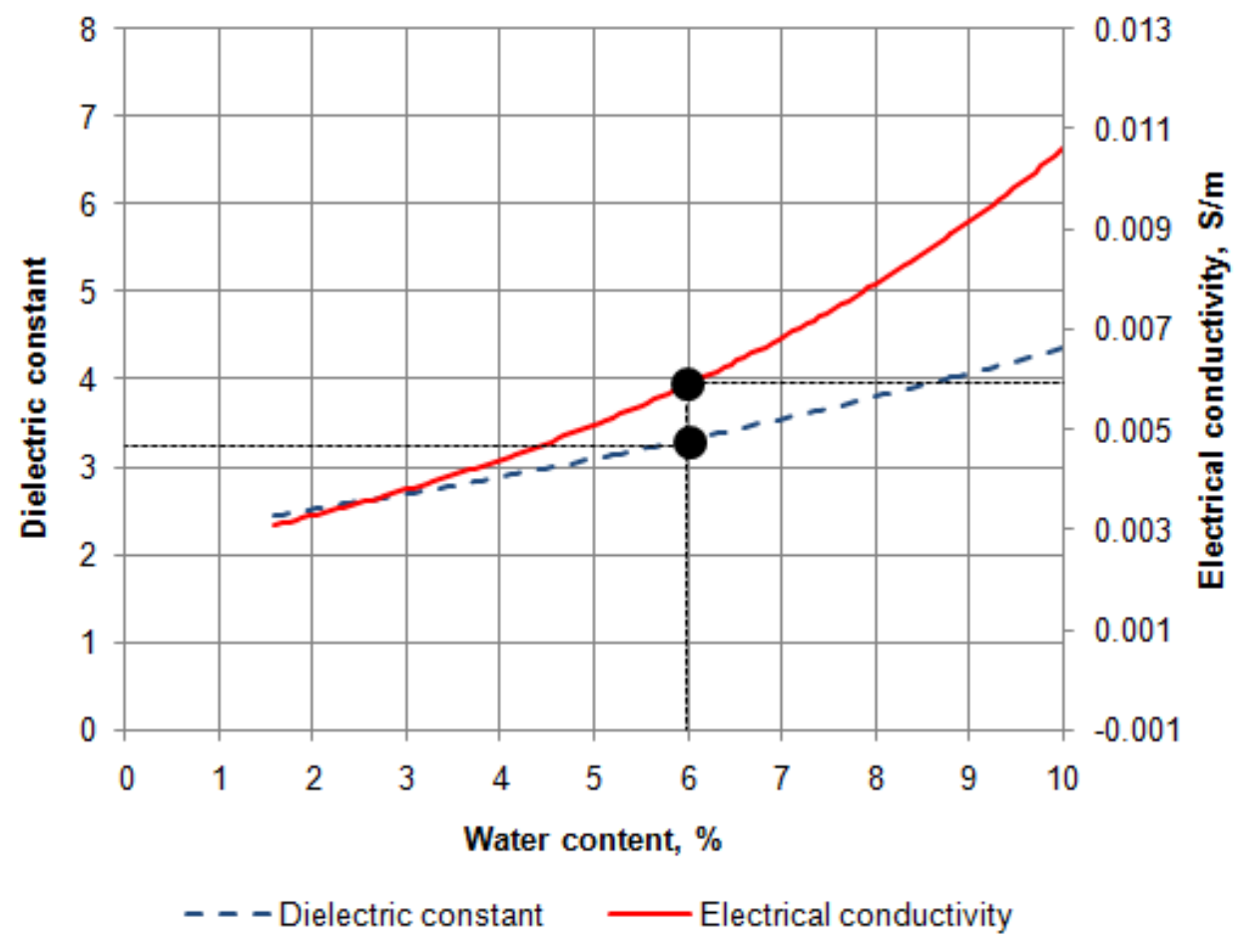

Figure 4.6 Materials with mixture ratio of S:Co:C =5:5:1 with water content at $6 \%$ meet the requirement to simulate coal seam

\subsubsection{Model Design and Construction}

Based on the scale factors and EM properties samples testing discussed in the last section, a scale physical model with roof, floor and coal seam (Figure 4.4) was built. There is 23 " of homogeneous coal seam which will be used for calibration testing. Two sandstone intrusion geological anomalies were constructed (Figure 4.7 and Figure 4.8). Both sandstone intrusions lie vertically at the center of the coal seam. The sandstone intrusion-1 penetrates only half the depth of the coal seam, while sandstone intrusion-2 penetrates the whole coal seam. The material used in the geological anomalies is the same as the roof/floor. The water content was monitored during the entire process of the model construction. The interval for the RIM testing point is $2 \mathrm{in}$.

The physical model survey procedure follows these steps: 
1. Calibration test. A calibration test was conducted along the homogeneous coal seam part to obtain the Standard Attenuation Rate and Transmitter Coupling Factor. These parameters are unique for this physical model and are also very important for RIM tomogram reconstruction.

2. Reconnaissance test. A reconnaissance test was conducted along the enntire coal seam to quickly locate the position of the geological anomalies. A reconnaissance test was also conducted along the roof and the floor. The test results from the roof and the floor can be a reference to compare the signal strength/attenuation rate of the coal seam and the geologic anomalies, which will be helpful to analyze the geological anomalies in the coal seam.

3. RIM test. A RIM test was conducted along the entire coal seam. With the interval of the testing stations at 2 in. and the maximum testing angle of $45^{\circ}$, there were 581 ray paths for a RIM test of the physical model.

4. Data process and analysis. After all the tests have been conducted, the data will be processed and analyzed. The characteristics of the reconstructed RIM attenuation rate tomography tomogram will be compared with the geological characteristics of the known coal seam and the geologic anomalies. 

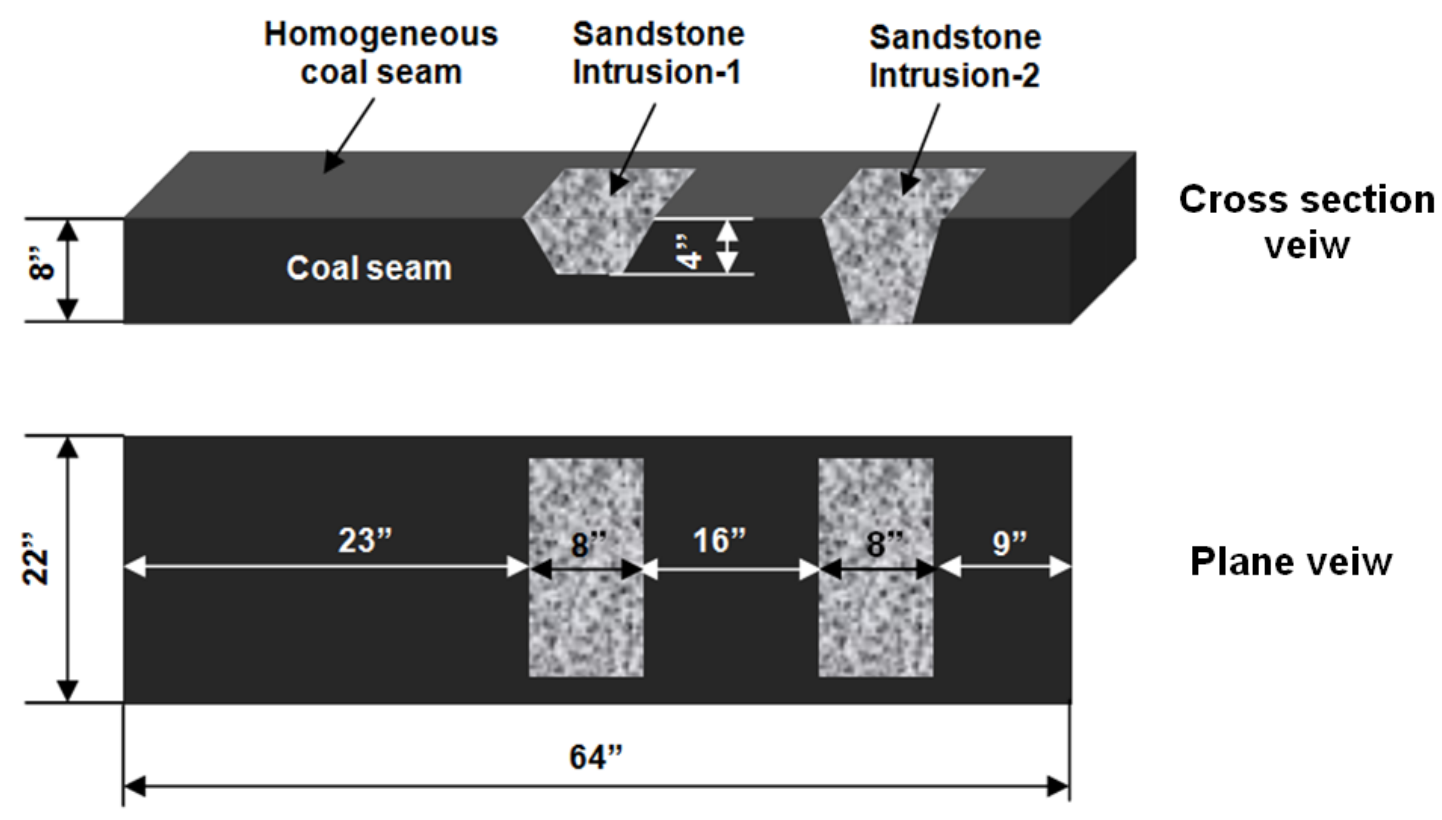

Plane veiw

Figure 4.7 Layout of the geological anomalies in the coal seam

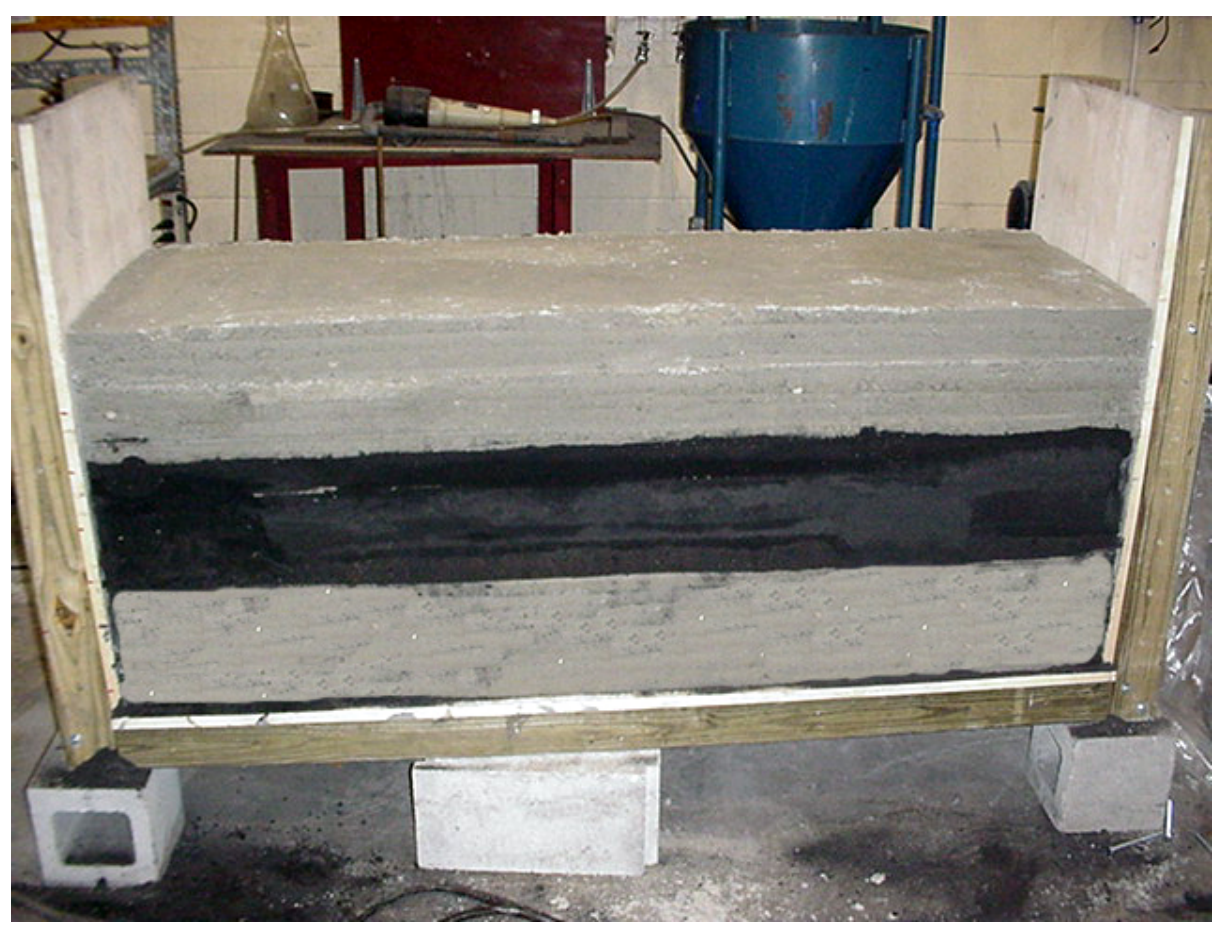

Figure 4.8 Layout of the second scale model 


\subsubsection{Calibration Testing Results}

With this physical model, the calibration test is conducted along the homogeneous coal seam part to obtain the Standard Attenuation Rate (SAR) and Transmitter Coupling Factor (C-Factor) which are unique for this physical model. The transmitter antenna is positioned stationary at one side of the physical model, and the receiver antenna moves from seven different locations at the other side of the physical model with an interval of 2 in. (Figure 4.9).

Because the high frequency radar is used in the physical model testing, and the propagation distance of ray paths is short. The propagation of the EM wave is assumed to be spherical spreading. As discussed in Section 3.5.1, the signal strength can be described by Eq. (3-4) for spherical spreading.

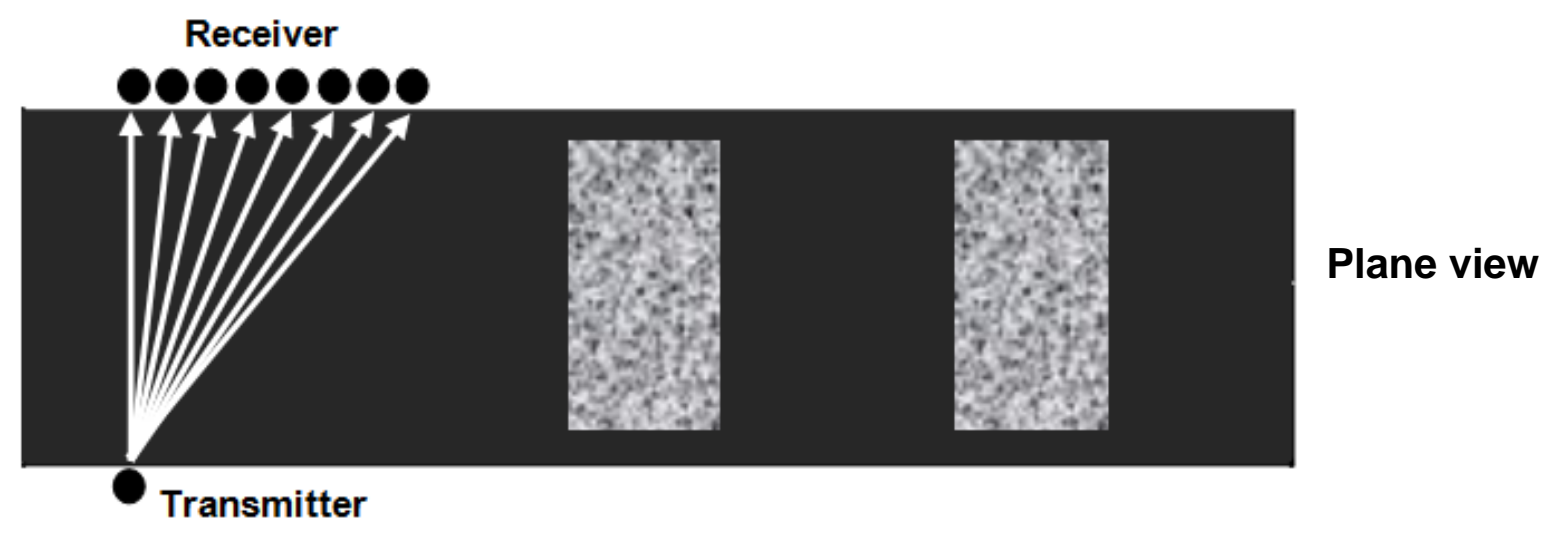

Figure 4.9 Layout of calibration test

According to Eq. (3-7),

$$
20 \log V_{i}+20 \log r_{i}=20 \log V_{0}-20 \log \left(e^{-\alpha r_{i}}\right)
$$

The left side of Eq. (4-1) is called corrected signal strength (CSS) which is obtained from correcting the measured signal strength (MSS) for spherical spreading of the radio 
wave. The CSS is plotted as a function of distance from the transmitter antenna. A linear regression was conducted for this group of data (Figure 4.10). Using this plot, the C-Factor is obtained at the point where $r_{i}=0$. From the calibration test, the standard attenuation rate in the coal seam is about $9.7 \mathrm{~dB} / \mathrm{ft}$, and the $\mathrm{C}$-Factor is about $12.9 \mathrm{~dB}$. Those values are very important for further RIM tomogram processing.

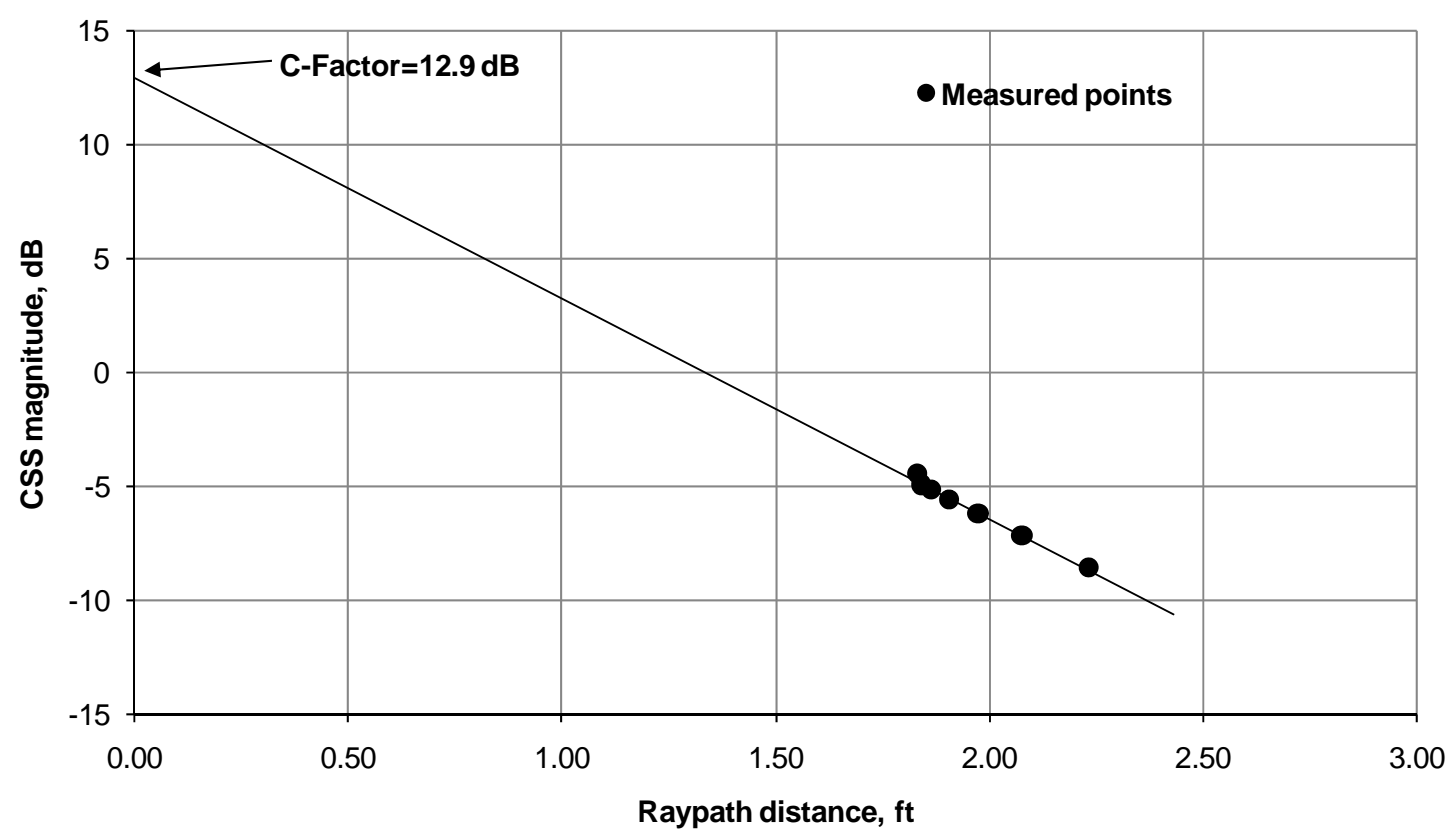

Figure 4.10 Calibration testing result

\subsubsection{Reconnaissance Testing Results}

Reconnaissance testing was conducted along the coal seam, roof, and floor, respectively. As discussed in Section 3.3.2, reconnaissance testing is used to quickly and primarily detect the geological anomalies in the coal seam. The reconnaissance testing in the roof and floor were also conducted because the testing is relatively easy and the test results can be used to compare the test results in the coal seam. Since the signal strength/attenuation rate in the coal seam and roof/floor are totally different, the testing results from those three layers can 
help better understand the signal strength/attenuation rate of the geological anomalies in the coal seam.

All of the three horizontal test lines in the coal seam, roof and floor are in the middle of the seams, and the interval of the testing points is 2 in. (Figure 4.11). In total there were 31 straight ray paths tested for each test line. The signal strength at the three test lines is shown in Figure 4.12. Conversely, the average attenuation rate along the three testing lines is also obtained and is shown in Figure 4.13. The signal strength in the coal seam, especially at the homogeneous part, is much higher than the roof and the floor. This is expected, because the simulation materials in the roof and floor have a higher electrical conductivity and dielectric constant, the attenuation rate is obviously higher than the attenuation rate in the coal seam. There are two obvious signal strength changes along the coal seam, and the signal strength drops sharply at those two places. A contour map is obtained with Surfer (Figure 4.14) which shows the attenuation rate along the coal seam. Compared to the location of the geological anomalies, the locations of the signal changes closely match the location of the geological anomalies in the coal seam. The contour map also shows that the two simulated sandstone intrusions generated different attenuation rates. Sandstone intrusion-1, which just penetrates half of the coal seam, has a smaller attenuation rate as compared to Sandstone intrusion-2 which penetrates completely through the coal seam. That is because there are more EM signals propagating in the coal seam at the location of Sandstone Intrusion-1 than at the location of Sandstone Intrusion-2.

Of course, the reconnaissance test is just a simple test, showing one test line along the coal seam. It cannot fully reflect the location and distribution of the geological anomalies in 
the coal seam. RIM testing must be conducted to obtain more detail information and characteristics of the geological anomalies.

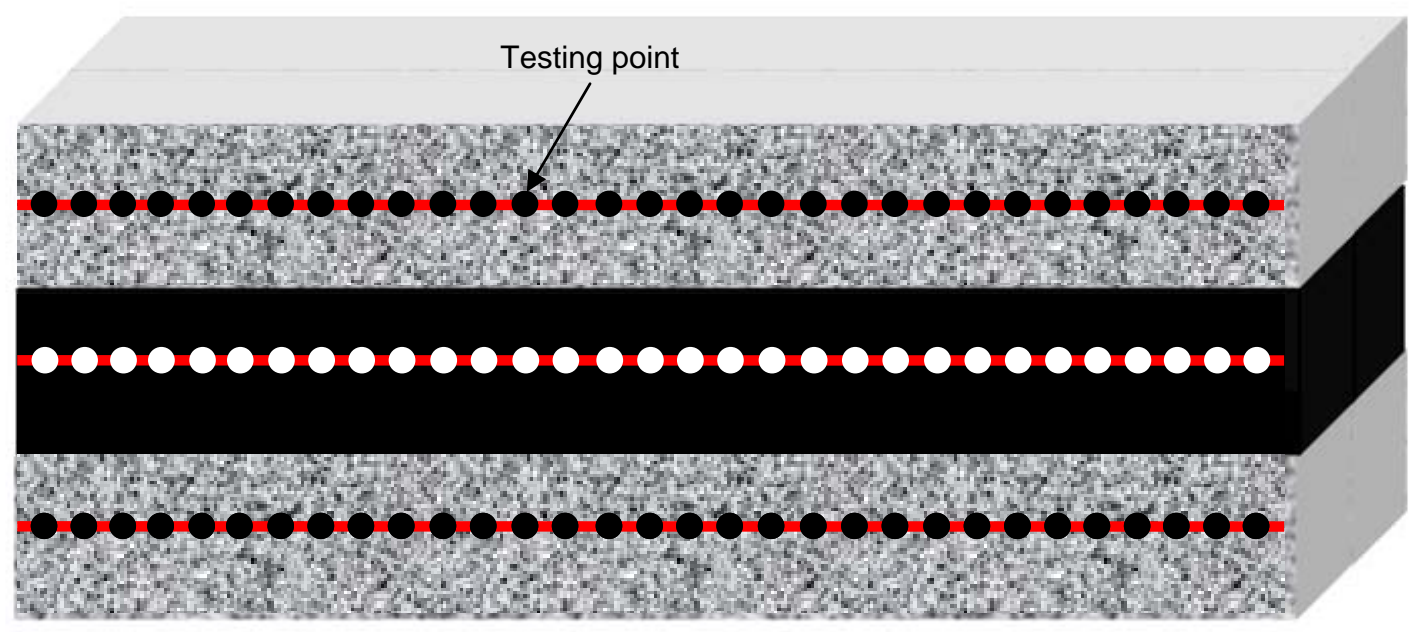

Figure 4.11 The layout of the physical model

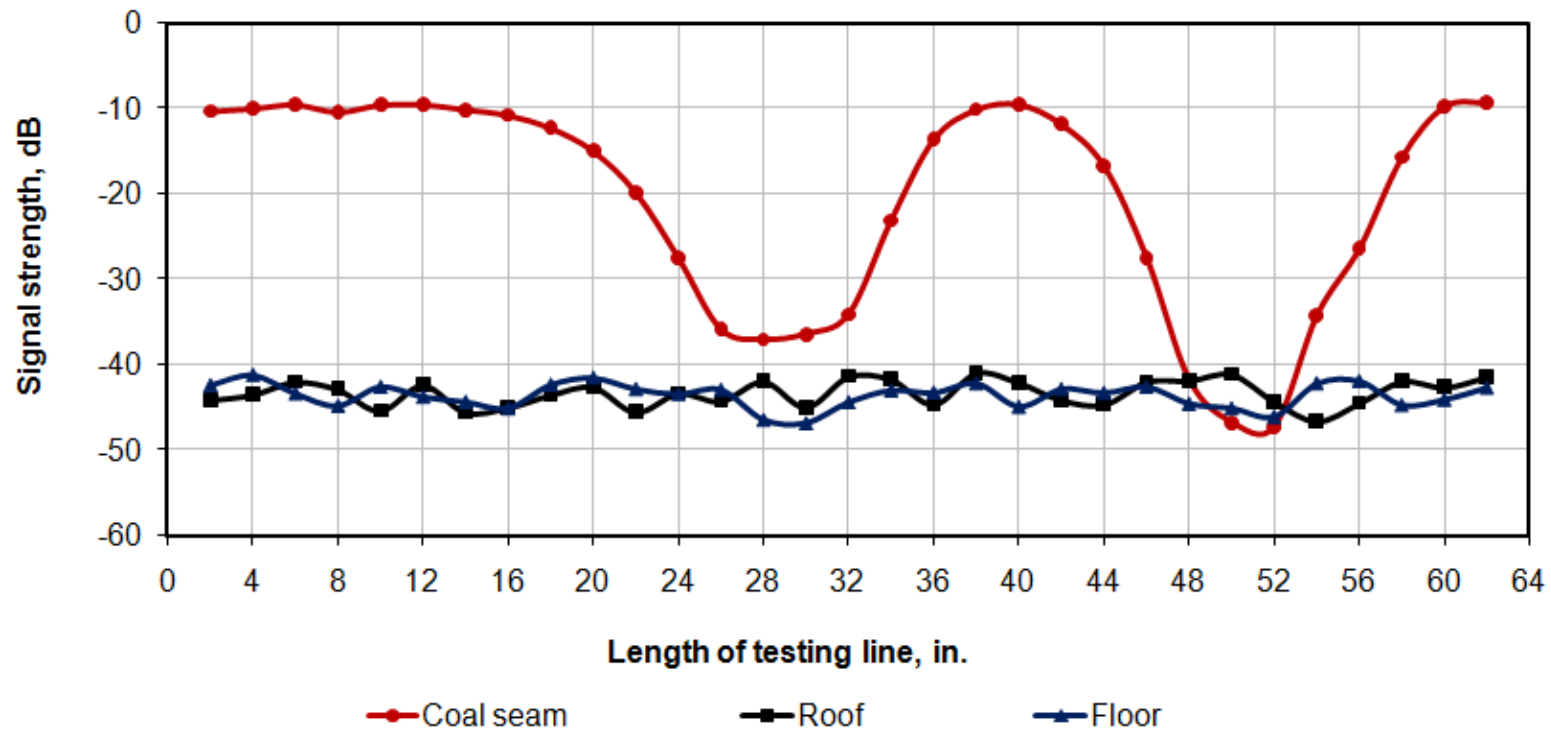

Figure 4.12 Signal strength at the three reconnaissance testing lines of coal seam, roof and floor 


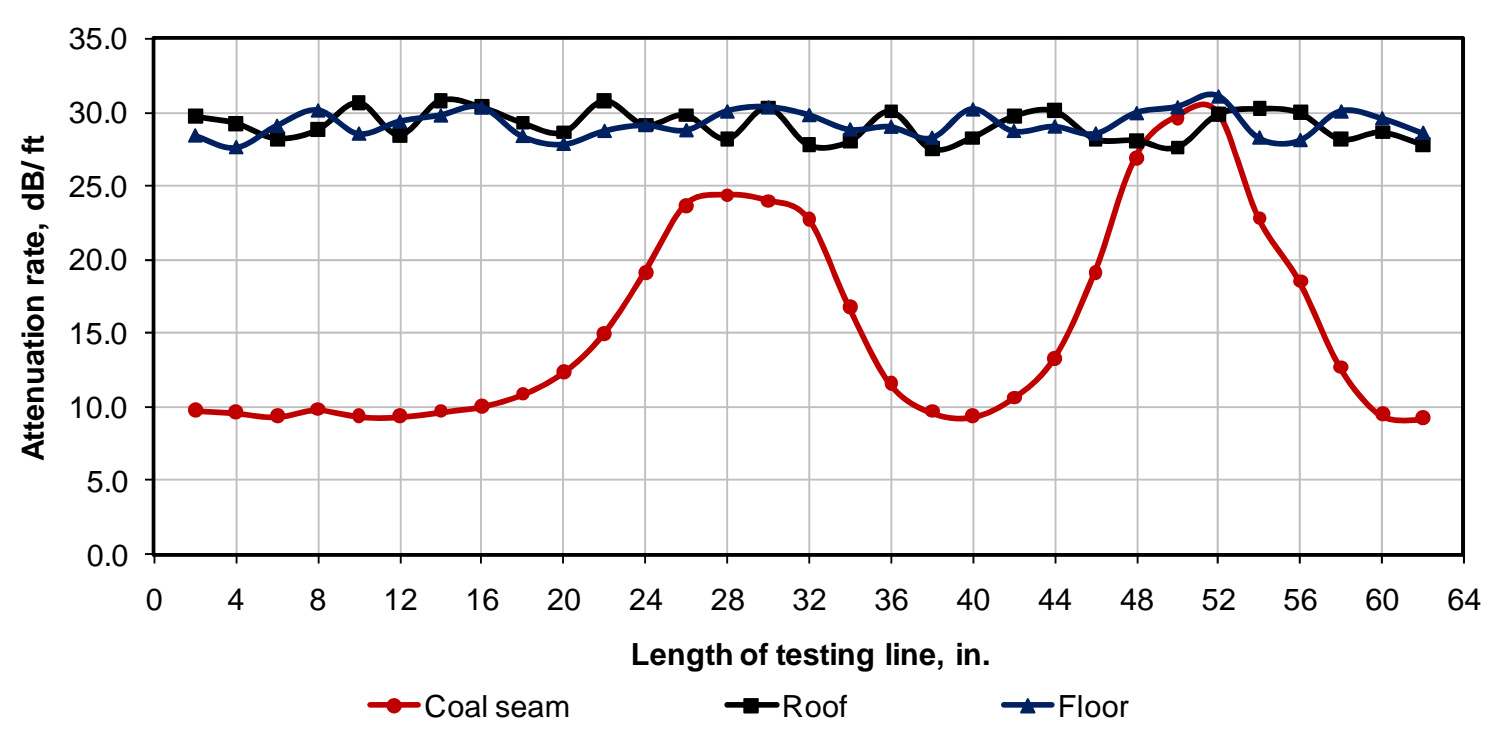

Figure 4.13 Attenuation rate at the three reconnaissance testing lines of coal seam, roof and floor

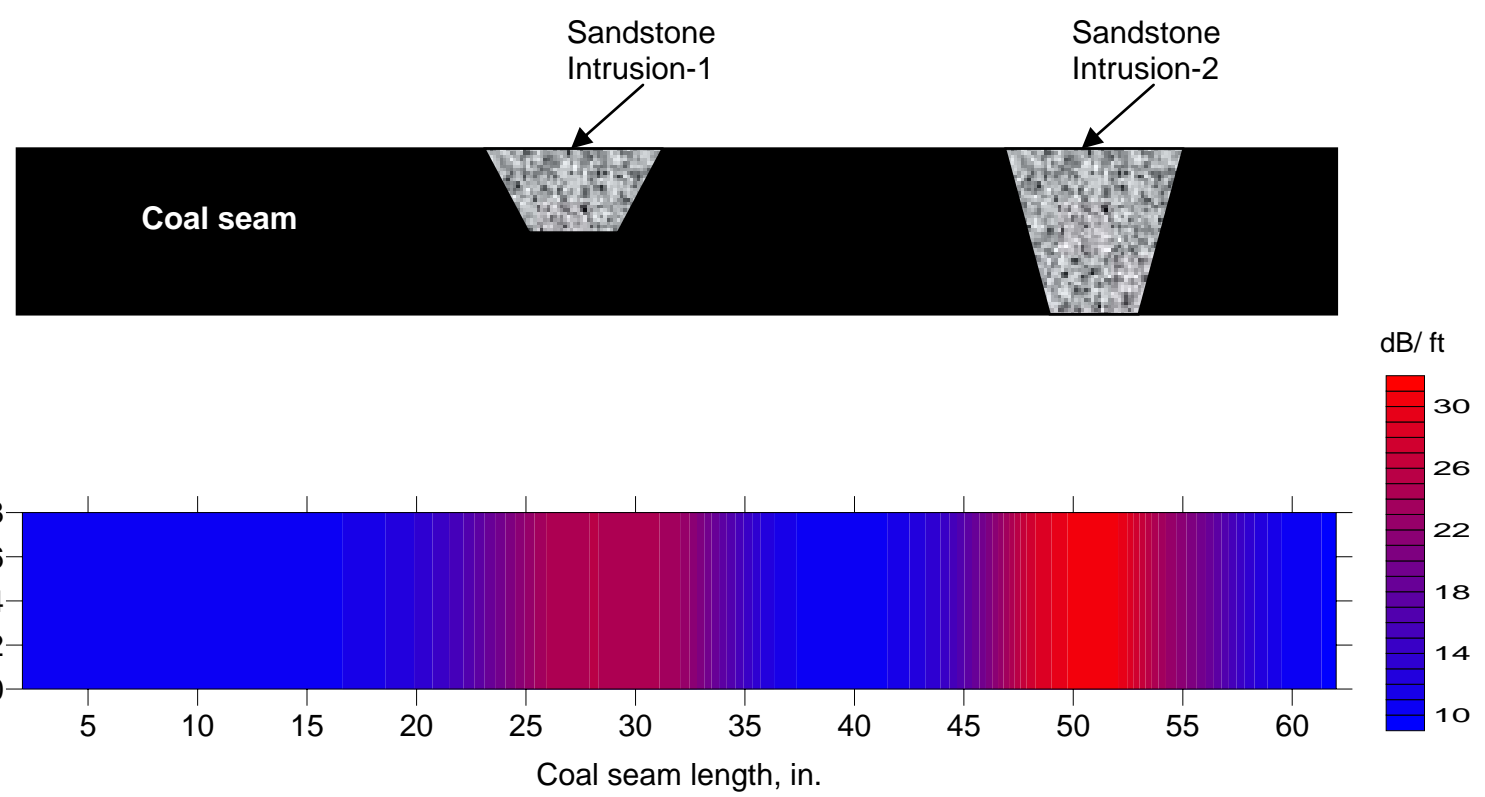

Figure 4.14 Contour of the attenuation rate along the coal seam testing line

\subsubsection{RIM Testing Results}

RIM testing was conducted along the middle of the coal seam. The interval of the testing point is still 2 in. Based on the radar calibration study by Monaghan (2007), the receiver 
antenna will receive signals that shift $90^{\circ}$ from the transmitter antenna. Based on the size of the model and the interval distance, there are a total of 581 ray paths tested along the coal seam. (Figures 4.15 and 4.16). The testing sequence will start from transmitter station No. 1, and then No. 2 , No. 3 , ..., No. 31 . For each transmitter station, there are different numbers of receiver stations, and consequently, there are different numbers of ray paths. For example, 12 ray paths were tested at transmitter station No. 1, 13 ray paths were tested for transmitter station No. 2, and 23 ray paths were tested at transmitter station No. 15, etc.

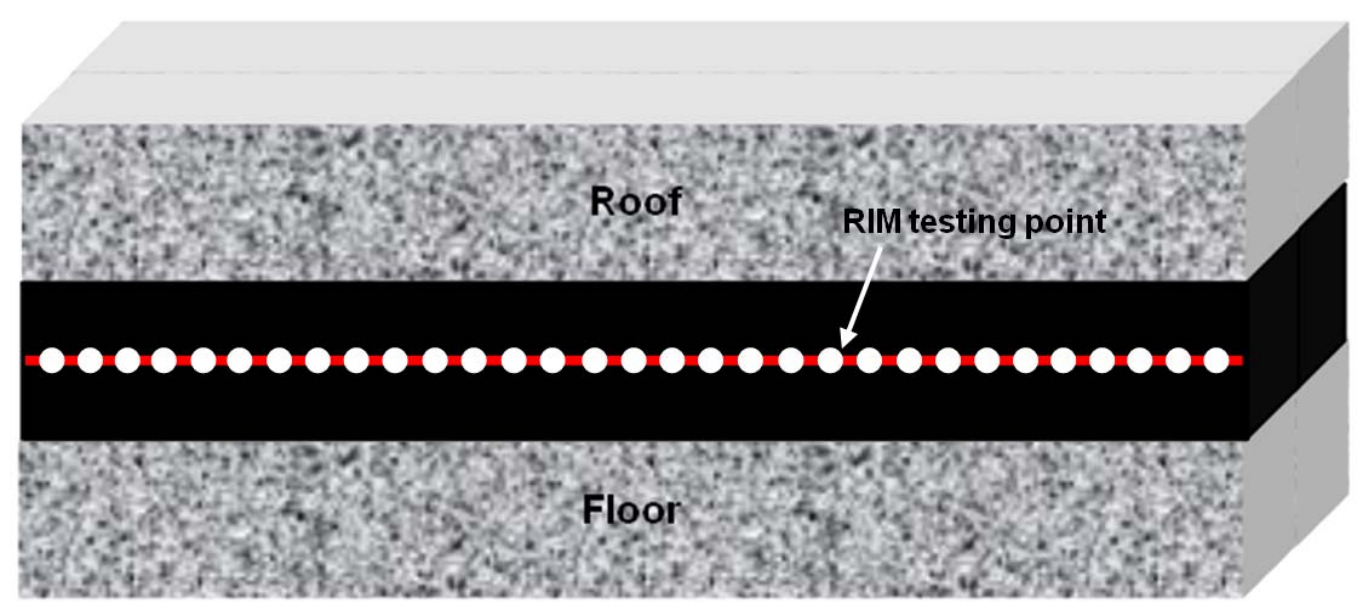

Figure 4.15 RIM testing along the coal seam 
Receiver

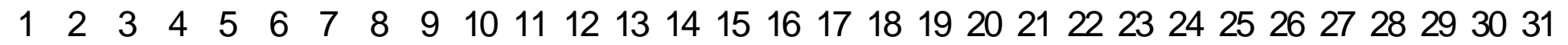

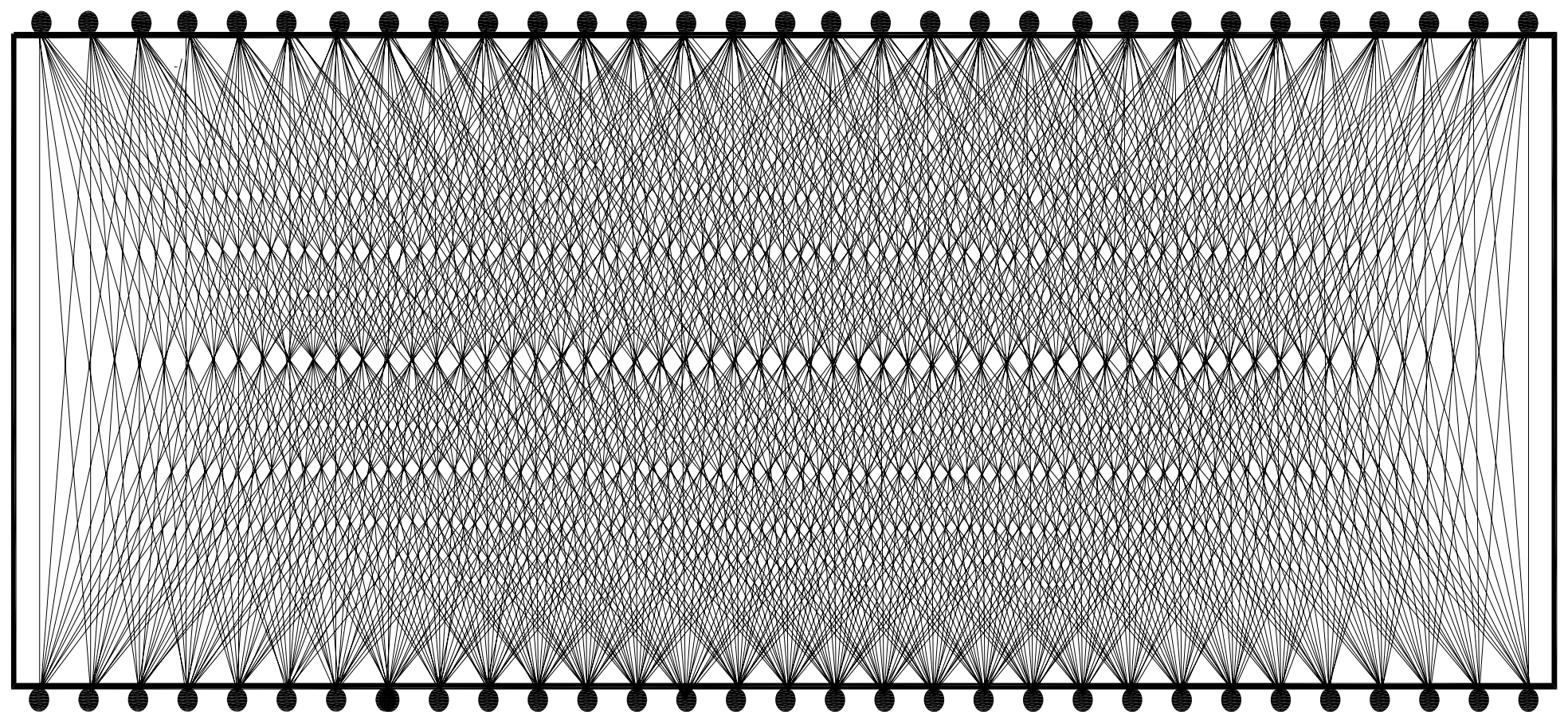

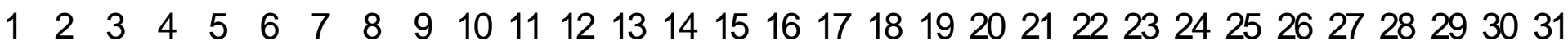
Transmitter

Figure 4.16 Physical model RIM testing scheme and ray paths 
The testing date was processed according to the discussion in Section 3.4. The raw data with format *.DZT was converted to ASCII using a Radan to ASCII conversion utility program RTOAW.EXE. The ASCII data was then imported into a Microsoft Excel spreadsheet to obtain the average peak-to-peak values at a specific signal trace. The average amplitude value of the maximum peak and the minimum peak was acquired. The amplitude value was converted to Volts based on the relationship between amplitude and Volts (Monaghan, 2007). Each of the transmitter stations can be counted as a group of data, and all the data should be processed consecutively from transmitter station No.1 to No.31. So there are total 31 group data and 581 ray paths.

As an example, the signal strength at the transmitter station No. 8 is plotted in Figure 4. 17. 19 receiver stations (from No.1 to No. 19) were tested and signal strengths for the 19 ray paths were obtained. The signal strength is higher at the left side (from station No. 1 to 8) reaching the highest value at the receiver station No. 8 , and then decreasing rapidly at the right side (from station No. 9 to 19). That is because the EM wave propagates in the homogeneous coal seam at the left side, and the signal strength is stronger. Besides, with the distance between the transmitter and the receiver getting closer, the signal strength is getting stronger and reaches the highest value at the shortest distance (both of the transmitter and receiver at the station No. 8). While on the right side, the geological anomaly, Sandstone Intrusion-1, has a higher attenuation rate than the coal seam, so the signal strength at the right side is smaller than the left side. 


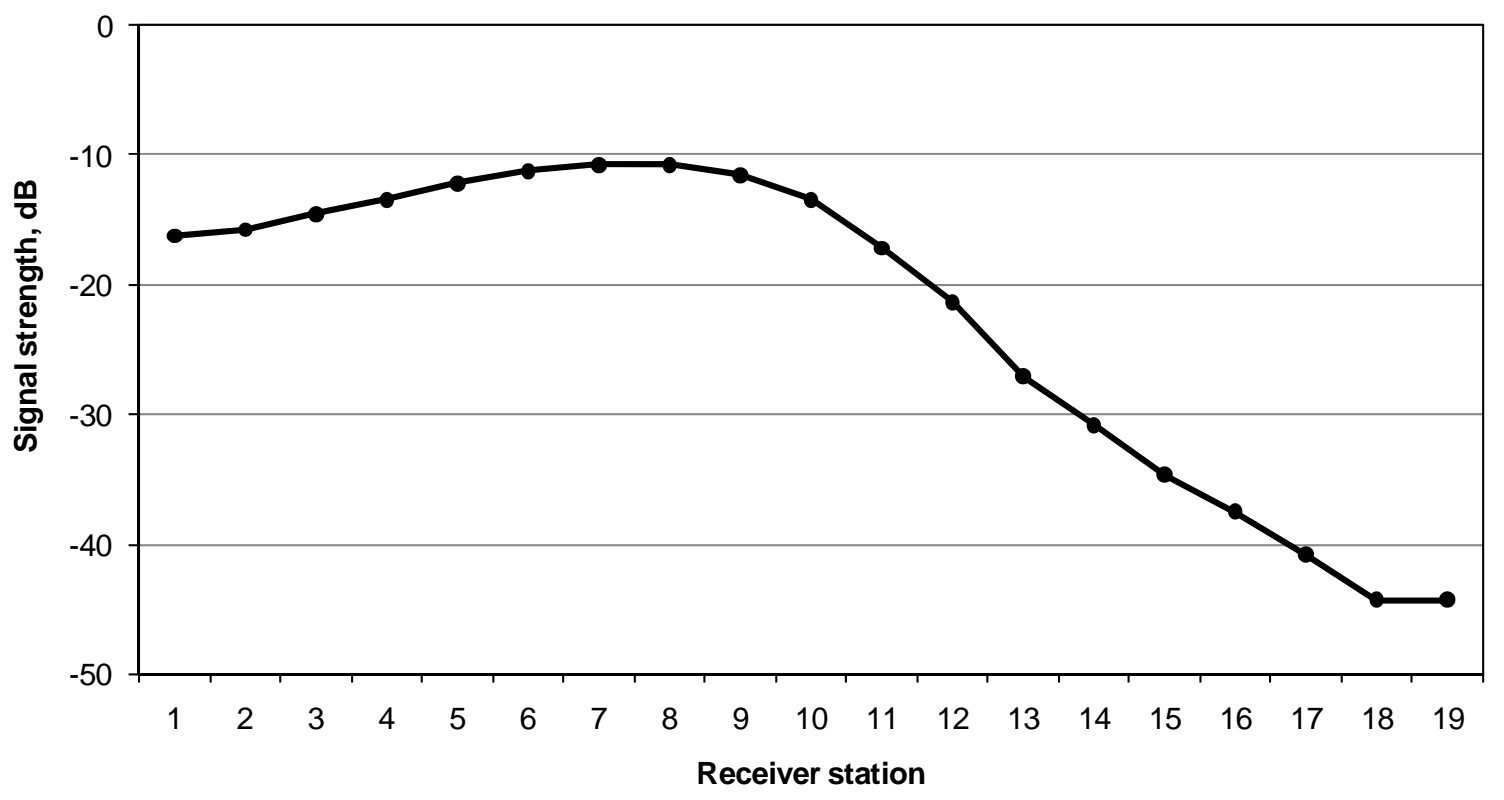

Figure 4.17 Signal strength at different receiver stations (transmitter station No.8)

In order to reconstruct the attenuation tomogram, the testing area was divided into $2 \times 1$ in. cell. The size of the physical model is $62 \times 22$ in., but the transmitter/receiver station starts 2 in. away from the edge. So the actual size used in the software to reconstruct the tomogram is $62 \times 22$ in., and there are a total of $31 \times 22=682$ cells (Figure 4.18 ). The index of the cell is also shown in Figure 4. 18.

Before processing the RIM testing data and reconstructing the attenuation tomogram, a forward model was analyzed. The forward model had the same size and anomaly structures as the laboratory physical model. The attenuation rate which was assigned to the forward model was roughly selected according to the results of reconnaissance testing. So the average attenuations rate in the coal seam, sandstone intrusion-1, and sandstone intrusion-2 are $9.7 \mathrm{~dB} / \mathrm{ft}, 24 \mathrm{~dB} / \mathrm{ft}$, and $27 \mathrm{~dB} / \mathrm{ft}$, respectively (Figure 4.19). The forward modeling result (Figure 4.20) shows that the coal seam and the geological anomalies are 
well reconstructed. The characteristics of anomalies location, shape, and attenuation closely match the characteristics of the embedded physical model. 


\section{Receiver stations}

\begin{tabular}{|c|c|c|c|c|c|c|c|c|c|c|c|c|c|c|c|c|c|c|c|c|c|c|c|c|c|c|c|c|c|c|}
\hline 52 & & & & & & & & & & & & & & & & 68 & 69 & 70 & 71 & 72 & 673 & 674 & 75 & 676 & 677 & 678 & 579 & 680 & 581 & \\
\hline 21 & & & & & & & & & & & & & & & & & 38 & & & & 42 & 43 & & 45 & 646 & 647 & 548 & 49 & & \\
\hline$\overline{0}$ & & & & & & & & & & & & & & & & & 77 & 88 & & & & 12 & 13 & 14 & 15 & 16 & 17 & 18 & 19 & \\
\hline & 0 & & & & & & & & & & & & & & & & & 77 & & & & 81 & & & & & & & & \\
\hline 28 & 99 & 0 & 31 & 32 & 33 & 34 & 35 & 536 & 37 & 38 & 39 & 40 & 11 & 42 & $\overline{43}$ & 544 & 545 & 46 & 47 & 48 & 49 & 50 & 51 & 552 & 553 & 154 & 555 & 56 & 57 & \\
\hline 7 & & & & 1 & 2 & 03 & 504 & 505 & 06 & 77 & & & & & 512 & 513 & 514 & 15 & 16 & 17 & 518 & 519 & 520 & 521 & 522 & 523 & 524 & 25 & 26 & \\
\hline & & & & & & & 473 & 74 & & & & & & & & 482 & 83 & 84 & 85 & 86 & 37 & 488 & 89 & 490 & 491 & 92 & 93 & 94 & 95 & \\
\hline$\overline{55}$ & 36 & 37 & 88 & 39 & 40 & 41 & 442 & 443 & 44 & 45 & 46 & 47 & 448 & 449 & 450 & 451 & 452 & 453 & 454 & 455 & 456 & 457 & 458 & 459 & 460 & 461 & 462 & 463 & 464 & 6 \\
\hline$\overline{24}$ & 5 & 76 & 07 & 408 & 409 & 410 & 411 & 412 & 413 & 14 & 15 & 416 & 417 & 418 & 419 & 420 & 421 & 422 & 423 & 424 & 425 & 426 & 427 & 428 & 429 & 430 & 431 & 432 & 433 & 13 \\
\hline 73 & 74 & 5 & & 77 & 378 & 379 & 380 & 381 & 82 & 83 & & & 386 & 387 & 388 & 389 & 390 & 391 & 392 & 393 & 394 & 395 & 396 & 397 & 398 & 399 & 400 & 401 & 402 & 40 \\
\hline 2 & 3 & 4 & & & 4 & 348 & 349 & 350 & ? & 22 & & & & & & 358 & 359 & 60 & 61 & 362 & 363 & 364 & 365 & 366 & 367 & 368 & 369 & 370 & 871 & 37 \\
\hline 11 & 12 & 13 & 14 & 315 & 316 & 317 & 318 & 319 & 320 & 21 & 22 & 323 & 324 & 325 & 326 & 327 & 328 & 329 & 330 & 331 & 332 & 333 & 334 & 335 & 336 & 337 & 338 & 339 & 340 & 34 \\
\hline 30 & 1 & 32 & 33 & 84 & 285 & 286 & 287 & 288 & 89 & 90 & 91 & 92 & 93 & 294 & 295 & 296 & 297 & 98 & 0 & 300 & 01 & 302 & 03 & 304 & 305 & 306 & 307 & 308 & 09 & 310 \\
\hline 49 & 50 & 51 & 52 & 253 & 254 & 255 & 256 & 257 & 58 & 59 & & 261 & 262 & 263 & 264 & 265 & 266 & 267 & 268 & 269 & 270 & 271 & 272 & 273 & 274 & 275 & 276 & 277 & 278 & 27 \\
\hline 18 & 19 & 20 & 21 & 22 & 223 & 224 & 225 & 226 & 27 & 28 & & & 231 & 232 & 233 & 234 & 235 & 236 & 237 & 238 & 239 & 240 & 241 & 242 & 243 & 244 & 245 & 246 & 247 & 24 \\
\hline 87 & 88 & 39 & & 191 & 192 & 193 & 194 & 195 & 196 & 197 & 198 & 199 & 200 & 201 & 202 & 203 & 204 & 205 & 206 & 207 & 208 & 209 & 210 & 211 & 212 & 213 & 214 & 215 & 216 & 217 \\
\hline 156 & 157 & 158 & 159 & 160 & 161 & 162 & 163 & 164 & 165 & 66 & 67 & 168 & 169 & 170 & 171 & 172 & 173 & 174 & 17 & 176 & 177 & 178 & 179 & 180 & 181 & 182 & 3 & 184 & 185 & 18 \\
\hline 125 & 126 & 127 & 128 & 129 & 130 & 131 & 132 & 133 & 134 & 35 & 136 & 137 & 138 & 139 & 140 & 141 & 142 & 143 & 144 & 145 & 146 & 147 & 148 & 149 & 150 & 151 & 152 & 153 & 154 & 15 \\
\hline 94 & 95 & 96 & 97 & 98 & 99 & 100 & 101 & 102 & 103 & 104 & 105 & 106 & 107 & 108 & 109 & 110 & 111 & 112 & 113 & 114 & 115 & 116 & 117 & 118 & 119 & 120 & 121 & 122 & 123 & 124 \\
\hline 63 & 64 & 65 & 66 & 67 & 68 & 69 & 70 & 71 & 72 & 73 & 74 & 75 & 76 & 77 & 78 & 79 & 80 & 81 & \begin{tabular}{|l|}
82 \\
\end{tabular} & 83 & 84 & 85 & 86 & 87 & 88 & 89 & 90 & 91 & 92 & 93 \\
\hline 32 & 33 & 34 & 35 & 36 & 37 & 38 & 39 & 40 & 41 & $\overline{42}$ & $\overline{43}$ & $\overrightarrow{44}$ & 45 & 46 & 47 & 48 & 49 & 50 & 51 & 52 & 53 & 54 & 55 & 56 & 57 & 58 & 59 & 60 & 61 & 62 \\
\hline 1 & 2 & 3 & 4 & 5 & 6 & 7 & 8 & 9 & 10 & 11 & 12 & 13 & 14 & 15 & 16 & 17 & 18 & 19 & 20 & & 22 & 23 & 24 & 25 & 26 & 27 & 28 & 29 & 30 & 3 \\
\hline
\end{tabular}

\section{Transmitter stations}

Figure 4.18 Divided cells and the index of cells for the physical model 


\section{Receiver stations}

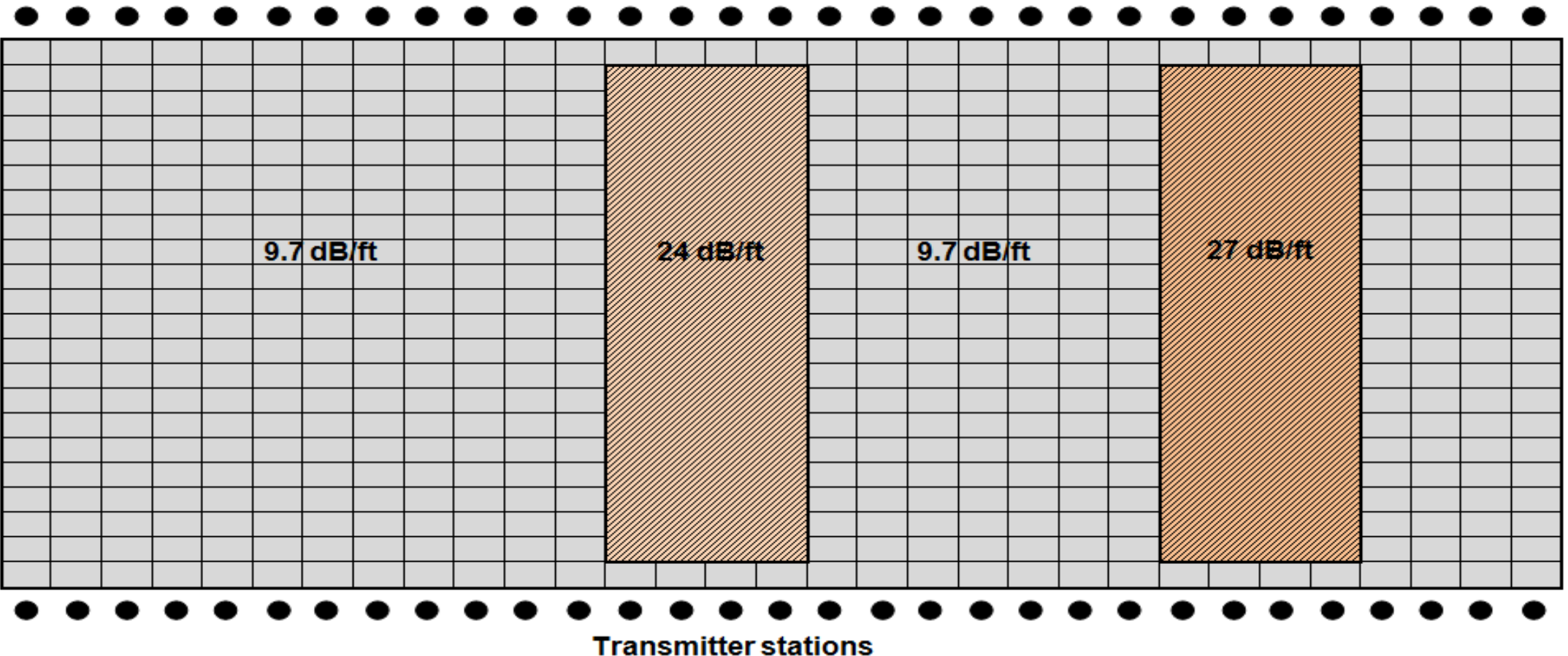

Figure 4.19 Layout of forward model which has the same size and anomaly structures as physical model 


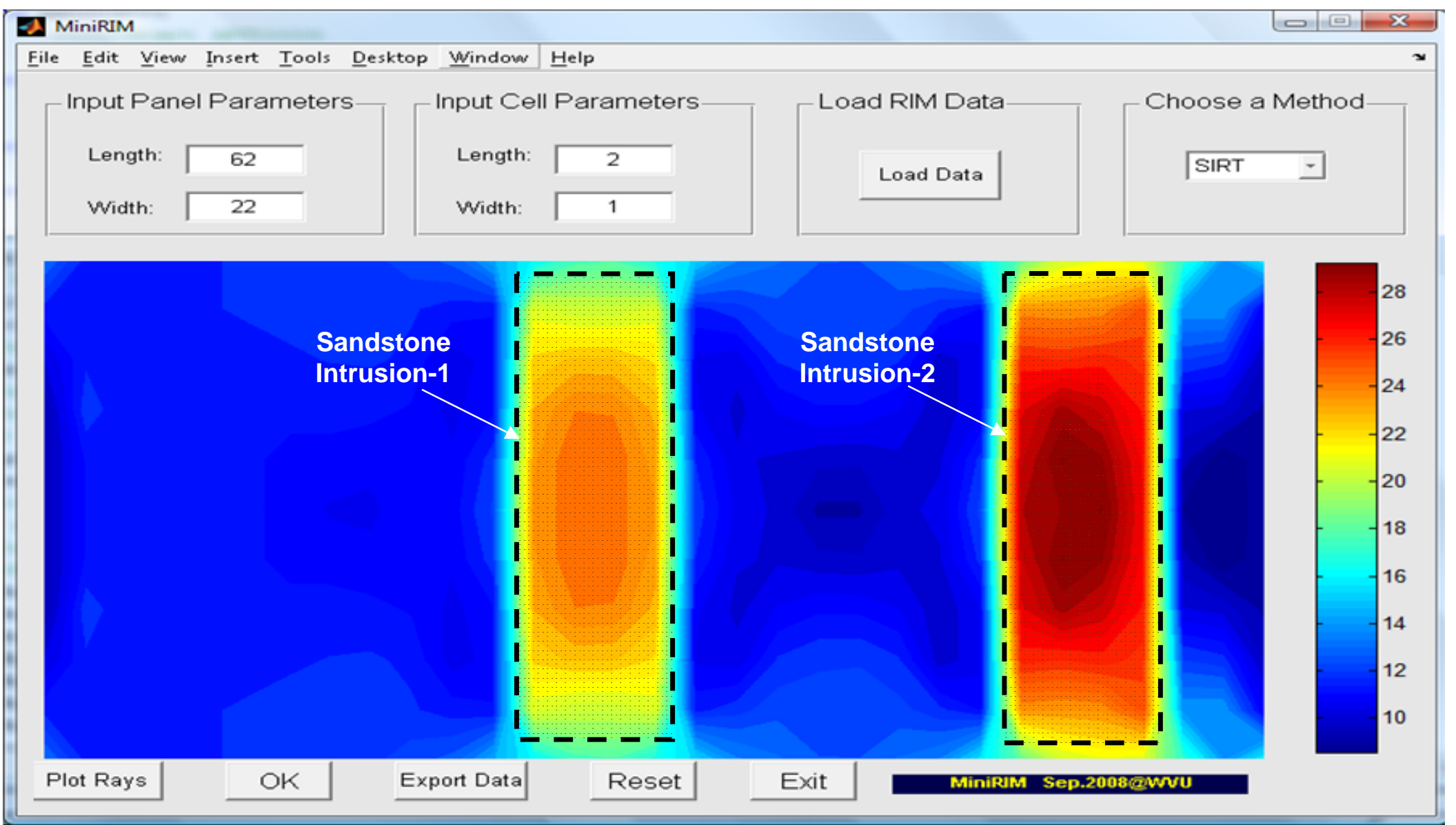

Figure 4.20 Forward modeling result 
In the data process of the physical model RIM testing, the distance which penetrates each cell can be calculated according to the location of the testing station and the cell size. For the input data $Y=V_{0}-V_{i}-20 \log \left(r_{i}\right)$, all the parameters including $V_{o}, V_{i}$, and $r_{i}$ can be obtained for a specific ray path. The RIM testing attenuation rate tomogram (Figure 4. 21) was obtained with the MiniRIM tomogram software.

From the RIM attenuation rate tomogram, the locations and shapes of the anomalies closely matched the embedded physical geological anomalies (Figure 4.5) except the four edges ( 1 in. gap) (Figure 4.22). That is because the attenuation caused by the 1 in. of coal seam at the edge that has little or no influence on the total attenuation caused by the sandstone intrusion geological anomalies. The attenuation rate exported from the software is shown in Figure 4.23. The average attenuation rate obtained from the tomogram in the coal seam, Sandstone Intrusion-1 and Sandstone Intrusion-2 are about $12.7 \mathrm{~dB} / \mathrm{ft}, 24.1 \mathrm{~dB} / \mathrm{ft}$, and $26.3 \mathrm{~dB} / \mathrm{ft}$, respectively. The relative error of the attenuation rate is shown in Figure 4.24. The average relative error of attenuation rate in the Sandstone Intrusion-1 and Sandstone Intrusion-2 are about 5.3\%, and 9.2\%, respectively. So, the attenuation rate of the geological anomalies is well reconstructed.

From the reconstructed attenuation rate tomogram and the exported data analysis, most of the results match the characteristic of the embedded physical geological anomalies. That is to say, the simulation materials used in the physical model can be used to simulate the coal seam, roof, and floor which have different electromagnetic characteristics (dielectric constant and electric conductivity). Besides, the developed RIM software is capable of processing the RIM testing data in the laboratory and reconstructing the attenuation tomogram. 


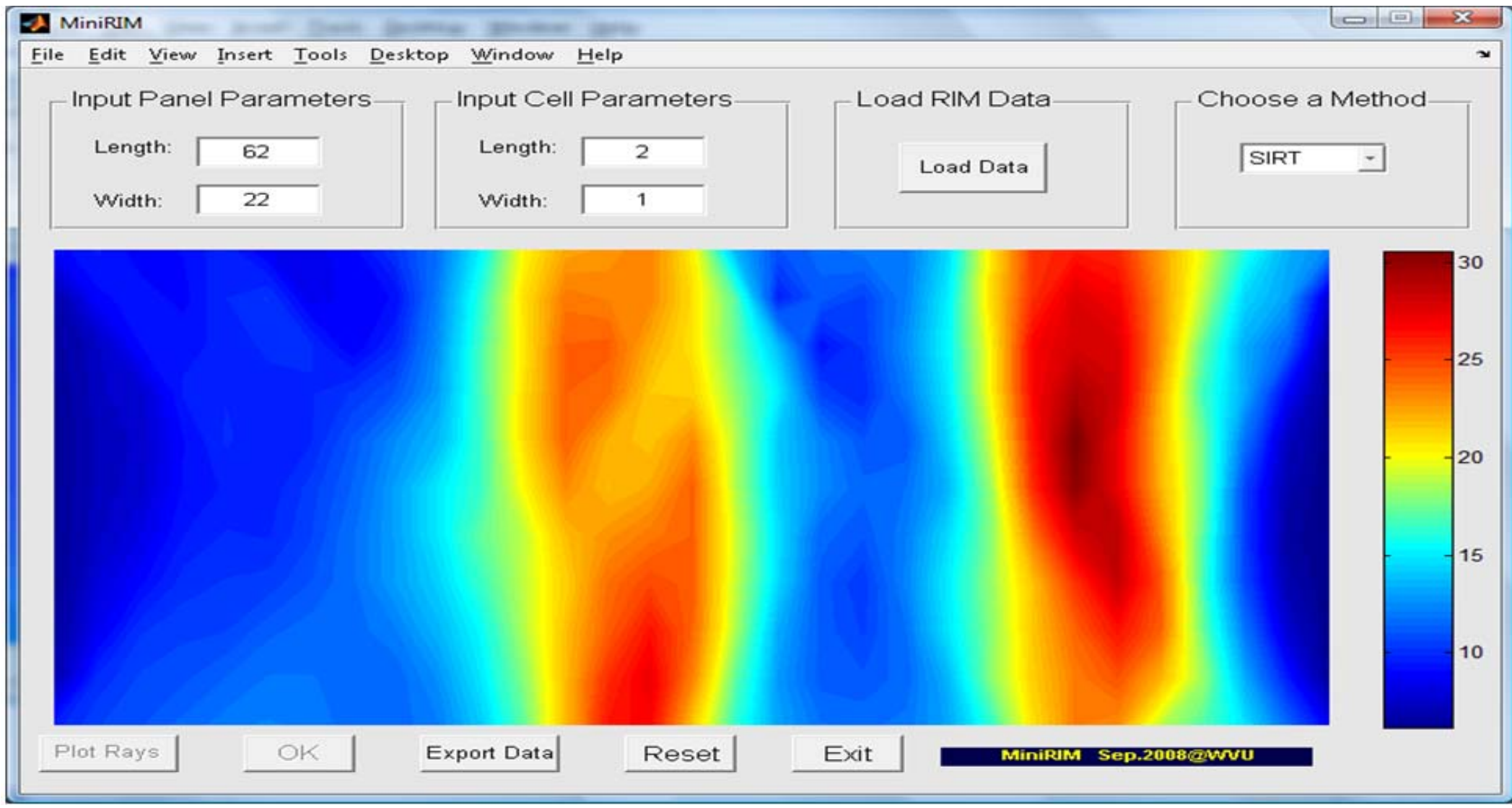

Figure 4.21 RIM testing attenuation rate tomogram 


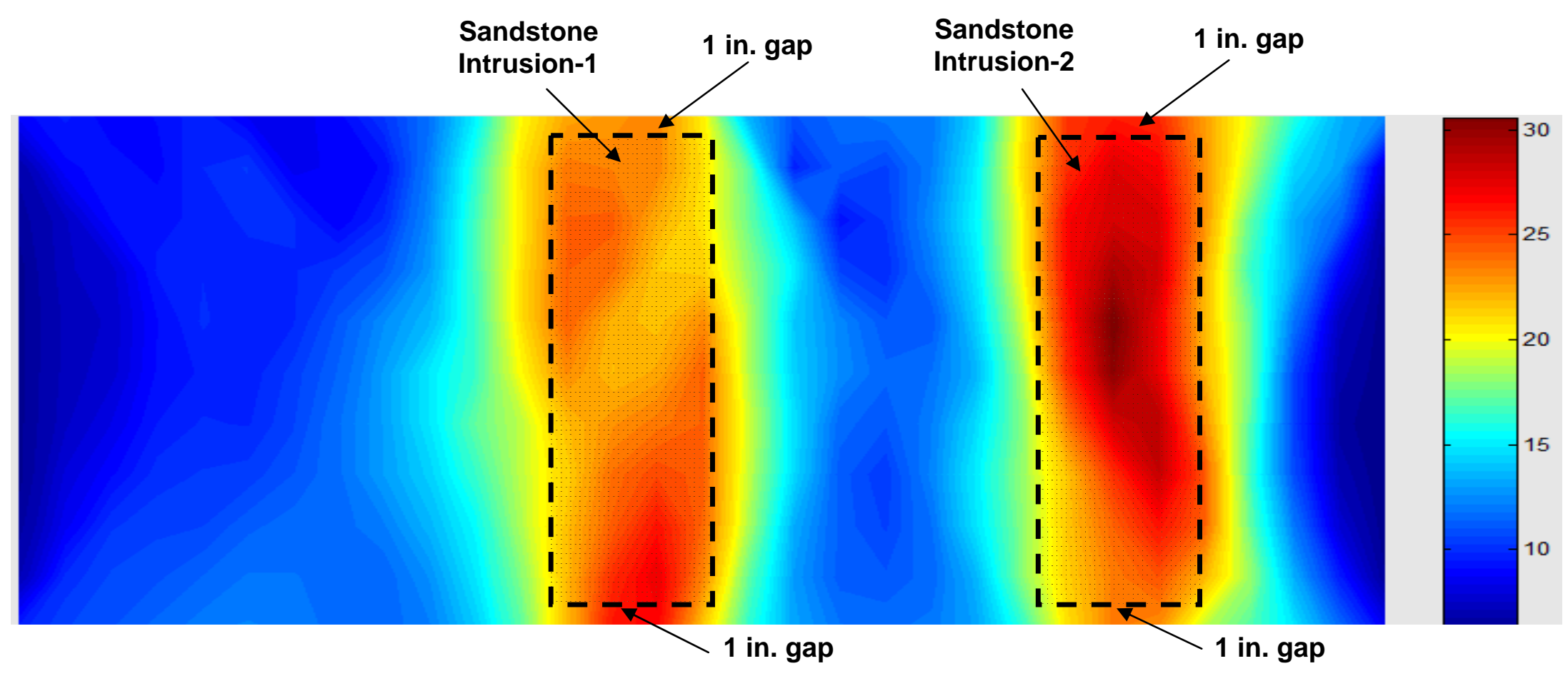

Figure 4.22 Comparison of the embedded geological anomalies and the reconstructed attenuation rate tomogram 


\section{Sandstone}

Intrusion-1

\section{Sandstone}

Intrusion-2

\begin{tabular}{|c|c|c|c|c|c|c|c|c|c|c|c|c|c|c|c|c|c|c|c|c|c|c|c|c|c|c|c|c|c|c|}
\hline 10.3 & 10.6 & 11.2 & 11.8 & 12.5 & 12.5 & 12.3 & 12.0 & 12.0 & 12.7 & 14.4 & $17 . \lambda$ & 22.3 & 27.1 & 27.4 & 21.8 & 16.8 & 13.7 & 12.5 & 12.1 & 12.6 & 13.4 & 16.5 & 21.4 & 24.2 & 23.8 & 19.7 & \begin{tabular}{|l}
17.1 \\
\end{tabular} & 15.3 & 12.6 & 9.5 \\
\hline 9.0 & 10.3 & 10.7 & 1.2 & 11.9 & 2.3 & 12.6 & 2.4 & 12.3 & 12.9 & 15.1 & 17.7 & 22.3 & 6.4 & 27.0 & 22.6 & 17.4 & 14.1 & 2.9 & 13.0 & 13.1 & 14.2 & 17.3 & 21.6 & 24.0 & 23.5 & 19.7 & 18.1 & 14.8 & 11.2 & 8.3 \\
\hline 7.6 & 10.3 & 10.7 & 11.0 & 11.6 & 12.2 & 12.2 & 12.1 & 2.1 & 3.0 & 15.3 & 18.0 & 22.3 & 26.4 & 28.0 & 23.5 & 17.7 & 13.7 & 11.9 & 11.9 & 12.1 & 14.2 & 18.4 & 22.1 & 24.2 & 25.3 & 22.2 & 18.6 & 14.3 & 10.4 & 6.7 \\
\hline 7.0 & 9.6 & 10.6 & 10.9 & 11.4 & 11.9 & 12.0 & 12.1 & 12.3 & 13.5 & 15.3 & 18.7 & 22.5 & 25.8 & 27.9 & 24.4 & 18.1 & 13.6 & 11.6 & 11.4 & 12.4 & 14.5 & 18.7 & 21.9 & 24.3 & 25.9 & 23.5 & 19.0 & 13.3 & 9.4 & 6.2 \\
\hline 6.7 & 8.9 & 10.6 & 10.7 & 10.9 & 11.6 & 12.1 & 12.0 & 12.2 & 13.9 & 15.7 & 18.9 & 22.4 & 25.4 & 27.5 & 24.5 & 19.0 & 13.6 & 11.6 & 11.1 & 12.4 & 15.2 & 18.3 & 22.3 & 25.3 & 26.8 & 24.4 & 18.6 & 12.8 & 8.5 & 6.1 \\
\hline 6.5 & 8.6 & 10.0 & 10.6 & 10.7 & 11.3 & 11.6 & 12.0 & 13.0 & 14.1 & 16.0 & 19.2 & 22.4 & 24.9 & 26.9 & 25.0 & 19.2 & 14.0 & 11.7 & 10.7 & 12.1 & 15.5 & 18.8 & 22.5 & 25.3 & 28.1 & 25.4 & 17.6 & 12.4 & 7.7 & 6.1 \\
\hline 6.3 & 8.4 & 9.4 & 10.5 & 10.5 & 10.8 & 11.5 & 11.9 & 13.0 & 14.3 & 16.6 & 19.5 & 22.4 & 24.8 & 26.2 & 25.3 & 19.4 & 14.5 & 11.2 & 10.6 & 12.3 & 15.6 & 18.6 & 23.1 & 26.2 & 28.9 & 26.0 & 17.4 & \begin{tabular}{|l}
11.5 \\
\end{tabular} & 7.3 & 6.0 \\
\hline 6.3 & 8.1 & 9.1 & 10.1 & 10.4 & 10.5 & 11.1 & 12.1 & 13.2 & 14.8 & 17.1 & 19.7 & 21.9 & 24.8 & 25.4 & 25.0 & 19.7 & 14.9 & 11.5 & 10.5 & 12.8 & 15.1 & 19.1 & 23.0 & 27.4 & 29.9 & 25.2 & 17.6 & 10.9 & 7.1 & 6.0 \\
\hline 6.3 & 7.7 & 8.8 & 9.8 & 10.4 & 10.2 & 10.8 & 11.9 & 13.2 & 14.8 & 17.5 & 19.7 & 22.7 & 24.2 & 24.8 & 25.2 & 19.8 & 14.9 & 11.4 & 10.8 & 12.1 & 15.0 & 18.8 & 24.1 & 28.7 & 30.2 & 24.8 & 17.4 & 10.9 & 6.7 & 6.0 \\
\hline 6.3 & 7.6 & 8.4 & 9.8 & 10.1 & 10.0 & 10.8 & 11.8 & 13.0 & 15.2 & 17.6 & 19.9 & 23.2 & 23.6 & 24.4 & 25.0 & 19.9 & 14.7 & 11.7 & 11.1 & 12.2 & 14.7 & 18.4 & 25.5 & 29.8 & 29.9 & 23.9 & 18.3 & 10.6 & 6.6 & 6.0 \\
\hline 6.3 & 7.6 & 8.3 & 9.7 & 10.0 & 10.0 & 10.6 & 11.7 & 13.1 & 15.2 & 17.1 & 20.4 & 23.4 & 23.3 & 24.0 & 25.3 & 19.4 & 14.5 & 12.3 & 11.4 & 11.9 & 14.4 & 18.8 & 25.9 & 30.8 & 29.6 & 23.5 & 18.4 & \begin{tabular}{|l|}
10.5 \\
\end{tabular} & 6.6 & 6.0 \\
\hline 6.3 & 7.5 & 7.8 & 9.4 & 10.0 & 9.7 & 10.2 & 11.3 & 13.0 & 15.0 & 16.9 & 21.4 & 24.8 & 22.8 & 22.7 & 24.9 & 19.3 & 14.5 & 12.5 & 11.1 & 11.6 & 13.7 & 19.3 & 27.1 & 32.2 & 28.5 & 23.2 & 18.4 & 11.3 & 7.4 & 6.1 \\
\hline 6.3 & 7.5 & 7.9 & 9.5 & 10.1 & 9.6 & 10.1 & 11.3 & 12.9 & 14.3 & 16.9 & 21.4 & 25.1 & 23.0 & 22.8 & 24.3 & 19.4 & 14.3 & 12.4 & 10.9 & 11.3 & 14.4 & 19.6 & 27.3 & 32.1 & 28.6 & 23.2 & 18.5 & 11.5 & 7.4 & 6.1 \\
\hline 6.3 & 7.5 & 8.0 & 9.3 & \begin{tabular}{|l}
10.3 \\
\end{tabular} & 9.8 & 9.8 & 10.9 & 12.4 & 13.8 & 17.1 & 21.6 & 25.2 & 23.6 & 22.6 & 23.4 & 19.3 & 14.4 & 12.0 & 10.7 & 11.1 & 15.1 & 20.0 & 27.2 & 32.0 & 29.0 & 23.1 & 17.9 & 12.8 & 7.7 & 6.1 \\
\hline 6.3 & 7.6 & 7.9 & 9.7 & 9.9 & 9.8 & 9.9 & \begin{tabular}{|l|l}
10.7 \\
\end{tabular} & 11.7 & 13.6 & 17.3 & 21.6 & 25.1 & 24.4 & 22.4 & 22.5 & 18.8 & 15.2 & 11.2 & 10.3 & 11.9 & 15.0 & 20.5 & 27.7 & 31.0 & 29.2 & 23.0 & 17.7 & \begin{tabular}{|l|}
13.4 \\
\end{tabular} & 8.6 & 6.1 \\
\hline 6.3 & 7.8 & 8.3 & 9.8 & 10.0 & 9.7 & 10.1 & 10.1 & 10.9 & 13.4 & 17.3 & 21.5 & 25.0 & 25.1 & 22.5 & 22.0 & 18.3 & 15.3 & 10.1 & 10.0 & 12.7 & 15.2 & 20.7 & 27.9 & 30.0 & 29.4 & 23.7 & 17.3 & 14.0 & 9.6 & 6.1 \\
\hline 6.5 & 7.7 & 8.9 & 9.4 & 9.7 & 10.1 & 10.1 & 9.4 & 10.7 & 13.2 & 17.5 & 21.3 & 25.0 & 25.1 & 22.8 & 21.5 & 18.2 & 14.7 & 9.7 & 10.5 & 12.6 & 15.9 & 20.7 & 27.9 & 29.2 & 29.1 & 23.8 & 17.6 & 14.1 & 10.9 & 6.3 \\
\hline 6.7 & 7.8 & 9.5 & 9.4 & 9.6 & 10.1 & 10.0 & 9.0 & 10.2 & 13.1 & 17.2 & 21.2 & 25.0 & 24.9 & 23.4 & 21.4 & 17.8 & 13.2 & 9.9 & 11.0 & 12.8 & 15.7 & 20.9 & 27.5 & 29.0 & 28.4 & 23.9 & 18.7 & 13.9 & 12.3 & 6.6 \\
\hline 6.8 & 8.5 & 9.1 & 9.1 & 9.9 & 10.3 & 9.3 & 9.2 & 9.8 & 13.0 & 16.8 & 21.5 & 24.6 & 24.3 & 23.8 & 21.5 & 17.8 & 11.2 & 11.0 & 10.6 & 13.1 & 16.0 & 21.3 & 26.6 & 28.6 & 28.0 & 23.9 & \begin{tabular}{|l|l}
19.3 \\
\end{tabular} & \begin{tabular}{|l|}
14.6 \\
\end{tabular} & 13.3 & 7.1 \\
\hline 7.1 & 9.4 & 9.3 & 8.8 & 9.9 & 10.4 & 8.5 & 9.6 & 9.8 & 12.8 & 16.9 & 21.5 & 24.1 & 23.9 & 24.0 & 21.3 & 17.2 & 9.9 & 12.4 & 11.2 & 12.5 & 15.3 & 21.8 & 26.1 & 28.3 & 27.5 & 23.5 & 19.6 & 15.5 & 13.6 & 8.6 \\
\hline 8.0 & 9.2 & 8.8 & 8.8 & 9.7 & 9.6 & 8.8 & 9.7 & 10.7 & 12.8 & 16.3 & 20.9 & 23.5 & 23.4 & 23.7 & 20.8 & 15.5 & 10.8 & 12.7 & 12.8 & 12.9 & 15.9 & 21.6 & 25.8 & 27.0 & 26.0 & 23.1 & 19.9 & 15.7 & 13.4 & 12.2 \\
\hline 9.7 & 9.5 & 8.7 & 9.4 & 10.0 & 8.4 & 9.2 & 9.2 & 11.0 & 12.3 & 15.5 & 20.6 & 22.8 & 23.5 & 24.0 & 20.8 & 13.3 & 12.6 & 11.5 & 12.5 & 12.7 & 14.6 & 20.6 & 26.2 & 26.5 & 26.7 & 23.4 & 20.3 & 16.9 & 14.3 & 13.2 \\
\hline
\end{tabular}

Figure 4.23 Reconstructed attenuation rate distribution in the physical model 
Sandstone

Intrusion-1
Sandstone

Intrusion-2

\begin{tabular}{|r|r|r|r|l|l|l|l|}
\hline & & & & & & \\
\hline $20.1 \%$ & $11.2 \%$ & $13.0 \%$ & $26.9 \%$ & & & & \\
\hline $18.2 \%$ & $10.4 \%$ & $6.4 \%$ & $17.7 \%$ & & & & \\
\hline $18.8 \%$ & $9.9 \%$ & $4.0 \%$ & $12.9 \%$ & & & & \\
\hline $17.3 \%$ & $6.3 \%$ & $0.6 \%$ & $9.8 \%$ & & & & \\
\hline $16.7 \%$ & $6.4 \%$ & $4.1 \%$ & $5.9 \%$ & & & & \\
\hline $14.6 \%$ & $2.9 \%$ & $7.1 \%$ & $3.6 \%$ & & & & \\
\hline $15.0 \%$ & $1.3 \%$ & $10.6 \%$ & $6.8 \%$ & & & & \\
\hline $10.6 \%$ & $6.4 \%$ & $11.9 \%$ & $8.1 \%$ & & & & \\
\hline $5.7 \%$ & $10.5 \%$ & $10.8 \%$ & $11.5 \%$ & & & & \\
\hline $4.2 \%$ & $13.9 \%$ & $9.7 \%$ & $12.9 \%$ & & & & \\
\hline $0.2 \%$ & $19.3 \%$ & $5.7 \%$ & $14.1 \%$ & & & & \\
\hline $1.2 \%$ & $18.9 \%$ & $5.9 \%$ & $14.2 \%$ & & & & \\
\hline $0.8 \%$ & $18.4 \%$ & $7.4 \%$ & $14.6 \%$ & & & & \\
\hline $2.7 \%$ & $14.7 \%$ & $8.2 \%$ & $14.8 \%$ & & & & \\
\hline $3.3 \%$ & $11.3 \%$ & $8.9 \%$ & $12.2 \%$ & & & & \\
\hline $3.4 \%$ & $8.1 \%$ & $7.9 \%$ & $11.9 \%$ & & & & \\
\hline $2.0 \%$ & $7.3 \%$ & $5.2 \%$ & $11.4 \%$ & & & & \\
\hline $1.5 \%$ & $5.8 \%$ & $3.7 \%$ & $11.5 \%$ & & & & \\
\hline $3.3 \%$ & $4.9 \%$ & $1.8 \%$ & $13.0 \%$ & & & & \\
\hline $4.6 \%$ & $0.0 \%$ & $3.8 \%$ & $14.3 \%$ & & & & \\
\hline & & & & & & & \\
\hline
\end{tabular}

Figure 4.24 Relative error of the reconstructed attenuation rate in the physical model 


\section{CHAPTER 5 SUMMARY AND CONCLUSIONS}

This research was aimed at developing a laboratory physical modeling method to study the Radio Imaging Method (RIM). Two physical models have been constructed. In the physical scale models, the coal seam, the floor and roof strata and the geological anomalies were simulated with properly chosen simulation materials. Based on the physical modeling simulation theory and laboratory test, different ratios of sand, gypsum, and cement are used to build the floor and roof strata in the scale models. A mixture of sand, coal powder and gypsum was used to simulate the coal seam. A modified radar instrumentation setup, two $900 \mathrm{MHz}$ ground penetrating radar (GPR) antennas were used as the RIM transmitter and receiver, respectively. MiniRIM attenuation rate tomogram software was developed and validated. Two scale models with different geological anomalies were designed and tested with reconnaissance test and RIM tomogram, and the results have been analyzed.

During this research, the following findings and conclusions were obtained.

- A proper mixture of sand, gypsum, cement, and salt can be used to build the floor and roof strata in the scale models. A proper mixture of sand, coal powder, and gypsum can be used to simulate the coal seam. In general, the electrical conductivity in the rock (e.g. $0.1 \mathrm{~S} / \mathrm{m}$ ) is greater than the electrical conductivity in the coal seam (e.g. $0.0001 \mathrm{~S} / \mathrm{m}$ ). EM wave energy propagation occurs in the seam because the higher conductivity sedimentary rocks surrounding the seam force the radio wave energy to flow in the coal seam between the sedimentary rocks. According to the test results, different mixtures of sand, gypsum, cement, and salt have a higher electrical 
conductivity (up to $1 \mathrm{~S} / \mathrm{m}$ at a water content of $10 \%$ ). While the different mixtures of sand, coal powder, and gypsum have a lower electric conductivity (as low as $0.01 \mathrm{~S} / \mathrm{m}$ at a water content of $10 \%)$. The testing results also show that the mixtures of sand, gypsum, cement, and salt have a higher dielectric constant (up to 9 at the water content of 10\%). While the different mixtures of sand, coal powder, and gypsum have a lower dielectric constant (as low as $5 \mathrm{~S} / \mathrm{m}$ at the water content of $10 \%$ ). These EM properties of the simulation materials are very important for selecting the proper mixture ratios to build the roof/floor, coal seam, and the geological anomalies.

- Both the electrical conductivity and the dielectric constant increase with the increasing water content. Salt is a useful material which is mixed in the roof/floor simulation materials to increase the electrical conductivity. The mixture of materials with or without salt have a large effect on the electrical conductivity at high water content, while it has less effect at low water content.

- When building the physical scale model, it is not necessary to meet all the scale factors. Of all the scale factors, those for the electrical conductivity and dielectric constant are most important, because they directly affect the EM wave propagation and attenuation rate in the coal seam and at the geological anomalies.

- Based on the designed scale factor ratio and EM properties test results, the material of $\mathrm{S}: \mathrm{Co}: \mathrm{C}=5: 5: 1$ with water content at $6 \%$ was selected to simulate the coal seam (dielectric constant $=3.2$ and the electrical conductivity $=0.006 \mathrm{~S} / \mathrm{m}$ ). The mixture materials of S:G:C:SA=7:0.5:0.5:1 with water content at $6.5 \%$ (dielectric constant $=6$ and electrical conductivity $=0.5 \mathrm{~S} / \mathrm{m}$ ) was selected to simulate the roof/floor and the geological anomalies. 
- A high frequency radar system with separated $900 \mathrm{MHz}$ transmitter antenna and receiver antenna was used to test the laboratory physical model. Since RIM testing needs a separated transmitter antenna and receiver antenna, an antenna with the receiver part disabled was used as the transmitter; and an antenna with the transmitter part disabled was used as the receiver. A breakout box was used to connect the control unit (SIR II) and the antennas. This modified radar system met the requirement of the laboratory physical model RIM tests.

- The developed MiniRIM attenuation rate tomogram software is capable of processing the laboratory physical model RIM testing data and reconstructing the attenuation rate tomogram. A forward modeling study was conducted to analyze and validate the software. Different types of artificial geological anomalies structures were analyzed. Most of the forward modeling results showed that the attenuation rate of the geological anomalies were well reconstructed. The geological anomaly lying vertically in the coal seam can be the best reconstructed tomogram, while the transverse geological anomalies produced the worst reconstruction tomogram. That is because the path length through the anomaly by the most diagonal ray is not much greater than the path length through the anomaly by a direct ray. So for this kind of orientation anomaly, it will be difficult to reconstruct the anomaly tomogram.

- It is important to conduct the both a calibration test and a reconnaissance test before an actual RIM test. The calibration test can obtain the standard attenuation rate in the homogeneous coal seam, and the C-Factor which is used to generate the input data $Y_{i}$ $\left(Y=V_{0}-V_{i}-20 \log \left(r_{i}\right)\right)$. The reconnaissance test is an initial test which can be 
used to quickly and primarily detect the geological anomalies in the coal seam. The reconnaissance test results will be an important reference for the full RIM testing.

- Two physical models were constructed, but only one was suitable for reconstructing the attenuation rate tomogram. The first physical model was not very successful, because the first reason is because no salt was added in the material used to build the roof/floor, the difference in electrical conductivity between the roof/floor and the coal seam was insufficient; consequently, the attenuation rate in the coal seam and the roof/floor was very close. Another important reason was that the water content was not controlled in the physical model. When the water content was very small, the difference in electrical conductivity and dielectric constant was very small too. Because of these problems, it was difficult to detect changes in signal strength in different coal seam thickness.

- The second physical model was successful. The properly simulation materials were used to construct the coal seam, roof/floor, and the geological anomalies were selected based on the EM scale factors and the laboratory test results. The calibration test and reconnaissance test were conducted. The reconstructed attenuation rate tomogram closely matched the shape and location of the embedded geological anomalies in the coal seam except the $1 \mathrm{in}$. gap. The reconstructed attenuation rate of the geological anomalies in the coal seam had small relative errors. The results further testified that the simulation materials used in the physical model were correct, and that the RIM attenuation rate tomogram software is capable of processing the RIM testing data, and reconstructing the tomogram. 


\section{CHAPTER 6 RECOMMENDATIONS}

Based on the finding of the present study, the following recommendations are made:

- A database of the EM properties (dielectric constant and electrical conductivity) of mixture materials with different material ratios and water content needs to be established. This will help for the future physical model construction.

- Scale factors used in the physical model need to be further analyzed. The relationship of dimension scale factor and frequency scale factor is not considered in this study. So, the attenuation rate obtained in the physical model cannot be directly converted to the attenuation rate in the real condition. A new scale factor for the attenuation rate needs to be established.

- More physical models with different geological anomalies need to be built to further verify the MiniRIM attenuation rate tomogram software. 


\section{REFERENCES}

1. Agilent, Application Note 1217-1, Basics of Measuring the Dielectric Properties of Materials, http://www3.imperial.ac.uk/pls/portallive/docs/1/11949698.pdf.

2. Agilent, $85070 \mathrm{E}$ Dielectric Probe Kit $200 \mathrm{MHz}$ to $50 \mathrm{GHz}$, Technical Overview, http://cp.literature.agilent.com/litweb/pdf/5989-0222EN.pdf

3. Anggoro, B., N.I. Sinisuka, and P.M. Pakpahan, 2006, "Resistivity and dielectric constant characteristic of soil if are treated by water, salt and carbon", Properties and applications of Dielectric Materials, 8th International Conference on, 893-896.

4. Bates, R.G., W.J. Hamer, G.G. Manov and S.F. Acree, 1942, J. Res. Natl. Bur. Stand. 29, 183pp.

5. Bates, R.G., 1981, “The modern meaning of pH. CRC Crit.," Rev. Anal. Chem. 12, 247pp.

6. Becht, A., J. Tronicke, E. Appel., P. Dietrich, 2004, "Inversion strategy in crosshole radar tomogram using information of data subsets", Geophysics, Vol. 69, No. 1; 222-230.

7. Bernabini, M., E. Pettinelli, N. Pierdicca, S. Piro and L. Versino, 1995, "Field experiments for characterization of GPR antenna and pulse propagation", Journal of Applied Geophysics, vol.33, 63-76.

8. Daniels, D.J., 1996, Surface-penetrating radar--IEE Radar, Sonar, Navigation and Avionics, Series 6: London, The Institute of Electrical Engineers, 320 pp.

9. Davis, J.L. and A.P. Annan, 1989, "Ground-penetrating radar for high-resolution mapping of soil and rock stratigraphy”, Geophysical Prospecting, Vol. 37, 531-551.

10. Dines, K. A. and R.J. Lytle, 1979, “Computerized geophysical tomogram”, Proc. of the IEEE Vol. 67, 1065-1073.

11. Duncan, J., "General Discussion - Principles of Radio Imaging Method (RIM), RIM Survey Methods in the Detection and Delineation of Abandoned Mine Entries and Pillars", http://www.stolarhorizon.com.

12. Durst, R.A., 1975, "Standard Reference Materials: Standardization of $\mathrm{pH}$ Measurements", NBS Spec Publ. 260-53, National Bur. Standards, 48 pp.

13. Gilbert, P., 1972, "Iterative methods for the three-dimensional reconstruction of an object from projections", J. Theor. Biol. 36, 105-117.

14. Girouxa, B., E. Gloaguenb and M. Chouteau, 2007, "Bh_tomo-a Matlab borehole georadar 2D tomogram package", Computers \& Geosciences 33, 126-137. 
15. Goldstein, S.E., M.D Knoll, W. Barrash, and W.P. Clement, 2003, "Borehole radar attenuation tomogram during the Tracer/Time-Lapse Test at the Boise Hydrogeophysical Research Site", Proceedings of SAGEEP03, The Symposium on the Application of Geophysics to Engineering and Environmental Problems, April 6-10, San Antonio, TX, 147-162.

16. Gordon, R., 1974, "A tutorial on ART (algebraic reconstruction techniques)", IEEE Trans. Nucl. Sci. NS-21: 78-93.

17. Gordon, R., R. Bender, and G.T. Herman, 1970, "Algebraic reconstruction techniques (ART) for three-dimensional electron microscopy and X-ray photography", J. Theoret. Biol. 29, 471-481.

18. Hill, D., 1984, "Radio Radio Propagation in a coal seam and the inverse problem", Journal of Research, National Bureau of Standards, Vol. 89, No. 5, Sep.-Oct., 385-394.

19. Hyun, S. Y., Y.S. Jo, H.C. Oh, S.Y. Kim, and Y.S. Kim, 2007, "The laboratory scaled-down model of a ground-penetrating radar for leak detection of water pipes", Meas. Sci. Technol. $18,2791-2799$.

20. Johnson W.J., 2003, "Applications of the electrical resistivity method for detection of underground mine workings", Geophysical Technologies for Detecting Underground Coal Mine Voids, Lexington, KY, July, 28-30.

21. Fornies-Marquina, J.M., J.C., Martin, J. P. Martinez, J. L. Miranda, and C. Romero, 2003, "Dielectric characterization of coals", Canadian J. of Phys., 81, 599-610.

22. Kak, A.C. and M. Slaney, 1999, "Principles of Computerized Tomographic imaging", IEEE Press, The Institute of Electrical and Electronics Engineers, Inc., New York

23. King, R.W.P. and G. S. Smith, 1981, Antennas in Matter: Fundamentals, Theory, and Applications. Cambridge, MA: MIT Press, Chap. 12.

24. King, R.J., 1982, "Physical modeling of EM wave propagation over the earth", Radio Sci., Vol. 17, 103-116.

25. Kuhl, D.E. and R.Q. Edwards, 1963, "Image separation radioisotope scanning”, Radiology, 80, 653-662.

26. Lin, Y.M., 1984, Simulation Research on Rock Mechanics Experiment. Beijing: China Coal Industry Publishing House (in Chinese).

27. Newman, G.A., 1995, "Crosswell Electromagnetic Inversion Using Integral and differential Equations", Geophysics, Vol. 60, 899-911.

28. Kaczmarz, S., 1937, “Angenaherte auflosung von systemen linearer gleichungen”, Bull. Acad. Pol. Sci. Lett. A, Vol. 6-8A, 355-357. 
29. Knight, R. and A. Endres, 1990, "A new concept in modeling the dielectric response of sandstones: defining a wetted rock and bulk water system”, Geophysics 55, No. 5, 586-594.

30. Knoll, M.D., 1996, “A petrophysical basis for ground-penetrating radar and very early time electromagnetics, electrical properties of sand-clay mixtures", unpublished Ph.D. dissertation, University of British Columbia, $316 \mathrm{pp}$.

31. Lager, D.L. and R.J. Lytle, 1977, "Determining a subsurface electromagnetic profile from highfrequency measurements by applying reconstruction technique algorithms”, Radio Sci. 12, 249-260.

32. Liu L, J. Lane and Y. Quan, 1998, "Radar attenuation tomogram using the centroid frequency downshift method", J. Appl. Geophys, 40, 105-116.

33. Martinez, A. and A.P. Byrnes, 2001, "Modeling Dielectric-constant values of Geologic Materials: An Aid to Ground-Penetrating Radar Data Collection and Interpretation", Current Res. Earth Sci., Kansas Geol. Surv., pt. 1, vol. Bull. 247, 1-15.

34. Newman, G.A., 1995, “Crosswell Electromagnetic Inversion Using Integral and differential Equations", Geophysics, Vol.60, 899-911.

35. Monaghan, W.D., 2007, Experimental studies of electromagnetic signals to enhance Radio Imaging Meghod (RIM), MS. Thesis, West Virginia University, Morgantown, WV.

36. Nahin P.J., Oliver Heaviside: Sage in Solitude, 1988, The Life, Work and Times of An Electrical Genius of the Victorian Age, IEEE Press, New York.

37. Olhoeft, G.R., 1976, "Electrical properties of rocks", Physics and Chemistry of Rocks and Minerals, Strens, R. J. G., ed.: Wiley, London, 262-278.

38. Paige, C.C. and M.A. Saunders, 1982, "LSQR: An algorithm for sparse linear equations and sparse least squares", TOMS 8(1), 43-71.

39. Parkhomenko, E.I., 1967, Electrical Properties of Rocks, Plenum, New York.

40. Peden, I. C and J. Brew, 1995, “A laboratory scale model for the study of subsurface scattering in low-loss media with applications to ground penetrating radar", Journal of Applied Geophysics 33 109-118.

41. Peden, I. C. and J. Brew, 1995, “A laboratory scale model for the study of subsurface scattering in low-loss media with applications to ground penetrating radar", Journal of Applied Geophysics, Vol. 33, 109-118.

42. Peng, S. S., 2006, Longwall Mining, second edition, Morgantown, WV, 621 pp.

43. Peng, S. S., 2008, Coal Mine Ground Control, third edition, Morgantown, WV, 750 pp. 
44. Peng, S.S., H.C. Li, and Y.M. Jiang, 1987, Study of overburden movement due to shallow longwalling using large scale simulation model, West Virginia University, Morgantown, WV, $53 \mathrm{pp}$.

45. Qian, M.G. and T.C. Liu, 1996, Ground pressure and strata control. Beijing: China Coal Industry Publishing House (in Chinese), 335 pp.

46. Radcliff, R.D. and C.A. Balonis, 1979, "Reconstruction algorithms for geophysical applications in noisy environments". Proc. IEEE 67: 1060-1064.

47. Church, R.H., W.E. Webb, and J.R. Boyle, 1985, Ground penetrating radar for strata control, U.S. Bureau of Mines RI 8954. 15pp.

48. Shope, S.M., 1987, Electromagnetic Coal Seam Tomogram, Ph.D. dissertation, Pennsylvania State University, $292 \mathrm{pp}$.

49. Sinclair, G., 1948, "Theory of models of electromagnetic system", Proc. IEEE, 36 (11), 13641370.

50. Smith, G.S. and W.R. Jr. Scott, 1989, “A scale model for studying ground penetrating radars”, IEEE Trans. Geosci. Remote Sensing 27 358-363.

51. Smith, G.S. and W.R. Jr. Scott, 1990, "The use of emulsion to represent dielectric materials in electromagnetic scale models", IEEE Transactions on antennas nad propagation, Vol. 38, 323-334.

52. Stolarczyk, L.G. and R.C. Fry, 1990, "Radio Imaging Method (RIM) or Diagnostic Imaging of Anomalous geologic Structures in Coal Seam Waveguides", Transactions of Society for Mining, Metallurgy and Exploration, Inc., Vol. 288, 1806-1814.

53. Stolarczyk, L.G. and S.S. Peng, 2003, “Advanced Electromagnetic Wave Technologies for the Detection of Abandoned Mine Entries and Delineation of Barrier Pillars", Proc. Interactive Forum on Geophysical Technologies for Detecting Underground Coal Mine Voids, July, Lexington, $\mathrm{KY}$.

54. Stolarczyk, L.G., S.S. Peng and Y. Luo, 2003, "Imaging Ahead of Mining with RIM-IV Instrumentation and 3-D Tomogram Software", Proceedings of the 22nd International Conference on Ground Control in Mining, West Virginia University, Morgantown, WV, Aug. 136-143.

55. Stolarczyk, L.G. and G.L. Stolarczyk, 1999, "Electromagnetic seam wave mapping of roof rock condition across a longwall panel", Proceedings of 18th International Conference on Ground Control in Mining, West Virginia University, Morgantown, WV, 50-53. 
56. Stolarczyk, L.G., Y. Luo and S.S. Peng, 2004, "Demonstration of the Advanced Radio Imaging Method (RIM): Ground Truth Results for Three- Dimensional Imaging of Coal Seams", SME Annual Meeting, Denver, Co.

57. Stolarczyk, G.L., J. Duncan, I. Bausov, S.S. Peng, Y. Luo and M. Mosser, 2006, “Advanced Development of the Radio Imaging Method", Proceedings of 25th International Conference on Ground Control in Mining, West Virginia University, Morgantown, WV, 86-92.

58. Stolarczyk, G. L., H. Tehrani, B. Bancroft, G. Hasenfus, and S.S. Peng, 2004, "Development and Field Tests of Look-Ahead Radar and Horizon Sensing for Coal Cutting Drums", Proceedings of 25th International Conference on Ground Control in Mining, West Virginia University, Morgantown, WV, Aug., 93-98.

59. Stolar Horizon, Inc., 2004, Radio Imaging Method Tomogram Survey Report West Ridge Panel No. 5 Footage $5+00$ to $47+50$.

60. Tanabe, K., 1971, "Projection method for solving a singular system", Numer. Math., Vol. 17, 203-214.

61. Thomson, S., 1993, "Bringing Geophysics into the Mine: Radio Attenuation Imaging and Mine Geology”, Exploration Geophysics, 24, 805-810.

62. Wait, J.R. (ed.), 1971, Electromagnetic Probing in Geophysics, Boulder, Colorado: The Golem Press.

63. Wait, J.R., 1982, Geo-Electromagnetism. New York, New York: Academic Press, a Subsidiary of Harcourt Brace Jovanovich, Publishers.

64. Wait, J.R. and Hill, D. A., 1976, “Analytical Investigation of Electromagnetic fields in Mine Environments (Contract H0155088, NOAA, US Dept. Commerce)", BuMines OFR 53-77, Nov. 15, $200 \mathrm{pp}$.

65. Wanstedt, S., S. Carlsten and S. Tire'n, 2000, "Borehole radar measurements aid structure geological interpretations", Journal of Applied Geophysics, 43. 227-237.

66. $\mathrm{Wu}, \mathrm{S} ., 1988$, Electromagnetic wave propagations in disrupted coal seam, MS Thesis, Pennsylvania State University, 872 pp.

67. Young, J. and G. Rogers, 1994, “Australian Development of Tomographic Radio Imaging as a New Tool in Mining Geophysics”, BUTSURI-TANSA, Vol.47, No.4, 249-255.

68. Zhao, H., 2005, Application of Radio Imaging Method (RIM-IV) in Detection Geological Anomalies-Two Case Study, MS. Thesis, West Virginia University, Morgantown, WV. 


\section{APPENDIX PHYSICAL MODELS CONSTRUCTION AND TESTING}

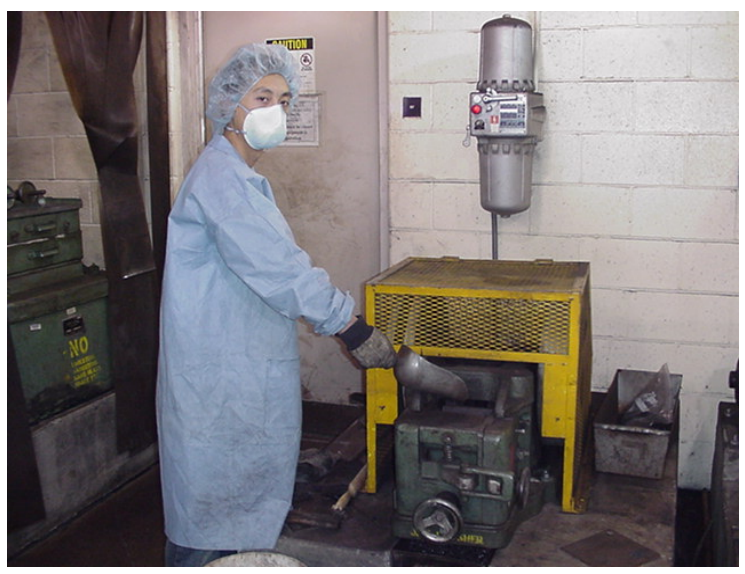

Appendix 1 Jaw crusher for coal powder preparation

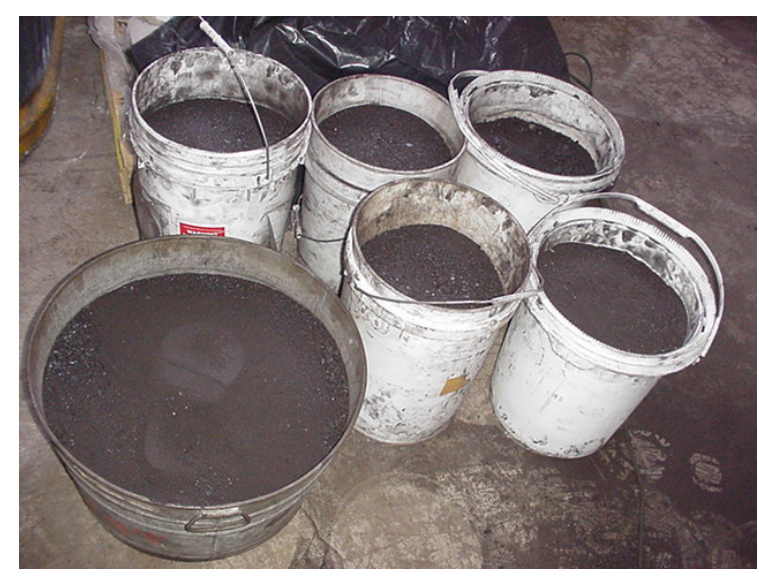

Appendix 3 Coal powder

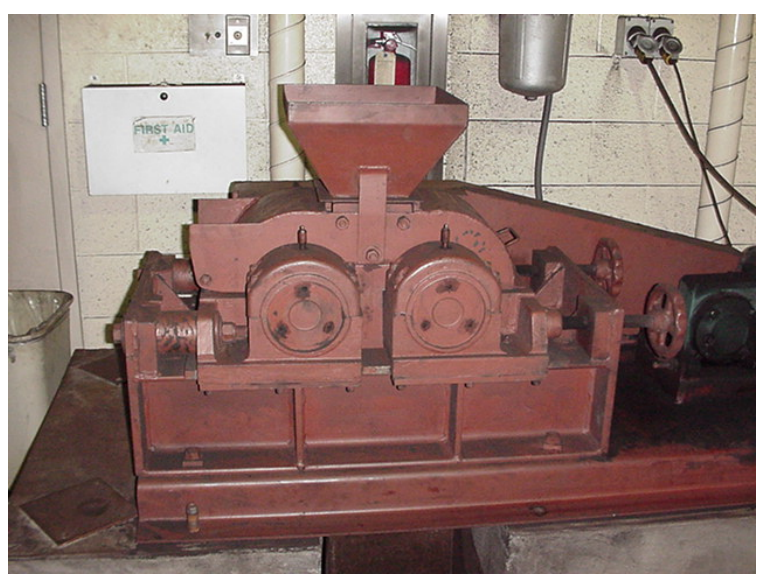

Appendix 2 Double roll crusher for coal powder preparation

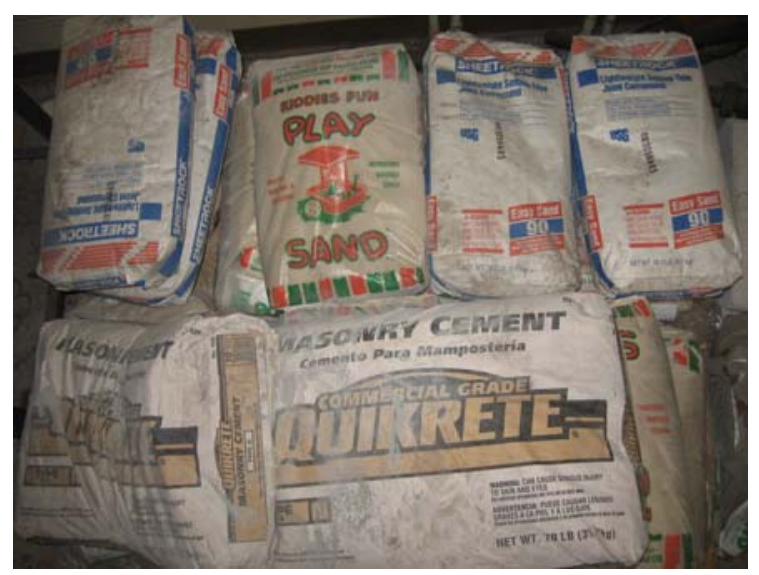

Appendix 4 Sand, cement and gypsum 


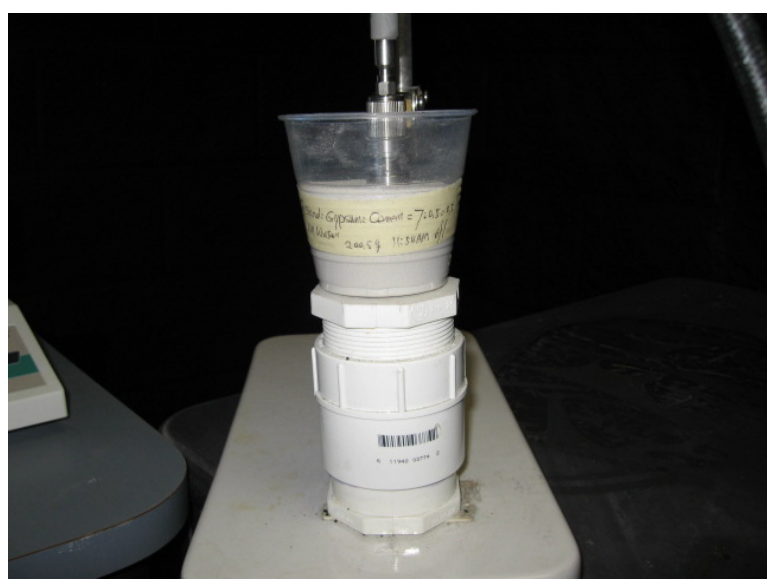

Appendix 5 Sample testing-1

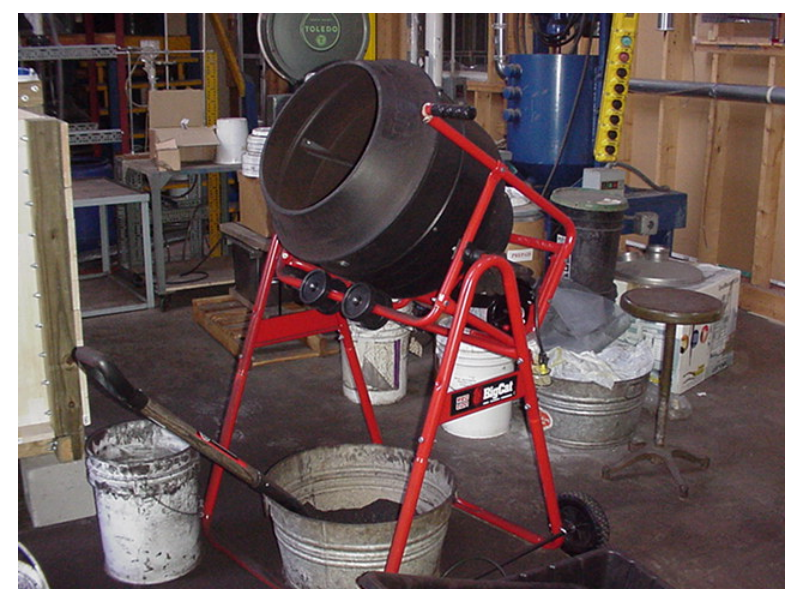

Appendix 7 Material mixture equipment

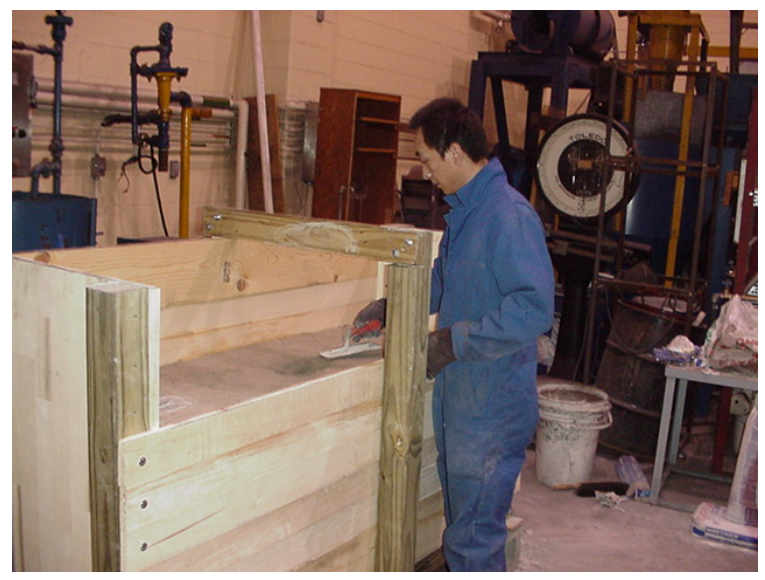

Appendix 9 Model construction-1

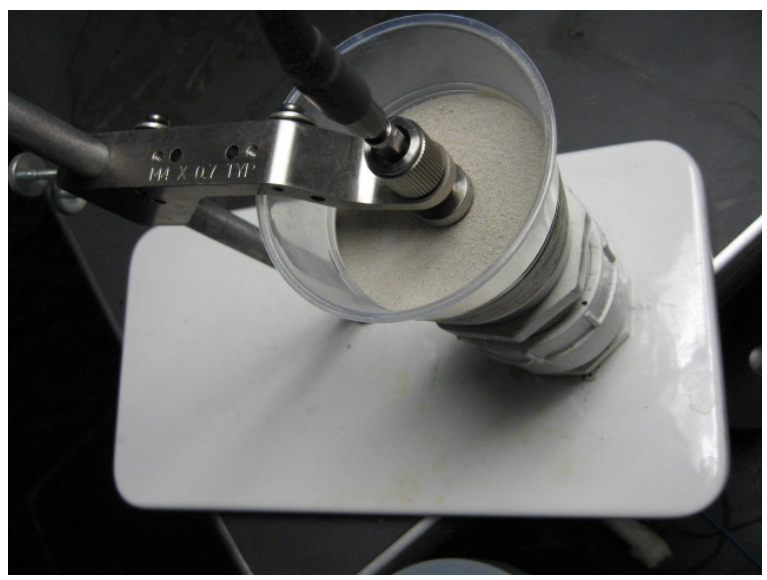

Appendix 6 Sample testing-2

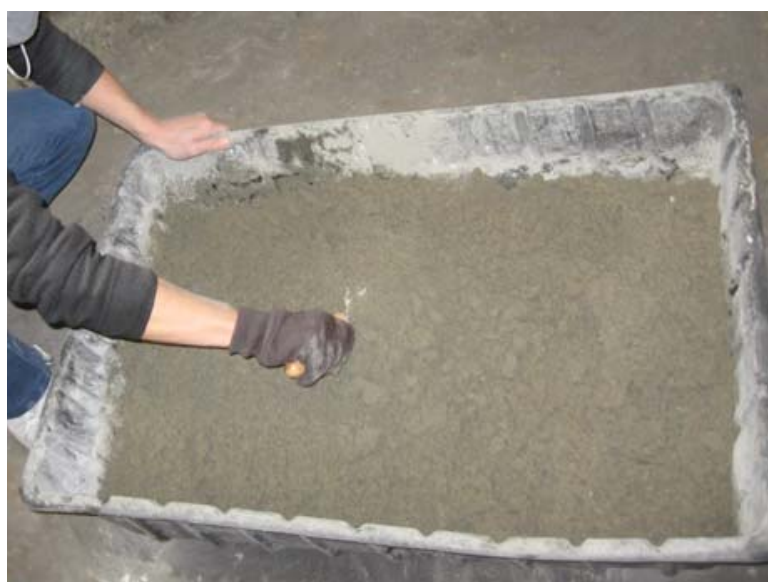

Appendix 8 Materials mixture

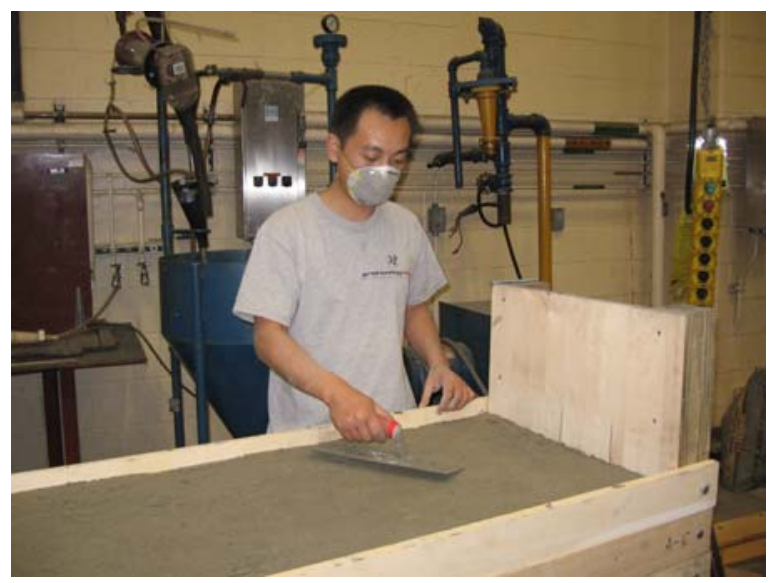

Appendix 10 Model construction-2 


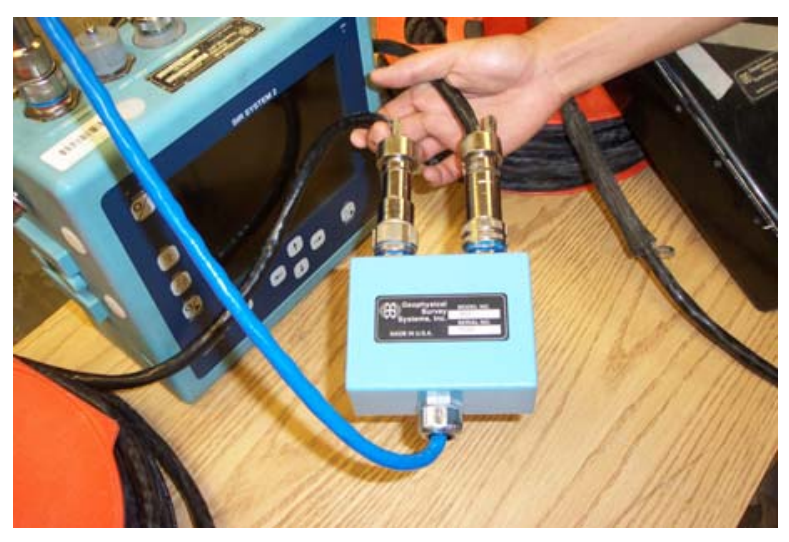

Appendix 11 Testing equipment

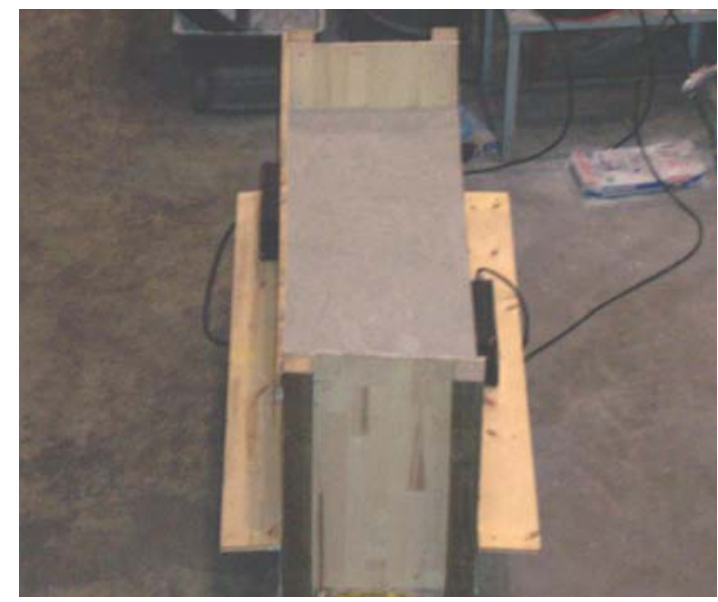

Appendix 13 Model testing-1

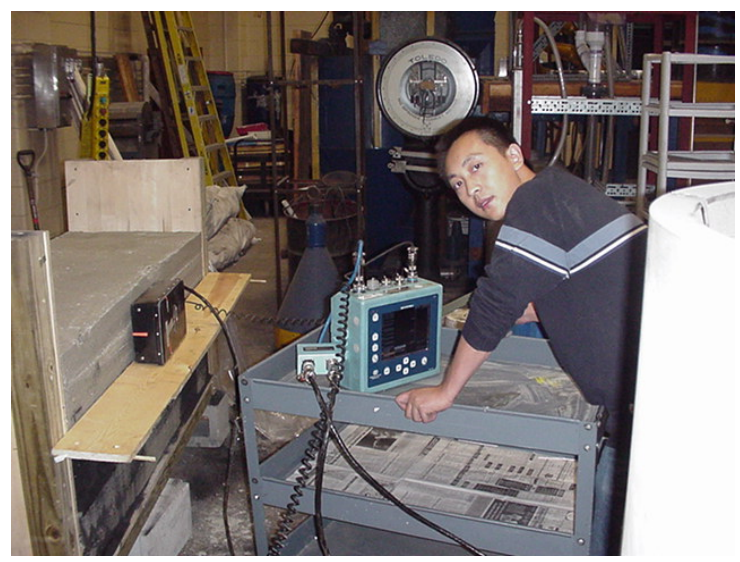

Appendix 15 Model testing-3

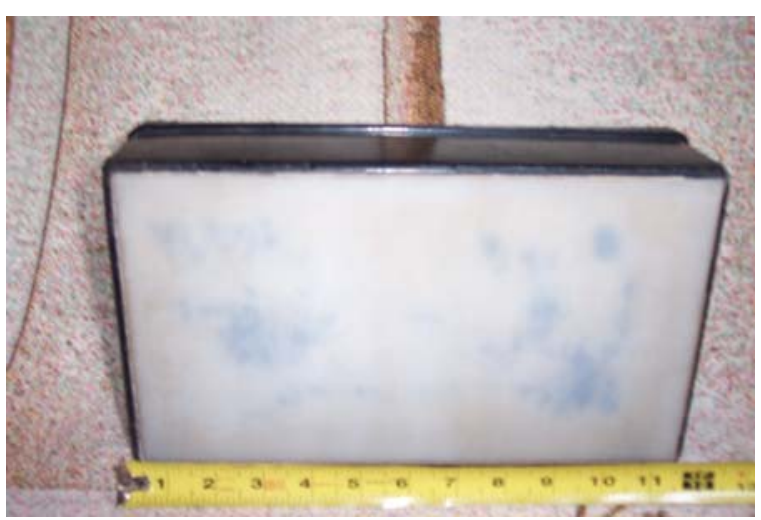

Appendix $12900 \mathrm{MHz}$ antenna

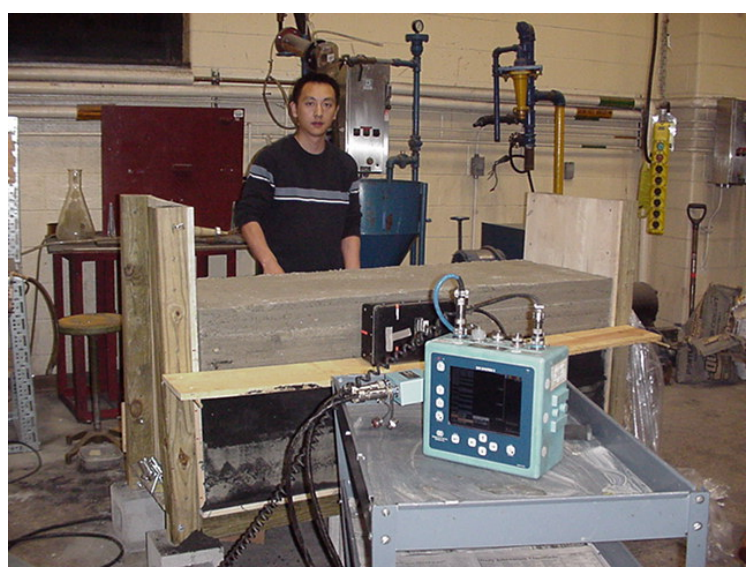

Appendix 14 Model testing-2

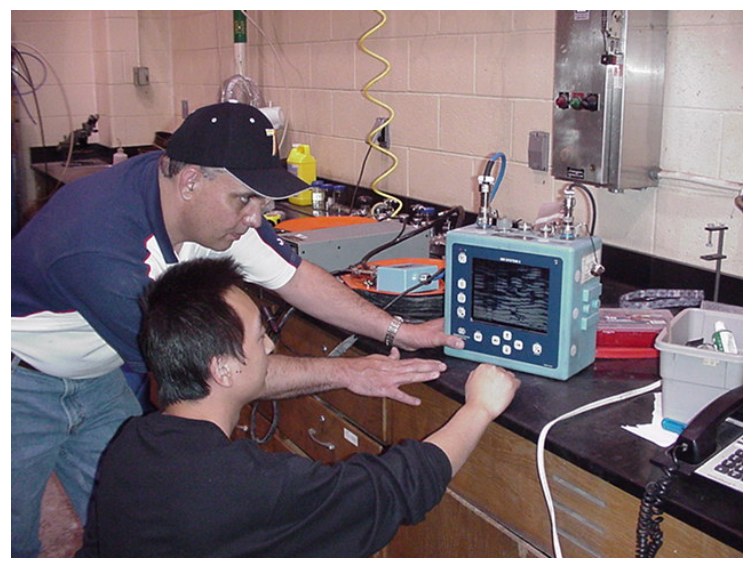

Appendix 16 Data analysis 